\title{
Extraction of Uranium from Seawater: Design and Testing of a Symbiotic System
}

by

\author{
Maha Niametullah Haji
}

B.S., B.A., University of California, Berkeley (2012)

S.M., Massachusetts Institute of Technology (2015)

Submitted in partial fulfillment of the requirements for the degree of

Doctor of Philosophy

at the

MASSACHUSETTS INSTITUTE OF TECHNOLOGY

and the

WOODS HOLE OCEANOGRAPHIC INSTITUTION

June 2017

(c) 2017 Maha N. Haji. All rights reserved.

The author hereby grants to MIT and WHOI permission to reproduce and to distribute publicly paper and electronic copies of this thesis document in whole or in part in any medium now known or hereafter created.

Author

Departments of Mechanical Engineering, MIT \& Applied Ocean Science and Engineering, WHOI May 26, 2017

Certified by .

Alexander H. Slocum Pappalardo Professor of Mechanical Engineering, MIT Thesis Supervisor

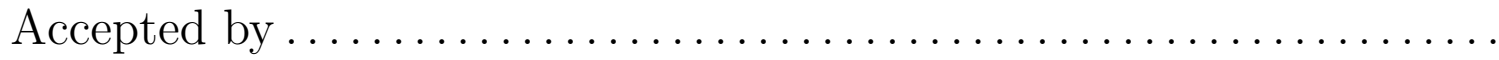

Rohan Abeyaratne

Chair, Department Committee on Graduate Studies, MIT

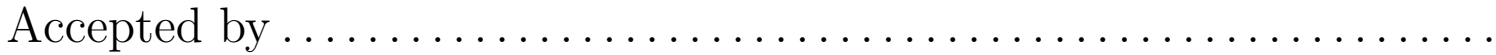

Henrik Schmidt

Chair, Joint Committee for Applied Ocean Science and Engineering, MIT 


\title{
Extraction of Uranium from Seawater: Design and Testing of a Symbiotic System
}

by

\author{
Maha Niametullah Haji
}

\author{
Submitted to the Departments of Mechanical Engineering, MIT \\ \& Applied Ocean Science and Engineering, WHOI \\ on May 26, 2017, in partial fulfillment of the \\ requirements for the degree of \\ Doctor of Philosophy
}

\begin{abstract}
Seawater is estimated to contain 4.5 billion tonnes of uranium, approximately 1000 times that available in conventional terrestrial resources. Finding a sustainable way to harvest uranium from seawater will provide a source of nuclear fuel for generations to come, while also giving all countries with ocean access a stable supply. This will also eliminate the need to store spent fuel for potential future reprocessing, thereby addressing nuclear proliferation issues as well. While extraction of uranium from seawater has been researched for decades, no economical, robust, ocean-deployable method of uranium collection has been presented to date. This thesis presents a symbiotic approach to ocean harvesting of uranium where a common structure supports a wind turbine and a device to harvest uranium from seawater. The Symbiotic Machine for Ocean uRanium Extraction (SMORE) created and tested decouples the function of absorbing uranium from the function of deploying the absorbent which enables a more efficient absorbent to be developed by chemists.

The initial SMORE concept involves an adsorbent device that is cycled through the seawater beneath the turbine and through an elution plant located on a platform above the sea surface. This design allows for more frequent harvesting, reduced downtime, and a reduction in the recovery costs of the adsorbent. Specifically, the design decouples the mechanical and chemical requirements of the device through a hard, permeable outer shell containing uranium adsorbing fibers. This system is designed to be used with the 5-MW NREL OC3-Hywind floating spar wind turbine.

To optimize the decoupling of the chemical and mechanical requirements using the shell enclosures for the uranium adsorbing fibers, an initial design analysis of the enclosures is presented. Moreover, a flume experiment using filtered, temperaturecontrolled seawater was developed to determine the effect that the shells have on the uptake of the uranium by the fibers they enclose. For this experiment, the AI8 amidoxime-based adsorbent fiber developed by Oak Ridge National Laboratory was used, which is a hollow-gear-shaped, high surface area polyethylene fiber prepared by radiation-induced graft polymerization of the amidoxime ligand and a vinylphosphonic acid comonomer.

The results of the flume experiment were then used to inform the design and fabrication of two 1/10th physical scale SMORE prototypes for ocean testing. The
\end{abstract}


AI8 adsorbent fibers were tested in two shell designs on both a stationary and a moving system during a nine-week ocean trial, with the latter allowing the effect of additional water flow on the adsorbents uranium uptake to be investigated. A novel method using the measurement of radium extracted onto $\mathrm{MnO}_{2}$ impregnated acrylic fibers to quantify the volume of water passing through the shells of the two systems was utilized.

The effect of a full-scale uranium harvesting system on the hydrodynamics of an offshore wind turbine were then investigated using a 1/150th Froude scale wave tank test. These experiments compared the measured excitation forces and responses of two versions of SMORE to those of an unmodified floating wind turbine.

With insights from the experiments on what a final full-scale design might look like, a cost-analysis was performed to determine the overall uranium production cost from a SMORE device. In this analysis, the capital, operating, and decommissioning costs were calculated and summed using discounted cash flow techniques similar to those used in previous economic models of the uranium adsorbent.

Major contributions of this thesis include fundamental design tools for the development and evaluation of symbiotic systems to harvest uranium or other minerals from seawater. These tools will allow others to design offshore uranium harvesting systems based on the adsorbent properties and the scale of the intended installation. These flexible tools can be tuned for a particular adsorbent, location, and installation size, thereby allowing this technology to spread broadly.

Thesis Supervisor: Alexander H. Slocum

Title: Pappalardo Professor of Mechanical Engineering, MIT 


\section{Acknowledgments}

First and foremost, I want to sincerely thank my $\mathrm{PhD}$ advisor, Prof. Alexander H. Slocum, for his support and guidance throughout this project. Thank you for always being excited about my research challenges (a.k.a. opportunities) and being a generally enthusiastic presence in my graduate career. Moreover, thank you for being a kind and caring advisor, for always looking out for your students, and ensuring we keep physically active.

Secondly, I would like to thank my thesis committee, Prof. Paul Sclavounos, Prof. Michael Driscoll and Dr. Ken Buesseler, for their insightful comments, and suggestions that helped guide this research. I would also like to thank Dr. Ken Buesseler for the collaborations that led to much of the experimental validation of my research work, described in chapters $\mathbf{0}$ and $\mathbf{6}$.

I would like to thank Prof. Martin Culpepper for his mentorship. Thank you for your advice and guidance over the past year. I sincerely appreciate you taking the time to help improve the outcome of my graduate career.

Thank you to my fellow Precision Engineering Research Group members, especially Jonathan Abbott, Megan O'Brien, Tom Cervantes, Amanda Hamlet, Nevan Hanumara, Hilary Johnson, Douglas Jonart, PK, Jocelyn Kluger, Shahd Labib, Kevin Simon, David Taylor, and Tyler Wortman. Your research advice, and discussions have helped me tremendously during my $\mathrm{PhD}$ and your friendship has made the lab a welcoming environment.

Thanks to Irina Gaziyeva, Leslie Regan, Joan Kravit, Ronni Schwartz, Kris Kipp, Leanora Fraser, and Julia Westwater for their unending help with all administrative issues.

Many thanks to Jessica Drysdale at Woods Hole Oceanographic Institute for all her help in preparing and analyzing the results from my lab and ocean experiments. Thanks to Dr. Chris Janke and his team at Oak Ridge National Laboratory for the preparation of the adsorbent fibers for use in this project, and to Dr. Gary Gill and his team at the Pacific Northwest National Laboratory for the analysis of the fibers post-deployment. Their assistance was invaluable in the work described in chapters 田 and 6 .

I would like to thank Dr. Erich Schneider and Maggie Flicker Byers at the University of Texas at Austin for their help in adapting their uranium from seawater cost analysis tool for use with a symbiotic deployment strategy, such as the one presented in my thesis. The work presented in chapter $\mathbb{\nabla}$ is a result of this collaboration.

Thanks to the staff and students of Massachusetts Maritime Academy for their help, support, and enthusiasm for allowing me to take over a part of their dock with my prototype for all of the summer and fall of 2016. Specifically, Tom Pham, Prof. Bill Hubbard, Bill Klimm, Thomas McEntee, Thanh Nguyen, Judson Poole, James Wall, Matthew Simms, Jimmy Benedetto, John Edwards and Walter Yohai played an integral role in ensuring the success of the study presented in chapter 6 .

Thank you to all the undergraduate students who have helped me throughout the years working on this project: Chukwunenye Anagbogu, Phoophie Chavez, Cyndia Cao, Justin Carrus, Cedric Delmy, Patrick Everett, Jorge Gonzalez, Jessie Hsiao, 
Jasmine Lennert, Bo Paulson, Costa Rivas, and Charles Vitry.

Thank you to the MIT MakerWorkshop for providing me with a home away from home, a place where folks are friendly and welcoming and eager to help me solve my design challenges.

Thank you to my friends who have been there for me and have made the past few years enjoyable: Jay Brett, Giulia DeSalvo, Lucy Du, Margaux Filippi, Julia Hopkins, Jacob Izraelavitz, Leah Mendelson, Katie Smyth, Marcel Thomas, and Sarah Thornton.

To my family: my mother, father, and brother. Thank you for your never-ending faith in my abilities, encouragement, and understanding throughout my life. I would not be the person I am today without you.

Last, but most certainly not least: Sasan. Thank you for your support and caring throughout the process. I could certainly not have done this without tour faith in me over the years and your help in every way possible. Thank you for always being there for me and for helping me to continue to grow each and every day.

This work was supported by the U.S. Department of Energy Office of Nuclear Energy under Contract No. DE-NE0008268 and by the National Academies Keck Futures Initiative.

This material is based upon work supported by the National Science Foundation Graduate Research Fellowship under Grant No. \#1122374. Any opinion, findings, and conclusions or recommendations expressed in this material are those of the author(s) and do not necessarily reflect the views of the National Science Foundation. 


\section{Contents}

$\begin{array}{ll}\text { List of Figures } & 11\end{array}$

$\begin{array}{ll}\text { List of Tables } & 21\end{array}$

1 Introduction 23

1. Motivation . . . . . . . . . . . . . . . . . . . . . 23

1.2 Terrestrial tranium . . . . . . . . . . . . . . . . . . 24

L.3 Seawater Uranium Extraction . . . . . . . . . . . . . . 26

1.4 Symbiotic Designs . . . . . . . . . . . . . . . . . . . . . . . . . 28

1.5 Feasibility . . . . . . . . . . . . . . . . . . . . . . . . 30

1.6 Thesis overview . . . . . . . . . . . . . . . . . . . . . . . . . . 31

2 Adsorbent Characteristics $\quad 33$

2.1 Adsorbent overview and properties . . . . . . . . . . . . . . . . . . . . . . 33

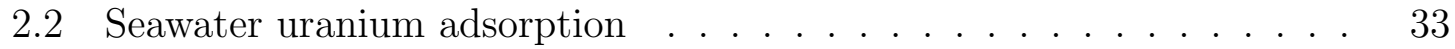

2.3 Elution and regeneration . . . . . . . . . . . . . . . . . . . . . . . . . . . . . . . . . . . . . . . . . 35

2.4 Adsorbent behavion . . . . . . . . . . . . . . . . . . . . . . . . . . . . . . . . . . . .

2.4 .1 Uptake . . . . . . . . . . . . . . . . . . . 35

2.4 .2 Adsorbent Degradation . . . . . . . . . . . . . . . . . . . . . . . . . . . . . 36

2.4 .3 Mechanical Parameters . . . . . . . . . . . . . . . . 37

2.4 .4 Sorption Optimization . . . . . . . . . . . . . . . . . . . . 38

2.5 Experimentally tested adsorbent uptake . . . . . . . . . . . . . . 40

2.5.] Adsorbent Performance Characteristics . . . . . . . . . . . . . 40

2.5 .2 Temperature Dependence . . . . . . . . . . . . . . . . . . 40

2.5.3 Biofouling Effects . . . . . . . . . . . . . . . . . . . . . 43

2.5 .4 Influence of Current Velocity . . . . . . . . . . . . . . . . . . . 45

2.5.5 Potential Adsorbent Loss . . . . . . . . . . . . . . . 46

2.6 Chapter Summary . . . . . . . . . . . . . . . . . . . . . . . . 47

3 Shell Enclosure Strategy 49

3.1 Motivation . . . . . . . . . . . . . . . . . . . . . 49

3.2 Theory . . . . . . . . . . . . . . . . . . . . . . . . . . . . . . . . 49

3.3 Adsorbent 1 nterion . . . . . . . . . . . . . . . . . . . 51

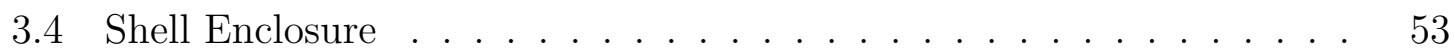

3.5 Conclusions . . . . . . . . . . . . . . . . . . 55 
$4 \quad$ Flume Testing of Shell Enclosures $\quad 59$

4.1 Method . . . . . . . . . . . . . . . . . . . . . . . . . 59

$4.1 .1 \quad$ Shell Enclosure Design and Fabrication . . . . . . . . . . . 59

4.1 .2 Design Analysis . . . . . . . . . . . . . . . . . . . . . . . . . . 63

4.1 .3 Adsorbent preparation, sampling, and analysis . . . . . . . . . 65

42 Results . . . . . . . . . . . . . . . . . . . . . . . . . . . . . . . . 65

4.3 Discussion $\ldots \ldots \ldots \ldots . \ldots \ldots$

$5 \quad$ Symbiotic Machine for Ocean uRanium Extraction (SMORE) $\quad 71$

5.1 Functional Requirements . . . . . . . . . . . . . . . . 71

5.2 Elution and Regeneration . . . . . . . . . . . . . . . . . . 72

5.3 Mooring and Recovery . . . . . . . . . . . . . . . . . . . 72

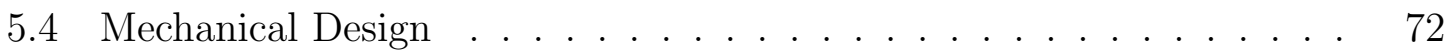

5.4 Ball-Chain Net . . . . . . . . . . . . . . . . . 73

5.4 .2 Roller design . . . . . . . . . . . . . . . . . . . . . . . 75

5.4 .3 Platform length . . . . . . . . . . . . . . . . . 76

5.4 .4 Elution and Regeneration . . . . . . . . . . . . 76

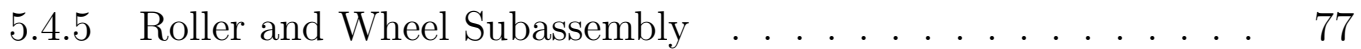

5.4 .6 Power Requirement . . . . . . . . . . . . . . . . . . . . 78

5.4 .7 Design Analysis . . . . . . . . . . . . . . . . . . . . . . . . . . 82

5.5 Chapter Summary $\ldots \ldots \ldots \ldots$

6 SMORE 1/10th Physical Scale Prototype Ocean Test 85

$6.1 \quad 1 / 10$ th physical scale prototypes $\ldots \ldots \ldots \ldots \ldots \ldots$

6.1 .1 System 1: Stationary net system . . . . . . . . . . . . . 85

6.1 .2 System 2: Continuous net system . . . . . . . . . . . 88

6.2 Ocean test site . . . . . . . . . . . . . . . . . . . . . 88

6.3 Shell enclosure net . . . . . . . . . . . . . . . . . . . . . . . . . . . . 89

6.4 Adsorbent deployment and sampling . . . . . . . . . . . . . . . . 91

6.5 Ocean test measurements . . . . . . . . . . . . . . . . . . . 92

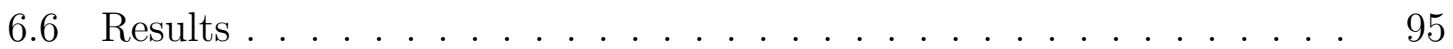

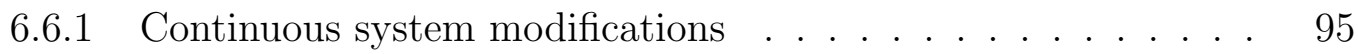

6.6 .2 Sensor data . . . . . . . . . . . . . . . . . . . . . . . 96 96

6.6 .3 Biofouling . . . . . . . . . . . . . . . . . . . . . . . . . . 98

6.6 .4 Water flow rate . . . . . . . . . . . . . . . . . . . . . 99

6.6 .5 Uranium uptake . . . . . . . . . . . . . . . . . . . . . 102

6.7 Discussion . . . . . . . . . . . . . . . . . . . . . . 105

7 SMORE Hydrodynamic Response 109

7.1 Theory . . . . . . . . . . . . . . . . . . . . . . . . . . . . . . . . 109

7.2 Experimental Setup . . . . . . . . . . . . . . . . . . . . . . . . 111

7.2 .1 Model scaling . . . . . . . . . . . . . . . . . . . . 111

7.2 .2 Models for testing . . . . . . . . . . . . . . . . . . . . . . . . . 112

7.2 .3 Experimental facilities . . . . . . . . . . . . . . . . . 114

72.4 Excitation Forces . . . . . . . . . . . . . . . . . . 114 
7.2 .5 Response Amplitude Operators . . . . . . . . . . . . . . . . . 115

7.3 Results . . . . . . . . . . . . . . . . . . . . . . . . . . . . . . . 118

7.3.] Excitation Forces . . . . . . . . . . . . . . . . . . 118

7.3 .2 Response Amplitude Operators . . . . . . . . . . . . . . . . . 120

7.4 Discussion . . . . . . . . . . . . . . . . . . . . . . . 122

8 SMORE Cost-Analysis $\quad \mathbf{1 2 5}$

8.1 Discounted Cash Flow Methodology . . . . . . . . . . . . . . . . . . . 125

8.2 Cost Components . . . . . . . . . . . . . . . . . . . . . . 127

8.2 .1 Adsorbent Production Cost . . . . . . . . . . . . . . . . . . . 127

8.2 .2 Mooring and Deployment Cost . . . . . . . . . . . . . . . . . 128

8.2 .3 Elution and Regeneration Cost . . . . . . . . . . . . . . . . . 129

8.3 Parameter Space . . . . . . . . . . . . . . . . . . . . . . 130

8.3 .1 Length of campaign . . . . . . . . . . . . . . . . . . . . . 130

8.3 .2 Temperature . . . . . . . . . . . . . . . . . . . . . . 130

8.3 .3 Degree of grafting . . . . . . . . . . . . . . . . . . . . . . . . . 130

8.3 .4 Number of reuses . . . . . . . . . . . . . . . . . . 131

8.3 .5 Degradation . . . . . . . . . . . . . . . . . . . . . . . 131

8.3 .6 Biofouling . . . . . . . . . . . . . . . . . . . . . . . . . 132

8.4 Reference Deployment Strategy . . . . . . . . . . . . . . . . . . . . . 132

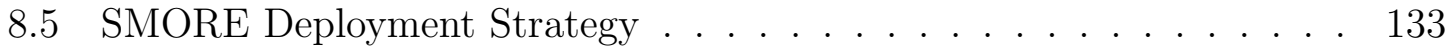

8.6 Results . . . . . . . . . . . . . . . . . . . . . . 134

8.7 Conclusion . . . . . . . . . . . . . . . . . . . . . 137

9 Seawater Uranium Suitability of Harvesting Index (SUSHI) 141

9.1 Motivation . . . . . . . . . . . . . . . . . . 141

9.2 Incorporated factors $\ldots \ldots \ldots \ldots$. . . . . . . . . . . . . . . 141

9.2.1 Wind Power . . . . . . . . . . . . . . . . . . . . . . 141

9.2 .2 Adsorbent capacity . . . . . . . . . . . . . . . . . . . . . . 143

9.3 Suitability Index . . . . . . . . . . . . . . . . . . . . . . . . . 143

9.4 Datasets . . . . . . . . . . . . . . . . . . . . . . . . . . . 143

9.4.1 Ocean surface winds . . . . . . . . . . . . . . . . . . . . 144

9.4 .2 Sea surface temperature . . . . . . . . . . . . . . . . . . . . 144

9.5 GUTPresentation . . . . . . . . . . . . . . . . . . . . . . . . 145

9.5.] GUTPresentation . . . . . . . . . . . . . . . . . 145

9.5 .2 Data output . . . . . . . . . . . . . . . . . . . . . . . . . . . . 149

9.5.3 Reminders: Dos and DoNTs . . . . . . . . . . . . . . 150

9.5.4 An example . . . . . . . . . . . . . . . . . . . . 151

9.6 Future Work . . . . . . . . . . . . . . . . . . . . . . . . . . . . . . 152

10 Conclusions and Future Work $\quad 155$

$\begin{array}{lr}\text { Bibliography } & 159\end{array}$ 


\section{List of Figures}

1-1 Global distribution of identified resources of uranium with a production cost of $<\$ 130 / \mathrm{kgU}$ among 15 countries that are either major uranium producers or have significant plans for growth of nuclear generating capacity. These 15 countries are endowed with $95 \%$ of the identified global resource base in this cost category - data as of January 1, 2015 (OECD Nuclear Energy Agency, 2016). Note that uranium with a production cost of $<\$ 50 / \mathrm{kgU}$ is considered the limit of "conventional" terrestrial resources . . . . . . . . . . . . . . . .

1-2 Uranium spot price from 1948-2015 in $2013 \$ / \mathrm{kgU}$. Adapted from Rothwell (2016). Uranium prices 1948-1972 from US Department of Energy (1981), converted to monthly prices by interpolation from midyear to mid-year, and converted to 2013 dollars using the Producer Price Index (Federal Reserve Bank of St. Louis, 2015). Prices January 1973-December 2006 are from Australian Bureau of Agricultural and Resource Economics and Sciences. Prices since 2006 were collected quarterly from the $\mathrm{UxC}$ website and converted to approximate monthly prices (Ux Consulting Company, LLC, 2015). . . . . . . . . . . . . . 25

1-3 Uranium collection system for adsorbent stacks (Seko et al., 2003). . . 27

1-4 Uranium collection system for braid adsorbent (Tamada et al., 2006). 27

1-5 Three-dimensional view of continuous uranium recovery system with adsorbent belt looped around the turbine mast proposed by Picard et al. (2014). The elution plant is housed on the upper platform out of the seawater . . . . . . . . . . . . . . . . 29

1-6 1/50th scale model of Picard et al. (2014) being tested in a pool. . . . $\quad 29$

1-7 Cost analysis of WUSABI as initially designed by Picard et al. (2014), compared to the reference kelp deployment strategy (Byers et al., 2016). 30

2-1 Three possible bonding motifs between amidoxime and metal ions (indicated by the red M): (a) the metal ion is bound to the oxygen atom, (b) the metal ion is bound to a five-membered chelate ring with oxygen and nitrogen donor atoms, and (c) the metal ion is bound with the $\mathbb{N - O b o n d ~ o f ~ o x i m i d o ~ . ~ . ~ . ~ . ~ . ~ . ~ . ~ . ~ . ~ . ~ . ~ . ~ . ~ . ~ . ~ . ~ . ~ . ~ . ~ . ~}$

2-2 Adsorption kinetics. Resulting uranium concentration and uranium recovering (g/kg-ads) for a harvest period of (a) 60 days and (b) 30 days for the AF1 adsorbent. . . . . . . . . . . . . . . . 36 
2-3 Recovery rate of uranium, $R$, as a function of the harvest time, $T_{h}$, for the AFT adsorbent . . . . . . . . . . . . . . . . .

2-4 Amount of uranium adsorbed in g-U/kg-ads, $\Gamma_{n}$, as a function of harvest time, $t$, and number of elution cycles, $n$ considering the AF1 adsorbent with $\beta_{\max }=5.421 \mathrm{~kg}-\mathrm{U} / \mathrm{t}$-ads, $K_{D}=22.6$ days, and $d=5$ (Gill et al., 2016). The value resulting from $t$ and $n$ from the study conducted by Picard et al. (2014) is indicated by the red star. Parameter combinations outside of the one year timeframe are shaded. The optimal value within the limited region is shown by the red circle. . .

2-5 As in figure 2-4 but for the amount of adsorbent required in $\mathrm{kg} \mathrm{to}$ harvest $1.2 \mathrm{t}-\mathrm{U}$ annually. . . . . . . . . . . . . . . . . . . . .

2-6 Uranium adsorbent capacity (g-U/kg-ads) as a function of soaking time in days and water temperature from Tamada (2009). Data from two adsorbent experiments were used: braided adsorbent that was in $\mathrm{T}=20^{\circ} \mathrm{C}$ (red squares) and adsorbent stacks that were in $\mathrm{T}=30^{\circ} \mathrm{C}$ blue circles). The dotted blue line and solid red line represent the one-site ligand-saturation model fit to the data for the braided adsorbent and adsorbent stacks, respectively. . . . . . . . . . . . . . . .

2-7 Temperature dependance of kinetic parameters $\beta_{\max }$ (blue squares) and $K_{D}$ (red triangles) for (a) $38 \mathrm{H}$, (b) AF1, and (c) AI8 ORNL adsorbents as determined by experiments by PNNL. The blue and red lines indicate the linear fit of the $\beta_{\max }$ and $K_{D}$ data sets respectively Gill et al., 2014) . . . . . . . . . . . . . . . . .

2-8 Time-dependent uptake of uranium for the Al8 adsorbent fiber as predicted by $(2.15)$ for $\mathrm{T}=10^{\circ} \mathrm{C}$ (blue), $15^{\circ} \mathrm{C}$ (red) and $20^{\circ} \mathrm{C}$ (yellow).

2-9 Time-dependent uptake of uranium for the Al8 adsorbent fiber in a recirculating seawater flume with no light exposure (blue triangles) and with light exposure (red circles). The blue and red lines indicate the one-site ligand model fit by Equation (2.2), respectively (Park et al., 2016). The coefficients of the model, $\beta_{\max }$ and $K_{D}$ are presented with their 95\% confidence intervals. Adapted from Park et al. (2016). . . .

2-10 Time-dependent uptake of uranium for the AF1 adsorbent fiber in recirculating seawater flumes of various linear velocities. Adapted from Ladshaw et al. (2017). . . . . . . . . . . . . . . . . . . .

2-11 Relationship between the optimal values of the mass-transfer coefficient, $K$, and the linear velocities in the Hume experiments. Adapted from Ladshaw et al. (2017). . . . . . . . . . . . . . . . . . . . . .

3-1 Initial adsorbent concept with decoupling of mechanical and chemical requirements. Soft, inner adsorbent sphere is encased in tough, outer protective sphere. Outer sphere features holes to allow adequate seawater to adsorbent interior . . . . . . . . . . . . . . 50

3-2 Schematic of filament ball of uranium adsorbing fibers and shell enclosure model . . . . . . . . . . . . . . . . . . . . 
3-3 von Mises stress results for vertical loading of a (a) cube, (b) octahedron, (c) dodecahedron, (d) sphere with circular holes and (e) sphere with slotted holes shell enclosure . . . . . . . . . . . . . . . .

3-4 Factor of safety as a function of number of faces for the cube, octahedron, dodecahedron, sphere with circular holes and sphere with slotted holes shell enclosures . . . . . . . . . . . . . . . . . . . . . .

3-5 Solid models depicting cube shell enclosure with (a) large circular, (b) large square, (c) small circular, (d) rectangular slit hole geometries. .

3-6 Factor of safety as a function of hole geometry for the cube shell enclosure. Hole types A-D are shown in figure 3-5 and hole type $\mathrm{E}$ is a rotated version of hole type D. . . . . . . . . . . . . .

4-1 Solid models of six shell designs selected for testing. . . . . . . . . . .

4-2 Model of a protective shell enclosure. Each shell enclosure contained multiple tabs with corresponding slots to allow alignment of the two

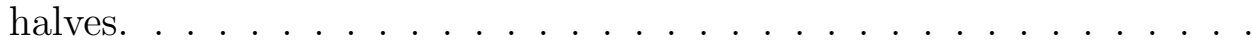

4-3 Six shell designs selected for testing. Each shell enclosure was fabricated in two halves. The upper half of each shell can be removed to allow for quick and easy sampling of the adsorbent. The outer diameter of all shell enclosures was $4 \mathrm{~cm}$. . . . . . . . . . . . . . . .

4-4 Diagram of the flume setup used to test the adsorbent enclosures. Fresh seawater is pumped in from a head tank using three tubes near the recirculation inlet. Overflow pipe ensures a constant water level of 9 in. A Finish Thompson DB8 Centrifugal Pump constantly recirculates the filtered seawater . . . . . . . . . . . . . . . . .

4-5 Flume tank setup with flow meter, centrifugal pump, and globe valve connected . . . . . . . . . . . . . . . . . 62

4-6 $\quad$ All six shells positioned in the flume tank. . . . . . . . . . . . . . . 62

4-7 Drag coefficient as a function of the Reynolds number for a solid sphere as predicted by Morrison, 2016 (solid blue line), a wiffle shell with diameter $50 \mathrm{~mm}$ (red stars) and $500 \mathrm{~mm}$ (yellow circles) as determined from CFD simulations, as well as those determined by Tow Tank drag experiments for a solid sphere (purple squares) and a wiffle ball (green diamonds) of diameter $100 \mathrm{~mm}$. The black circle indicates the experimental regime of the flume test described in this chapter. Tow Tank drag experiments were performed by Amanda Hamlet (Hamlet, 2017)

4-8 Photography showing the adsorbent in shells in the flume. . . . . . . 65

4-9 Time-dependent measurements of adsorption capacities (g element $/ \mathrm{kg}$ adsorbent) for several trace elements retained by the ORNL AI8 adsorbent exposed to filtered natural seawater in a flume in various shell enclosures, (a)-(f) (as shown in figure 4-3), and without any enclosure, (g). The black line drawn through the uranium adsorption data represents fitting to a one-site ligand model (equation (2.2)) . . . . . 
4-10 Time-dependent measurements of uranium adsorption capacity (g-U/kgadsorbent) for the flume experiment for the seven AI8 adsorbent braids enclosed by various shell designs (Enclosures A-F), as well as the results trom the control adsorbent in no enclosure and the results from a Hume experiment conducted in 2015 on Al8 fibers alone. The uranium adsorption capacity was normalized to a salinity of 35 psu. Curves drawn through the data represent fitting to a one-site ligand model (equation(2.2)). Figure 4-11 details the saturation capacity and halfsaturation times as predicted from the one-site ligand modeling . . .

4-11 The (a) saturation capacity, $\beta_{\max }$, (b) half saturation time, $K_{D}$, and (c) 56-day uranium uptake predicted by the one-site ligand model (equation (2.2)) . . . . . . . . . . . . . . . . .

4-12 The total uranium adsorbed at day 56 by the adsorbent fibers in each enclosure trom the flume experiment. . . . . . . . . . . .

5-1 Schematic of 5-MW NREL-OC3-Hywind offshore wind turbine used as the base of the design of SMORE where SWL is the still water Iine. The turbine has a total draft of $D_{\text {system }}=120 \mathrm{~m}$, an upper spar diameter of $d_{\text {upper }}=6.4 \mathrm{~m}$ and a lower spar diameter of $d_{\text {lower }}=9.4 \mathrm{~m}$ (Jonkman, 2010). . . . . . . . . . . . . . . . . .

5-2 Schematic of the Symbiotic Machine for Ocean uRanium Extraction (SMORE). In this design, large rollers are used to move multiple ballchain lengths of shell enclosures containing adsorbent fibers. . . . . .

5-3 Schematic of a ball-chain net. In this image, the number of ball-chain lengths $N_{b}=4 \ldots \ldots \ldots \ldots \ldots \ldots$

5-4 Schematic describing $\alpha$. in terms of the shells (orange) around the roller (black line). The blue line is the irregular polygon created by the shells and the spaces that make up the circumference of the roller and the red line is the regular polygon inscribed by the midpoints of the irregular polygon sides. Here $N_{s, r}=6$. . . . . . . . . . . . .

5-5 Schematic describing elution and regeneration process of polymer adsorbent . . . . . . . . . . . . . . . . . .

5-6 Model of small segment, $d s$, of the ball-chain under a lateral current load, $f(s)$, with constant apparent weight per unit length, $w$. The axis following the segment is given by $s, F$ is a sheer force, $T$ is the tension in the ball-chain, and $\theta$ is the angle the cable makes with the horizontal. 79

5-7 Three-dimensional model of SMORE for the a 1/10th physical scale version of the design case outlined in table 5.1. The entire 1/10th scale system is show on the turbine spar in (a); whereas (b) shows a close-up of the upper platform, and (c) is a close up of one of the roller subsystems (shown in purple). . . . . . . . . . . . . . . 
6-1 Three-dimensional model of 1/10th physical scale model for ocean testing of the SMORE design described in chapter 5. Both a stationary and continuous version of the design were fabricated and mounted to a wooden Hoat for ocean testing. . . . . . . . . . . . . . . . .

6-2 Stationary system design of (a) surface structure, (b) wheel assemblies, and (c) net and guide wires. . . . . . . . . . . . . 87

6-3 Fabricated surface structure of the continuous system design. . . . . . 88

6-4 Massachusetts Maritime Academy (MMA) (a) location in reference to Cape Cod Canal and Buzzards Bay, and (b) aerial view of MMA's campus with the location of the prototype test indicated by the white circle . . . . . . . . . . . . . . . . . .

6-5 Both prototypes mounted to the wooden float and moored to the end of the MMA dock during testing. . . . . . . . . . . . . . .

6-6 Shell designs for the testing of uranium adsorption fibers using (a) slotted holes and (b) circular holes. . . . . . . . . . . . . . .

6-7 Small section of net with white shells for adsorbent fibers. Adsorbent enclosure shells were alternated with orange placeholder shells used for mechanical testing. . . . . . . . . . . . . . .

6-8 Pre-weighed adsorbent mini braid. One to two of these mini braids were placed in each shell design on each of the two prototypes. . . . .

6-9 Map of MMA indicating the location of the wooden float with the prototypes, the piling to which most of the ocean sensors were attached, and the Xylem sensor. Note: the pier to which the Xylem sensor was ocated is fixed, not Hoating, on pilings and has water free flowing under it. The spot that the Xylem sensor was located was not protected by the pier and ship as the bird's eye view suggests. . . . . . . . . . . . . 93

6-10 Layout of instruments attached to piling at the end of the dock. . . . 94

6-11 Time-dependent uptake of uranium for the Al8 adsorbent fiber as predicted by 6.1 for $\mathrm{T}=10^{\circ} \mathrm{C}$ (blue line), $15^{\circ} \mathrm{C}$ (red line) and $20^{\circ} \mathrm{C}$ (yellow line $. \ldots \ldots \ldots \ldots . \ldots \ldots$

6-12 1ssues with the bottom roller that arose due to the inability to provide enough tension in the adsorbent net for prolonged periods of time. The adsorbent net was found to (a) slip off or (b) fall out of alignment with the roller . . . . . . . . . . . . . . . . . .

6-13 Major modifications made to the continuous system consisted of a (a) stationary 4 in diameter PVC pipe to replace the moving bottom roller and (b) a series of PVC guides along the length of the bottom support to keep the net separated and prevent tangling and misalignment due to strong currents. . . . . . . . . . . . . . . . . 
6-14 (a) Light intensity as measured by the top, middle, and bottom light sensors. (b) Temperature as measured from the U24 conductivity logger. (c) Salinity as measured from the Xylem EXO-2 Sonde salinity meter with dashed lines indicating $\pm 2 \hat{\sigma}$ where $\hat{\sigma}$ is the robust standard deviation. (d) Current as measured from the bottom current meter with the dashed lines indicating $\pm 20 \mathrm{~cm} / \mathrm{s}$. The gray rectangle indicates the period of the ocean test, October 20, 2016 - December 13, एण66. . . . . . . . . . . . . . . . .

6-15 Biofouling on the (a) stationary net and (b) continuously moving net at the end of the ocean test . . . . . . . . . . . . . .

6-16 Percent weight gain or loss in the adsorbent fibers before and after deployment at each sampling. . . . . . . . . . . . . . . .

6-17 Average percent weight gain or loss in the adsorbent fibers for each enclosure and system of all the time-dependent measurements with error bars indicating the standard deviation. . . . . . . . . . . . .

6-18 (a) $\mathrm{MnO}_{2}$ impregnated acrylic fibers used in this study and (b) the control cartridge housing some of these fibers through which a known volume of water was filtered . . . . . . . . . . . . . . . . . . .

6-19 The ashing process showing (a) one clump of $\mathrm{MnO}_{2}$ impregnated acrylic fiber in a container ready for ashing and (b) all samples in a muffle furnace prior to ashing. . . . . . . . . . . . . . .

6-20 Volume of water seen by $\mathrm{MnO}_{2}$ impregnated acrylic fibers in different enclosure types on different prototype systems as determined by ${ }^{226} \mathrm{Ra}$ count using $\gamma$-spectrometry. The control cartridge saw $120 \mathrm{~L}$. . . . . .

6-21 Time-dependent measurements of adsorption capacities (g element $/ \mathrm{kg}$ adsorbent) for several trace elements retained by the ORNL AI8 adsorbent used in the ocean test and enclosed in (a) shells with slotted and (b) circular holes of the stationary system, (c) shells with slotted and (d) circular holes of the continuous system, and (e) in the mesh bag on the stationary system. The black line drawn through the uranium adsorption data represents fitting to a one-site ligand model by (2.2).

6-22 Time-dependent measurements of uranium adsorption capacity (g U/kg adsorbent) for the AI8 adsorbent braids enclosed by the different shell designs on the two different systems, and enclosed only by a mesh bag (control). The uranium adsorption capacity was normalized to a salinity of $35 \mathrm{psu}$. Curves drawn through the data represent fitting to a one-site ligand model by (2.2). . . . . . . . . . . . . . . . .

6-23 The uranium (a) saturation capacity, $\beta_{\max }$, (b) half saturation time, $K_{D}$, and (c) uranium uptake predicted from the one-site ligand model for the adsorbent enclosed different shell designs on the two different systems, and enclosed only by a mesh bag (control). . . . . . . . . . .

6-24 The total uranium adsorbed at day 56 by the adsorbent fibers in each

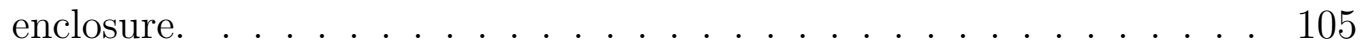


7-1 Floating spar wind turbine, similar to that of the NREL 5-MW wind turbine mounted on the OC3-Hywind spar (Jonkman et al., 2009; Jonkman, 2010) with incident waves of amplitude $A$ and frequency $\omega$. The motions of the turbine are described about the still water line (SWL). . . . . . . . . . . . . . . . . . . .

7-2 Three-dimensional solid models and fabricated designs for the reference floating wind turbine, (a) and (b), the SMORE design with the upper platform out of the water, (c) and (d), and the SMORE design with the upper platform submerged, (e) and (f) . . . . . . . . . . . . .

7-3 MIT Tow Tank where scale model hydrodynamic response tests were conducted of two designs of SMORE and the reference floating wind turbine . . . . . . . . . . . . . . . . .

7-4 Schematic of the experimental configuration. The wave maker on the left was excited at an amplitude, $A$, and frequency $\omega$. Two wave probes (white dots) downstream measured the amplitude incoming to the model (purple) for all tests. . . . . . . . . . . . . . . . .

7-5 Experimental setup of the excitation force test. One load cell was used to measure heave and two were used to measure surge. The difference in measurement of the two surge load cells and their distance from the SWL was used to determine the pitch torque. . . . . . . . . . . .

7-6 Experimental setup of the RAO test. A 9DOF accelerometer mounted to the tower of the turbine was used to measure heave, surge, and pitch motions . . . . . . . . . . . . . . .

7-7 Full-scale surge excitation force as determined by the load cell tests for small (blue squares) and large (red triangles) amplitude waves as compared to that numerically determined by Jonkman (2010) (black line) for the (a) reference floating wind turbine, (b) SMORE design with the upper platform above the water, and (c) SMORE design with the upper platform submerged. . . . . . . . . . . . . .

7-8 Full-scale heave excitation force as determined by the load cell tests for small (blue squares) and large (red triangles) amplitude waves as compared to that numerically determined by Jonkman (2010) (black line) for the (a) reference floating wind turbine, (b) SMORE design with the upper platform above the water, and (c) SMORE design with the upper platform submerged. . . . . . . . . . . . . . .

7-9 Full-scale pitch excitation torque as determined by the load cell tests for small (blue squares) and large (red triangles) amplitude waves as compared to that numerically determined by Jonkman (2010) (black line) for the (a) reference floating wind turbine, (b) SMORE design with the upper platform above the water, and (c) SMORE design with the upper platform submerged. . . . . . . . . . . . . . . 
7-10 Full-scale surge RAO as determined by the accelerometer tests for the (a) reference floating wind turbine, (b) SMORE with the upper platform above the water, and (c) SMORE with the upper platform submerged, for small (blue squares), medium (purple crosses) and large (red triangles) amplitude waves as compared to that numerically determined by Jonkman (2010) (black line). . . . . . . . . . . . . . .

7-11 Full-scale heave RAO as determined by the accelerometer tests for the (a) reference floating wind turbine, (b) SMORE with the upper platform above the water, and (c) SMORE with the upper platform submerged, for small (blue squares), medium (purple crosses) and large (red triangles) amplitude waves as compared to that numerically determined by Jonkman (2010) (black line). . . . . . . . . . . . . . . .

7-12 Full-scale pitch RAO as determined by the accelerometer tests for the (a) reference floating wind turbine, (b) SMORE with the upper platform above the water, and (c) SMORE with the upper platform submerged, for small (blue squares), medium (purple crosses) and large (red triangles) amplitude waves as compared to that numerically determined by Jonkman (2010) (black line). . . . . . . . . . . . . .

8-1 Timeline of the lifecycle of an adsorbent following the discounted cash How technique. . . . . . . . . . . . . . . 126

8-2 Loss in adsorbent uptake as a function of campaign length for the worst case degradation scenario, adapted from Byers and Schneider (2016b)

8-3 In the reference deployment strategy, the adsorbent is (a) braided into buoyant $60 \mathrm{~m}$ long lengths and (b) deployed across the seafloor. This strategy was (c) tested by the Japanese Atomic Energy Agency in the early 2000s (Tamada et al., 2006) . . . . . . . . . . . . . . . . .

8-4 The range of costs for both deployment schemes as a function of number of adsorbent uses . . . . . . . . . . . . . . . . . . 136

8-5 Breakdown of cost components contributing to the total cost of each deployment scheme for an intermediary case assuming no biofouling and worst case, time-dependent degradation. . . . . . . . . . . . . 136

8-6 Some cost components contributing to the mooring capital and operating costs for each deployment strategy. The category labeled "Other" includes other operating consumables and contingencies. . . . . . . . 137

8-7 Breakdown of adsorbent production costs. . . . . . . . . . . . 138

9-1 Typical wind turbine power output curve (PelaFlow Consulting, 2008). 142

$9-2$ SUSH GUU . . . . . . . . . . . . . . . . . . . . . . . . 145

9-3 Typical ocean currents dataset plot. . . . . . . . . . . . . . . . . 147

9-4 Typical ocean surface winds dataset plot. . . . . . . . . . . . . . . . . 148

$9-5$ Typical ocean sea surface temperature plot. . . . . . . . . . . . . . . 149

$9-6$ Typical suitability index plot. . . . . . . . . . . . . . . . . 150

9-7 Screenshot of the GU1 after the example inputs are entered. . . . . . 151

9-8 Screenshot of the GU1 after selected datasets are plotted. . . . . . . . 152 
9-9 Screenshot of the GU1 after selected datasets are plotted. . . . . . . . 152

10-1 Types of offshore wind turbine foundations. Monopile and tripod/jacket foundations are currently proven technologies. Floating structures have been using three main types of foundations adapted from the oil and gas industry: the Tension Leg Platform (TLP), semi-submersible (Semisub), and Spar Buoy (Spar). This figure is reproduced from the European Wind Energy Association (2013). . . . . . . . . . . . . . . . . . 157 


\section{List of Tables}

2.1 Composition range of seawater with respect to major and minor cations 34

2.2 One-site ligand saturation modeling of time-dependent measurements of uranium from How-through column tests for three adsorbent formulations obtained from ORNL (Kuo et al., 2016) . . . . . . . . . . . 41

2.3 One-site ligand saturation modeling of time-dependent measurements of uranium from tlume tests for two adsorbent formulations obtained from ORNL (Gill et al., 2016) . . . . . . . . . . . . . . . . . . . . . 42

3.1 Feasibility calculations for adsorbent filament ball. . . . . . . . . . . . 54

5.1 Example SMORE design . . . . . . . . . . . . . . . . . . . . . . 83

7.1 Scaling ratios for various physical parameters . . . . . . . . . . . . . 112

7.2 Scaling factors employed for wave model testing . . . . . . . . . . . . 112

7.3 Parameters for Excitation Force Experiment . . . . . . . . . . . . . . 116

7.4 Parameters for RAO Experiment . . . . . . . . . . . . . . . 118

8.1 Details of continuous SMORE design used in cost analysis . . . . . . 134

8.2 Input data used for cost-analysis . . . . . . . . . . . . . 135

8.3 Optimized deployment parameters leading to the minimum achievable uranium production cost. . . . . . . . . . . . . . . 135 


\section{Chapter 1}

\section{Introduction}

\subsection{Motivation}

The U.S. Energy Information Administration's recently released report, "International Energy Outlook 2013," projects that world energy consumption will grow by $56 \%$ between 2010 and 2040 (Energy Information Administration, 20113). With the global population forecasted to increase $33 \%$ by 2050 and over $50 \%$ by 2100 (Gerland et al, 2014), the rate of world energy consumption is expected to continue to rise over the century. At present, electricity production relies primarily on fossil fuels and is responsible for a large share of the carbon dioxide released to the atmosphere by human activities. The Intergovernmental Panel on Climate Change fourth assessment report stresses the importance of the reduction of anthropogenic greenhouse gas emissions in order to limit the extent of global warming (Intergovernmental Panel on Climate Change, 20(07). These objectives pose a unique challenge in which alternative power generation methods with low carbon emissions will be required to address global warming in the midst of increased global energy needs.

Given that one gram of ${ }^{235} \mathrm{U}$ can theoretically produce, through nuclear fission, as much energy as burning 1.5 million grams of coal (Emsley, 2001), nuclear fission has the potential to significantly reduce carbon dioxide emissions from power generation. Terrestrial supplies of uranium, however, are greatly limited. A 2014 study by the Organization for Economic Cooperation and Development estimated that at the current consumption rate the global conventional reserves of uranium (7.6 million tonnes) could be depleted in a little over a century (OECD Nuclear Energy Agency, 2016). Additionally, as reserves decrease, uranium mining will shift to lower quality sites leading to a higher extraction cost and even greater negative environmental impact. A growing nuclear power sector will need access to significant uranium reserves at a reasonable cost of extraction.

Fortunately, uranium is present in the ocean as uranyl ions at a low concentration

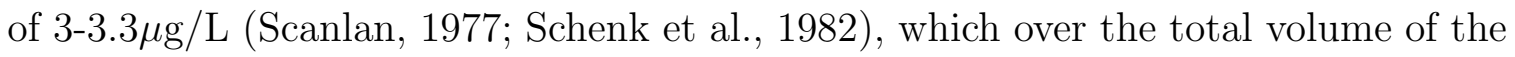
oceans amounts to approximately 4.5 billion tonnes of uranium - nearly a thousand times that of conventional terrestrial reserves (Tamada, 200.9). Finding a sustainable way to harvest uranium from seawater will provide a source of uranium for generations 
to come. It further gives all countries with ocean access a stable supply and eliminates the need to store spent fuel for potential future reprocessing, thereby also helping to address nuclear proliferation issues.

\subsection{Terrestrial Uranium}

Naturally occurring uranium is comprised of three main isotopes. Approximately $99.275 \%$ is ${ }^{238} \mathrm{U}$, another $0.711 \%$ is ${ }^{235} \mathrm{U}$ and about $0.005 \%$ is ${ }^{234} \mathrm{U}$. Terrestrial uranium exists as uranium ore and is primary extracted by being crushed and then leached with acids to produce 'yellowcake', which is a uranium oxide powder, $\mathrm{U}_{3} \mathrm{O}_{8}$. Terrestrial mining of uranium can take the form of in-situ leaching, open pit, and underground mining. Yellowcake is then converted into the gaseous uranium hexaflouride, $\mathrm{UF}_{6}$ for enrichment. Enrichment decreases the percentage of ${ }^{238} \mathrm{U}$ in the mixture, thereby concentrating the relative amount of ${ }^{235} \mathrm{U}$. One issue with deploying the enrichment of uranium is that highly enriched uranium can be used to produce nuclear weapons, raising issues of nuclear proliferation as more countries adopt nuclear technology into their energy portfolios.

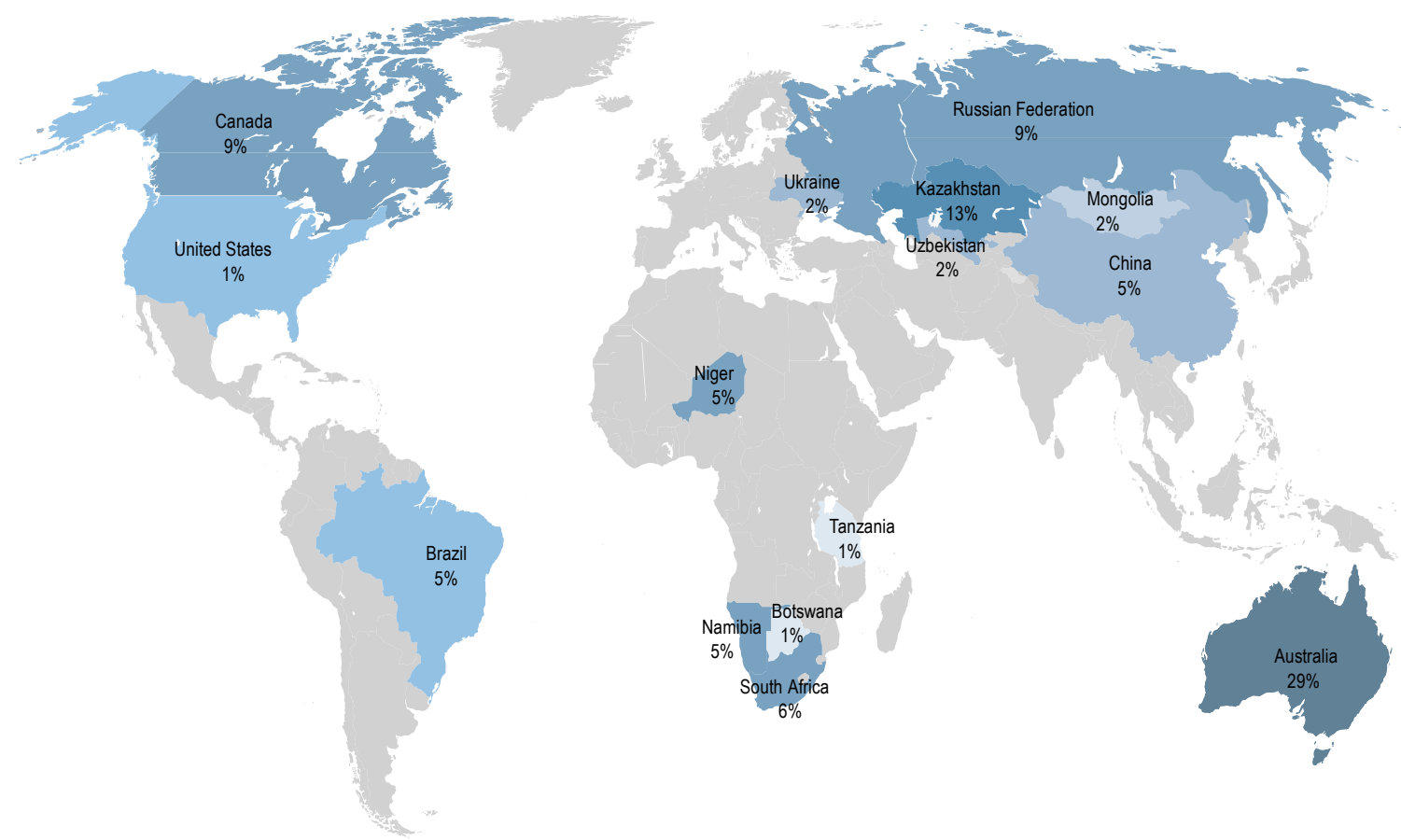

Figure 1-1: Global distribution of identified resources of uranium with a production cost of $<\$ 130 / \mathrm{kgU}$ among 15 countries that are either major uranium producers or have significant plans for growth of nuclear generating capacity. These 15 countries are endowed with $95 \%$ of the identified global resource base in this cost category - data as of January 1, 2015 (OECD Nuclear Energy Agency, 2016). Note that uranium with a production cost of $<\$ 50 / \mathrm{kgU}$ is considered the limit of "conventional" terrestrial resources. 


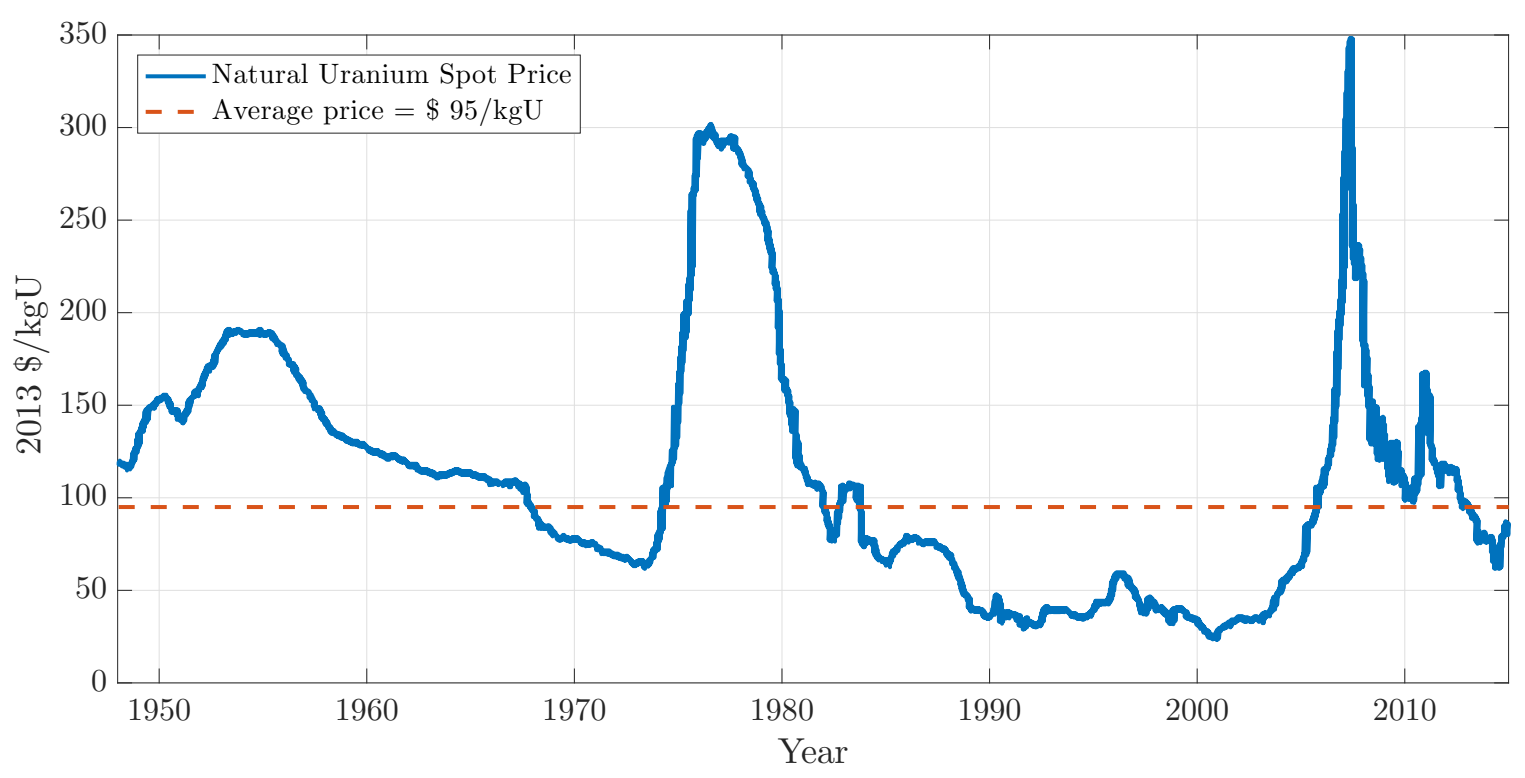

Figure 1-2: Uranium spot price from 1948-2015 in $2013 \$ / \mathrm{kgU}$. Adapted from Rothwell (2016). Uranium prices 1948-1972 from US Department of Energy ([98I), converted to monthly prices by interpolation from mid-year to mid-year, and converted to 2013 dollars using the Producer Price Index (Federal Reserve Bank of St. Louis, 2015). Prices January 1973-December 2006 are from Australian Bureau of Agricultural and Resource Economics and Sciences. Prices since 2006 were collected quarterly from the UxC website and converted to approximate monthly prices (Ux Consulting Company, LLC, 2015).

Terrestrial sources of uranium are not evenly distributed worldwide. As seen in figure प-1, which shows the global distribution of identified resources of uranium with a production cost of $<\$ 130 / \mathrm{kgU}$ from OECD Nuclear Energy Agency (2016), resources of uranium are greatly varied throughout the world. Note that uranium with a production cost of $<\$ 50 / \mathrm{kgU}$ is considered the limit of "conventional" terrestrial resources. Furthermore, $95 \%$ of the identified global resource base in this category is located in just 15 countries. As shown in the figure, the United States only accounts for $1 \%$ of the global distribution of uranium. Thus, from a supply security standpoint, the United States must rely heavily on political relations with those countries that have large quantities of uranium resources such as Australia (29\%), Kazakstan (17\%), Russia (9\%) and Canada (9\%), which can prove challenging.

As can be seen from figure [-2, which shows the spot price of uranium in 2013 $\$ / \mathrm{kgU}$, the spot price of natural uranium is extremely volatile. The 1968 peak price of $\$ 301 / \mathrm{kgU}$ ( $\$ 90 / \mathrm{kgU}$ in 1976 US dollars) was due to the fact that although private ownership of uranium began in the United States, the United States Atomic Energy Commission still maintained a monopoly on uranium enrichment services. The most recent peak in 2007 of $\$ 352 / \mathrm{kgU}$ ( $\$ 300 / \mathrm{kgU}$ in 2007 US dollars) has been attributed to the flooding of the Cigar Lake Mine, Saskatchewan, which has the largest undeveloped high-grade uranium ore deposits in the world. This created uncertainty about short-term future of the uranium supply (Mickey, 20108 ), as well as speculation about 
growing nuclear programs in India and China.

Furthermore, terrestrial mining techniques are often environmentally unsustainable, producing air pollution, contaminating ground water as well as surface water, and often releasing other radioactive elements such as radium and radon (OECD) Nuclear Energy Agency, 2014). With such unequally distributed terrestrial sources, unstable prices, and detrimental environmental impact, harvesting uranium from seawater would afford supply and cost security as well as environmental sustainability.

\subsection{Seawater Uranium Extraction}

Extraction of uranium from seawater has been researched for decades, with one of the first studies conducted by Davies et al. (1964) after World War II in an effort to secure uranium supply for Britain at a time when the production of uranium was uncertain. A recent review of uranium recovery technologies by Kim et al. (2013b) identified uranium adsorption by chelating polymers to be the most promising in terms of cost, adsorption capacity, and environmental footprint (Zhang et al., 20103; Seko et al, 20103; Anirudhan et al, 2017). Other techniques, including membrane filtration, coagulation, and precipitation were found to have issues such as high operating costs, durability, or toxicity (Kanno, 1.984; van Reis and Zydney, 2007); Tularam and llahee, 2007).

Chelating polymers allow for the passive extraction of uranium from seawater by adsorption. The polymers are first deployed in seawater and remain submerged until the amount of captured uranium approaches the adsorbent capacity. The length of time the fibers are submerged is known as the harvest period. At this point, elution is used to strip the uranium from the polymers. During this process, the adsorbent is immersed in acid solutions of increasing concentration to recover uranium and remove other elements that have bonded to the polymer. The adsorbent polymer may undergo a number of elution cycles before being regenerated by an alkali wash so that its functional groups are freed and the adsorbent can be reused. The output from the elution process undergoes purification and precipitation typically applied to mined uranium to produce yellowcake. Past work has focused on systems in which the adsorbent is brought back to shore for the elution process and redeployed afterward. These stand-alone intermittent operation systems, however, have significant practical and economic deployment challenges (Seko et al, 2003 ), and to date none of these systems have become economically viable.

Several of the polymer adsorbent system concepts have been subject to marine tests to evaluate performance, feasibility and cost-effectiveness. The Japanese Atomic Energy Agency (JAEA) first developed a system of buoy floated stacks of adsorbent fabric (figure [-3]). Due to the large weight of the mooring equipment, however, mooring operations were found to account for more than $70 \%$ of the cost of this concept (?Seko et al, 2003).

To address this problem, a buoyant braid adsorbent made of polyethylene fibers on a polypropylene trunk was proposed by Tamada et al. (2006) (figure [-4). This design was found to achieve a reduction of $40 \%$ of the cost of uranium recovery compared 


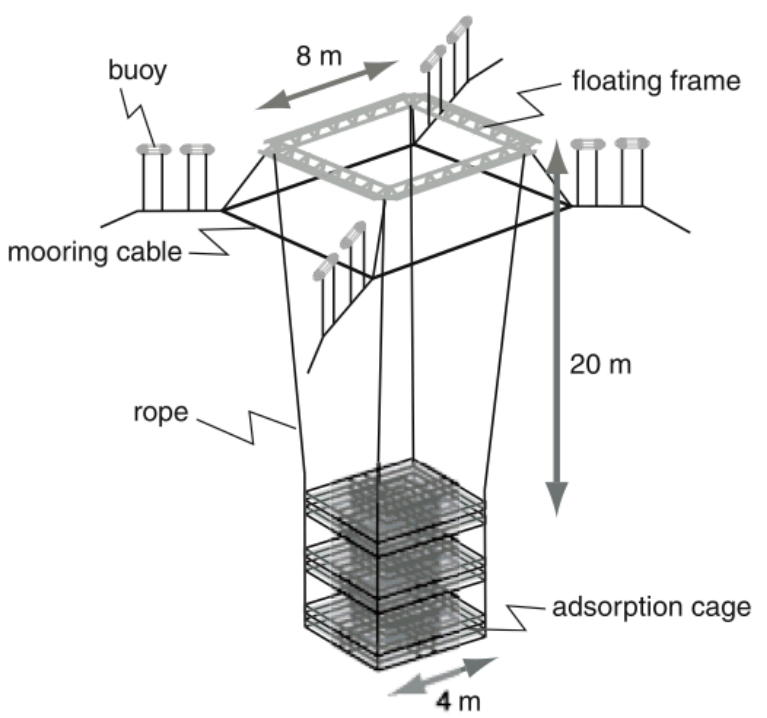

Figure 1-3: Uranium collection system for adsorbent stacks (Seko et al, 20(1)3).

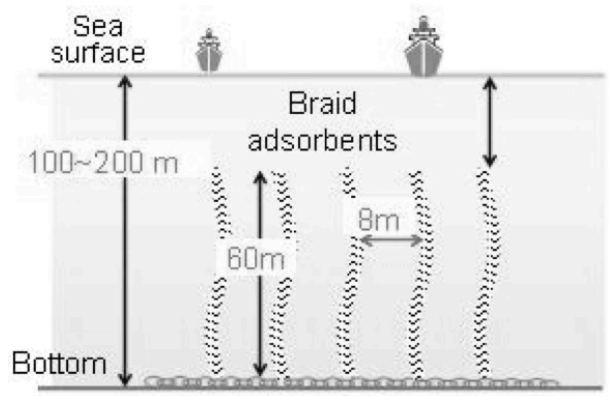

Figure 1-4: Uranium collection system for braid adsorbent (Wamada et al, 2006).

to the adsorbent stack system, resulting in an estimated uranium production cost of $\$ 1000 / \mathrm{kgU}$ (Tamada et al., 2006). An independent cost-analysis by Schneider and Sachde (2013) of the system yielded a production cost of $\$ 1230 / \mathrm{kgU}$. The difference in cost was mainly attributed to the consideration of a $5 \%$ degradation of adsorbent capacity per use cycle. Further sensitivity studies confirmed that the major cost drivers of such a system were the adsorbent capacity, number of recycles, and capacity degradation. For instance, if the capacity of the adsorbent was increased from $2 \mathrm{~kg}-$ $\mathrm{U} / \mathrm{t}$-ads to $6 \mathrm{~kg}-\mathrm{U} / \mathrm{t}$-ads and the number of recycles was increased from 6 to 20, with no degradation and unchanged adsorbent production costs, the uranium production cost would drop to $\$ 299 / \mathrm{kgU}$ (Schneider and Sachde, 2013). In comparison, the market price of uranium has ranged from a current low of $\$ 81 / \mathrm{kgU}$ to a peak of $\$ 300 / \mathrm{kgU}$ in 2007 when demand for nuclear power was higher. 


\subsection{Symbiotic Designs}

Previous studies show that a major cost driver of harvesting uranium from seawater is the mooring and recovery of the adsorbent (Schneider and Sachde, 20133). Based on this observation, Picard et al. (2014) pursued the development of a system which continuously takes the adsorbent from the ocean through an elution process and then returns it to the ocean. The autonomous nature of this design allows for control over the harvest period without requiring ships and labor to service the deployment. The system is designed to function attached to turbines of an offshore wind farm, thereby eliminating the offshore mooring cost while also increasing the energy output of the wind farm.

Figure $1-5$ shows the concept developed by Picard et al. (2014) in which a platform at the base of the wind tower supports a belt of adsorbent that loops in and out of the water. The belt slowly cycles through the seawater beneath the tower and through an elution plant located on the platform. The belt is weighted in the seawater by rollers which also space out the loops and prevent the belt from tangling. The proposed system was sized to collect 1.2 tonnes of uranium per year, a sufficient amount to supply $5 \mathrm{MW}$ of nuclear power. This would require $4 \mathrm{~km}$ of adsorbent belt for a total weight of 120 tonnes of adsorbent per wind turbine per year. To harvest enough uranium for a $1 \mathrm{GW}$ nuclear power plant would require 214 wind turbines and a total of over 25000 tonnes of adsorbent per year. Preliminary analysis conducted by Picard et al. (2014) on the adsorbent belt and structural design to determine the first order scaling laws for this concept indicate that such a system is technically feasible.

Picard et al. (2014) designed, built, and pool tested a 1/50th scale prototype to study the feasibility of a symbiotic uranium harvester and wind turbine system, with cables replacing the absorbent net (figure [-6). This prototype demonstrated that such a system could mechanically work and that weighted rollers could be configured such that the system would be stable and the loops of adsorbent belt would be roughly the same length as the belt cycles through the system. The test also indicated that the system could withstand moderate currents when a bottom roller system was used to maintain tension in the cables and keep them separated.

The rational behind coupling a uranium harvester with an offshore wind turbine is that the development of offshore wind or uranium harvesting by itself bears a high capital cost for the structures, but if the mooring function can be shared, the overall cost for each will be lower. An independent cost analysis of the design by Byers et al. (2016) of the system proposed by Picard et al. (2014), referred to in the rest of this thesis as the Wind and Uranium from Seawater Acquisition symBiotic Infrastructure (WUSABI), yielded a uranium production cost of $\$ 400-\$ 850 / \mathrm{kgU}$. As compared to an updated kelp deployment scheme (Tamada et aL, 20106; Schneider and linder, 2014) which yielded a uranium production cost of $\$ 450-\$ 890 / \mathrm{kgU}$, it was found that the WUSABI scheme could achieve savings of up to $11 \%$, due mainly to the fact that such a symbiotic scheme has lower deployment and mooring operations costs, as hypothesized. This can be clearly seen in figure [-7 which shows the cost breakdown for an example deployment scenario in which no biofouling affects on the uranium adsorbent are accounted for and time-dependent degradation of the adsorbent with 


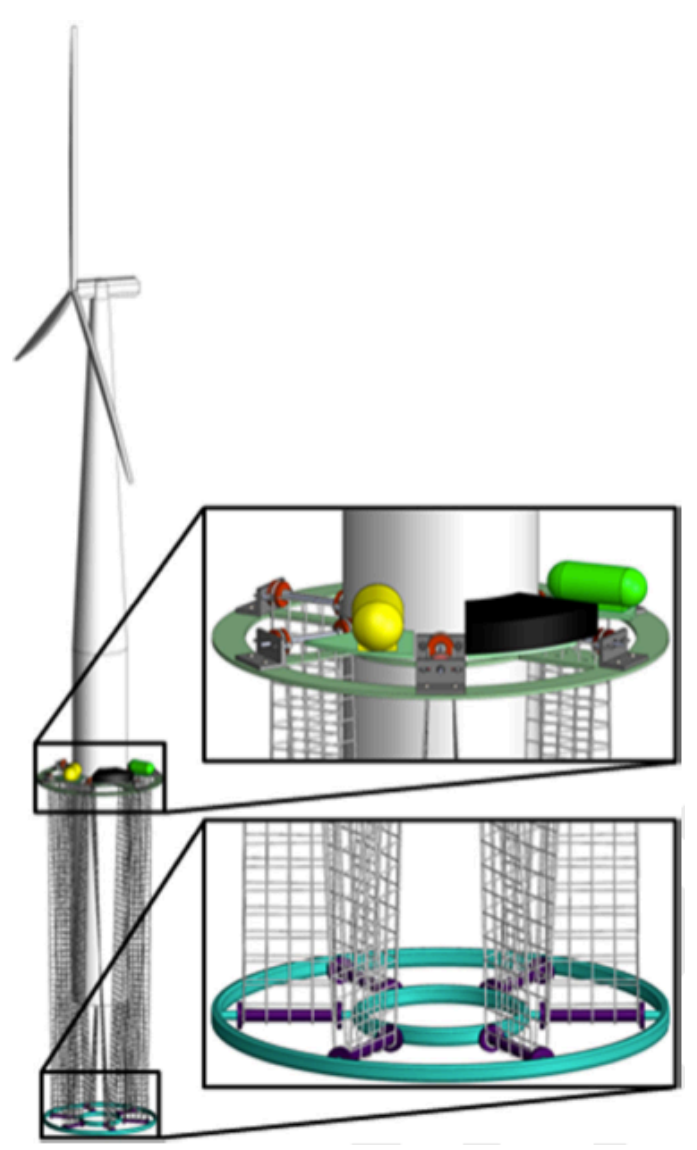

Figure 1-5: Three-dimensional view of continuous uranium recovery system with adsorbent belt looped around the turbine mast proposed by Picard et all (2014). The elution plant is housed on the upper platform out of the seawater.

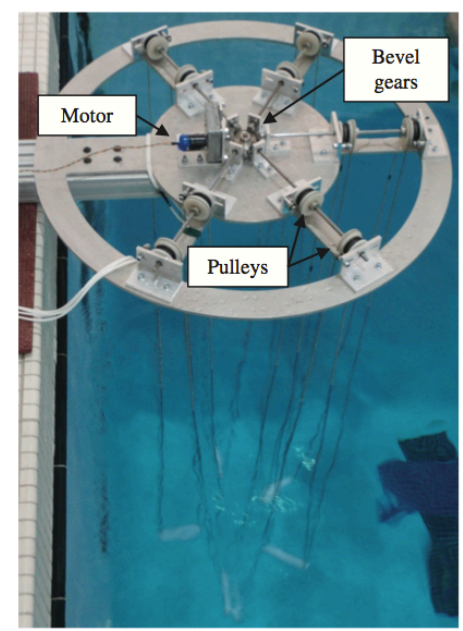

Figure 1-6: 1/50th scale model of Picard et all (2014) being tested in a pool.

subsequent elution treatments is assumed (Byers et al., 2016). Chapter $\nabla$ further details the cost-analysis method used for analyzing the reference kelp deployment 


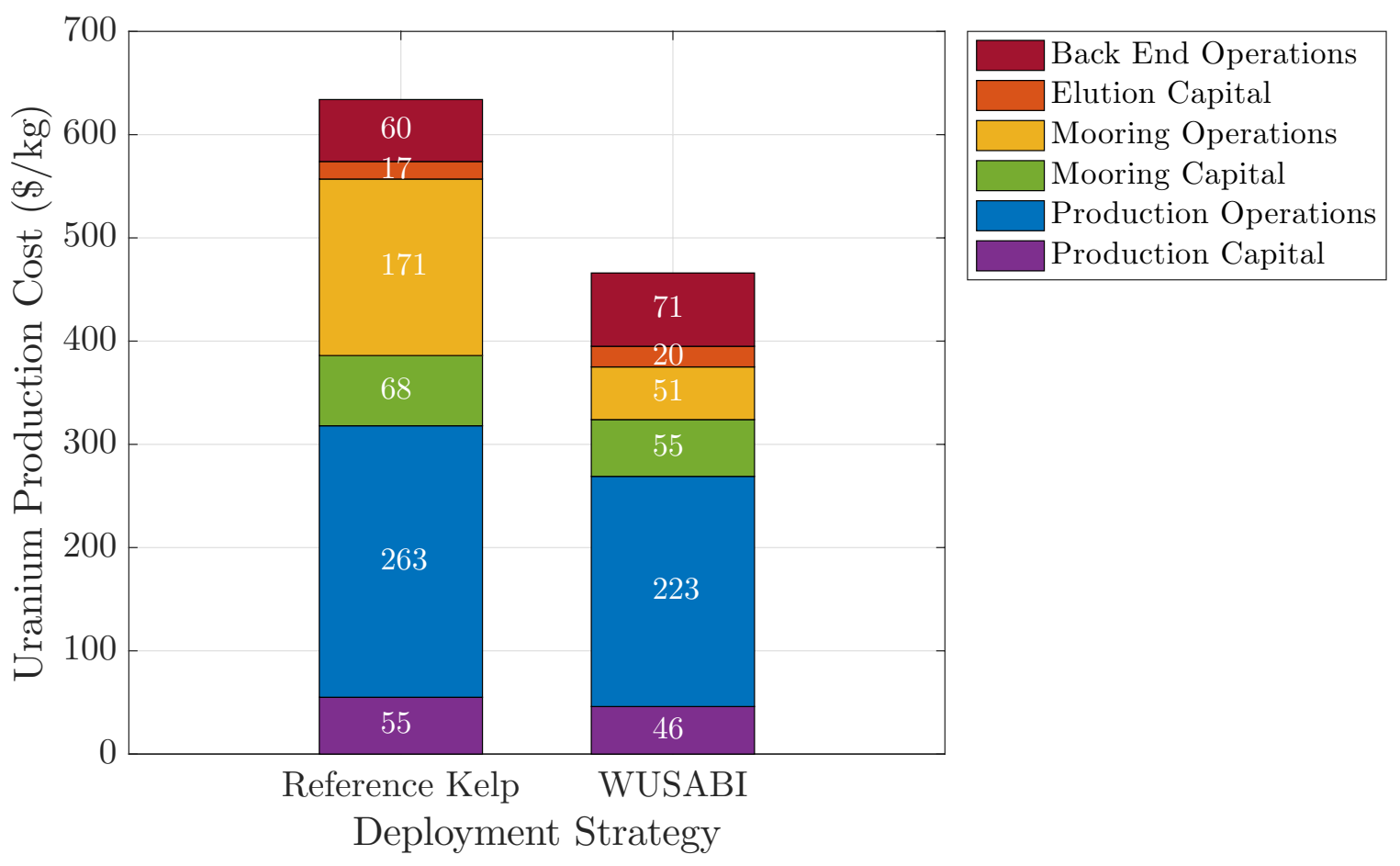

Figure 1-7: Cost analysis of WUSABI as initially designed by Picard et al. (2014), compared to the reference kelp deployment strategy (Byers et al., 2016).

scheme, WUSABI, and the designs produced as part of this thesis.

\subsection{Feasibility}

Why offshore wind turbines? Given the low concentration of uranium in seawater, the harvesting of 240 tonnes of uranium necessary to power a $1 \mathrm{GW}$ power plant for one year requires an onshore plant that can pump $5100 \mathrm{~m}^{3} / \mathrm{s}$ of seawater through the plant, or approximately $160 \mathrm{~km}^{3}$ of seawater per year. If the seawater for the onshore harvesting system was also used to provide cooling water in addition to nuclear fuel for the $1 \mathrm{GW}$ nuclear power pant, $5100 \mathrm{~m}^{3} / \mathrm{s}$ of flow represents about $21 \mathrm{GW} /{ }^{\circ} \mathrm{C}$ of cooling potential or enough cooling capacity for $315 \mathrm{GW}$ of electric power generation, which is about 150 times what is needed for the power plant (Union of Concerned Scientists, (201). Given the amount of water that would need to be pumped for an onshore system, which is far more than is required to cool a nuclear reactor, it is more cost-effective to forgo active pumping and instead locate the uranium harvesting system offshore using the ocean currents to flow water past the device.

Desalination plants routinely pump seawater onshore, generating a supply of freshwater and a brine mixture that has a concentration of at least 3 times that of seawater (Fravell, 2014). The feasibility for harvesting uranium from the brine produced from a desalination plant was also investigated. To harvest 240 tonnes of uranium required to power a $1 \mathrm{GW}$ power plant would require $53 \mathrm{~km}^{3}$ of brine flow per year, which would be produced from a desalination plant that generates 77 billion gallons 
of freshwater per day. This plant would have to be able to generate 285 times more freshwater than the largest desalination plant in the world: Ras al-Khair in Saudi Arabia, which produces 270 million gallons of freshwater per day. The sheer volume of water required for the case of harvesting uranium from brine further emphasizes the cost-effectiveness of an offshore uranium harvesting system.

\subsection{Thesis overview}

This thesis builds upon the work of Picard et al. (2014), and further focuses on the integration of a uranium harvesting system into an offshore wind turbine tower, taking into account various properties and characteristics of the adsorbent polymer as well as the structure of the offshore wind turbine.

The characteristics of the adsorbent polymers that will be referenced and utilized in the designs investigated in this thesis are presented in chapter 2. The uranium uptake of the adsorbent has been shown to be sensitive to both temperature and biofouling. Furthermore, a model is developed by which the uranium uptake of the adsorbent can be optimized by tuning mechanical parameters of the system for a specific adsorbent.

Chapter 3 details some of the mechanical properties of the adsorbent, which is found to be inherently weak in tensile strength and durability. This chapter also presents a strategy in which the mechanical and chemical requirements of the uranium harvester may be decoupled by using a permeable, hard outer shell to protect an adsorbent fiber interior.

To determine if such shell enclosures would impede the uptake of uranium by the

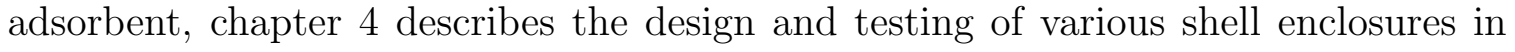
a time series flume experiment. In the experiment, six shell enclosure designs are developed and tested in a recirculating flume. A control adsorbent with no shell enclosure is also included in the experiment. The results indicate that there is no significant affect on the uptake of uranium by the shell enclosures.

Chapter 5 builds on the promising results of the flume experiment to detailed the design of a Symbiotic Machine for Ocean uRanium Extraction (SMORE) that utilizes shell enclosures that decouple the mechanical and chemical requirements of the system. The design incorporates the use of shell enclosures fabricated into a ball-chain net that be actuated by rollers to move the adsorbent net as desired. The design also utilizes multiple subsystems to reduce the risk of all systems failing at once. A design tool is developed to allow for the quick examination of the design space for a variety of input parameters such as turbine size, adsorbent type, ocean current speed and motion of the ball-chain net.

To investigate whether a stationary or continuously moving ball-chain net would be beneficial for uranium uptake (the rationale behind the latter being that a continuously moving system would induce more water flow to the adsorbents), a 56-day ocean test of two 1/10th physical scale prototypes was conducted and is described in chapter [6. In collaboration with Woods Hole Oceanographic Institute, a novel method of determining water flow using radium adsorbing fibers is employed. The 
results indicate that the continuous system increases water flow to the adsorbents, as hypothesized, while also reducing biofouling on the shells, which could result in a higher uranium uptake.

Based on the results of the ocean test, chapter $\square$ presents the testing of two $1 / 150$ th scale SMORE designs that utilize a continuously moving ball-chain net. The scale models are tested in the MIT Tow Tank to determine the resulting hydrodynamic response of the system, which is compared to that of an unmodified floating wind turbine. The tests show that the addition of the uranium harvester to the floating offshore wind turbine does not have a significant affect on the hydrodynamic response of the system.

Chapter 8 details the cost-analysis of a full-scale SMORE design. It utilizes a discounted cash flow technique to follow the costs of a unit mass of adsorbent accrued throughout its lifetime, as was done in previous cost analyses (Schneider and Sachde, 2013; Byers and Schneider, 2016a; Byers et al., 2016; Haji et al., 2017). The results show the production cost of uranium from seawater using a SMORE deployment strategy ranges from $\$ 313-\$ 593 / \mathrm{kgU}$. Compared to the cost of seawater uranium using the reference deployment strategy (Tamada et al., 20106; Schneider and Linder, 2014), in the best case scenario using a SMORE deployment strategy reduces the cost of seawater uranium by $27 \%$ to $\$ 313 / \mathrm{kgU}$, within $4.3 \%$ of the peak uranium cost of $\$ 300 / \mathrm{kgU}$ in 2007.

The Seawater Uranium Suitability of Harvesting Index (SUSHI) is developed in chapter 9 to examine the locations and conditions where a SMORE device could be implemented. A graphical user interface tool for quick analysis of worldwide locations is also described. The tool takes into account important properties such as ocean temperature, wind speed, and ocean current speed.

Chapter [0 provides a summary of the contributions of this thesis, and also outlines outstanding questions and identifies opportunities for future research. 


\section{Chapter 2}

\section{Adsorbent Characteristics}

This chapter discusses key characteristics of the adsorbent utilized in the design process of the development of a symbiotic system to harvest uranium from seawater.

\subsection{Adsorbent overview and properties}

The system currently studied by a nationwide consortium of national laboratory and university partners, of which this project is a part, utilizes an adsorbent that consists of a backbone of high density polyethylene co-grafted with an amidoxime ligand to afford uranium affinity. Additionally, a co-monomer is co-grafted in order to increase the adsorbent's hydrophilicity.

These continuous adsorbent polyethylene fibers can be braided around a porous polypropylene float which can be made into long lengths (Tamada et al, 2006). After deployment in seawater and elution to remove trace metals, including uranium, the functional groups on the adsorbent polymer need to be regenerated with an alkaline solution before they can be redeployed.

\subsection{Seawater uranium adsorption}

Adsorption of uranium from seawater is challenging because uranium mainly exists in the ocean as the stable tricarbonate complex, $\left[\mathrm{UO}_{2}\left(\mathrm{CO}_{3}\right)_{3}\right]^{-4}$ in very low concentrations of about $3.3 \mu \mathrm{g} / \mathrm{L}$ (Davies et al., 1964; Scanlan, 1977; Schenk et al, 1982) in the presence of relatively high concentrations of other ions, such as sodium, potassium, calcium, and some transitional metal ions (Davies et al., 1964; Scanlan, 1977; Schenk et al., 1982; Choppin, 1989). Owens et al. (2017) also found that the concentration of ${ }^{238} \mathrm{U}$ present in the ocean is directly proportional to the salinity of seawater by

$$
{ }^{238} \mathrm{U}( \pm 0.061)=0.100 \times \mathrm{S}-0.326
$$

where $\mathrm{S}$ is the salinity and the concentration of ${ }^{238} \mathrm{U}$ is in $\left(\mathrm{ng} \mathrm{g}^{-1}\right)$. The concentrations of various metal ions in seawater, including $\left[\mathrm{UO}_{2}\left(\mathrm{CO}_{3}\right)_{3}\right]^{-4}$, are given in table [2.] (EISayed et al., 2014). 
Table 2.1: Composition range of seawater with respect to major and minor cations

\begin{tabular}{|ll|}
\hline Ion & Seawater concentration $(\mathrm{g} / \mathrm{L})$ \\
\hline $\mathrm{Na}^{+}$ & $10.77-26.70$ \\
$\mathrm{Ca}^{2+}$ & $0.23-0.50$ \\
$\mathrm{~K}^{+}$ & $0.21-0.50$ \\
$\mathrm{Sr}^{2+}$ & $0.008-0.113$ \\
$\mathrm{Mg}^{2+}$ & $1.29-1.77$ \\
$\mathrm{Li}^{+}$ & 0.17 \\
$\mathrm{Rb}^{+}$ & 0.12 \\
$\mathrm{Ba}^{2+}$ & 0.03 \\
$\mathrm{Cu}^{2+}$ & 0.003 \\
{$\left[\mathrm{UO}_{2}\left(\mathrm{CO}_{3}\right)_{3}\right]^{-4}$} & 0.0033 \\
\hline
\end{tabular}

Adsorbents with amidoxime groups allow for binding of uranyl ions, $\mathrm{UO}_{2}{ }^{2+}$, however the first step in this process requires the dissociation of $\left[\mathrm{UO}_{2}\left(\mathrm{CO}_{3}\right)_{3}\right]^{-4}$, considered to likely be the rate limiting step (D)as et al, 20108, 20(1)9). Kinetic analysis of the model displacement reaction confirmed that the rate-determining step in the extraction process, was both the the complexing of uranyl by amidoxime group coupled with the dissociation of the carbonate group from the uranyl tricarbonate complex $\left[\mathrm{UO}_{2}\left(\mathrm{CO}_{3}\right)_{3}\right]^{-4}$ Wang et al. (2014).

Metal ions may bind to amidoxime in a variety of ways. Three possible binding motifs are shown in figure $[2-1$. In the first motif, $[2-[$ a, the metal ion is bound to the oxygen atom. In the second, $\mathbb{2}-\mathbb{D}$, the metal ion is bound to a five-membered chelate ring with oxygen and nitrogen donor atoms. In the third, $[2-\mathbb{c}$, the metal ion is bound with the $\mathrm{N}-\mathrm{O}$ bond of oximido. $\mathrm{UO}_{2}{ }^{2+}$ has been founded to be almost exclusively observed to bind in the third (Vukovic et al., 2012; Kelley et al., 2014). Furthermore, extremely fast kinetics in the uptake of other metal ions by the amidoxime adsorbent suggests that the major cations are not binding solely to amidoxime ligands, but rather primarily to nonspecific sites (e.g., $\mathrm{COO}-$ ) on the adsorbent, possibly due to their very high concentration in seawater (Kuo et al., 2016).
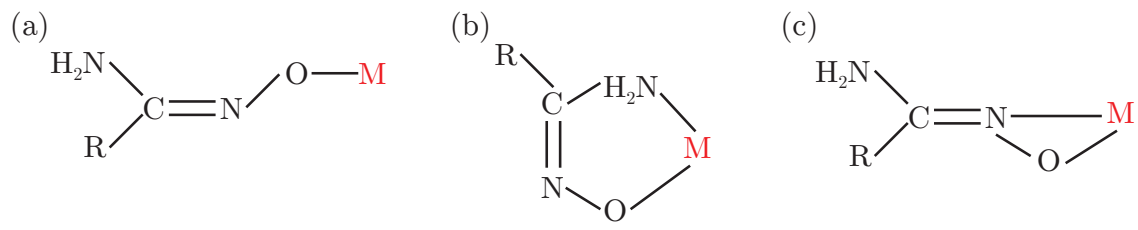

Figure 2-1: Three possible bonding motifs between amidoxime and metal ions (indicated by the red M): (a) the metal ion is bound to the oxygen atom, (b) the metal ion is bound to a five-membered chelate ring with oxygen and nitrogen donor atoms, and (c) the metal ion is bound with the $\mathrm{N}-\mathrm{O}$ bond of oximido. 


\subsection{Elution and regeneration}

Previous studies considered an acidic elution bath, such as 1.0M hydrochloric acid (Schneider and Sachde, 2013; Picard et al., 2014). After this elution, the adsorbent is washed in a solution of $\mathrm{KOH}$ to regenerate the active functional groups for reuse (T. Suzuki and Oguma; Seko et al, 201(14). In this method, however, this acidic elution process leads to significant degradation of the adsorbent with each reuse due to the deterioration of the sorbent caused by acid hydrolysis. It has been found to result in up to $20 \%$ loss of initial capacity in a lab setting (Pan et al, 2014), though in the ocean could be considerably greater.

Recent research by Pan et al. (2017) has indicated that this degradation may be mitigated or removed altogether by the replacement of the acidic elution bath with a bicarbonate solution. In this process, a mixture of sodium carbonate and hydrogen peroxide is used to elute uranium from an amidoxime-based polymer adsorbent. An added benefit is that the carbonate- $\mathrm{H}_{2} \mathrm{O}_{2}$ elution does not require any reconditioning step. Instead, rinsing with water is sufficient to regenerate the sorbent for reuse. Further work is being done on other basic elution methods that utilize potassium bicarbonate, which have also proven to reduce the degradation of the adsorbent.

\subsection{Adsorbent behavior}

In order to design a suitable uranium extraction system, a good understanding of the adsorbent behavior is necessary. Using the AF1 adsorbent developed at Oak Ridge National Laboratory (Gill et al, 2016) as as a reference, key characteristics of the adsorbent are presented here, namely the recovery rate of uranium, the adsorbent degradation as a result of the elution process, and the optimization of the main mechanical parameters of immersion time and number of reuses.

\subsubsection{Uptake}

According to Saito et al. (2014), the uranium complexation with amidoxime is presumed to follow a one-site ligand saturation model, where the uranium uptake, $C_{0}$, after a certain exposure time in days, $t$, is given by

$$
C_{0}=\frac{\beta_{\max } t}{K_{D}+t}
$$

where $\beta_{\max }$ is the saturation capacity in $\mathrm{kg}-\mathrm{U} / \mathrm{t}$-ads, and $K_{D}$ is the half-saturation time in days, both properties of the adsorbent used from Gill et al. (2016). After initial conditioning, the adsorbent capacity is degraded by a marginal amount. The ratio of adsorbent capacity after initial conditioning pre-deployment to theoretical capacity is taken to be $C_{\text {ratio }}=90 \%$ for this study. Therefore, the actual adsorbent capacity is

$$
C=C_{\text {ratio }} C_{0}=C_{\text {ratio }} \frac{\beta_{\text {max }} t}{K_{D}+t}
$$



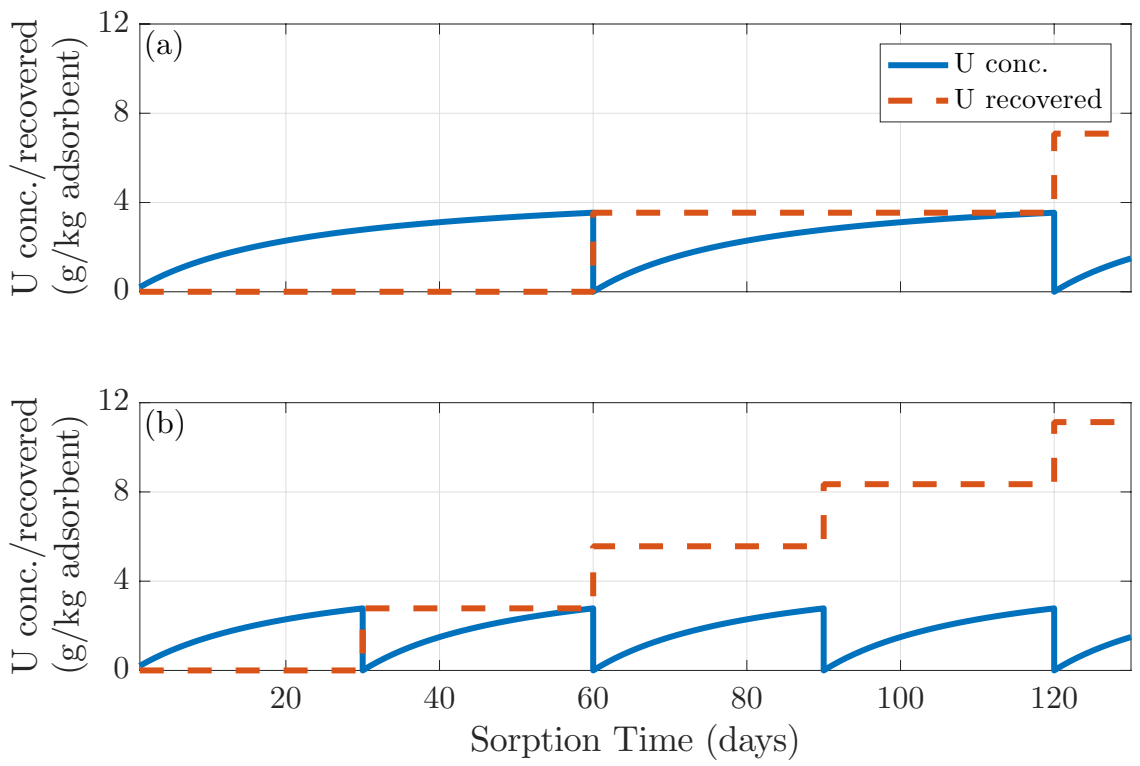

Figure 2-2: Adsorption kinetics. Resulting uranium concentration and uranium recovering (g/kg-ads) for a harvest period of (a) 60 days and (b) 30 days for the AF1 adsorbent.

Due to the kinetics of the adsorbent, the recovery rate of uranium of the adsorbent can be increased by shortening the harvest period. For instance, as shown in figure [2-2, when the period of uranium recovery from the adsorbent is reduced from 60 to 30 days, the amount of uranium collected over 120 days increases from $7 \mathrm{~g}-\mathrm{U} / \mathrm{kg}$-ads to $11 \mathrm{~g}-\mathrm{U} / \mathrm{kg}$-ads.

Taking $t=T_{h}$ be the harvest period, the rate of uranium recovery, $R$, is defined as follows,

$$
R=\frac{C\left(T_{h}\right)}{T_{h}}=\frac{C_{\text {ratio }} \frac{\beta_{\max } T_{h}}{K_{D}+T_{h}}}{T_{h}} .
$$

The recovery rate reaches a maximum as the harvest period approaches zero:

$$
\lim _{T_{h} \rightarrow 0} R=\frac{C_{\text {ratio }} \frac{\beta_{\max } T_{h}}{K_{D}+T_{h}}}{T_{h}}=\frac{C_{\text {ratio }} \beta_{\max }}{K_{D}} .
$$

The variation of the uranium recovery rate with the harvest period for the AF1 adsorbent is shown in figure [2-3]. The final choice of harvest period is a compromise between obtaining the highest recovery rate and minimizing damage to the adsorbent from more frequent elution.

\subsubsection{Adsorbent Degradation}

Experimental observation has shown that adsorbents can lose as much as $20 \%$ of their initial capacity over five adsorption/elution cycles (Seko et al., 2004). It is believed that exposure to highly concentrated acid causes damage to the functional groups 


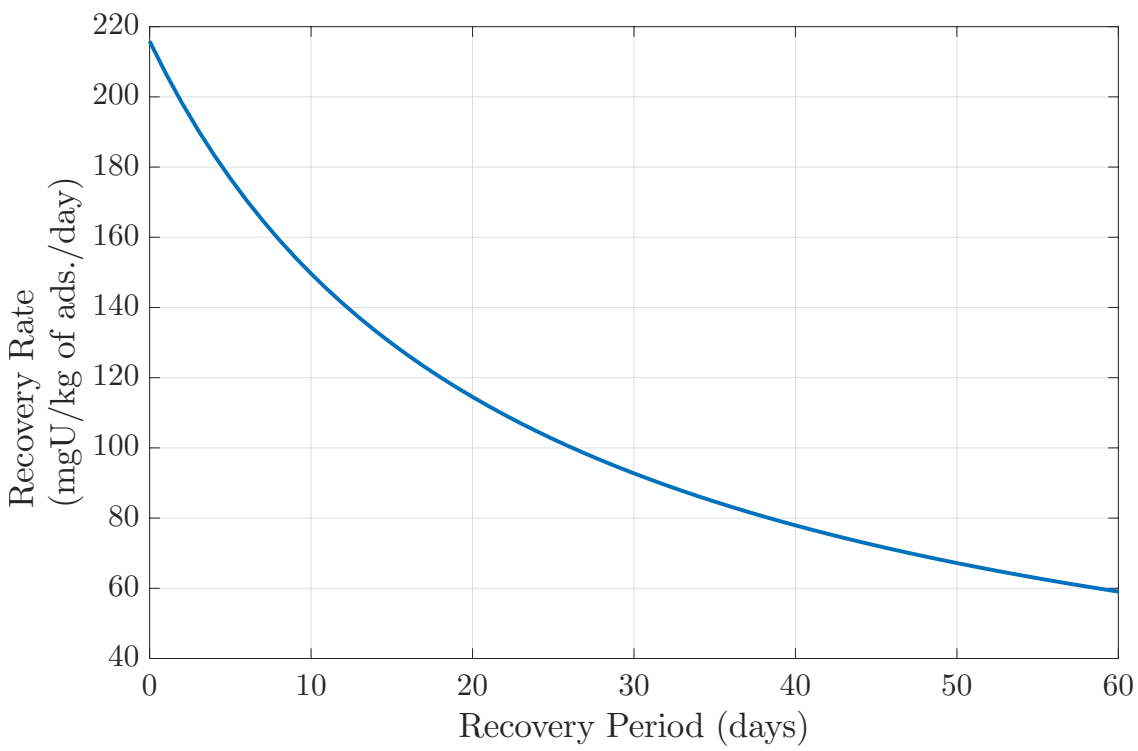

Figure 2-3: Recovery rate of uranium, $R$, as a function of the harvest time, $T_{h}$, for the AF1 adsorbent.

of chelating polymer adsorbent thus reducing their capacity. To model degradation during elution, it can be assumed that the time the adsorbent is exposed to the acid solution and the solution $\mathrm{pH}$ remain constant regardless of the recovery period. This is indeed necessary to make sure that all of the uranium is extracted from the adsorbent. Consequently, the relative loss of adsorbent capacity is assumed to be constant at each elution cycle since the damage to the adsorbent is expected to be the same.

As shown in (Picard et al., 2014), the capacity of the adsorbent after $n$ elution cycles can be written as

$$
C_{n}=C(1-d)^{n}
$$

Additionally, the average capacity of the adsorbent over $n$ adsorption/elution cycles can be calculated using a geometric progression:

$$
\bar{C}=\frac{1}{n} \sum_{k=0}^{n-1} C(1-d)^{k}=\frac{C}{n}\left[\frac{1-(1-d)^{n}}{d}\right]
$$

\subsubsection{Mechanical Parameters}

From $([2.3)$ and $([2.6])$, it is clear that the two mechanical parameters involved in the recovery of uranium are the time of exposure of the adsorbent to seawater, $t$, and the number of elution cycles of the adsorbent before replacement, $n$. After one cycle, the amount of uranium adsorbed in $\mathrm{g}$ - $\mathrm{U} / \mathrm{kg}$-ads, $\Gamma_{1}$, after a harvesting time of $t_{1}$, is given by 


$$
\Gamma_{1}=C_{\text {ratio }} \frac{\beta_{\max } t_{1}}{K_{D}+t_{1}}
$$

However, for every cycle thereafter, the degradation of the adsorbent becomes a factor. For instance, the amount of uranium adsorbed in $\mathrm{g}-\mathrm{U} / \mathrm{kg}$-ads after two cycles, $\Gamma_{2}$, each with a harvest time of $t_{1}$ and $t_{2}$ respectively is

$$
\Gamma_{2}=C_{\text {ratio }} \frac{\beta_{\max } t_{1}}{K_{D}+t_{1}}+C_{1} \frac{\beta_{\text {max }} t_{2}}{K_{D}+t_{2}},
$$

where $C_{1}$ is the capacity of the adsorbent after one elution cycle. As a result, $\Gamma_{2}$ becomes

$$
\Gamma_{2}=C_{\text {ratio }} \frac{\beta_{\max } t_{1}}{K_{D}+t_{1}}+C_{\text {ratio }} \frac{\beta_{\text {max }} t_{2}}{K_{D}+t_{2}}(1-d) .
$$

Following this procedure, assuming that the harvest time for each cycle is the same, that is $t_{1}=t_{2}=\cdots=t_{n}=t$, the amount of uranium adsorbed in $\mathrm{g}-\mathrm{U} / \mathrm{kg}$-ads after $n$ cycles, $\Gamma_{n}$, is

$$
\Gamma_{n}=C_{\text {ratio }} \frac{\beta_{\max } t}{K_{D}+t}\left[1+(1-d)+(1-d)^{2}+\cdots+(1-d)^{n-1}\right] .
$$

For this geometric progression, the amount of uranium adsorbed in g-U/kg-ads after $n$ cycles, $\Gamma_{n}$, becomes

$$
\Gamma_{n}=C_{\text {ratio }} \frac{\beta_{\max } t}{K_{D}+t}\left[\frac{1-(1-d)^{n}}{d}\right] .
$$

From ( $(2.12)$, it is clear that if the chemical properties of the adsorbent are fixed $\left(C_{\text {ratio }}, \beta_{\max }, K_{D}\right.$, and $\left.d\right)$, the harvest time, $t$, and the number of elution cycles, $n$, are the mechanical parameters that determine $\Gamma_{n}$, the amount of uranium adsorbed in $\mathrm{g}$ - $\mathrm{U} / \mathrm{kg}$-ads after $n$ cycles.

\subsubsection{Sorption Optimization}

The soprtion process can be mechanically optimized by using ([2.12) and the chemical parameters for the AF1 adsorbent from (Gill et al., 2016):

- Saturation capacity: $\beta_{\max }=5.421 \mathrm{~kg}-\mathrm{U} / \mathrm{t}$-ads,

- Half-saturation time: $K_{D}=22.6$ days,

- Degradation per cycle: $d=5 \%$.

The functional requirements of the symbiotic system require that 1.2 tonnes of uranium are harvested annually, therefore the parameter space can be further analyzed to highlight the combinations of harvest time and elution cycles that are feasible, as shown in figure 2-4. Additionally, the amount of adsorbent required on the system as a function of $t$ and $n$ can also be determined, the results of which are shown in figure 


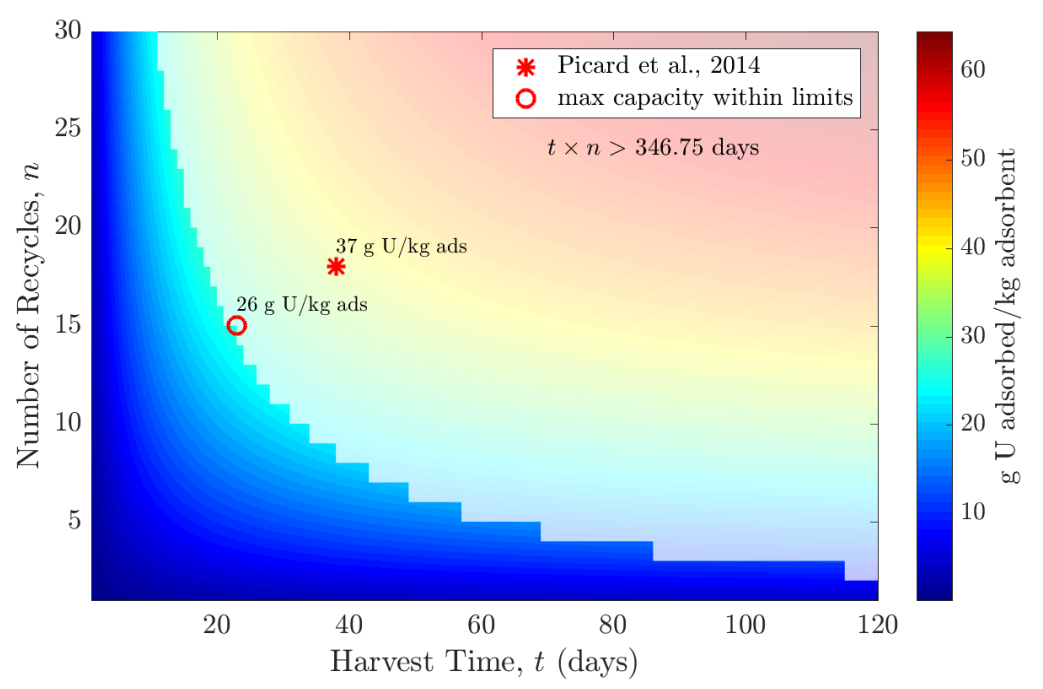

Figure 2-4: Amount of uranium adsorbed in $\mathrm{g}$ - $\mathrm{U} / \mathrm{kg}$-ads, $\Gamma_{n}$, as a function of harvest time, $t$, and number of elution cycles, $n$ considering the AF1 adsorbent with $\beta_{\max }=$ $5.421 \mathrm{~kg}-\mathrm{U} / \mathrm{t}$-ads, $K_{D}=22.6$ days, and $d=5$ (Gill et al, 2016). The value resulting from $t$ and $n$ from the study conducted by Picard et all (2014) is indicated by the red star. Parameter combinations outside of the one year timeframe are shaded. The optimal value within the limited region is shown by the red circle.

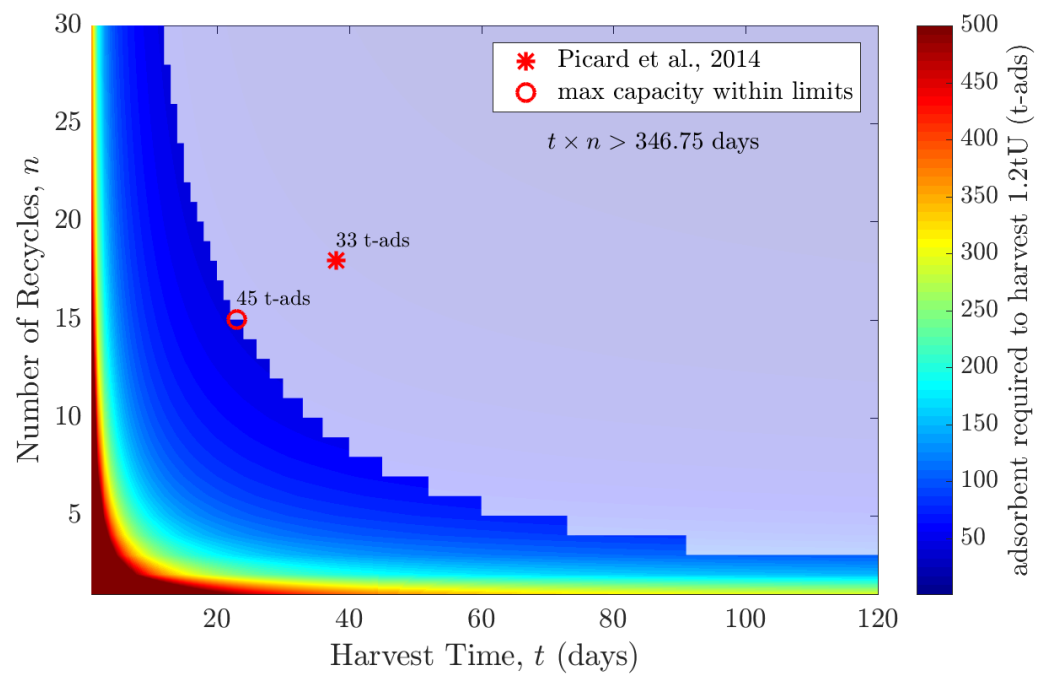

Figure 2-5: As in figure 2-4 but for the amount of adsorbent required in $\mathrm{kg}$ to harvest $1.2 \mathrm{t}-\mathrm{U}$ annually.

[2-5. As can be seen from the Figures [2-4 and $[2-5$, the parameters chosen by (Picard et all, 2014) would not fit within the one year time limit. The optimal values for the AF1 adsorbent within this timeframe actually occur with $t=23$ days and $n=15$ cycles, resulting in $\Gamma_{15} \sim 26 \mathrm{~kg}-\mathrm{U} / \mathrm{t}$-ads and requiring $\sim 45$ t-ads. 


\subsection{Experimentally tested adsorbent uptake}

The parameters $\beta_{\max }$ and $K_{D}$ are experimentally determined from fitting time series data for uranium uptake for various adsorbents to the one-site ligand saturation model. Detailed in this section are the results of experiments on the most prominent adsorbents developed to date and their respective experimentally determined parameters.

\subsubsection{Adsorbent Performance Characteristics}

Over the past ten years, various novel amidoxime-based polymer adsorbents have been developed at Oak Ridge National Laboratory (ORNL). These adsorbents have then been tested at Pacific Northwest National Laboratory (PNNL)'s Marine Sciences Laboratory (MSL) under natural seawater and exposure conditions. The performance of three (38H, AF1, AI8) formulations of the adsorbents are described in this section.

All three adsorbent formulations were prepared using hollow-gear-shaped, high surface area polyethylene fibers by radiation-induced graft polymerization (Kim et al., 2013b, 2014, 2013a; Das et al., 2016a,b). The different formulations of the fibers are determined by the different comonomer grafted. $38 \mathrm{H}$ refers to the adsorbent grafted with a methacrylic acid comonomer, AF1 to that grafted with itaconic acid, and AI8 with vinylphosphonic acid (Das et al, 2016a, [b).

Before seawater testing, all adsorbents were pretreated by immersion in a $2.5 \%$ $\mathrm{KOH}$ solution, a step which has been found to enhance the hydrophilicity of the adsorbents and induce swelling of the adsorbent fibers, both results that are beneficial for facilitating uranium adsorption (Omichi et al., 1986; Pan et al., 2015).

The various adsorbents were tested in one of two types of experiments. The first of which was a column flow-through experiment in which filtered and temperature controlled seawater was pumped through adsorbents that were packed between glass beads in 1 in diameter PVC columns. The second type of experiment was a flowthrough flume experiment in which temperature controlled and filtered seawater was pumped through a recirculating open channel containing adsorbent braids (Kun et al, 2016; Gill et al, 2016). Table 22 details the results of the flow-through column tests and table 2.3 details the results of the flume tests. Note that for all tests, the exposure temperature was controlled at $20 \pm 1.5^{\circ} \mathrm{C}$ and the results were normalized to a salinity of $35 \mathrm{psu}$.

Of all the formulations, AI8 has the best uranium adsorption performance, with a 56 -day adsorption capacity of $4.13 \pm 0.41 \mathrm{~g} \mathrm{U} / \mathrm{kg}$ adsorbent and a modeled saturation capacity of $6.86 \pm 0.68 \mathrm{~g} \mathrm{U} / \mathrm{kg}$ adsorbent, based on flume tests (Gill et al, 2016).

\subsubsection{Temperature Dependence}

Sekiguchi et al. (1594) concluded that there is a noticeable effect of seawater temperature on the adsorbent capacity in harvesting uranium from seawater. They found that in general, the capacity coefficient of the amidoxime adsorbent increased with increased seawater temperature. Tamada (20)(19) also noted the seawater temperature 
increased the uranium adsorption (figure [2-6]), from two adsorbent experiments. In the first experiment, adsorbent stacks were deployed in the Mutsu area in which the seawater had a temperature of $20^{\circ} \mathrm{C}$ and in the second experiment, braided adsorbent was tested off the coast of Okinawa, where the seawater temperature was $30^{\circ} \mathrm{C}$. This $10^{\circ} \mathrm{C}$ difference enhanced the uranium adsorbent of the adsorbent stacks by 1.5 times, as shown in figure [2-6].

Through recent experiments by PNNL, the relationship between ocean temperature and the uptake of uranium by adsorbents developed by ORNL was determined (Gill et al., 2014). For this study, time series data for the uptake of the adsorbent was measured at different temperatures. Given the limited data, a linear regression

Table 2.2: One-site ligand saturation modeling of time-dependent measurements of uranium from flow-through column tests for three adsorbent formulations obtained from ORNL (Kuo et al, 2016)

\begin{tabular}{|c|c|c|c|}
\hline $\begin{array}{c}\text { ORNL } \\
\text { Adsorbent }\end{array}$ & $\begin{array}{c}\text { 56-day adsorption } \\
\text { capacity } \\
\text { (g U/kg ads) }\end{array}$ & $\begin{array}{c}\beta_{\max } \text {, saturation } \\
\text { capacity } \\
\text { (g U/kg ads) }\end{array}$ & $\begin{array}{c}K_{D} \text {, half-saturation } \\
\text { time (days) }\end{array}$ \\
\hline $38 \mathrm{H}$ & $3.37 \pm 0.26$ & $4.49 \pm 0.27$ & $18.6 \pm 3.00$ \\
\hline $\mathrm{AF} 1$ & $3.86 \pm 0.18$ & $5.43 \pm 0.19$ & $22.8 \pm 1.90$ \\
\hline $\mathrm{AI} 8$ & $3.54 \pm 0.17$ & $5.17 \pm 0.18$ & $25.8 \pm 2.14$ \\
\hline
\end{tabular}

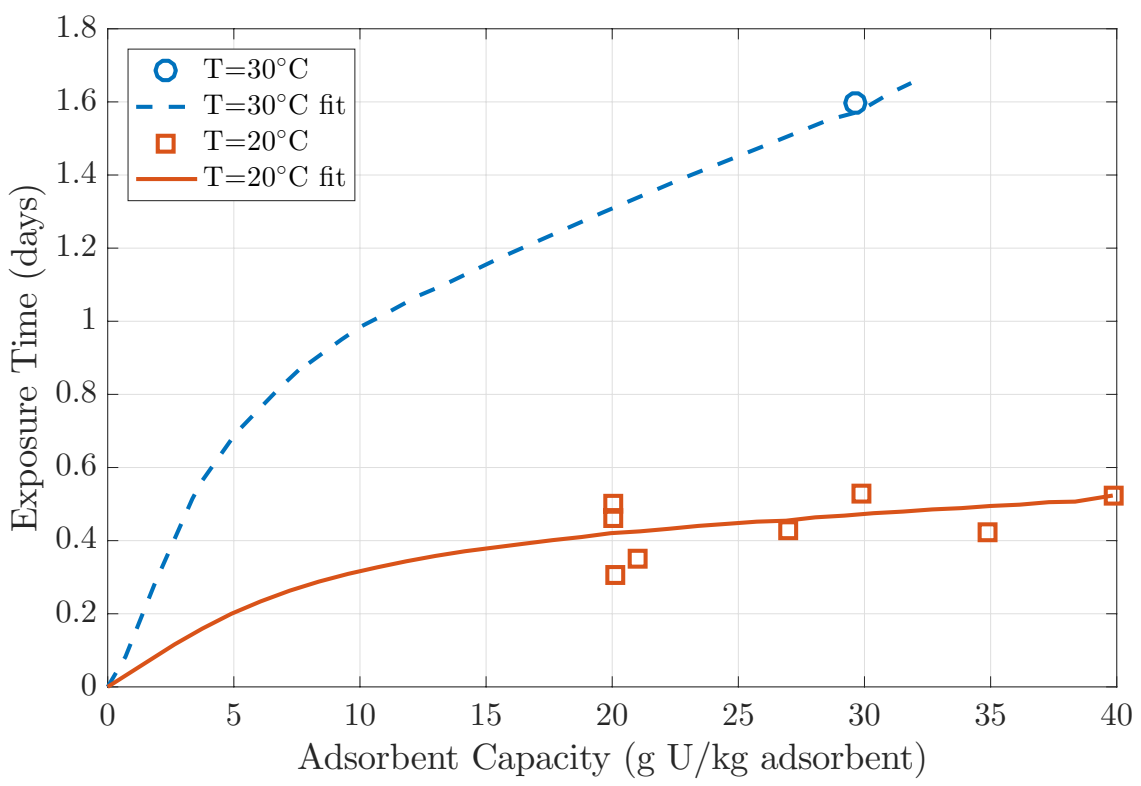

Figure 2-6: Uranium adsorbent capacity (g-U/kg-ads) as a function of soaking time in days and water temperature from Tamada (20)(1)). Data from two adsorbent experiments were used: braided adsorbent that was in $\mathrm{T}=20^{\circ} \mathrm{C}$ (red squares) and adsorbent stacks that were in $\mathrm{T}=30^{\circ} \mathrm{C}$ blue circles). The dotted blue line and solid red line represent the one-site ligand-saturation model fit to the data for the braided adsorbent and adsorbent stacks, respectively. 
Table 2.3: One-site ligand saturation modeling of time-dependent measurements of uranium from flume tests for two adsorbent formulations obtained from ORNL (Gill et all, (2016)

\begin{tabular}{|c|c|c|c|}
\hline $\begin{array}{c}\text { ORNL } \\
\text { Adsorbent }\end{array}$ & $\begin{array}{c}\text { 56-day adsorption } \\
\text { capacity } \\
\text { (g U/kg ads) }\end{array}$ & $\begin{array}{c}\beta_{\max } \text {, saturation } \\
\text { capacity } \\
\text { (g U/kg ads) }\end{array}$ & $\begin{array}{c}K_{D}, \text { half-saturation } \\
\text { time (days) }\end{array}$ \\
\hline $\mathrm{AF} 1$ & $4.03 \pm 0.12$ & $5.93 \pm 0.17$ & $26.5 \pm 1.6$ \\
\hline $\mathrm{AI} 8$ & $4.13 \pm 0.41$ & $6.86 \pm 0.68$ & $37 \pm 7.0$ \\
\hline
\end{tabular}
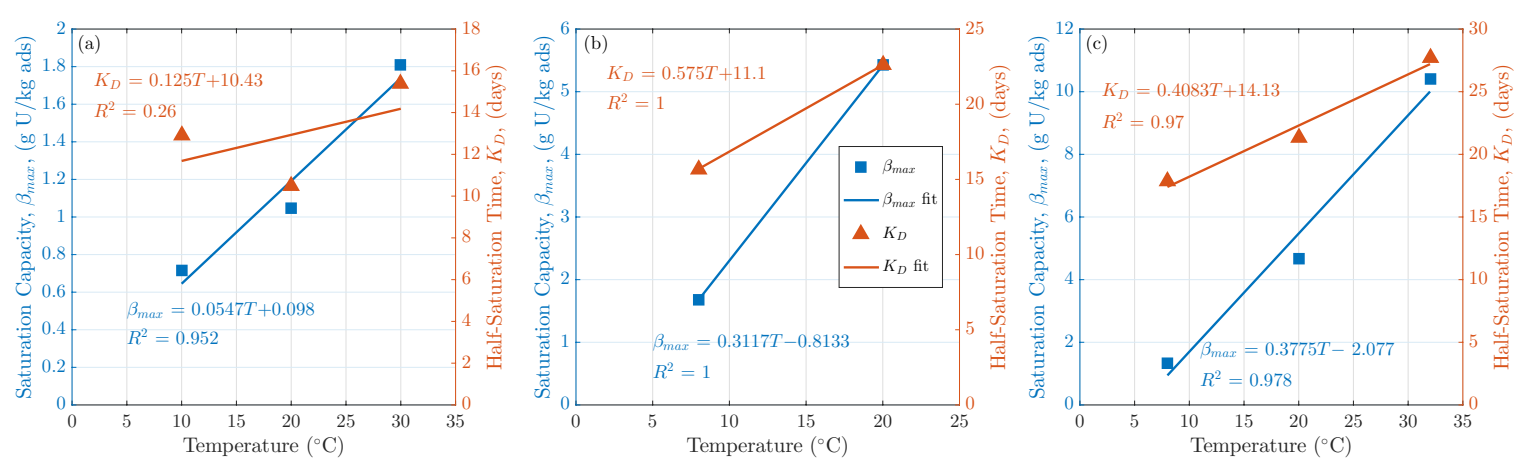

Figure 2-7: Temperature dependance of kinetic parameters $\beta_{\max }$ (blue squares) and $K_{D}$ (red triangles) for (a) $38 \mathrm{H}$, (b) AF1, and (c) AI8 ORNL adsorbents as determined by experiments by PNNL. The blue and red lines indicate the linear fit of the $\beta_{\max }$ and $K_{D}$ data sets respectively (Gill et al., 2014)

was performed on all the adsorbent types analyzed in the PNNL marine experiments (Byers, 2015 ). The results of the temperature dependence of the kinetic parameters, $\beta_{\max }$ and $K_{D}$, for the three ORNL adsorbents is shown in figure $2-7$.

In the case of the AF1 adsorbent, only two temperature experiments were utilized for the linear model fit because the data from the experiment containing the fiber at $32^{\circ}$ was not usable. The high $R^{2}$ values for the same linear regression used on the kinetic parameters for the other two adsorbents suggests that the linear regression model could be used for the case of the AF1 adsorbent as well. Furthermore, there was agreement within $5 \%$ of the the linear fit model for the AF1 adsorbent with one recoverable data point from the missing dataset, further suggesting the linear fit was reasonable (Byers, [2015).

The temperature dependent models for the coefficients $\beta_{\max }$ and $K_{D}$ can then be placed back into the one-site ligand model, (2.2), to yield the following temperature dependent relationships for uranium uptake of the $38 \mathrm{H}, \mathrm{AF} 1$, and AI8 adsorbent, respectively

$$
\begin{aligned}
C_{\text {max }, 38 H} & =\frac{(0.0547 T+0.098) t}{(0.125 T+10.43)+t}, \\
C_{\text {max }, A F 1} & =\frac{(0.3117 T-0.8133) t}{(0.575 T+11.1)+t},
\end{aligned}
$$




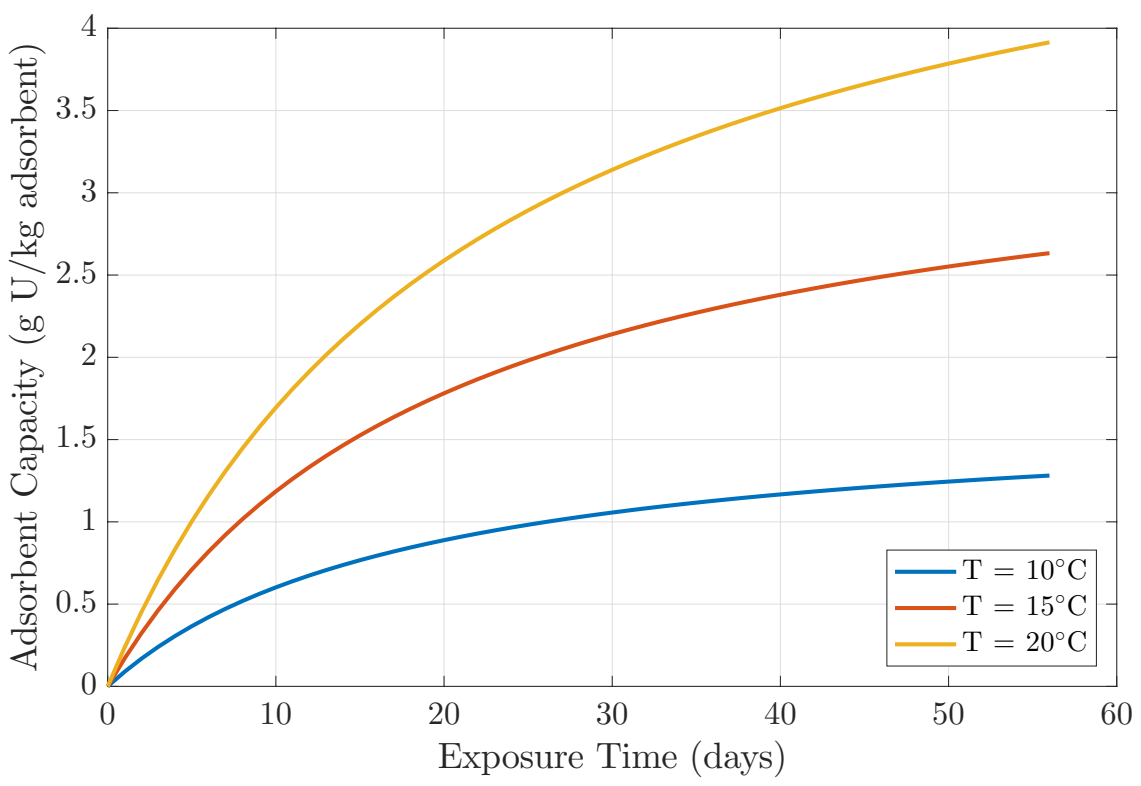

Figure 2-8: Time-dependent uptake of uranium for the AI8 adsorbent fiber as predicted by (2.15) for $\mathrm{T}=10^{\circ} \mathrm{C}$ (blue), $15^{\circ} \mathrm{C}$ (red) and $20^{\circ} \mathrm{C}$ (yellow).

$$
C_{\max , A I 8}=\frac{(0.3775 T-2.077) t}{(0.4083 T+14.13)+t}
$$

where $C_{\max }$ is the uranium uptake of the adsorbent (g-U/kg-adsorbent) and $T$ is the temperature in ${ }^{\circ} \mathrm{C}$. Figure $[2-8]$ shows the resulting time-dependent uptake for the AI8 adsorbent for $\mathrm{T}=10^{\circ} \mathrm{C}, 15^{\circ} \mathrm{C}$ and $20^{\circ} \mathrm{C}$ as predicted by (2.15). As can be seen from the figure, just a $5^{\circ}$ difference in temperature results in an over $50 \%$ increase in the uranium uptake. This is critical because, with the exception of the surface mixed layer, most of the seawater in the ocean is at temperatures ranging from $\approx 5-10^{\circ} \mathrm{C}$, suggesting that uptake of uranium in an ocean setting for all these adsorbents will likely be much less than the adsorbent capacities determined in the lab experiments presented here.

\subsubsection{Biofouling Effects}

Biofouling is the accumulation of organisms on wetted surfaces that is generally characterized as a four-step process (Aharzila et al, 11999; Lejars et al., 2012). In the first stage, which occurs within 5-10 seconds of immersion, surfaces are rapidly coated with an organic conditioning film (Callow and Fletcher, 1994). After this, single bacterial cells and diatoms begin to settle, adhere and colonize the surface (Walt et al., 1.985). Microbial films develop in the third stage, creating rough surfaces that trap more particles and organisms including larval forms of macroorganisms such as barnacles. In the fourth and final stage, overgrowth of macroorganisms, such as barnacles, occur on the fouled surface (Chambers et al., 20)(1); de Messano et al., 20().9).

The design features of the adsorbents that are intended to maximize uranium 


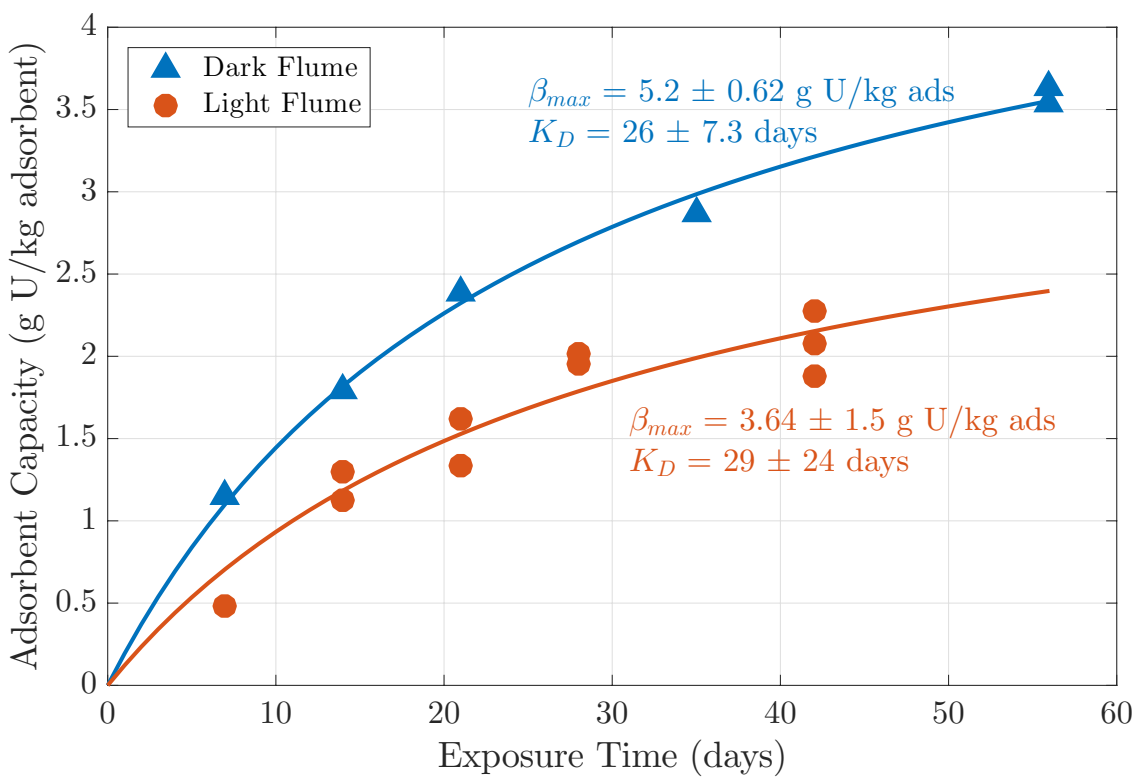

Figure 2-9: Time-dependent uptake of uranium for the AI8 adsorbent fiber in a recirculating seawater flume with no light exposure (blue triangles) and with light exposure (red circles). The blue and red lines indicate the one-site ligand model fit by Equation (2.2), respectively (Park et al, 2016). The coefficients of the model, $\beta_{\max }$ and $K_{D}$ are presented with their $95 \%$ confidence intervals. Adapted from Park et all (2016).

uptake, such as large porous or textured surface areas with micro or nanoscaled features, may also enhance biofouling (Hills and Thomason, 11998; Berntsson et al, 201010; Callow et al, 20102; Howell and Behrends, [2006). For this reason, it was hypothesized that, in the presence of biofouling, the increase in biomass on the adsorbent would decrease uranium uptake by limiting the accessibility of the ligands to ocean water. It was also hypothesized that the uranium recovery process would be decreased by dilution of the extraction solution and/or restricting its access to the ligands (Park et all, 2016).

Experiments were developed by PNNL to quantify the potential impact of fouling on uranium uptake (Park et al, [2016). In particular, light has been shown to be a primary driver of algae and microorganism growth and hence biofouling. Therefore, a set of time series flume experiments examined the uranium uptake of the AI8 adsorbent in a flume with no light exposure and a flume with full light exposure. In these experiments, ambient seawater was filtered through a $150 \mu \mathrm{m}$ filter before entering the flume. The $150 \mu \mathrm{m}$ filter was chosen because it allowed for the free passage of most phytoplankton species that contribute to biofouling, but removed larger species. The light intensity of the flume exposed to light was approximately 2,700 lumens $/ \mathrm{ft}^{2}$, which is a small fraction of the light intensity of a bright afternoon $\left(\sim 108,000\right.$ lumens $\left./ \mathrm{ft}^{2}\right)$. The results of the uranium uptake for the AI8 adsorbents in both of these flumes is shown in figure 2-9 (Park et al, 2016).

The biofouling had little effect on the rate of uranium uptake by the adsorbent, 
as seen by the fact that the half-saturation time, $K_{D}$, remains nearly unchanged. However, the biofouling decreased the fiber's overall uptake and capacity, given the significant difference in the predicted saturation capacity, $\beta_{\text {max }}$. This effect was seen almost immediately. In this study, the saturation capacity of the fouled fibers in the light flume was reduced by approximately $30 \%$ as compared to the unfouled fibers in the dark flume.

Furthermore, the degree of biofouling depends on temperature. In general, the adsorbent in warmer waters will likely experience a greater amount of biofouling. White et al. (1097) present the following formula relating the heterotrophic bacterial specific growth rate, $G\left(\right.$ day $\left.^{-1}\right)$, and the ocean temperature, $T\left({ }^{\circ} \mathrm{C}\right)$ :

$$
\log (G)=-1.54+(0.052 \pm 0.05) T \text {. }
$$

At the same time, uranium complexation with amidoxime favors warmer waters, driving competing feedback between these two factors. Furthermore, while the photic zone, which is the location of most biofouling activity, is near the ocean surface, where the waters are also warmest. Moving the fibers into deeper waters will likely reduce the amount of light penetration and hence biofouling activity which would increase uranium uptake; it will also place them in colder waters, thereby acting to reduce the uranium uptake.

\subsubsection{Influence of Current Velocity}

Experiments have also been conducted on the ORNL developed adsorbents to quantify the influence of current velocity on uranium uptake. Specifically, Ladshaw et al. (2017) subjected the AF1 adsorbent to linear velocities ranging from $0.48-8.24 \mathrm{~cm} / \mathrm{s}$ in a recirculating flume configuration to determine the affect the linear velocity had on the adsorbent's uptake of uranium.

To describe adequately the experimental data, an adsorption model was developed by Ladshaw et al. (2017) that accounted for the reaction and mass-transfer kinetics. In particular, the concentration of uranium in the adsorption domain as a function of the adsorption reaction and mass transfer from bulk solution was taken to be

$$
\epsilon \frac{\partial c}{\partial t}=\epsilon K\left(C_{b}-c\right)-\rho_{f}(1-\epsilon) \frac{\partial q}{\partial t}
$$

where $\epsilon$ is the average void of the domain, $c(\mathrm{~mol} / \mathrm{L})$ is concentration distribution of uranium inside the adsorption domain, $K(1 / \mathrm{s})$ is a parameter quantifying masstransfer effects, $C_{b}(\mathrm{~mol} / \mathrm{L})$ is the average uranium concentration of seawater, $\rho_{f}$ $(\mathrm{kg} / \mathrm{L})$ is the fiber's density, and $q(\mathrm{~mol} / \mathrm{kg})$ is the number of available ligand sites already in use. In this equation, $K$, the mass-transfer parameter, represents the effects of interparticle and interphase transport, and should theoretically vary with linear velocity (Tien, 1994).

In particular, the flume experiments showed extreme differences in adsorption

capacity as a function of linear velocity, as seen in figure $2-10$. This suggests that the external mass-transfer resistance had not been successfully removed (Ladshaw et al, 
2017).

Further investigation of the optimal mass-transfer coefficient, $K$, as described in figure [-1], determines a clear relationship between the optimal values of the mass-transfer coefficient and the linear velocities in the flume experiments. For the velocities between $0.48 \mathrm{~cm} / \mathrm{s}$ and $5.52 \mathrm{~cm} / \mathrm{s}$, the results indicate there is a strong, positive relationship between linear velocity and mass transfer, as expected. The highest linear velocity of $8.24 \mathrm{~cm} / \mathrm{s}$ deviates downward from the trend, suggesting the mass-transfer coefficient is reaching a plateau where it will no longer increase with increasing linear velocity (Ladshaw et al, 2017).

Although these recent studies greatly enhance the understanding of the adsorbent kinetics at higher flow rates, $8.24 \mathrm{~cm} / \mathrm{s}$ is still a fairly slow current speed for the open ocean. Currents of $10-15 \mathrm{~cm} / \mathrm{s}$ are much more likely, and even up to $1-2 \mathrm{~m} / \mathrm{s}$ in extremely strong boundary currents such as the Gulf Stream. Thus, it is likely the case that for all ocean deployments that the linear velocity is not the rate limiting factor in the uranium uptake of the adsorbent. This result has also been confirmed by tests done off the dock of the Woods Hole Oceanographic Institute (Buesseler, 2017).

\subsubsection{Potential Adsorbent Loss}

Though not experimentally tested to-date, there is concern that all adsorbent fibers detailed in this chapter will undergo loss when placed in an ocean setting. This loss could be physical due to the ocean current velocities which are much greater than those investigated in the study by Ladshaw et al. (2017). Animal consumption of the adsorbent fibers could also occur. As more biofouling accumulates on the adsorbent

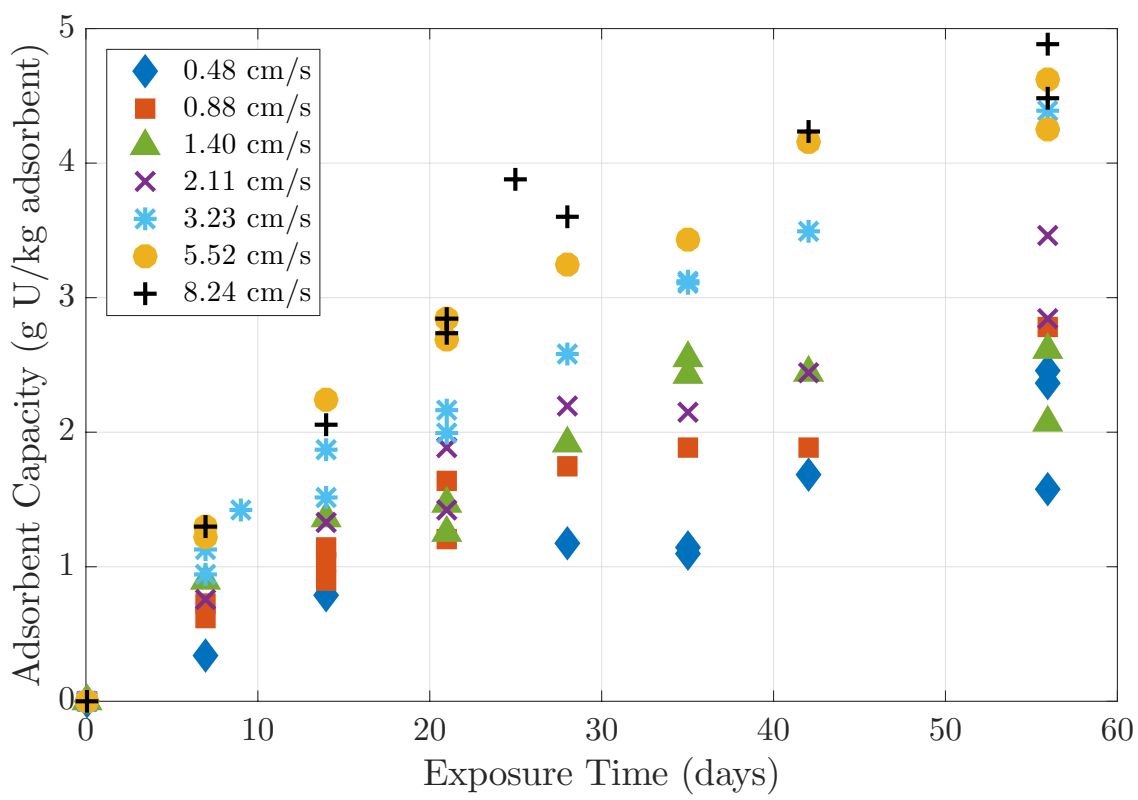

Figure 2-10: Time-dependent uptake of uranium for the AF1 adsorbent fiber in recirculating seawater flumes of various linear velocities. Adapted from Ladshaw et al. (2017). 


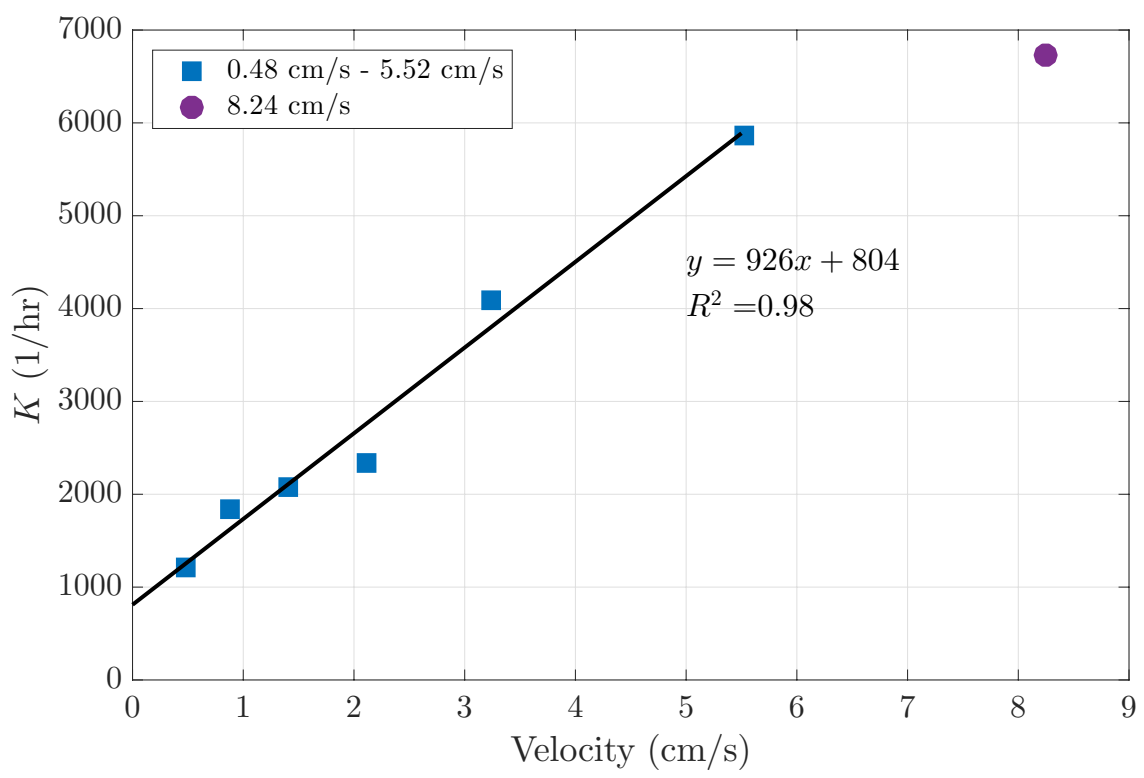

Figure 2-11: Relationship between the optimal values of the mass-transfer coefficient, $K$, and the linear velocities in the flume experiments. Adapted from Ladshaw et al. (2017).

overtime, larger marine animals will likely prey on these organisms, inadvertently eating some of the adsorbent fibers to which these organisms are attached.

\subsection{Chapter Summary}

In this chapter, the composition of uranium in seawater and its concentration relative to other metal ions was described. Additionally, the binding of seawater uranium to amidoxime was presented. Moreover, the characteristics of various adsorbents developed by ORNL to extract uranium from seawater were described. Specifically, experiments on these adsorbents have shown that the adsorbent uptake increases with increasing temperature and increases with increasing velocity, up to a certain value (after which it reaches a plateau). However, the experiments also showed that biofouling negatively affects the adsorbent uptake of uranium which can be reduced by up to $30 \%$. Biofouling also favors warmer temperatures and currently no studies have examined the competing effects of increased temperature and hence increased biofouling on the adsorbent capacity. 


\section{Chapter 3}

\section{Shell Enclosure Strategy}

This chapter describes a new shell enclosure strategy developed for use with the adsorbent fiber which allows for the decoupling the of material's functional requirements of strength and adsorption.

\subsection{Motivation}

In general, the amidoxime-based polymer adsorbents presented in the literature have inherently low tensile strength properties. For these reasons, the ligands are often grafted onto a polyethylene trunk fiber that can provide high tensile strength. Research has shown that while the tensile strength of the trunk ultra-high molecular weight polyethylene (UHMWPE) fiber may be more than 3.0 GPa, after $\gamma$-irradiation to induce grafting of the amidoxime ligands, this strength is decreased drastically to $1.3 \mathrm{GPa}$ (Xing et al., 2013).

This decreased strength after irradiation is due to two factors. First, $\gamma$-irradiation causes the degradation of the UHMWPE molecular chain, thereby leading to a decrease in the molecular weight of polyethylene. Second, the radiation cross-linking of the UHMWPE fiber restricts the mobility of molecular chains of polyethylene, and causes nonuniformity of stress in the fiber (Yamanaka et al., 2006; Zhao et al, [2010).

Additionally, the decrease in strength after $\gamma$-irradiation was found to be dependent on the adsorbed dose. A higher radiation dose, the greater the impairment to the mechanical properties of the fibers which influences the lifetime and recyclability of the adsorbents (Hu et al., 2016). Thus, a method of decoupling the chemical and mechanical properties of the adsorbent polymer would allow for the independent optimization of both and likely lead to adsorbents with much higher adsorbent capacity.

\subsection{Theory}

A two-part system is developed to decouple the mechanical and chemical needs of an adsorbent for seawater harvesting of uranium. In the system, a hard permeable outer 
(a)

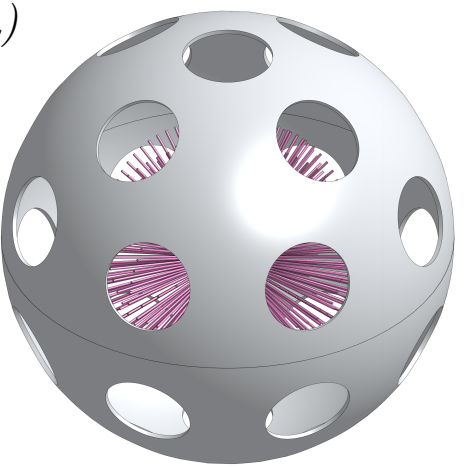

(b)

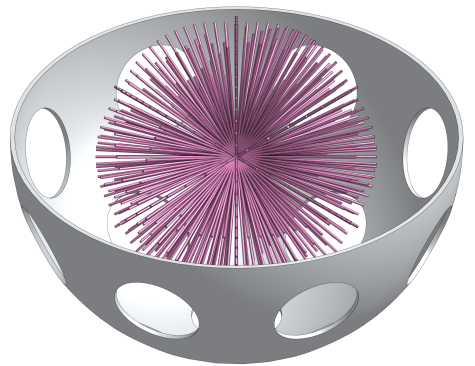

Figure 3-1: Initial adsorbent concept with decoupling of mechanical and chemical requirements. Soft, inner adsorbent sphere is encased in tough, outer protective sphere. Outer sphere features holes to allow adequate seawater to adsorbent interior.

shell with sufficient mechanical strength and durability for use in an offshore environment and chemical resilience against elution treatments serves as the protective element for uranium adsorbent material with high adsorbent capacity in its interior. The chemistry of the inner material can thus be optimized for higher adsorbent capacities, while the mechanical properties required of the system are achieved by the hard permeable outer structural shell, resulting in a system that is more cost effective for implementation.

Figure $3-1$ depicts one shell design in which a spherical hard permeable outer shell encloses uranium adsorbing material inside. The uranium adsorbing material is wound into a ball with filaments extending radially outward from the center core (referred to as the filament ball). The holes in the outer shell are sized so that seawater may continually pass relatively easily to the interior of the shell where the uranium adsorbing material is housed, while maintaining sufficient mechanical strength to withstand the forces of the offshore system that must move the units through the water and collect and disperse them. The outer shell is preferably made of plastic, such as polyethylene, so that it can have high chemical resilience and therefore can withstand multiple elution cycles as required by the offshore seawater uranium harvesting system. By making the outer shell out of two distinct upper and lower hemispheres, it can be disassembled and reassembled for the easily placement and replacement of the inner uranium adsorbing material and can be reused many times for multiple changes of adsorbents. 


\subsection{Adsorbent Interior}

The adsorbent interior is modeled by two main parts: (1) a filament core of diameter $d_{f c}$, and (2) a series of fibers of length $l_{f}$ radiating outward from the spherical surface of the filament core. This is done because it is assumed that there is some critical region in which the adsorbent fibers will be so tightly wound that the water will not be able to reach them and hence should not be considered when determining the uranium uptake of the filament ball. The overall shell diameter is given by $d_{s}$ and the distance between the edge of a filament fiber and the edge of the enclosing shell is given by $d_{f, s}$. A schematic of this model is shown in figure $3-2$.

The amount of adsorbent required by the system is determined by

$$
m_{a d s}=\frac{U_{r e q}}{\Gamma_{n}}
$$

where $U_{r e q}$ is the amount of uranium to be harvested annually and $\Gamma_{n}$ is determined by (2.T2) for a specified number of elution cycles, $n$, and harvest time, $t$.

The length of the system, $l_{\text {sys }}$ is defined to be the total length of all shells if they were placed to end to end such that the total number of shells is defined by

$$
N_{s}=\frac{l_{\text {sys }}}{d_{s}} .
$$

This is then used to determine the linear distribution of adsorbent as

$$
l_{a d s}=\frac{m_{a d s}}{l_{\text {sys }}} .
$$

The amount of adsorbent required to be in each shell is then determined by

$$
m_{a d s, s}=\frac{l_{a d s}}{d_{s}}
$$

and the total length of fiber required per shell is

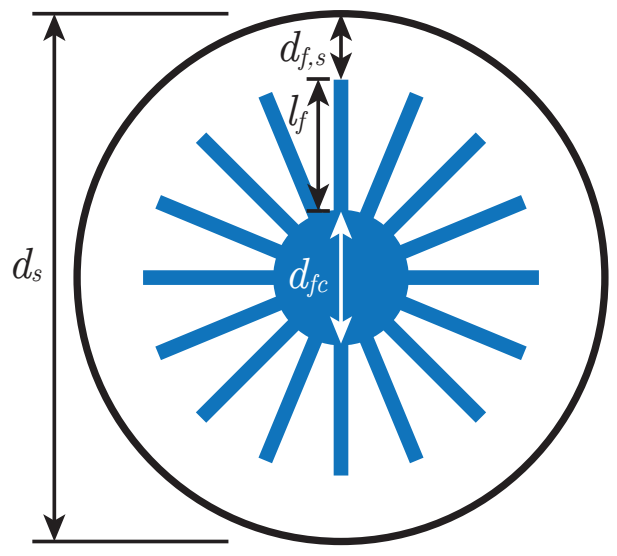

Figure 3-2: Schematic of filament ball of uranium adsorbing fibers and shell enclosure model. 


$$
l_{r e q, \text { shell }}=\frac{m_{a d s, s}}{\rho_{f} \pi\left(\frac{d_{f}}{2}\right)^{2}},
$$

where $\rho_{f}$ is the density of the adsorbent fiber and $d_{f}$ is the diameter of the adsorbent fiber (both properties taken from Gill et all (2016)) .

Next, the adsorbent limit per shell is computed and compared to $l_{\text {req,shell }}$ to determine if a specific design is feasible. First, the diameter of the filament core, $d_{f c}$ is set to be some fraction, $\gamma$, of the full shell diameter,

$$
d_{f c}=\gamma d_{s} .
$$

Then the surface area of the filament core, (i.e. the total area over which fibers are considered to be attached and emanating outward), is

$$
s_{f c}=4 \pi\left(\frac{d_{f c}}{2}\right)^{2} .
$$

The distance between the edge of a filament and the shell enclosure, $d_{f, s}$ is also selected to be some fraction, $\lambda$, of the full shell diameter,

$$
d_{f, s}=\lambda d_{s}
$$

The length of the fiber is then

$$
l_{f}=\frac{d_{s}}{2}-\frac{d_{f c}}{2}-d_{f, s} .
$$

The cross-sectional area of the fiber can be determined from its diameter as

$$
a_{f}=\pi\left(\frac{d_{f}}{2}\right)^{2} .
$$

Then the effective area required per fiber for attachment to the filament core is given by

$$
a_{f, e}=\frac{a_{f}}{\eta}
$$

where $\eta$ is the packing density of the fibers on the sphere. According to Conway and Sloane (1993), the close-packing of equal spheres has a maximum packing density of

$$
\eta_{\max }=\frac{\pi}{3 \sqrt{2}} \approx 0.74048
$$

With this in mind, $\eta$ is taken to be 0.70 . The total number of adsorbent fibers that can be packed on the filament core is then

$$
N_{f, c}=\frac{s_{f c}}{a_{e, f}}
$$

and the total length of fibers in the shell is then 


$$
l_{\text {shell }}=N_{f, c} l_{f} .
$$

If $l_{\text {shell }}<l_{\text {req,shell }}$, the design is considered feasible. Additionally, an efficiency factor, or an e-factor, can be determined for the design by

$$
\text { e-factor }=\frac{l_{\text {shell }}}{l_{\text {req }, \text { shell }}} .
$$

This factor determines how much extra adsorbent is being wound into each shell than is required. The closer to one, the more optimum of a design.

The feasibility of the uranium adsorbent material to be wound into a filament ball is detailed in figure 3 . Lines 1-4 determine the amount of adsorbent required by the system, lines 5-9 calculate the adsorbent required per ball, lines 10-21 compute the limit of how much adsorbent can be incorporated in each ball, and line 22 indicates the feasibility of the overall design. Yellow boxes indicate adsorbent specific values, and green boxes indicate tunable parameters of the mechanical system. The offshore uranium harvesting system is designed with the requirement of harvesting enough uranium to power a 5-MW nuclear reactor annually, approximately 1.2 metric tons. The rationale behind this design decision is that, if the uranium harvester was paired with a 5-MW offshore wind turbine, the entire system could harvest twice as much energy per square meter of ocean. In this example, the AF1 adsorbent is considered, where properties of $\beta_{\max }$ and $K_{D}$ were determined from the temperature dependencies described by Byers (2015). This combined with a temperature of $T=20^{\circ} \mathrm{C}$, a harvest time of $t=23$ days, $n=15$ reuses, determines the amount of adsorbent required by the system in line 1 . The density of the fiber and the adsorbent fiber diameter were taken from Gill et al. (2016). The results, with extremely conservative estimates of various mechanical parameters, prove the concept of an adsorbent filament ball to be mechanically feasible.

\subsection{Shell Enclosure}

Given the feasibility of an adsorbent filament ball, various shell enclosures were investigated for their structural strength and feasibility for use with the inner adsorbent filament ball. A strength comparison was performed of an dodecahedron, octahedron, and cube shell with circular holes in the center of each face. The geometry of the three shell types was adjusted so that they all had a maximum width of $0.5 \mathrm{~m}$. All were submitted to a vertical distributed load of $3 \mathrm{kN}$ as applied to the rim of the top face, with the bottom face full constrained. Each shell was assumed to be made of high density polyethylene (HDPE) which has yield stress of approximately $26 \mathrm{MPa}$. For each shell, the hole diameters were adjusted for each face so that the ratio of hole to solid area for each model was the same. Finally, the thickness of each structure was adjusted such that the mass of all three shells was the same. Figure 3-3 depicts the von Mises stress distribution for each of the three shells.

In addition to the von Mises stress, the factor of safety for each shell enclosure 
Table 3.1: Feasibility calculations for adsorbent filament ball.

\begin{tabular}{|c|l|l|r|}
\hline Line \# & \multicolumn{1}{|c|}{ Offshore uranium harvesting system adsorbent requirement } \\
\hline 1 & amount of adsorbent required $(\mathrm{kg})$ & $m_{\text {ads }}$ & 45,431 \\
\hline 2 & length of system $(\mathrm{m})$ & $l_{\text {sys }}$ & 6000 \\
\hline 3 & linear distribution of adsorbent $(\mathrm{kg} / \mathrm{m})$ & $l_{\text {ads }}$ & 7.57 \\
\hline 4 & total number of shells & $N_{s}$ & 12000 \\
\hline
\end{tabular}

\begin{tabular}{|c|c|c|c|}
\hline & \multicolumn{3}{|c|}{ Adsorbent required per shell } \\
\hline 5 & density $\left(\mathrm{kg} / \mathrm{m}^{3}\right)$ & $\rho_{f}$ & 950 \\
\hline 6 & adsorbent fiber diameter $(\mathrm{m})$ & $d_{f}$ & 0.001 \\
\hline 7 & diameter of a outer shell (m) & $d_{s}$ & 0.5 \\
\hline 8 & absorbent per ball (kg) & $m_{a d s, s}$ & 3.79 \\
\hline 9 & required length of fiber in ball $(\mathrm{m})$ & $l_{\text {req,shell }}$ & 5074 \\
\hline
\end{tabular}

\begin{tabular}{|c|c|c|c|}
\hline & \multicolumn{3}{|l|}{ Adsorbent limit per shell } \\
\hline 10 & filament ball core diameter to outer shell diameter (\%) & $\gamma$ & 25 \\
\hline 11 & filament ball core diameter $(\mathrm{m})$ & $d_{f c}$ & 0.125 \\
\hline 12 & distance between filament ball core and outer shell $(\mathrm{m})$ & & 0.1875 \\
\hline 13 & surface area of filament ball core $\left(\mathrm{m}^{2}\right)$ & $s_{f c}$ & 0.049087 \\
\hline 14 & distance between end of fiber length and shell (\%) & $\lambda$ & 12.5 \\
\hline 15 & distance between edge of filaments to outer shell (m) & $d_{f, s}$ & 0.0625 \\
\hline 16 & length of fiber $(\mathrm{m})$ & $l_{f}$ & 0.1625 \\
\hline 17 & cross-sectional area of adsorbent fiber $\left(\mathrm{m}^{2}\right)$ & $a_{f}$ & $7.85 \mathrm{E}-07$ \\
\hline 18 & packing density (\%) & $\eta$ & 70 \\
\hline 19 & effective area required per fiber base for attachment $\left(\mathrm{m}^{2}\right)$ & $a_{f, e}$ & $1.12 \mathrm{E}-06$ \\
\hline 20 & number of adsorbent fibers & $N_{f, c}$ & 43750 \\
\hline 21 & total length of adsorbent fibers $(\mathrm{m})$ & $l_{\text {shell }}$ & 5468.75 \\
\hline
\end{tabular}

\begin{tabular}{|c|c|c|}
\hline & \multicolumn{2}{|c|}{ Design feasibility } \\
\hline 22 & feasible design? $\left(l_{\text {shell }} \leq l_{\text {req,shell }}\right)$ & YES \\
\hline 23 & e-factor $\left(l_{\text {shell }} / l_{\text {req,shell }}\right)$ & 1.08 \\
\hline
\end{tabular}

geometry was analyzed. The shell enclosure will start to fail if a new load is applied equal to the initial $3 \mathrm{kN}$ load multiplied by the resulting factor of safety. The results of this analysis are shown in figure $3-4$. As can be seen from the figure, the cube, with the smallest number of faces, has the highest factor of safety, whereas the more spherical-like shells, such as the dodecahedron, have a much lower factor of safety and the sphere shell enclosures exhibit the lowest factor of safety. Additionally, the factor of safety decreases nonlinearly as the number of faces increases.

In addition to the structure of the shell, the geometry of the holes in the shell may also be varied. The holes must be large enough so as to allow adequate seawater flow to the enclosed adsorbent without greatly affecting the structural strength of the shell. The effect of varying hole geometries on the overall strength of the shell was 
(a)

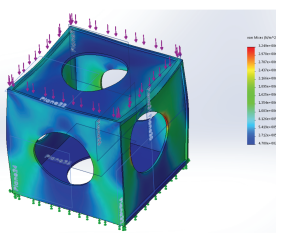

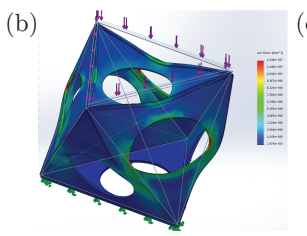
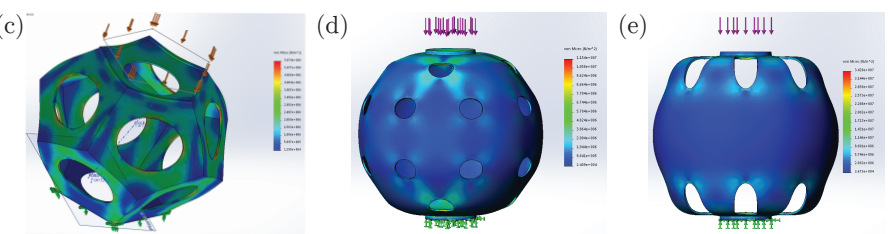

Figure 3-3: von Mises stress results for vertical loading of a (a) cube, (b) octahedron, (c) dodecahedron, (d) sphere with circular holes and (e) sphere with slotted holes shell enclosure.

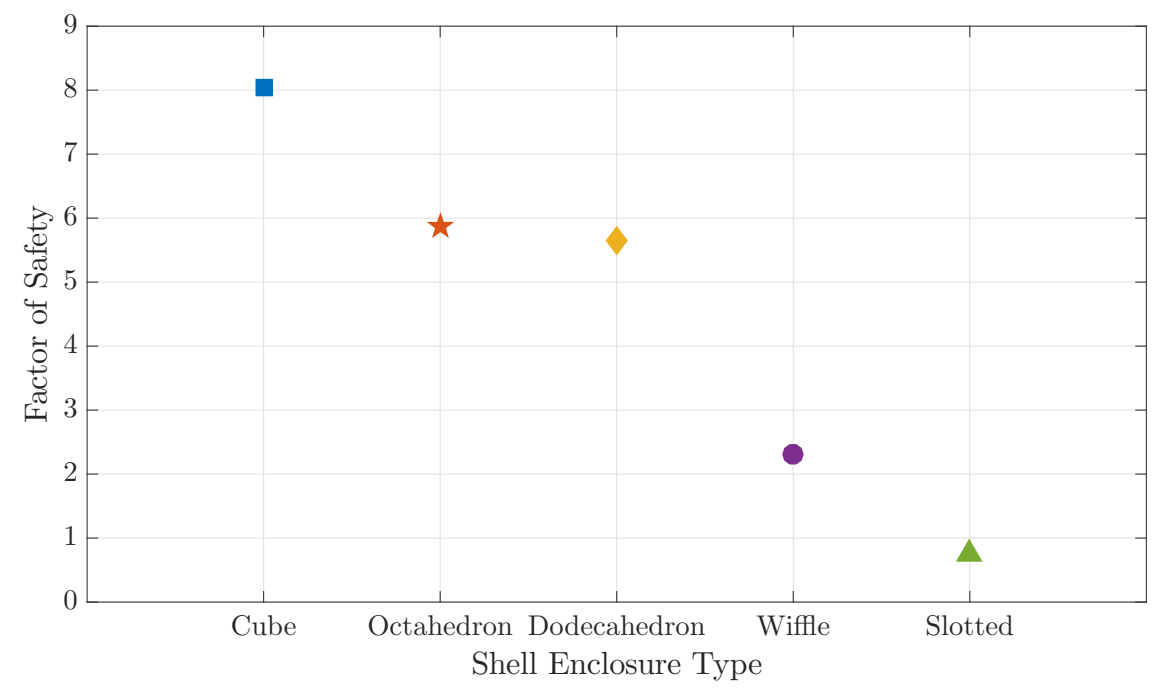

Figure 3-4: Factor of safety as a function of number of faces for the cube, octahedron, dodecahedron, sphere with circular holes and sphere with slotted holes shell enclosures.

investigated for the cube shell enclosure, given that it had the highest factor of safety. A varied set of four hole geometries was implemented, depicted in figure 3-5. As in the case of varying shell structures, the factor of safety was determined for each of the four types of hole geometries on the cube shell enclosure, with the total hole area kept constant for each model: the results are shown in figure [3-6. As can be seen from the figure, the factor of safety for the standard rectangular slits (shown in figure [3-5]) was significantly lower than for the other hole geometries. In this model, both horizontal and vertical slits were used and failure was found to occur at the edge of the horizontal slits when under a vertical load. Replacing these horizontal slits by vertical slits resulted in a "Modified rectangular slits" hole geometry, which yielded the highest factor of safety of 29.

\subsection{Conclusions}

In the case of ocean deployment of an offshore system for harvesting uranium from seawater, adsorbent materials will need to withstand the harsh environment of the ocean as well as the likelihood of rough handling during transport and deployment. 
(a)

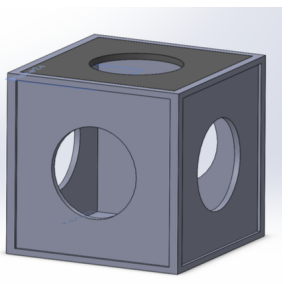

(b)

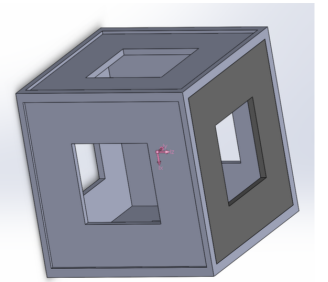

(c)

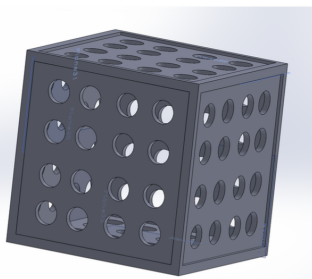

(d)

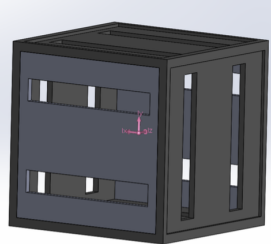

Figure 3-5: Solid models depicting cube shell enclosure with (a) large circular, (b) large square, (c) small circular, (d) rectangular slit hole geometries.

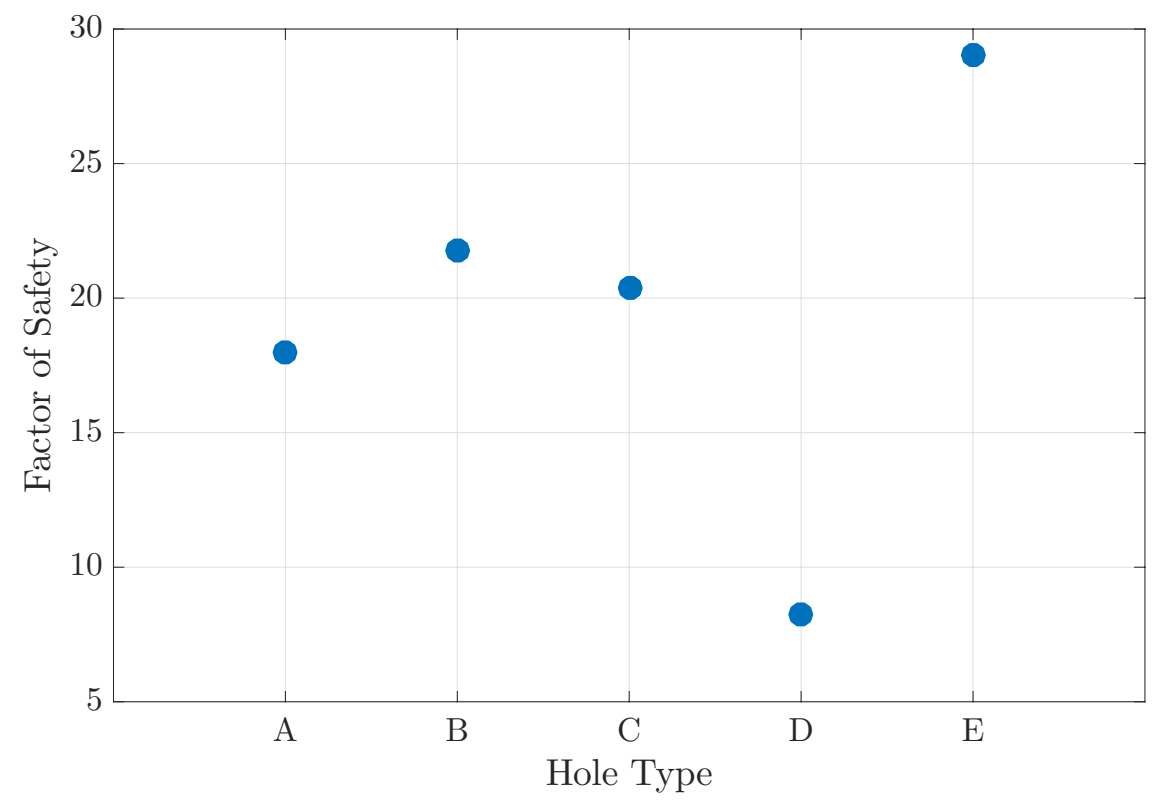

Figure 3-6: Factor of safety as a function of hole geometry for the cube shell enclosure. Hole types A-D are shown in figure $3-5$ and hole type $\mathrm{E}$ is a rotated version of hole type D.

Currently, adsorbent polymers with high tensile strength tend to have poor uranium adsorption capacity. However, the mechanical requirements of an offshore uranium harvesting system can be decoupled from the chemical requirements through the use of an exterior shell enclosure surrounding an adsorbent polymer. Furthermore, the adsorbent polymer may be wound into a ball with filaments extending radially outward from the center core. This study proved the mechanical feasibility of winding an adsorbent polymer into a filament ball to meet the annual uranium needs of a 5MW nuclear reactor.

With the structural strength of the system now provided by a shell enclosure instead of the adsorbent itself, the strength of various shell designs under vertical distributed loading was investigated. It was found that the factor of safety increased as the number of faces of the shell enclosure decreases. The cube shell likely appears to be the strongest, with a factor of safety of 8 , because its vertical walls were the most effective at resisting vertical loads. Although the cube shell was found to be the strongest, the spherical shell geometries have the benefit that the factor of safety 
is independent of the loading direction. Additionally, given that two spheres that crash into one another can easily slip past each other because they have no flat faces, a spherical shell will be the least likely of the shell designs to jam together. For these reasons, the spherical shell design was selected for the reminder of the designs of this thesis. Additional work on the effect of the hole patterns on the strength of such spherical shells is further detailed in Amanda Hamlet's Master's thesis (Hamlet, 2017).

Given that adequate seawater flow to the adsorbent interior is crucial to the total uranium adsorption of the device, the impact of four different hole geometries on the strength of the cube shell was also studied. It was determined that the vertical rectangular slits resulted in the highest factor of safety of 29 , which is likely due to the fact that the slits were aligned with the direction of the load. Given that the loads on the shells will be random, however, it is likely that the large square holes, with the second highest factor of safety of 22 , will prove to be the strongest in practice. Additional analysis needs to be conducted to determine which hole geometry is best for adequate seawater flow to the adsorbent interior. Future work should also focus on determining the distance between the filament ball and the shell enclosure for the optimal fluid flow and resulting uranium adsorption. This research is further investigated in Amanda Hamlet's Master's thesis (Hamlet, [2017). 


\section{Chapter 4}

\section{Flume Testing of Shell Enclosures}

This chapter describes the design and fabrication of an experiment to test the effect of the shell enclosures on the uptake of uranium adsorbents. This research was done in collaboration with Dr. Ken Buesseler and Jessica Drysdale at the Woods Hole Oceanographic Institution.

\subsection{Method}

Previous experiments have examined the effects of differing temperature, current speed, and degrees of biofouling on the adsorbent's ability to uptake uranium. For example, a previous study at the Pacific Northwest National Laboratory examined the effects of linear flow rates ranging from $0.48 \mathrm{~cm} / \mathrm{s}$ to $5.52 \mathrm{~cm} / \mathrm{s}$ on the adsorbent's ability to uptake uranium (Gill et al, [2015). No studies have examined the effects of a protective shell enclosure on the adsorbent's ability to uptake uranium.

\subsubsection{Shell Enclosure Design and Fabrication}

This experiments looks to investigate the affect of six shell enclosure designs on the uptake of uranium adsorbed by the fibers enclosed by the shells. One design was based on a classic wiffle ball with holes, which contained 24 holes positioned in four different tiers around the shell. Another design was based on a classic wiffle ball with slotted holes, containing eight slotted holes arranged in a circular pattern on each side of the shell. Figure 4-1 shows the six shell designs tested in this study in the order they were placed in the flume (with A being the closest to the inlet) as well as the naming convention used in the discussion. As you can see, enclosures A and B are the same design (the slotted wiffle), with the exception that they have different orientations to the flow. The same is true for enclosures $\mathrm{C}$ and $\mathrm{E}$.

Shells were fabricated in two halves and included a series of tabs and corresponding slots that allowed the two halves to be aligned each time they are connected. Each half shell was $50 \mathrm{~mm}$ (1/10th physical scale of a full scale enclosure for ocean use) in diameter and 3D printed from white acrylic. Acrylic, unlike some other plastics, will not absorb water or deform after being submerged in seawater for extended periods 


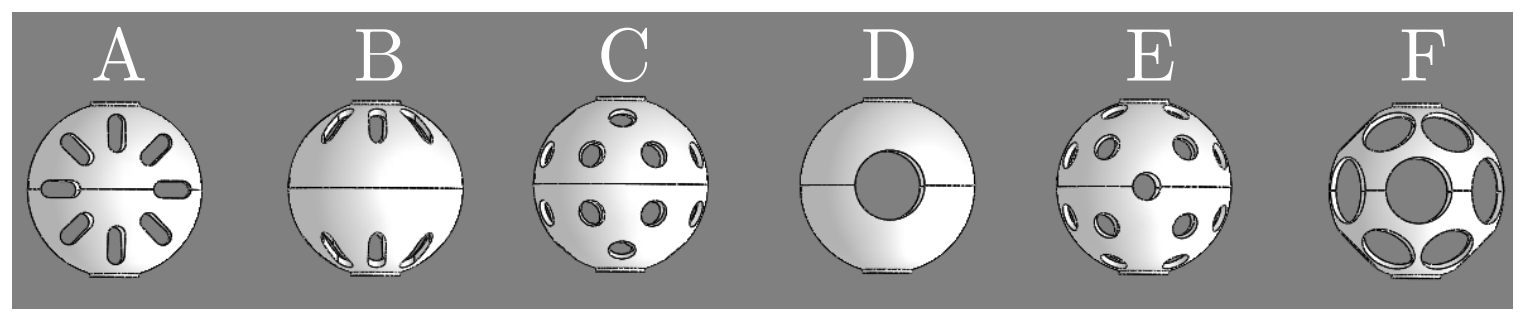

Figure 4-1: Solid models of six shell designs selected for testing.

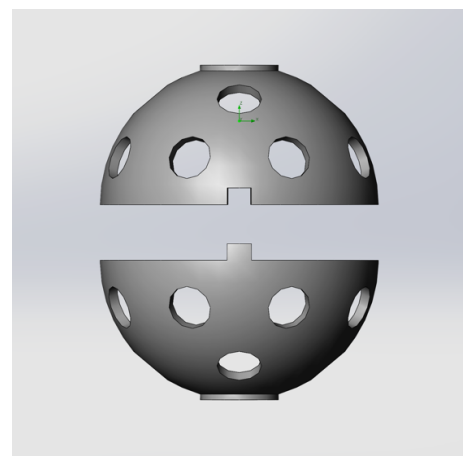

Figure 4-2: Model of a protective shell enclosure. Each shell enclosure contained multiple tabs with corresponding slots to allow alignment of the two halves.

of time. Unlike other colored acrylics, white acrylic does not contain any added dyes that could potentially leach into the seawater and affect the fibers in their uptake of uranium.

The enclosures were designed such that each shell half could be fitted to a piece of $1 / 4$ in, threaded acetal rod and secured in place with two $1 / 4$ in nylon nuts. These threaded rods were inserted into acetal blocks which were then glued to the base of a flume tank. The six shell designs were tested in a recirculating flume tank located at the Woods Hole Oceanographic Institution's Shore Lab. The tank allowed $0.45 \mu \mathrm{m}$ filtered seawater to pass through the shells continuously for 56 days.

The flume measured 72 in $\times 6$ in x 12 in and was constructed using 0.5 in acrylic. The dark colored acrylic prevented light from passing through, thereby mitigating the effects of biofouling (Park et al (2016)). Fresh filtered seawater, with temperature held at $20 \pm 1.5^{\circ} \mathrm{C}$, was fed into the system from the head tank at flow-rates up to $1.5 \mathrm{~L} / \mathrm{min}$. As fresh seawater was pumped into the flume, a 9 in stand pipe near the recirculation outlet ensured the water level in the flume remained at a constant level. An inlet and outlet allowed the seawater within the flume to be recirculated at a constant flow rate. A diagram of the flume experiment is shown in figure 4-4.

A Finnish Thompson DB8 centrifugal, nonmetalic, pump recirculated seawater in the flume at a rate of $100 \mathrm{~L} / \mathrm{min}$, corresponding to a linear flow rate in the flume of $4.8 \mathrm{~cm} / \mathrm{s}$. The flow rate was regulated using a globe valve positioned after the pump's discharge port. The volumetric flow rate was continuously monitored using an Omega FP2010-RT flow meter in line between the recirculation outlet and the pump.

To ensure that no shell enclosures were in the wake of another, the six shell 


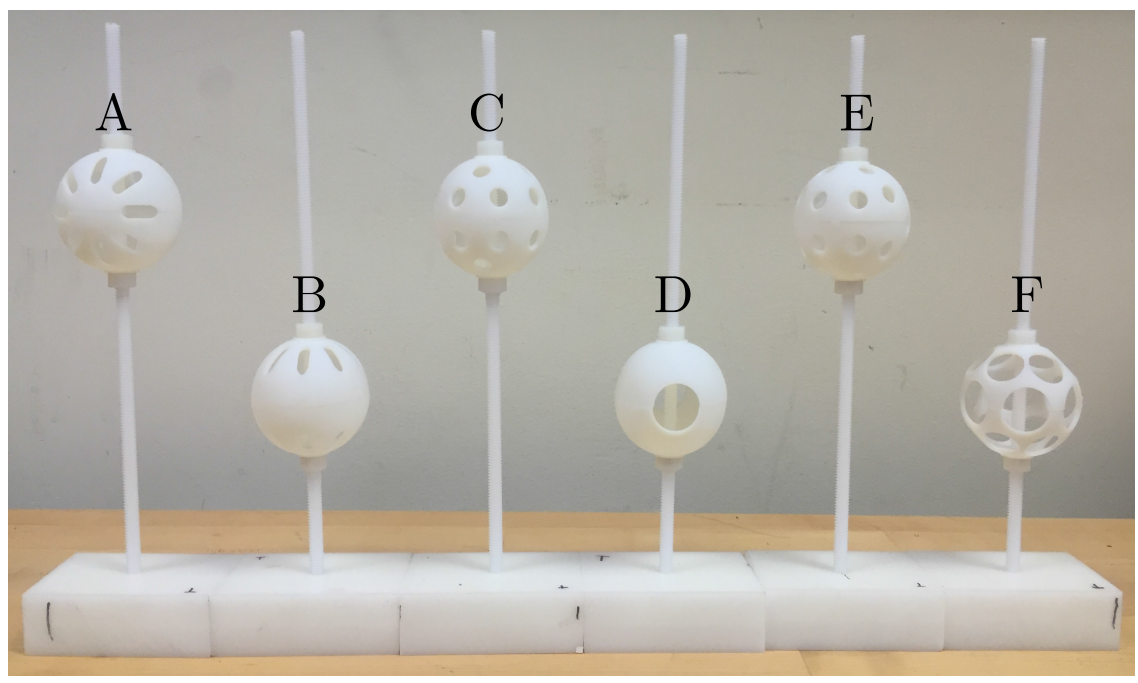

Figure 4-3: Six shell designs selected for testing. Each shell enclosure was fabricated in two halves. The upper half of each shell can be removed to allow for quick and easy sampling of the adsorbent. The outer diameter of all shell enclosures was $4 \mathrm{~cm}$.

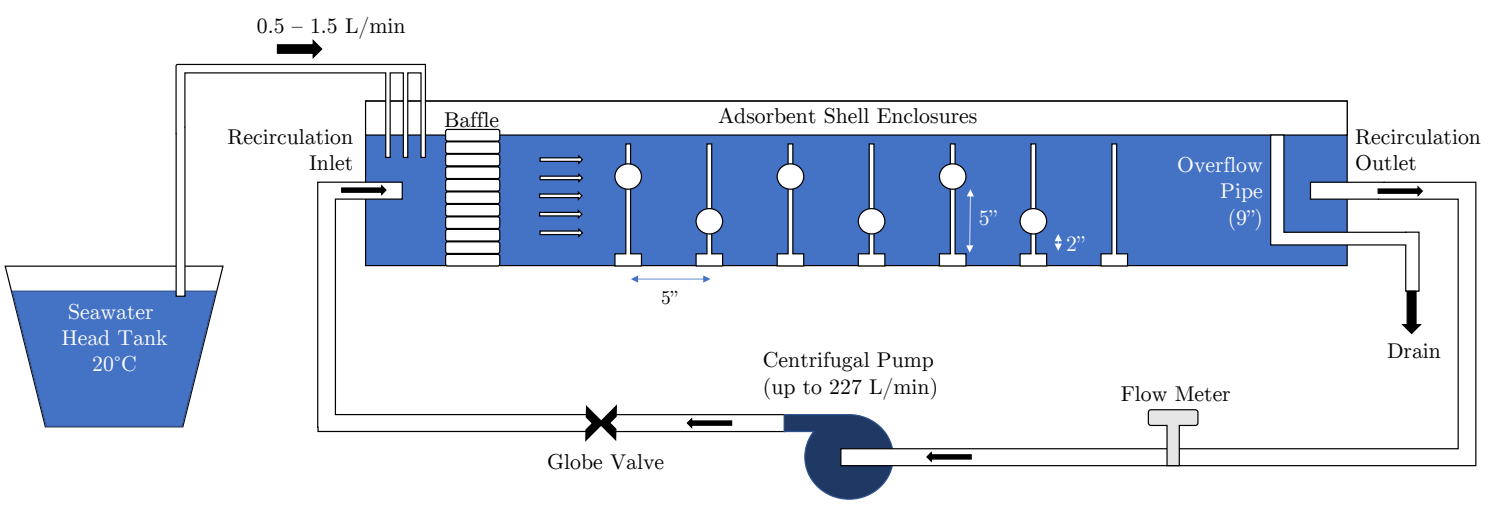

Figure 4-4: Diagram of the flume setup used to test the adsorbent enclosures. Fresh seawater is pumped in from a head tank using three tubes near the recirculation inlet. Overflow pipe ensures a constant water level of 9 in. A Finish Thompson DB8 Centrifugal Pump constantly recirculates the filtered seawater.

enclosures were staggered in their vertical placement in the flume. A seventh threaded rod and block was added to allow for an unenclosed piece of adsorbent that served as the control. A baffle, made of stacked $1 / 2$ in PVC pipe segments and installed near the recirculation inlet of the flume, minimized turbulence as the flow approached the shell enclosures.

A digital pressure meter was used to measure the pressure before the recirculation inlet and after the recirculation outlet to determine the pressure loss across the flume. The pressures were found to be 4.1 psi and 4.0 psi respectively, indicating a negligible pressure loss of 0.1 psi across the flume and confirming that the flow meter's measurement would not be affected by the pressure drop. The pump, flow meter, and all piping components were chosen such that all wetted components were made 


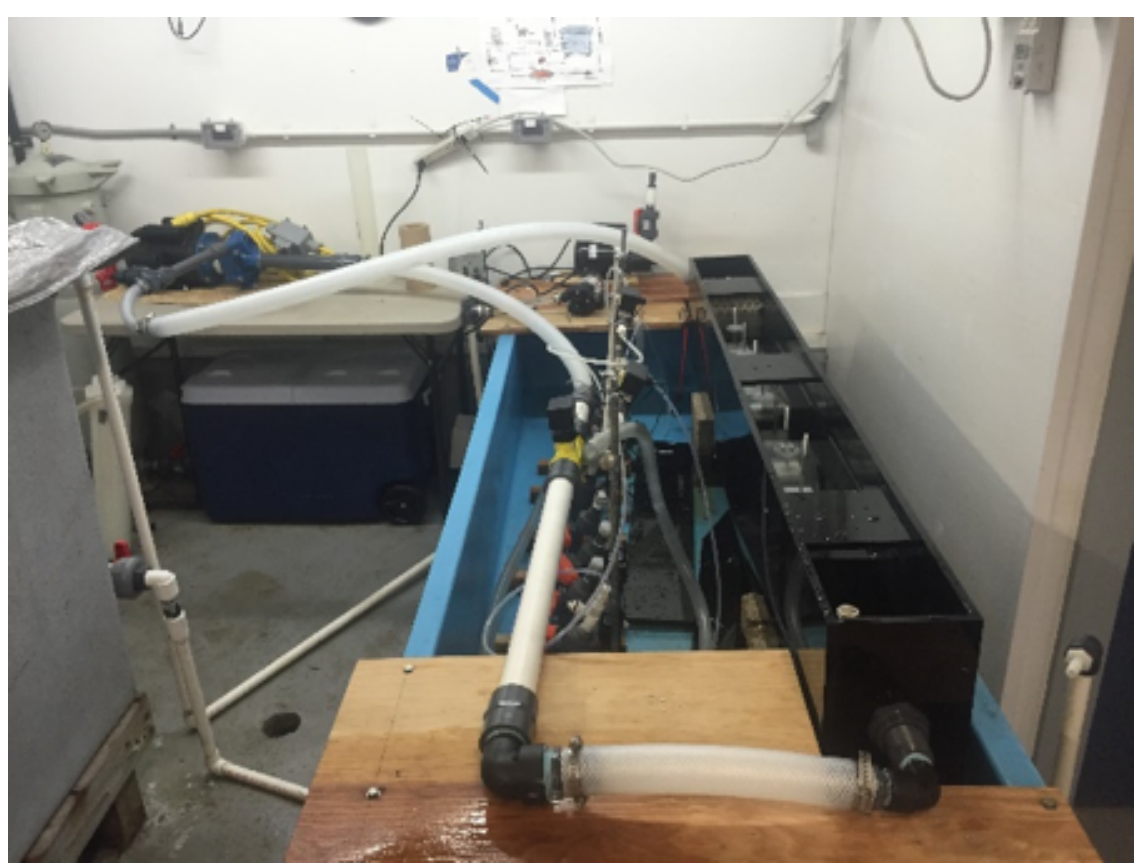

Figure 4-5: Flume tank setup with flow meter, centrifugal pump, and globe valve connected.

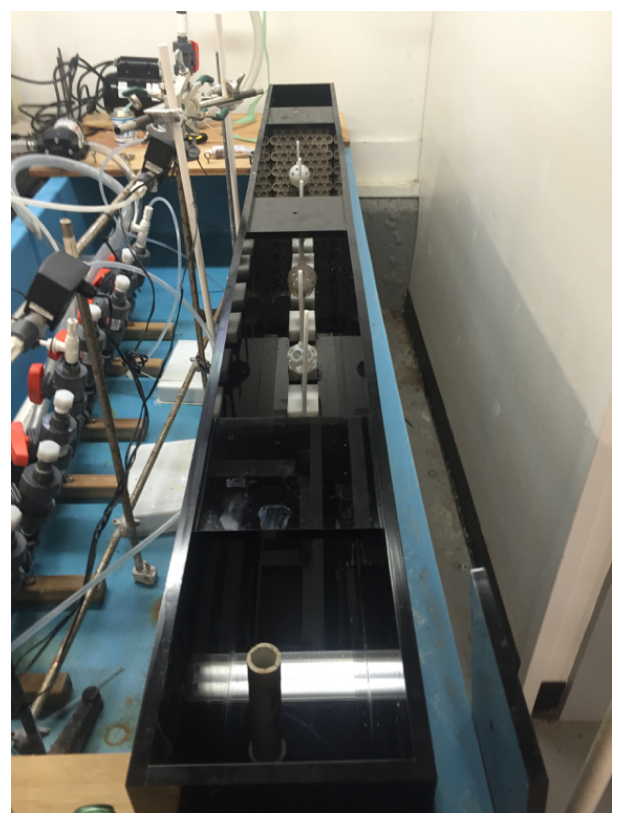

Figure 4-6: All six shells positioned in the flume tank.

of plastic, thereby minimizing the possibility of contaminating the adsorbents with other metal ions. 


\subsubsection{Design Analysis}

In order to ensure dynamic similarity between the experiment and a full-scale scenario, Reynolds number scaling was used. For a shell of diameter $d$, the Reynolds number is given by

$$
\operatorname{Re}=\frac{u d}{\nu}
$$

where $u$ is the free stream velocity and $\nu$ is the kinematic viscosity. The kinematic viscosity of seawater at atmospheric pressure, salinity of $35 \mathrm{psu}$, and temperature of $20^{\circ} \mathrm{C}$, is $\nu=1.05 \times 10^{-6} \mathrm{~m}^{2} / \mathrm{s}$. For a full-scale shell of $500 \mathrm{~mm}$ in a flow of $10 \mathrm{~cm} / \mathrm{s}$ (on the lower end of what is expected in an ocean setting), $R_{\text {full-scale }}=4.76 \times 10^{4}$. For a $50 \mathrm{~mm}$ diameter shell being tested in the flume at $4.8 \mathrm{~cm} / \mathrm{s}$, the Reynolds number is $R e_{\text {model }}=2.29 \times 10^{3} . R_{\text {full scale }}>10^{4}$ indicates the flow is in the turbulent regime, whereas $R e_{\text {model }}<10^{4}$ suggests the flow may not be turbulent in the flume.

To further ensure that the same physical phenomena were being witnessed in the flume as in the open ocean, the drag coefficient at various Reynolds numbers for the case of the standard wiffle ball (enclosure C) were further investigated. If the drag coefficient at $\mathrm{Re}_{\text {model }}$ was found to be similar to be $\mathrm{Re}_{\text {full scale }}$, it can be concluded that the shells in the model and at full scale would likely be seeing the same drag force due to the flow. This would suggest that the results of the flume experiment would be indicative of a full scale test in the ocean.

The drag coefficient and Reynolds number at linear flow rates ranging from 1.8 to $10 \mathrm{~cm} / \mathrm{s}$ were calculated using FlowSimulation, CFD modeling software, for a shell 50 $\mathrm{mm}$ in diameter (1/10th physical scale) as well as a $500 \mathrm{~mm}$ diameter (full scale) shell. Additionally, a Tow Tank experiment was used to experimentally verify the drag coefficient, $C_{D}$, for a solid sphere and wiffle ball for a series of Reynold's numbers (Hamlet, 2017). As can be seen from figure 4-7, the experimentally determined $C_{D}$ for the case of the wiffle ball enclosure (indicated by the green diamonds) ranges from 0.9-0.7, whereas the $C_{D}$ determined from simulations of the wiffle ball enclosure (indicated by the red asterisks and yellow circles) range from 0.75-0.5. Although the experimentally and flow simulated drag coefficients of the wiffle ball do not agree exactly, they do exhibit the same trend: decreasing drag with increasing Reynold's number, which is indicative of turbulent flow. Additionally, in the experimental regime of the flume test (indicated by the black circle) the drag coefficient has decreased, suggesting the onset of turbulent flow. Thus, from these results it can be concluded that the forces seen on the shells in the flume test are likely indicative of those that would be seen by a full-scale shell in the ocean.

The shell locations within the flume were chosen to ensure shells would not lie in the boundary layer created by the walls of the flume or in the wake created by shells upstream. The free stream velocity is given by the volumetric flow rate divided by the cross sectional area of the flume:

$$
u=\frac{Q}{w_{f} d_{f}}
$$




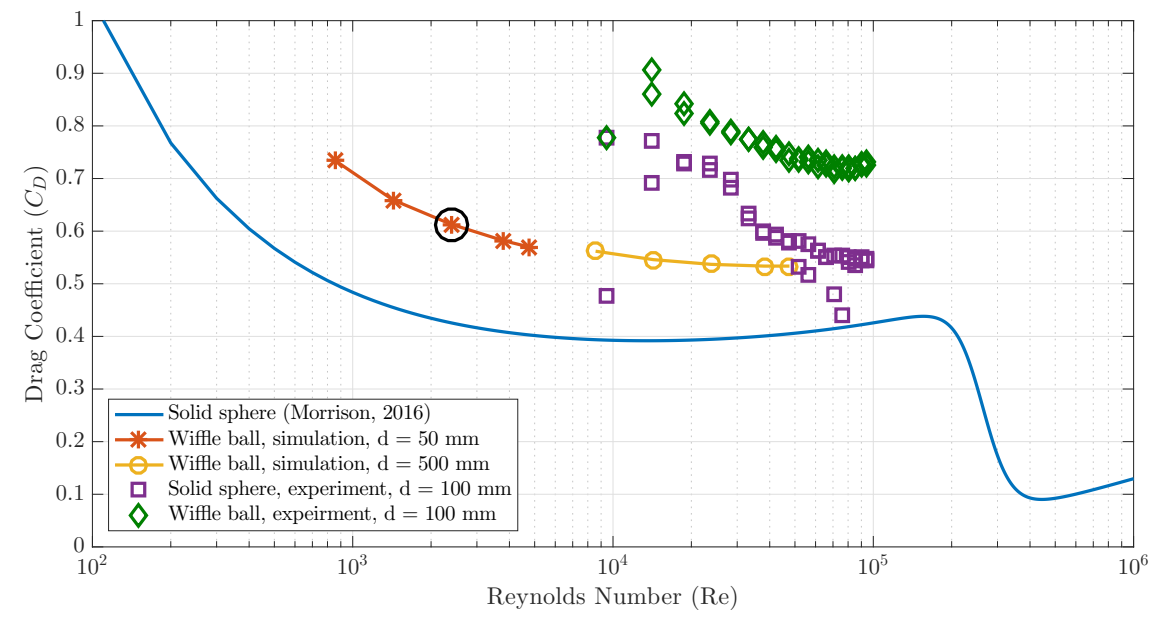

Figure 4-7: Drag coefficient as a function of the Reynolds number for a solid sphere as predicted by Morrison, 2016 (solid blue line), a wiffle shell with diameter $50 \mathrm{~mm}$ (red stars) and $500 \mathrm{~mm}$ (yellow circles) as determined from CFD simulations, as well as those determined by Tow Tank drag experiments for a solid sphere (purple squares) and a wiffle ball (green diamonds) of diameter $100 \mathrm{~mm}$. The black circle indicates the experimental regime of the flume test described in this chapter. Tow Tank drag experiments were performed by Amanda Hamlet (Hamlet, 2017)

where $u$ is the free stream velocity, $Q$ is the volumetric flow rate, $w_{f}$ is the width of the flume and $d_{f}$ is the depth of the water filling the flume. The flow regime at any point in the flume can be determined from the local Reynolds number, given by:

$$
\operatorname{Re}(x)=\frac{u x}{\nu}
$$

where $\operatorname{Re}(x)$ is the local Reynolds number, $u$ is the free stream velocity, $x$ is the distance downstream from the boundary layer, and $\nu$ is the kinematic viscosity of seawater.

Since the fluid's velocity asymptotically approaches the free stream velocity, the thickness of the boundary layer is commonly taken as the point where the fluid velocity equals $99 \%$ of the free stream velocity. From (4.3), it was found that any free stream velocity greater than $0.92 \mathrm{~cm} / \mathrm{s}$ resulted in a non-laminar flow regime within the flume, for which the boundary layer thickness is then given by:

$$
\delta=\frac{0.382 x}{\operatorname{Re}(x)^{1 / 5}}
$$

where $\delta$ is the boundary layer thickness, $x$ is the distance downstream from the start of the inlet, and $\operatorname{Re}(x)$ is the local Reynolds number.

With this information, for a flow rate of $4.8 \mathrm{~cm} / \mathrm{s}$ and a shell spacing of $5 \mathrm{in}$, the boundary layer at the last shell would be approximately 1.77 in thick (on either side of the tank walls), meaning that the flow in a shell with a diameter greater than 2.45 in would be affected by the boundary layers. A shell with a diameter of $50 \mathrm{~mm}(1.97$ in), as those used in this experiment, should not be affected by the boundary layer. 


\subsubsection{Adsorbent preparation, sampling, and analysis}

Each shell contained a pre-weight, small mass of adsorbent fiber cut from a common AI8 adsorbent braid prepared by Oak Ridge National Laboratory Gill et al. (2016). Samples were collected after the first 24 hours and then again once per week for eight weeks, for a total of nine samples over a 56-day test. Samples were taken by snipping small pieces $(\sim 35 \mathrm{mg})$ of the braid in each enclosure at these different time points. This allowed for the study of the adsorption kinetics of uranium and other trace elements. The adsorbent samples were analyzed at the Marine Sciences Lab at Pacific Northwest National Laboratory. In addition to adsorbent samples, salinity measurements were taken using a YSI Pro30 Conductivity Probe and water samples were also collected on each of the nine sample dates for trace metal analysis.

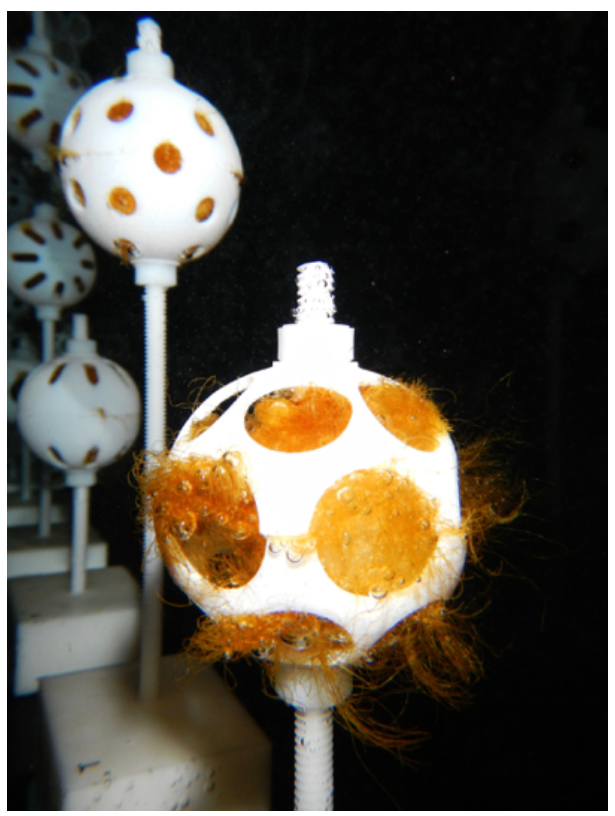

Figure 4-8: Photography showing the adsorbent in shells in the flume.

\subsection{Results}

Due to the conservative behavior of uranium in seawater, (Not et al, 2012) all uranium adsorption capacity data was normalized to a salinity of $35 \mathrm{psu}$ in order to correct for the varying salinity of natural seawater observed in different adsorption experiments. Adsorption kinetics and saturation capacity were determined by fitting time-dependent measurements of adsorption capacity using a one-site ligand saturation model as described by ([2.2). Figure 4-9] shows the time-dependent measurements of adsorption capacities for all trace metals retained by the AI8 adsorbent for each enclosure. As can be seen, uranium is not the dominant metal adsorbed by the fiber.

The uranium adsorption capacity ( $\mathrm{g}$ - $\mathrm{U} / \mathrm{kg}$-adsorbent) for all adsorbents in all the enclosures is shown in figure 4-10, with the lines indicating the one-site ligand 
saturation model fits for each enclosure. The results from a flume study conducted in 2015 of the AI8 fiber alone are also included in the graphic. As can be seen, there is good agreement between the 2015 flume study and the study presented here. Furthermore, there is very little difference in the uranium adsorbed between the different enclosure types. This suggests that the shell enclosure is likely not inhibiting the uranium adsorption of the fibers it encases, no matter the shell design.

The saturation capacity, $\beta_{\max }$, and half saturation time, $K_{D}$, as well as the uptake predicted for an immersion time of 56 days as predicted from the one-site ligand modeling (equation ( $(2 \cdot)$ ), is shown in figure $4-1]$. The error bars detail the $\pm 95 \%$ confidence intervals of the coefficients. As shown in the figure, there is little variability between the coefficients of the fits for each shell enclosure, suggesting no significant difference in the uranium uptake by the adsorbent in different shell enclosures.

This result is further seen in figure 4-12 which shows the total uranium adsorbed (g $\mathrm{U} / \mathrm{kg}$ adsorbent) at day 56 for the adsorbent fibers in each of the different enclosures. Enclosure F resulted in the highest uranium uptake of $3.74 \mathrm{~g} \mathrm{U} / \mathrm{kg}$ adsorbent, whereas enclosure A saw the least amount at $3.23 \mathrm{~g} \mathrm{U} / \mathrm{kg}$ adsorbent, and the control with no enclosure saw an uptake of $3.54 \mathrm{~g} \mathrm{U} / \mathrm{kg}$ adsorbent. The difference in uranium adsorbed by enclosure $\mathrm{F}$ and $\mathrm{A}$ was only $13.5 \%$, and the difference between the uranium adsorbed by any enclosure and the control adsorbent was at most $8 \%$. This suggests, again, that there was no significant difference in the uranium adsorbed by fibers in the shells as compared to the control, and no significant difference between the uranium adsorbed between the different enclosure designs. 

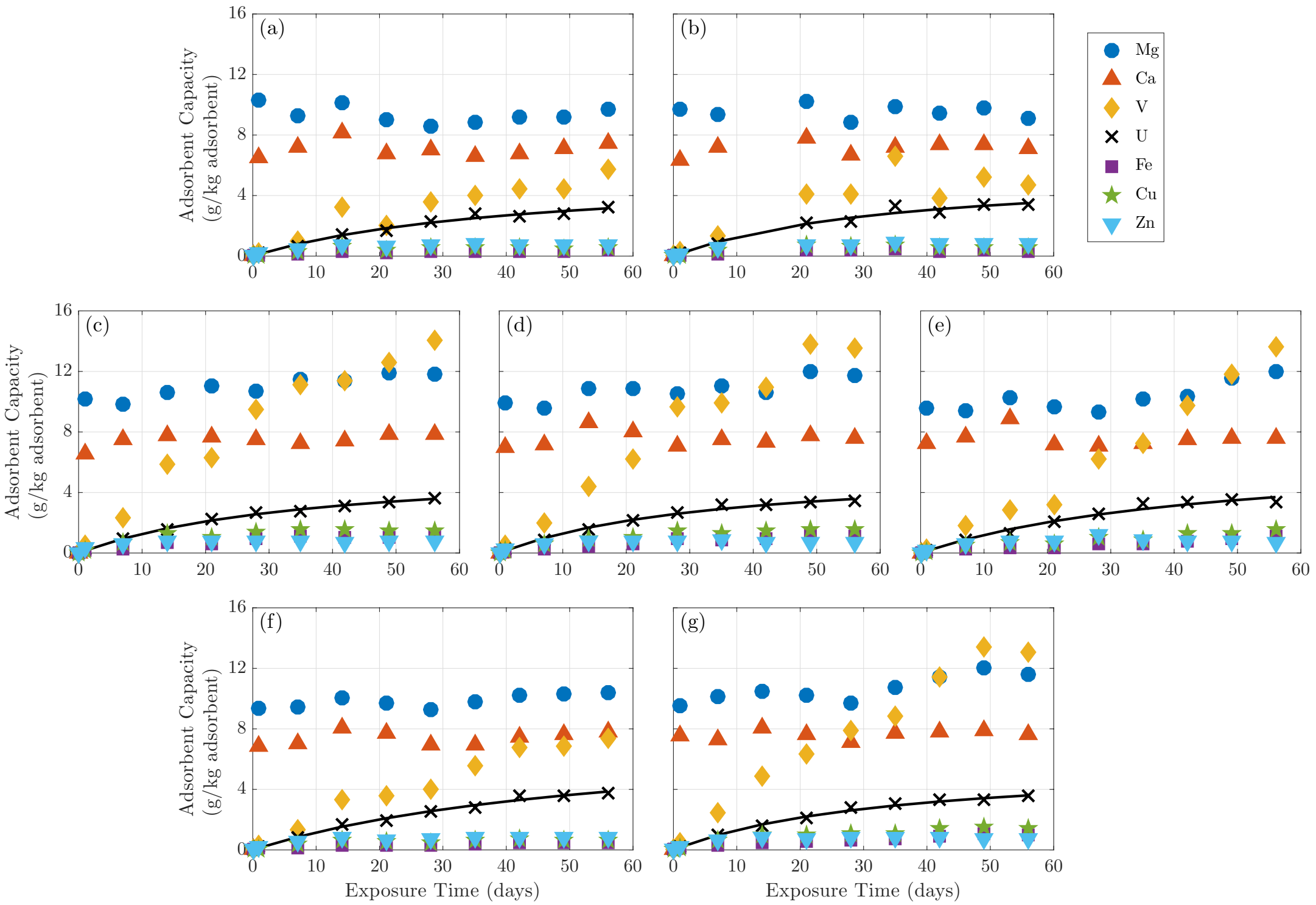

Figure 4-9: Time-dependent measurements of adsorption capacities (g element/kg adsorbent) for several trace elements retained by the ORNL AI8 adsorbent exposed to filtered natural seawater in a flume in various shell enclosures, (a)-(f) (as shown in figure (4-3), and without any enclosure, (g). The black line drawn through the uranium adsorption data represents fitting to a one-site ligand model (equation ([2.2)) 


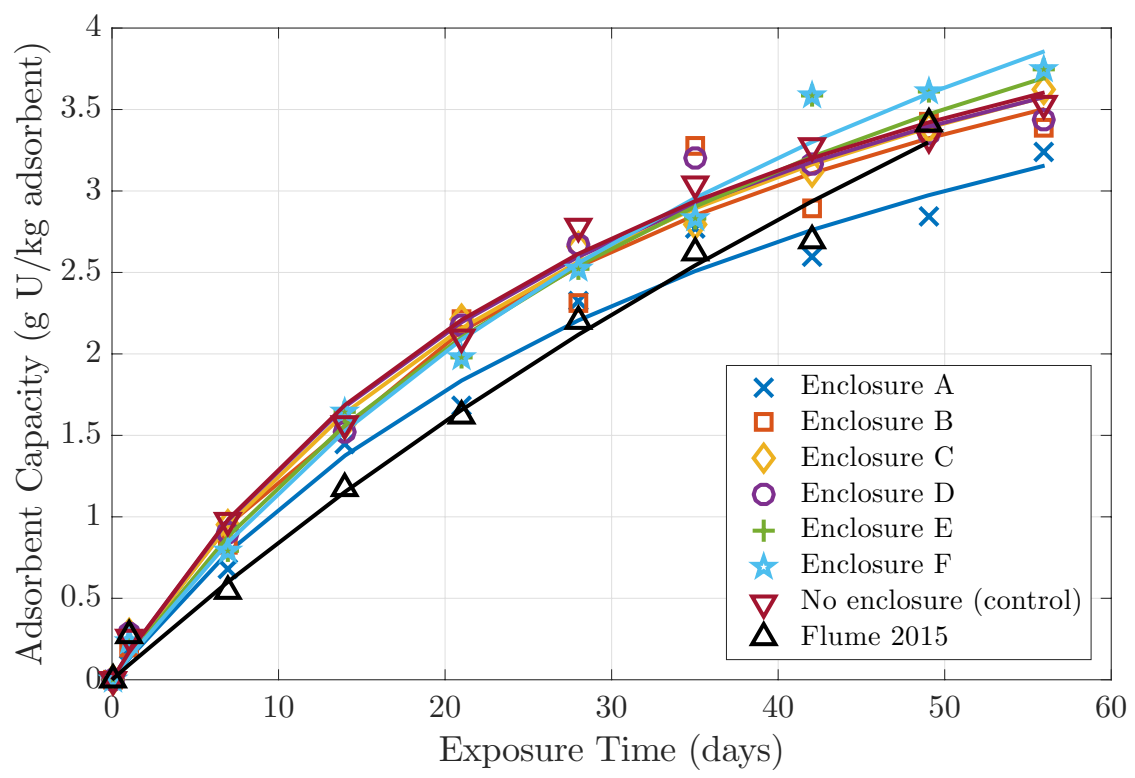

Figure 4-10: Time-dependent measurements of uranium adsorption capacity (g-U/kgadsorbent) for the flume experiment for the seven AI8 adsorbent braids enclosed by various shell designs (Enclosures A-F), as well as the results from the control adsorbent in no enclosure and the results from a flume experiment conducted in 2015 on AI8 fibers alone. The uranium adsorption capacity was normalized to a salinity of 35 psu. Curves drawn through the data represent fitting to a one-site ligand model (equation( $(2-2)$ ). Figure 4-T details the saturation capacity and half-saturation times as predicted from the one-site ligand modeling
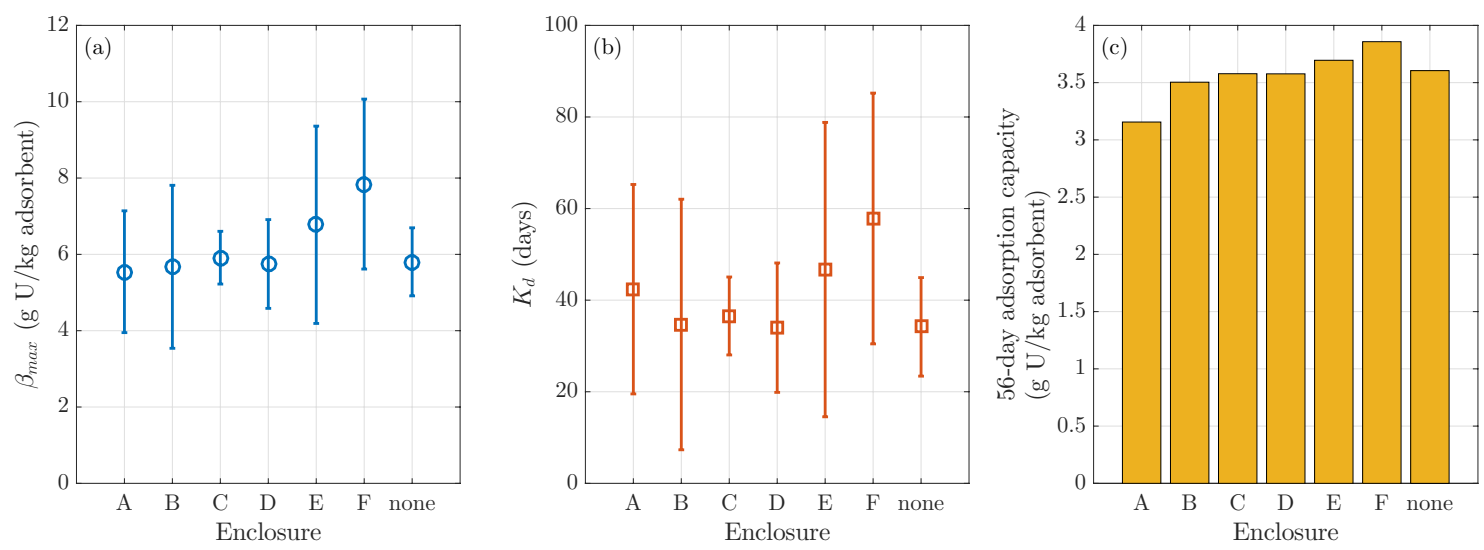

Figure 4-11: The (a) saturation capacity, $\beta_{\max }$, (b) half saturation time, $K_{D}$, and (c) 56 -day uranium uptake predicted by the one-site ligand model (equation (2.2))

\subsection{Discussion}

Results from this experiment will help inform the types of protective shell enclosures to be used in a large-scale ocean test of a uranium harvesting system. The flow rate of $4.8 \mathrm{~cm} / \mathrm{s}$ used in this experiment, while chosen so as to reduce the boundary layer 


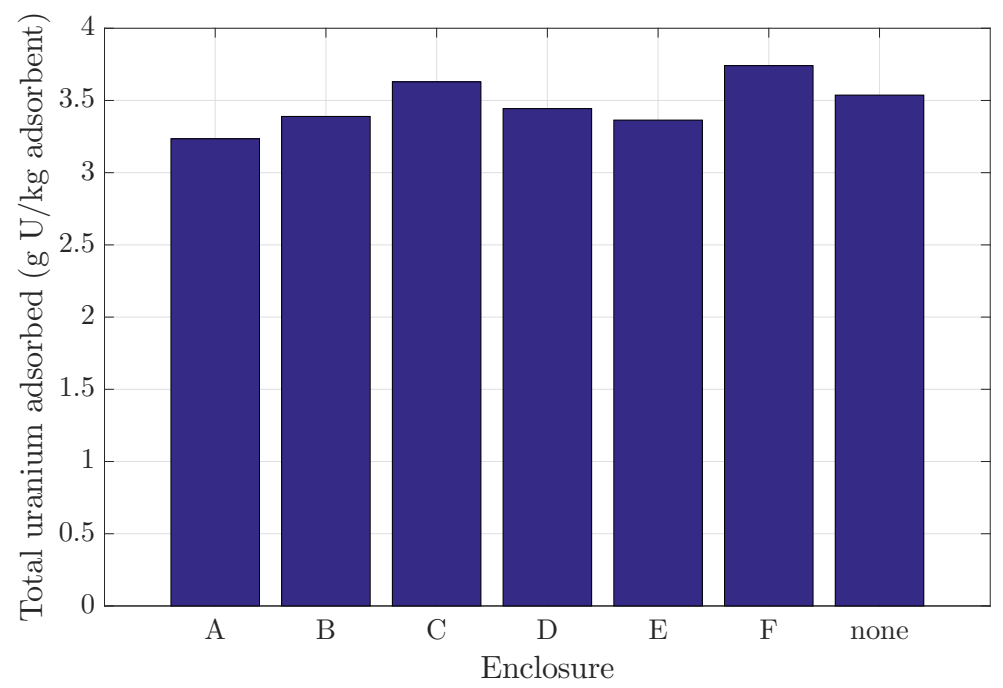

Figure 4-12: The total uranium adsorbed at day 56 by the adsorbent fibers in each enclosure from the flume experiment.

effects and ensure similarity between the forces on the shells in the flume as in the open ocean, is much lower than is expected in the ocean of eastern boundary currents, which can range from 10-30 cm/s. These are locations that have been hypothesized to be the best for the deployment of a uranium harvesting system in order to maximize the water flow seen by the adsorbent.

There was no difference in the amount of uranium adsorbed by the fibers in each of the shell enclosures, suggesting that the enclosures do not inhibit the uptake of uranium. It is highly unlikely that the flow field is the same inside each shell (a topic being investigated by Amanda Hamlet (Hamlet, 2017)). Thus, the lack of difference in uranium uptake between shells suggests that the linear velocity of the water is high enough that the reaction is no longer mass limited, as evidenced by Ladshaw et al. (2017) for similar linear velocities.

Additionally, experiments should investigate how the fiber may survive in such strong currents. Biofouling of adsorbent fibers can have an adverse effect on uranium uptake (Park et al, 2016), and future work should examine the effects of adsorbent shell enclosures on the biofouling of adsorbent fibers. Lastly, further structural analysis of protective shell enclosures used in this experiment should be conducted to determine the effects of distributed and point loads on different shell geometries. 


\section{Chapter 5}

\section{Symbiotic Machine for Ocean uRanium Extraction (SMORE)}

This chapter describes the design of the Symbiotic Machine for Ocean uRanium Extraction (SMORE).

\subsection{Functional Requirements}

The functional requirements of a symbiotic device to harvest uranium from seawater are:

1. use the amidoxime-based polymeric adsorbent developed by Oak Ridge National Laboratory known as AI8 Gill et al (2016);

2. recover 1.2 tonnes of uranium from seawater per year, enough annual fuel to power a 5-MW nuclear power plant annually; and

3. bring the cost of uranium extraction from seawater as close as possible to terrestrial uranium mining.

The uranium uptake for this system was predicted using the one-site ligandsaturation model, equation ([2.2). Further analysis on the adsorbent behavior, recovery rate, and degradation (as described in chapter $\nabla$ ), reveals that in order to achieve functional requirement (2), the sorption process can be optimized on the device using approximately 45 tonnes of adsorbent that is submerged in seawater for 23 days and cycled 15 times. While (22) is an idealized model not taking into account temperature, which has been found to have a large impact on the uptake of uranium as described in chapter 2, it allows for a starting point in determining the mechanical design of a potential system. In general, the immersion time and number of elution cycles, and hence total amount of adsorbent required, can be left as variables that propagate through the design tool, thereby allowing for the quick analysis of numerous designs. 


\section{$5.2 \quad$ Elution and Regeneration}

Unlike previous designs developed by Picard et al. (2014) and Haji et al. (2016), which utilize on-site continuous acidic elution and bicarbonate regeneration processes, this design employs a single, 24-hour bicarbonate elution as described by Pan et al. (2017). Recent work has shown that the acidic elution process leads to degradation of the adsorbent with subsequent reuse, which may be mitigated or removed altogether by the replacement of acid by a potassium bicarbonate solution (Pan et al, 2017). Additionally, the adsorbent no longer needs to be regenerated with alkaline solution since a basic solution has replaced the previously used acids. The elimination of this step provides a significant cost savings through the reduction of chemical consumptions (Byers, 2015).

\subsection{Mooring and Recovery}

Uranium-adsorbing materials with the optimal chemical properties for high adsorbent capacity, in general, have inherently low tensile strength and durability, as described in chapter 3 . Hence, the designs previously studied by Picard et al. (2014), which require the adsorbent to be woven into a belt which is held in tension, are likely not feasible with the AF1 adsorbent, as it will probably not possess the necessary durability and tensile strength required. The design presented here utilizes the shell enclosure strategy described in chapter 3 in which the mechanical and chemical requirements of the system are decoupled. In these designs and the system presented in this chapter, the uranium adsorbent material with high adsorbent capacity is enclosed in a hard permeable outer shell with sufficient mechanical strength and durability for use in an offshore environment and chemical resilience against elution treatments.

\subsection{Mechanical Design}

Because offshore wind turbine data is proprietary and often very difficult to use for public work, the present design for SMORE utilizes the StatOil OC3-Hywind spar with a 5-MW National Renewable Energy Laboratory (NREWL) turbine, a device well documented by NREL (Jonkman et al., 2009; donkman, 2010). This wind turbine, as shown in figure 5-1, has a total draft of $D_{\text {system }}=120 \mathrm{~m}$, an upper spar diameter of $d_{\text {upper }}=6.4 \mathrm{~m}$ and a lower spar diameter of $d_{\text {lower }}=9.4 \mathrm{~m}$ (Ionkman, (2010).

Design analysis and prototype testing by Haji et al. (2016) found that devices which used multiple subsystems for a uranium harvester allowed for a higher device uptime because complications that arise at sea were highly unlikely to affect all subsystems. However, because the cost of such a device is closely related to the material required, the considerable number of large gears to move the ball-chain enclosures suggest that the designs investigated by Haji et al. (2016) are likely to be extremely costly to fabricate, deploy, and maintain (Haji et al., 2017). The design presented 


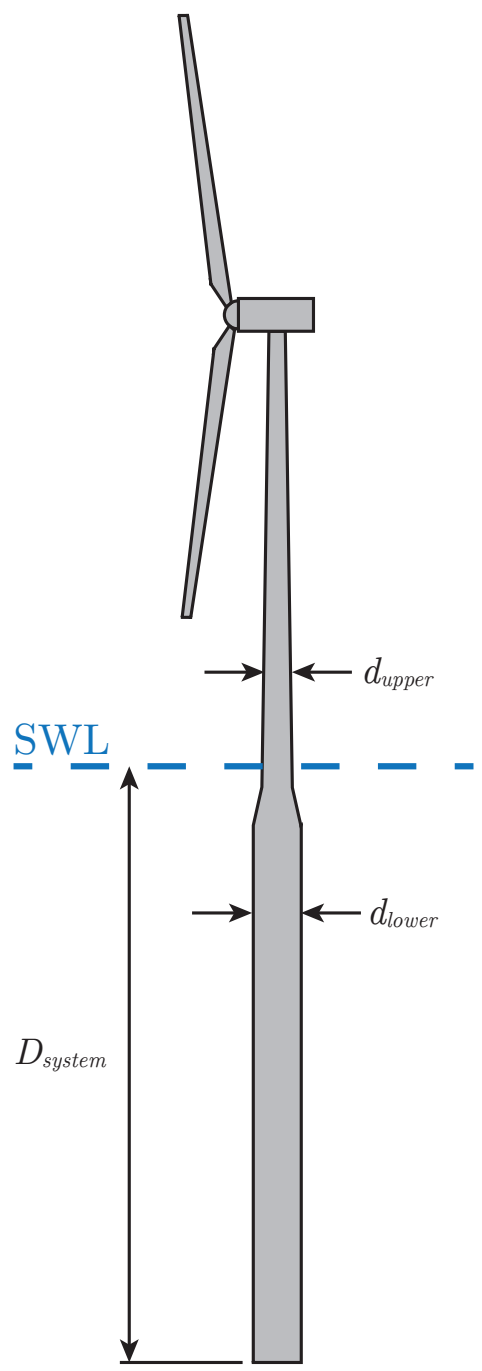

Figure 5-1: Schematic of 5-MW NREL-OC3-Hywind offshore wind turbine used as the base of the design of SMORE where SWL is the still water line. The turbine has a total draft of $D_{\text {system }}=120 \mathrm{~m}$, an upper spar diameter of $d_{\text {upper }}=6.4 \mathrm{~m}$ and a lower spar diameter of $d_{\text {lower }}=9.4 \mathrm{~m}$ (Jonkman, 2010).

here utilizes adsorbent shells that are incrementally paced along high strength mooring rope, resembling conventional ball-chain belts, similar to those in the designs by Haji et al. (2016). The design described in this chapter mimics the modularized design structure of Haji et al. (2016), while using drastically fewer components. This design, referred to as the Symbiotic Machine for Ocean uRanium Extraction (SMORE), uses large rollers to move multiple ball-chain lengths at once. A simple schematic of a version of SMORE is shown in figure 5-2.

\subsubsection{Ball-Chain Net}

In this design, as in Haji et al. (2016), the shell enclosures of diameter $d_{s}$ containing the adsorbent fibers are strung along high-strength marine grade rope, into a component 
(a)

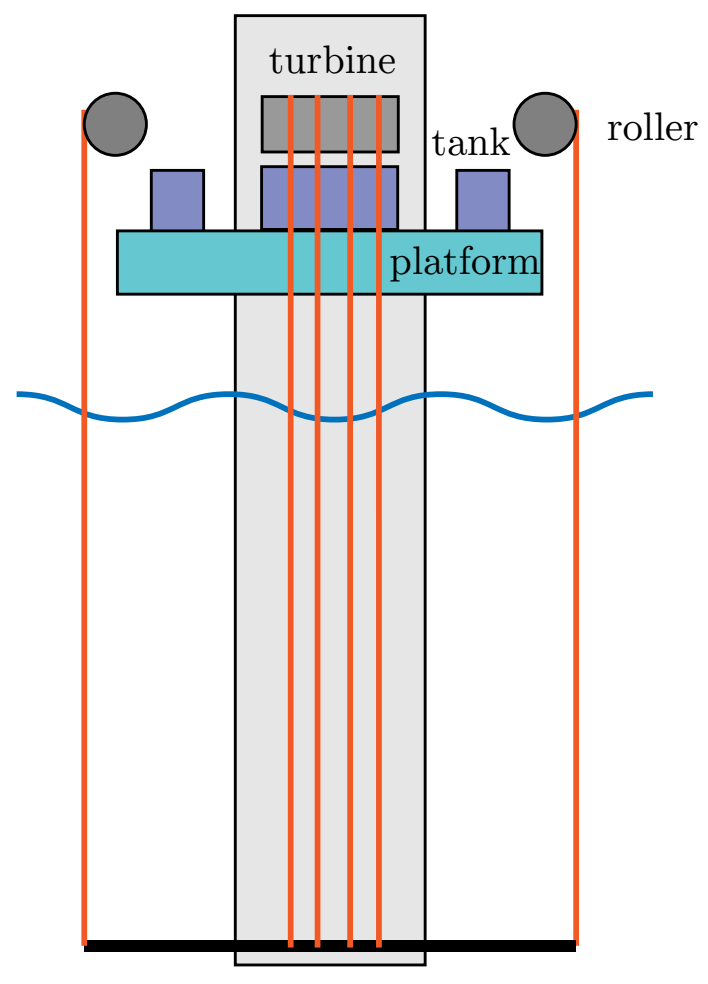

(b)

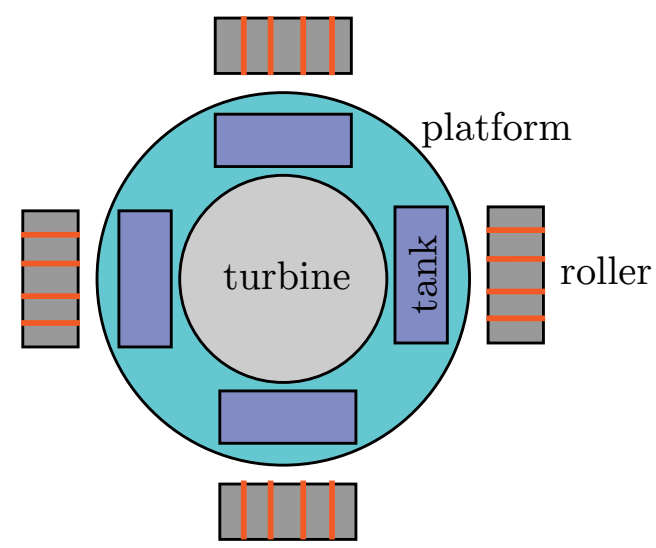

Figure 5-2: Schematic of the Symbiotic Machine for Ocean uRanium Extraction (SMORE). In this design, large rollers are used to move multiple ball-chain lengths of shell enclosures containing adsorbent fibers.

known as a ball-chain length for this design. The spacing between shells is given by $L_{s}$. Unlike the design in Haji et al. (2016), these lengths are then also placed together in multiple rows of spacing $L_{b}$ such that cross-members of high-strength marine grade rope can be added after a certain number of shells in order to develop a net-like structure. These cross-members decrease the likelihood of tangling between ball-chain lengths and increase the rigidity of the overall component. A schematic of a ball-chain net is shown in figure [5-3, where the number of ball-chain lengths per net is $N_{b}=4$.

Assuming a constant degradation of $d=5 \%$ with each elution cycle, an operating temperature of $T=20^{\circ} \mathrm{C}$, the sorption optimization algorithm developed in chapter Q2, indicates the device must be sized for approximately 45 tonnes of adsorbent. Note that the effects of biofouling are neglected for this first order analysis. Optimization on the design feasibility of winding the adsorbent fiber into filament balls as described in chapter 3 shows this can feasibly be done with a shell diameter of $d_{s}=0.5 \mathrm{~m}$ and a total number of shells of $N_{s t}=11,064$. For this case study, the spacing between shells, $L_{s}$ is taken to be $0.05 \mathrm{~m}$. 


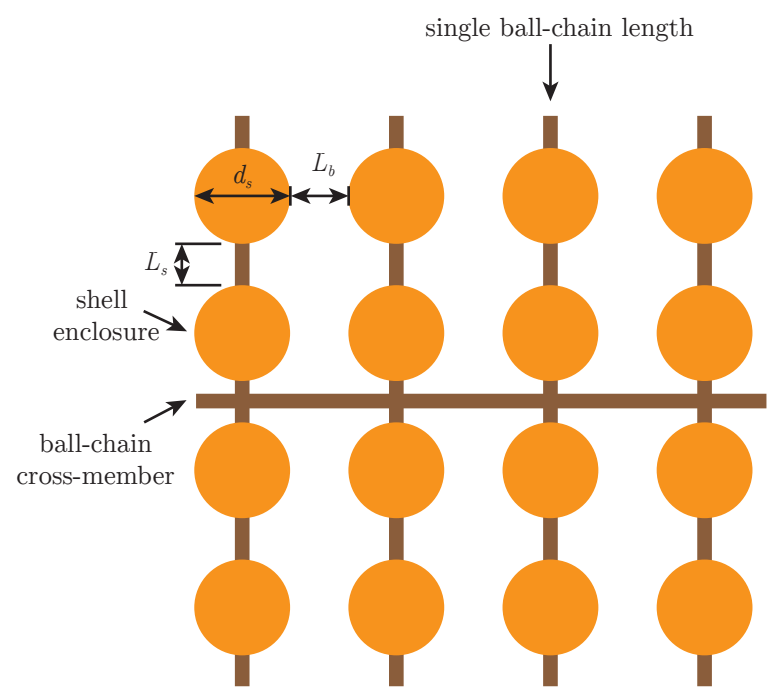

Figure 5-3: Schematic of a ball-chain net. In this image, the number of ball-chain lengths $N_{b}=4$.

\subsubsection{Roller design}

In SMORE, the adsorbent ball-chain nets are engaged and motorized by a roller. Each roller is sized to fit 12 shell enclosures around its circumference and the number of rollers per device is determined by the overall adsorbent required.

The roller diameter is determined by

$$
d_{\text {roller }}=\frac{\sqrt{d_{s}^{2}+L_{s}^{2}-2 d_{s} L_{s} \cos (\alpha)}}{2 \sin \left(\frac{\pi}{2 N_{s, r}}\right)},
$$

where $d_{s}$ is the shell diameter, $L_{s}$ is the spacing between shells on the same ball-chain length, $\alpha$ is the angle separating the midpoints of the irregular polygon created by the shells and spaces that make up the circumference of the roller (see figure 5-4, and $N_{s, r}$ is the number of shells engaged by half of the roller, taken to be six in this design (so that the roller has places for 12 shells total around its circumference). By geometry, the angle $\alpha$ is given by

$$
\alpha=\pi\left(1-\frac{1}{2 N_{\text {roller }}}\right) .
$$

Equations (5.7) and (5.2) result in a roller outer diameter of approximately $d_{\text {roller }}=$ $2.3 \mathrm{~m}$, and length of

$$
L_{\text {roller }}=N_{b}\left(L_{b}+d_{s}\right)+2 N_{b}+2 d_{\text {rail }}
$$

where $d_{\text {rail }}$ is the diameter of the circular rail on the inside edges of the roller that act like a track for a set of U-groove wheels that rotate the roller. These dimensions are discussed in section 5.4.5. For this case study, the desired number of subsystems is four, hence $N_{b}=10$ ball-chain lengths per roller. These rollers can be manufactured 


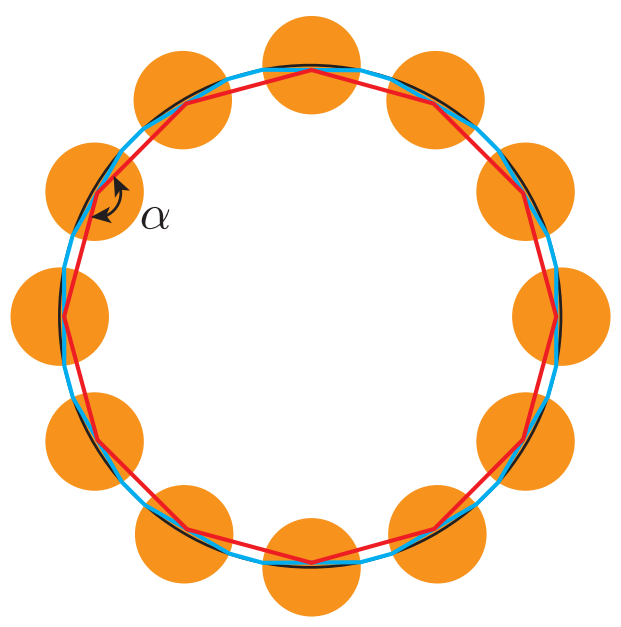

Figure 5-4: Schematic describing $\alpha$. in terms of the shells (orange) around the roller (black line). The blue line is the irregular polygon created by the shells and the spaces that make up the circumference of the roller and the red line is the regular polygon inscribed by the midpoints of the irregular polygon sides. Here $N_{s, r}=6$.

out of steel pipe, sized to be at least $0.05 \mathrm{~m}$ thick.

\subsubsection{Platform length}

To determine the platform length, a spacing of $L_{s p, r o l l e r}=1 \mathrm{~m}$ between rollers is assumed. With this in mind, the total platform length required to fit all the rollers around the turbine (such that when viewed from above the turbine is circumscribed by a polygon made up of the rollers and spaces between them, a similar analysis as was done to determine the size of the roller to hold the desired number of shells around its circumference) is given by

$$
L_{p t}=\frac{N_{\text {roller }}\left(L_{\text {roller }}+L_{s p, \text { roller }}\right)}{2 \pi}-r_{\text {upper }}-\frac{d_{\text {roller }}}{2},
$$

where $N_{\text {roller }}$ is the number of rollers (four for this design), $L_{\text {roller }}$ is the length of the roller, $L_{s p, \text { roller }}$ is the spacing between rollers, and $r_{\text {upper }}$ is the radius of the turbine at the top $\left(r_{\text {upper }}=\frac{d_{\text {upper }}}{2}\right)$. Equation (5.4) yields a platform that must be at least $L_{\text {platform }}=2.74 \mathrm{~m}$ long, from the edge of the turbine. Furthermore, to ensure that the rollers are out of water and not impacted by slamming loads due to waves, the platform would be raised approximately $H_{\text {platform }}=10 \mathrm{~m}$ above the sea surface. Further analysis for a real ocean implementation would require that this height be adjusted according to the wave climate of the region of deployment.

\subsubsection{Elution and Regeneration}

Given that the bicarbonate elution process requires 24 hours of polymer immersion time and an additional 3 hours for regeneration, the rollers on SMORE would act mostly as anchors for the ball-chain lengths hanging off the system. The rollers would 
be motorized so that after a campaign length (taken to be 23 days for this design), the ball-chain lengths could be pulled up and deposited into a chemical tank beneath the rollers. This tank would then be filled with the solution required for elution of the adsorbent polymer. After 24 hours, the rollers would be powered in the opposite direction to redeploy the uranium adsorbent. One chemical tank is envisioned per subsystem. Figure 5-5 describes the elution and regeneration process including the chemicals and tanks involved. Further design analysis is required to determine the details of each of these tank systems which was not the primary focus of this thesis.

\subsubsection{Roller and Wheel Subassembly}

As described previously, each roller would be motorized in order to wind and unwind the ball-chain lengths. This is accomplished using grooved wheels on a circular track welded to the interior of the roller on both ends. The number of grooved wheels required is determined by using the maximum contact pressure for an ellipsoid region of Hertz contact between the wheels and the groove, given by

$$
P_{\max }=\frac{3 F_{c}}{2 \pi c d}
$$

where $F_{c}$ is the contact force between each wheel and the rail (taken to be half the force on the roller divided by the number of wheels) and $c$ and $d$ are the major and minor contact area elliptical semi-axes, respectively. The semi-axes are a function of the geometry and material of the wheel and rail, and the angle between the planes of principal curvature of the two bodies. Their definitions can be found in Slocum (20)8).

In order for the design to be feasible, two criteria must be met with respect to the wheels:

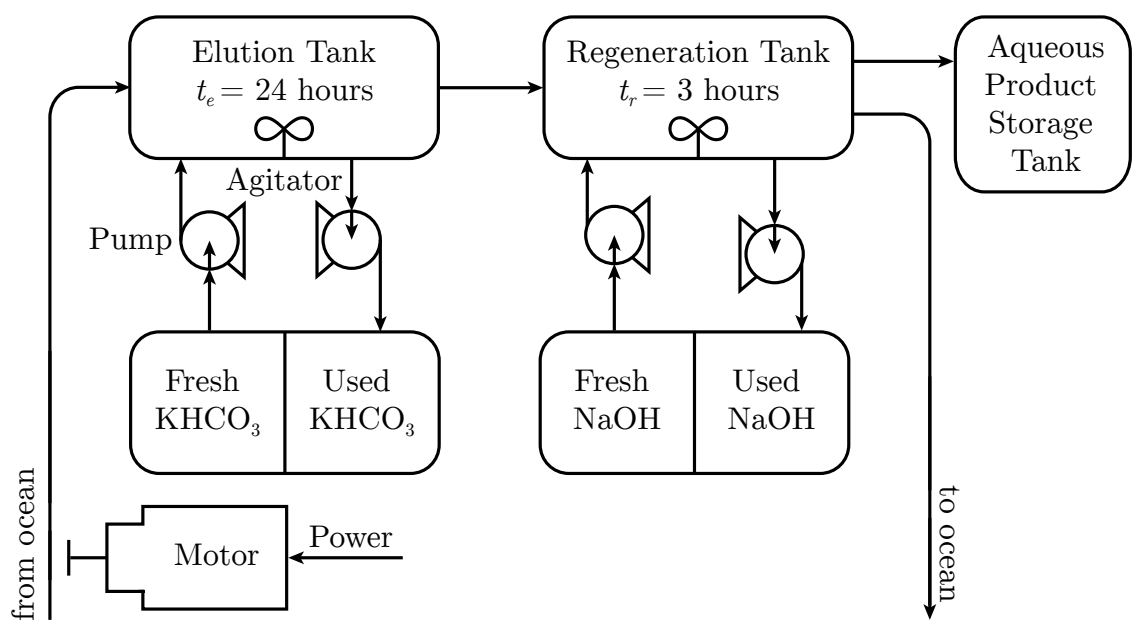

Figure 5-5: Schematic describing elution and regeneration process of polymer adsorbent. 


$$
P_{\max } \leq 1.5 \sigma_{\text {ult }}
$$

and

$$
\gamma>20^{\circ}
$$

where $\sigma_{u l t}$ is the ultimate tensile strength of the grooved wheel and $\gamma$ is the contact angle in degrees between the groove and the rail, found by

$$
\gamma=\tan \left(\frac{r_{\text {groove }}}{b}\right)^{-1},
$$

where $r_{\text {groove }}$ is the radius of the groove on the wheel. Analysis using equations (5.5)-(5.8) suggests that seven polyurethane wheels of approximately $d_{\text {wheel }}=0.4$ $\mathrm{m}$ diameter with a groove diameter of approximately $d_{\text {groove }}=0.35 \mathrm{~m}$ on a $d_{\text {rail }}=$ $0.25 \mathrm{~m}$ diameter steel track bent into a $2.1 \mathrm{~m}$ diameter circle to fit inside the roller. Furthermore, a $0.25 \mathrm{~m}$ diameter pipe may be bent into a minimum of a four times its diameter, or a $1 \mathrm{~m}$ diameter circle ( $\mathrm{H}$ and $\mathrm{H}$ Tooling), therefore bending it to fit inside the $2.1 \mathrm{~m}$ inner diameter of the roller is feasible. To support the weight of the roller and the ball-chain lengths, each subsystem would be supported by circular steel tubing of appropriate diameter and thickness with a $45^{\circ}$ angle cross-brace. Having determined this rail diameter, the resulting roller length is $L_{\text {roller }}=6.6 \mathrm{~m}$ by equation 5.3.

In order to move the rollers, one of the polyurethane wheels would be oriented completely vertically, so as to take the total load of half of the roller, and actuated using a motor. The torque required to move the roller due to friction between the wheel and the rail is

$$
\tau_{\text {fr }}=\mu F_{N}\left(\frac{d_{\text {roller }}}{2}-d_{\text {rail }}\right),
$$

where $\mu$ is the coefficient of friction between the polyurethane groove and the steel rail (taken to be $\mu=0.2$ ) and $F_{N}$ is half the total force on the roller due to the shells, its mass, and the tension on the ball-chain net. Analysis of this friction force indicates that the friction between the wheel and rail provides enough torque needed to move the rollers.

\subsubsection{Power Requirement}

The power required for the full-scale system is a combination of the power required to move the adsorbent ball-chain net using the rollers, the power required for the chemical elution and regeneration, and the power required to pump the chemicals throughout the system.

\section{Friction between wheels and rail}

In the case of the power required to move the adsorbent ball-chain, the dominant forces to overcome are the friction between the driving wheel and rail and the tension 


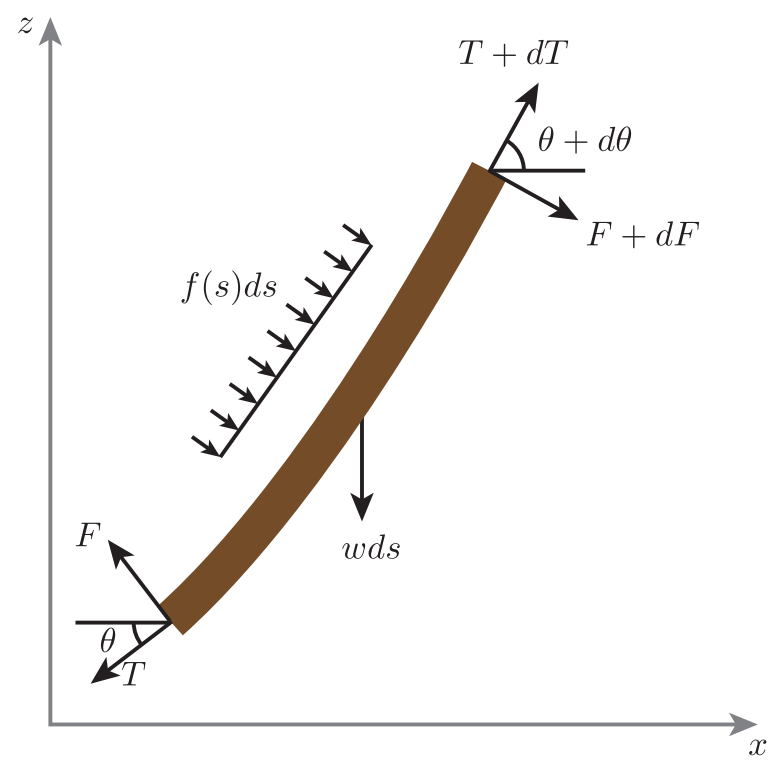

Figure 5-6: Model of small segment, $d s$, of the ball-chain under a lateral current load, $f(s)$, with constant apparent weight per unit length, $w$. The axis following the segment is given by $s, F$ is a sheer force, $T$ is the tension in the ball-chain, and $\theta$ is the angle the cable makes with the horizontal.

force required to keep the ball-chain net taut. Given $\tau_{f r}$ by equation (5.9), the power required to move the roller against this friction is

$$
P=\tau_{f r} \omega,
$$

where $\omega$ is the roller speed in $\mathrm{rad} / \mathrm{s}$.

\section{Ball-chain tension}

Following the analysis presented by Hamlet (2017), the tension force required to keep the ball-chain from deflecting under the current loads of the ocean can be found by considering the cable to be a tensioned-beam. Following the analysis described in Sparks (2007), the ball-chain rope can be considered a cable under a lateral current load. Figure 5-6 illustrates this scenario, where $f(s)$ is the force per unit length due to the ocean currents, and $w$ is the apparent weight per unit length (assumed to be constant). The angle of deflection $\theta$ is assumed to be large in this analysis.

Considering the forces normal to the segment axis and assuming static equilibrium yields

$$
F-w d s \cos (\theta+d \theta)-(F+\delta F) \cos d \theta+(T+d T) \sin d \theta-f(s) d s=0,
$$

where $F$ is the shear force and $T$ is the tension in the ball-chain. Dividing by the length $d s$, and simplifying gives, 


$$
\frac{d F}{d s}+w \cos \theta-T \frac{d \theta}{d s}+f(s)=0
$$

The shear force $F$ is equal to $\frac{d M}{d s}$, and the curvature $\frac{1}{R}=\frac{M}{E I}=\frac{d \theta}{d s}$, where $M$ represents the internal moment, $E$ is the Youngs Modulus, and $I$ is moment of inertia of the cross section (Sparks, 20107). Equation (5.12) is therefore recast as

$$
\frac{d^{2}}{d s^{2}}\left(E I \frac{d \theta}{d s}\right)-T \frac{d \theta}{d s}+w \cos \theta+f(s)=0,
$$

where $E I$ is the bending stiffness, $T$ is the effective tension, $w$ is the apparent weight per unit length, and $f(s)$ is the applied load function. A cable can be considered to be a tensioned-beam for which the bending stiffness is neglected, (i.e. $E I \approx 0$ ), which results in equation (5.T3) simplifying to

$$
\frac{d \theta}{d s}=\frac{w \cos \theta+f(s)}{T} .
$$

Similarly, considering the forces tangent to the segment, assuming static equilibrium and neglecting bending stiffness $(E I \approx 0)$, and neglecting components of $f(s)$ tangent to the ball-chain, results in

$$
\frac{d T}{d s}=w \sin \theta
$$

The current load is expressed as

$$
f(s)=\frac{1}{2} \rho C_{d} d(s) U(s)^{2},
$$

where $\rho$ is the density of seawater, $C_{d}$ is the drag coefficient, $d(s)$ is the weighted hydraulic diameter of the segment (taking into account both the shell and rope sections), and only the component of the current normal to the ball-chain is considered, that is $U(s)=u(s) \sin \theta$.

The elongation of the rope chosen to string the shells together in the ball-chain arrangement is also considered. Given the tension in a segment of the rope and the equation found for strain, the elongated length of each segment of rope is written:

$$
d s_{\text {elong }}=d s_{\text {initial }}(1+\epsilon(s)),
$$

where $\epsilon(s)$ is the strain of the ball-chain segment, which at each point can be computed from the tension.

Equations (5.14), (5.15), and (5.17) govern the forces on the ball-chain length and can be used to determine the tension, $T$, and angle, $\theta$, for each point along the ball-chain length. In order to satisfy boundary conditions which were given by the SMORE geometry, the initial tension and angle at the bottom of the roller were imposed and the Forward Euler method of numerical integration was used to calculate the position of the top end of the rope. The calculated position was compared to the known boundary condition on the top end, and if they were sufficiently similar, less 
than $10^{-4}$ difference, the initial guesses were correct. If not, the system was solved iteratively using Excel's GRG nonlinear engine to find the correct initial tension and angle.

\section{Elution and Regeneration Processes}

The chemical elution and regeneration processes require power for a propeller to agitate the chemical bath as well as temperature control for the baths. For the initial design, the agitator power was determined as a percentage of the power required in the reference case of $3 \mathrm{~kW}$, detailed by Sachde (201]) and Schneider and Sachde (2013), based on the the amount of adsorbent eluted annually by SMORE as compared to that in the reference case.

In addition to this, the chemical baths must be raised to $40^{\circ} \mathrm{C}$ for the full duration of the elution and regeneration (University of Idaho). The power required to maintain this temperature is given by

$$
P=\frac{Q}{t}=\frac{m c_{p} \Delta T}{t}
$$

where $Q$ is the energy required, $t$ is the time over which the chemical processes take place $\left(t_{e}=24\right.$ hours and $t_{r}=3$ hours for the elution and regeneration processes, respectively), $m$ is the mass of the chemicals and adsorbent required to be headed, $c_{p}$ is the specific heat of the solution (taken to be that of water for initial analysis), and $\Delta T$ is the temperature difference. For this case study, a temperature difference of $20^{\circ} \mathrm{C}$ was considered.

\section{Chemical pumping power}

For this design, it is assumed that the chemicals could be stored within the turbine itself. This is very advantageous as offshore wind turbines often require a lot of ballast mass to ensure stability. The overall weight of the reference turbine, is 7466 tonnes (lonkman, 2010). Because the numbers for the amount of ballast used for a wind turbine are proprietary and therefore very difficult to find, it is assumed that the ballast is likely no more than $10 \%$ of the overall system mass, or 747 tonnes.

The power required to pump the chemicals from the bottom of the turbine to the location of the chemical tanks on the platform can be determined from

$$
\begin{aligned}
P & =N_{p} \frac{P_{h}}{\eta_{p}} \\
& =N_{p} \frac{\rho g h_{s y s} q}{\left(3.6 \times 10^{6}\right) \eta_{p}}
\end{aligned}
$$

where $N_{p}$ is the number of pumps (determined by the number of subsystems), $P_{h}$ is the hydraulic power in $\mathrm{kW}, \eta_{p}$ is the pump's efficiency (taken to be 30\%), $\rho$ is the density of the solution being pumped (assumed to be similar to water), $g$ is gravity, 
$h_{\text {sys }}$ is the head distance over which the fluid needs to be pumped and $q$ is the flow capacity in $\mathrm{m}^{3} / \mathrm{h}$, taken to be $1 \mathrm{~m}^{3} / \mathrm{h}$ for this analysis.

\subsubsection{Design Analysis}

The detailed design analysis was incorporated into an Excel spreadsheet that allowed for the quick analysis of various designs by changing only a few constraints. For instance, the current speed in the proposed deployment location could be changed, thereby resulting in a new tension force required to keep the ball-chain net taut, and hence a new torque requirement for the roller system. Additionally, the system could be changed from a stationary system (in which the rollers are only used to wind up the adsorbent net for chemical processing) to a continuous system (in which the adsorbent net is strung between an upper and lower set of rollers such that it is moved continuously). An example design is detailed in table 5.1 and a threedimensional sketch of a 1/10th physical scale system of such a design is shown in figure 5-7.

\subsection{Chapter Summary}

This chapter described the design of a Symbiotic Machine for Ocean uRanium Extraction (SMORE), a device designed to be incorporated into an offshore floating wind turbine such as the 5-MW NREL OC3-Hywind. The driving decisions behind this design were to use as few materials as possible, to thereby reduce cost, and to utilize a shell enclosure to protect the adsorbent. This work showed that such a machine can be feasibly designed to harvest 1.2 tonnes of uranium annually, enough to provide $5 \mathrm{MW}$ of nuclear fuel. If paired with a 5-MW offshore wind turbine, SMORE can effectively double the energy harvested per square meter of ocean. A tool was developed for quickly analyzing various types of designs for SMORE such as those that use a stationary adsorbent net or a continuously moving one. This design tool will be incorporated into the determination of the uranium production cost from seawater using this deployment strategy, described in chapter $\mathbf{8}$. 
Table 5.1: Example SMORE design

\begin{tabular}{|c|c|c|}
\hline Parameter & Value & Notes \\
\hline System type & Stationary & \\
\hline Current speed, $V_{c s}$ & $1 \mathrm{~m} / \mathrm{s}$ & Reasonable for boundary currents \\
\hline Bottom current speed, $V_{c s, b}$ & $0.01 \mathrm{~m} / \mathrm{s}$ & \\
\hline Depth to bottom current, $D_{c b}$ & $25 \mathrm{~m}$ & \\
\hline Shell diameter, $d_{s}$ & $0.5 \mathrm{~m}$ & Input \\
\hline Spacing between shells, $L_{s}$ & $0.1 \mathrm{~m}$ & Input \\
\hline Shells per turbine, $N_{s t}$ & 5532 & Optimized by shell model (chapter 3 ) \\
\hline Ball-chains lengths per roller & 10 & Input \\
\hline System depth, $D_{\text {sys }}$ & $80 \mathrm{~m}$ & Input \\
\hline Platform height above SWL, $H_{p}$ & $10 \mathrm{~m}$ & Input \\
\hline Rollers, $N_{\text {roller }}$ & & \\
\hline Roller diameter, $d_{\text {roller }}$ & $2.3 \mathrm{~m}$ & \\
\hline Roller inner diameter, $d_{\text {roller }, \text { in }}$ & $2.1 \mathrm{~m}$ & \\
\hline Roller length, $L_{\text {roller }}$ & $6.6 \mathrm{~m}$ & \\
\hline Spacing between rollers, $L_{s p, \text { roller }}$ & $1 \mathrm{~m}$ & \\
\hline Platform length, $L_{p t}$ & $2.74 \mathrm{~m}$ & \\
\hline Rail radius, $r_{\text {rail }}$ & $0.25 \mathrm{~m}$ & \\
\hline Groove radius, $r_{\text {groove }}$ & $0.35 \mathrm{~m}$ & \\
\hline Wheels per rail, $N_{\text {wheel }}$ & & Considering polyurethane wheels \\
\hline Ball-chain rope diameter, $d_{r}$ & $0.016 \mathrm{~m}$ & \\
\hline Ball-chain top tension, $T_{t o p}$ & $18620 \mathrm{~N}$ & \\
\hline Ball-chain bottom tension, $T_{b o t}$ & $17911 \mathrm{~N}$ & \\
\hline Ball-chain max displacement, $y_{\max }$ & $1.94 \mathrm{~m}$ & \\
\hline Chemical agitation power, $P_{a g}$ & $12 \mathrm{~kW}$ & \\
\hline Chemical heating power, $P_{\text {heat }}$ & $9.96 \mathrm{~kW}$ & \\
\hline Chemical pumping power, $P_{\text {pump }}$ & $2.98 \mathrm{~kW}$ & \\
\hline Roller motorizing power, $P_{\text {roller }}$ & $117 \mathrm{~kW}$ & \\
\hline Total power required, $P_{\text {total }}$ & $172 \mathrm{~kW}$ & \\
\hline
\end{tabular}


(a)

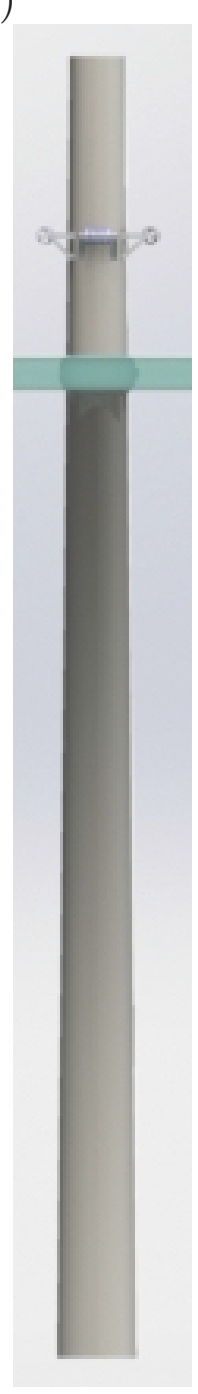

(b)

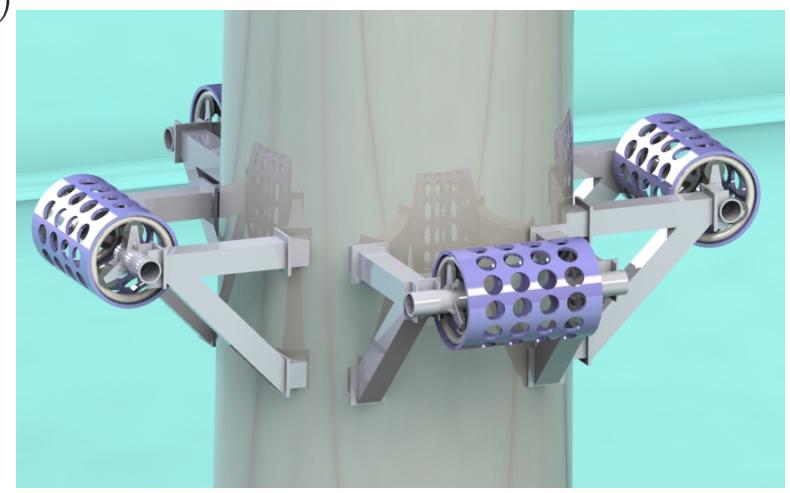

(c)

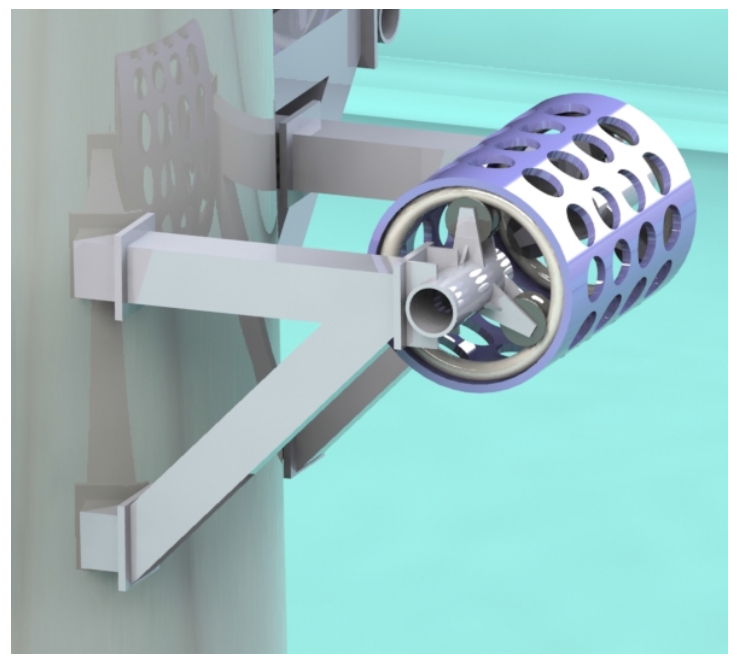

Figure 5-7: Three-dimensional model of SMORE for the a 1/10th physical scale version of the design case outlined in table 5.7 . The entire $1 / 10$ th scale system is show on the turbine spar in (a); whereas (b) shows a close-up of the upper platform, and (c) is a close up of one of the roller subsystems (shown in purple). 


\section{Chapter 6}

\section{SMORE 1/10th Physical Scale Prototype Ocean Test}

This chapter describes the design and ocean testing of a 1/10th physical scale prototype of the Symbiotic Machine for Ocean uRanium Extraction (SMORE). This research was done in collaboration with Dr. Ken Buesseler and Jessica Drysdale at the Woods Hole Oceanographic Institution. The results of this test will inform the design of a full-scale, symbiotic system to harvest uranium from seawater.

\section{$6.1 \quad 1 / 10$ th physical scale prototypes}

In the full-scale design, described in the previous chapter, large rollers are used to move ball-chain nets down the entire length of the turbine. One question that arose in the development of the design was whether the ball-chain nets should be continuously moving through the ocean or (i.e. should the rollers continuously cycle the ball-chain net like a conveyor belt through the ocean?) completely stationary. The additional movement of the ball-chain net might induce more seawater flow to the fiber adsorbents within the shells and hence increase the uranium adsorbed. However, the additional complexity of a continuously moving system as opposed to a stationary system would likely result in higher capital and operational costs for the machine. The question remains as to whether the value of the additional uranium adsorbed by a system continuously moving the adsorbent fibers would offset the increased cost of the system due to its increased complexity. For this reason, two modules were prototyped at a 1/10th physical scale for prolonged ocean testing: one system in which the net remained stationary and another in which the net was continuously moving. Figure $6-1]$ shows the main components of each of the two systems and their location on a wooden float for ocean testing.

\subsubsection{System 1: Stationary net system}

The stationary net system, shown in figure 6-2], comprised a roller and wheel assembly, surface support structure, motor and gearbox, power transmission using a timing belt, 


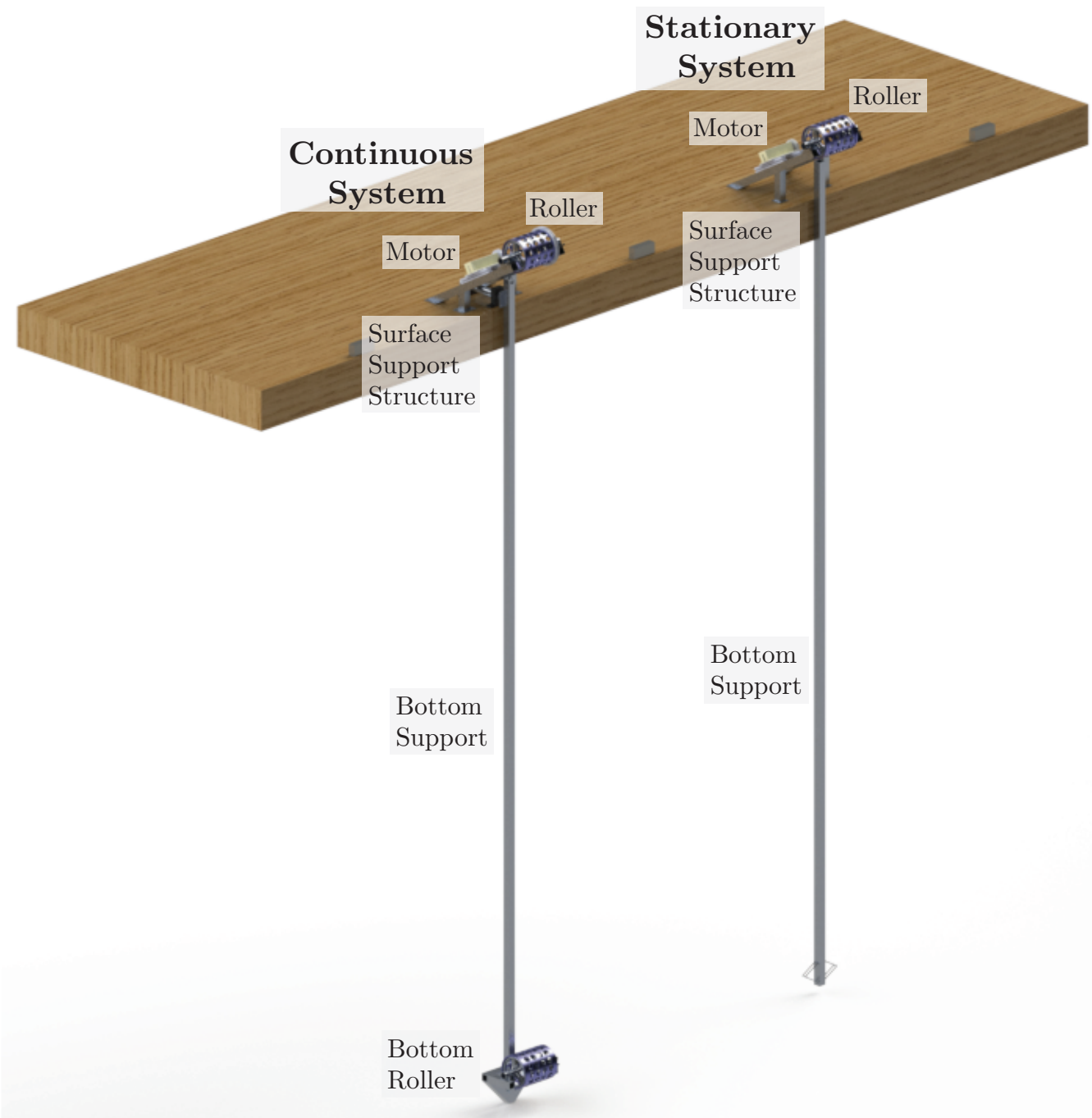

Figure 6-1: Three-dimensional model of 1/10th physical scale model for ocean testing of the SMORE design described in chapter 5. Both a stationary and continuous version of the design were fabricated and mounted to a wooden float for ocean testing.

battery, $18 \mathrm{ft}$ long bottom support structure, and two guide wires. The roller was constructed from a 8.625 in outer diameter pipe, 0.322 in thick, with a length of 11 in and 1.6 in diameter circular holes to fit the shells of the adsorbent net. The roller was designed to fit 13 shells along its circumference and four shells along its length.

The roller was motorized in order to wind and unwind the shell enclosure net periodically to retrieve adsorbent samples. This motorization was accomplished using grooved wheels on a circular track welded to the interior of the roller on either end (figure 6-2b). The number of grooved wheels required was determined by using the maximum pressure of the contact for an ellipsoid Hertz contact between the wheels and the groove, as well as the friction required to provide sufficient torque to move the roller. Using a similar analysis to that described in chapter 5 , it was found that three polyurethane wheels of approximately 1.77 in outer diameter along a 0.675 in 

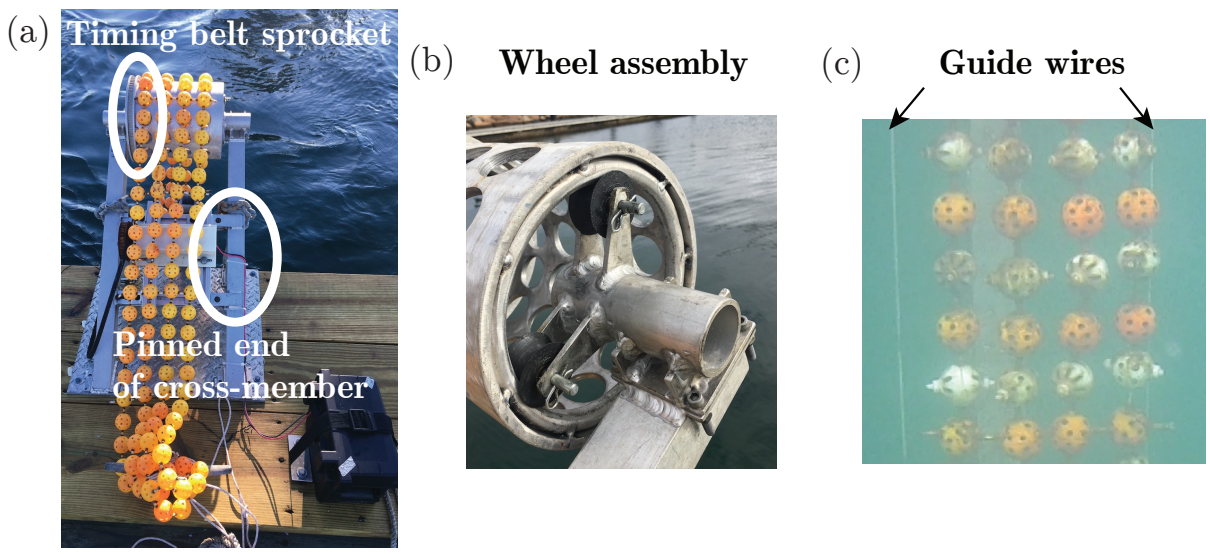

Figure 6-2: Stationary system design of (a) surface structure, (b) wheel assemblies, and (c) net and guide wires.

diameter aluminum rail would be sufficient.

A brushed DC motor with a torque rating of 345 oz-in was used to drive the roller. The motor was fitted with a 12:1 gearbox in order to reduce the speed. The power was transmitted using a timing belt from the motor to a fabricated sprocket welded to the exterior of the roller. The motor was powered using a $7 \mathrm{amp}$, rechargeable lead acid battery housed in a waterproof battery box mounted next to the surface structure of the stationary system.

A thrust bearing was used on one side of the top wheel on the belt side of the roller to counteract the force of the timing belt pulling the belt side of the roller and thereby causing the top wheel to fall out of alignment and possibly causing it to rub to one side resulting in premature failure due to friction. This bearing was able to take the radial load of the wheel pushing against the side support and push the wheel back towards the intended alignment. All other wheels would then move to compensate for the imperfections in the welded rails as needed, so as not to over-constrain the system.

The wheel assemblies were first connected to the roller and then bolted into surface support members. In order to ensure alignment between the roller and both wheel assemblies without over-constraining the system, the surface support structure was fabricated with a cross-member connecting the two sides. This cross-member was welded to one surface support and pinned to the other using carriage bolts through a thin attachment plate. These cross-members also served as a mount for the motor subassembly. The entire surface assembly was then bolted into an aluminum plate to maintain alignment and this aluminum plate was then bolted onto the wooden float.

To reduce the likelihood of the stationary adsorbent net experiencing entanglement or being dragged away by strong currents, it was clipped in place to galvanized vinylcoated wire rope that was attached on either side of the bottom support pole (figure 6-2 $c$ ). Given that the net was inherently buoyant, small dive weights were added to the bottom of the net to ensure it did not float. 


\subsubsection{System 2: Continuous net system}

Aside from the bottom support, net, and motor assembly, the continuous net system was analogous to the stationary net system. In the case of the continuous net system, the shell enclosure net was a complete loop, effectively making a conveyor belt. Originally, a roller, similar to the one on the surface, was designed to be attached to the bottom of the $18 \mathrm{ft}$ long bottom support.

As with the stationary system, a brushed DC motor with a torque rating of 345 ozin was used to drive the roller. However, the speed was reduced significantly using a 256:1 gearbox. The motor was powered by a $12 \mathrm{~V}$ AC to DC converter, rated for up to 25 amps. On average, the system drew approximately 8 amps, resulting in an output torque of approximately $15 \mathrm{oz}-\mathrm{in}$. The resulting linear velocity of the adsorbent net was approximately $4.7 \mathrm{in} / \mathrm{s}(\sim 12 \mathrm{~cm} / \mathrm{s})$.

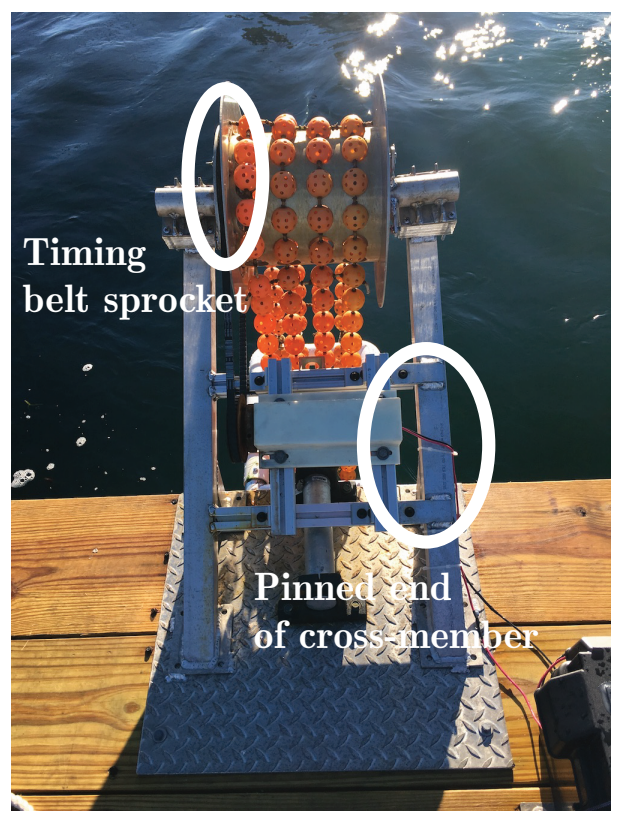

Figure 6-3: Fabricated surface structure of the continuous system design.

\subsection{Ocean test site}

The prototypes were tested at the Massachusetts Maritime Academy (MMA) in Buzzards Bay, MA. They were located at the end of the MMA dock where the water depth was approximately 23 feet at low-tide. Although fairly close to shore, the tides in this location vary up to 6 feet and the currents can be extremely strong due to the proximity to the Cape Cod Canal, which frequently has currents of about 5 knots $(2.6 \mathrm{~m} / \mathrm{s})$. Additionally, the wind often generates waves of approximately 1-2 feet in height at this location. For these reasons, this site was deemed to have conditions that could provide valuable results from the prolonged ocean testing of the 1/10th physical scale SMORE prototypes. 
(a)

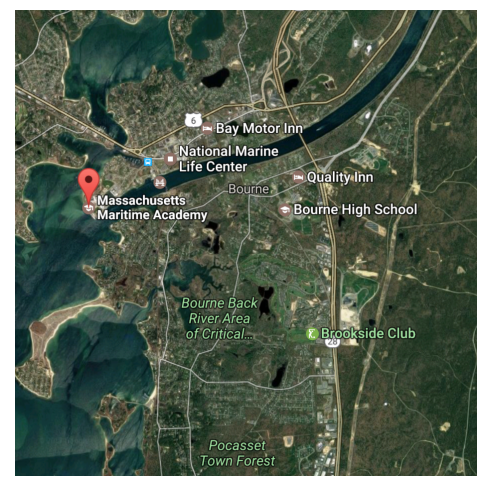

(b)

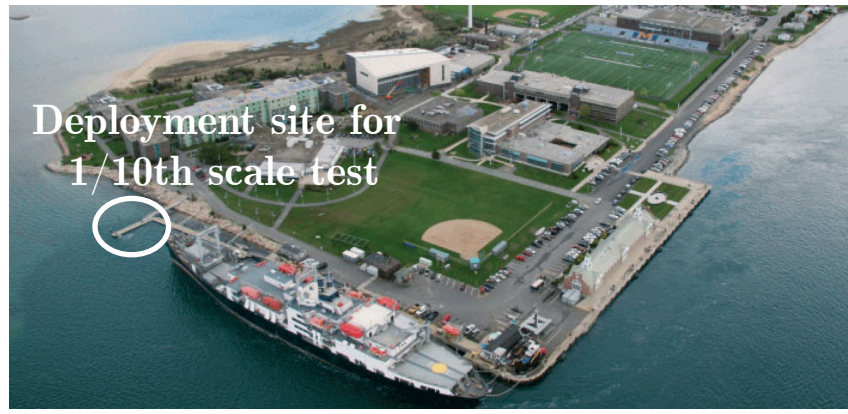

Figure 6-4: Massachusetts Maritime Academy (MMA) (a) location in reference to Cape Cod Canal and Buzzards Bay, and (b) aerial view of MMA's campus with the location of the prototype test indicated by the white circle.

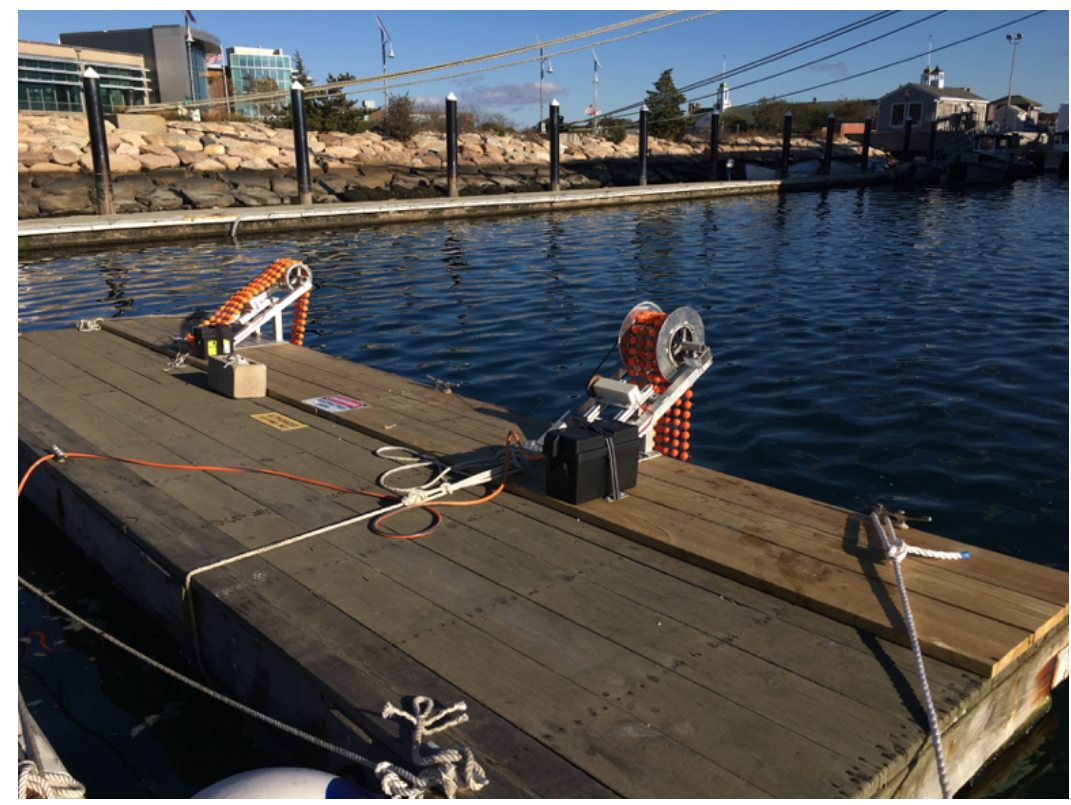

Figure 6-5: Both prototypes mounted to the wooden float and moored to the end of the MMA dock during testing.

Both systems were mounted to a single wooden float measuring $24 \mathrm{ft}$ long and 7 $\mathrm{ft}$ wide which was moored to the end of the MMA concrete dock. A variety of ocean sensors were also deployed along with the prototypes. These sensors were mounted at various depths along a piling located at the end of the concrete dock within $3 \mathrm{ft}$ of the wooden float, where the ocean depth was approximately $19 \mathrm{ft}$ at low tide.

\subsection{Shell enclosure net}

In the prototypes designed and tested in this chapter, the adsorbent shells were incrementally spaced along high strength mooring rope, and then strung together to 
(a)

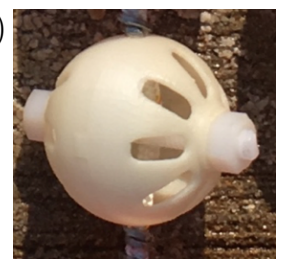

(b)

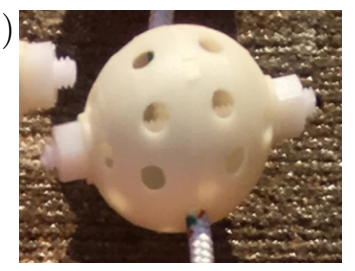

Figure 6-6: Shell designs for the testing of uranium adsorption fibers using (a) slotted holes and (b) circular holes.

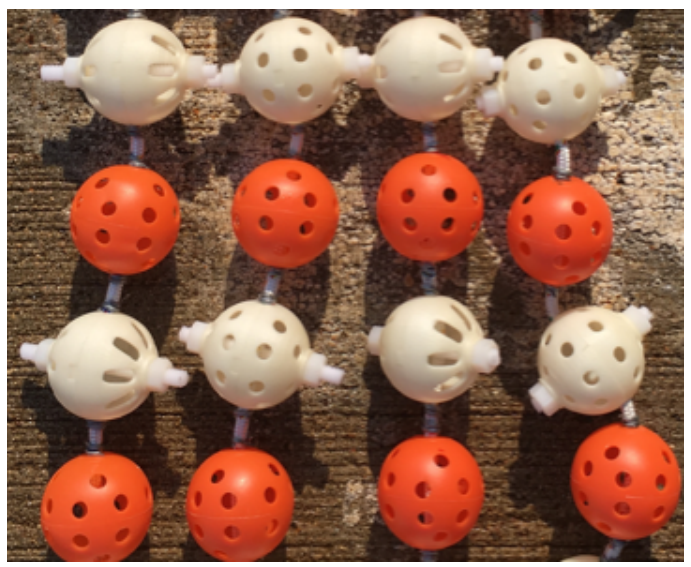

Figure 6-7: Small section of net with white shells for adsorbent fibers. Adsorbent enclosure shells were alternated with orange placeholder shells used for mechanical testing.

create a net using cross-members of the same rope to increase the net's rigidity and reduce the likelihood of tangling. Both prototypes used the same type of net, with four lengths of shells combined to make a single net. The vast majority of the shells for the ocean test did not contain uranium adsorbing fibers and were used primarily to test the mechanical components of the system. The net for the stationary system was approximately $20 \mathrm{ft}$ long and used a total of 430 shells. The net for the continuous system was about $42 \mathrm{ft}$ in length and required 960 shells.

The net was constructed by stringing placeholder 1.6 in diameter shells along $3 / 16$ in diameter rope. The rope comprised of a braided cover and a braided core made from high-performance aramid, also known as Technora, resulting in a high-temperature (rated up to $300^{\circ} \mathrm{F}$ ), wear-resistant rope meant for lifting (rated for $550 \mathrm{lbs}$ capacity). The shells were spaced approximately 0.45 in apart lengthwise such that 13 shells fit around the circumference of a 8.625 in diameter roller, and approximately 2.23 in apart widthwise, so that four lengths fit along the length of the 11 in roller. The shells were kept in place along the rope by 16 gauge steel c-ring staples.

To test the uranium uptake of the AI8 adsorbent fibers used on the prototypes, two shell designs were investigated, as shown in figure 6-6]. The first used slotted holes while the second used circular holes. Each net included nine of each type of design, for a total of 18 shells per net that would include AI8 fibers (see figure 6-7). The shells were 3D printed out of acrylonitrile butadiene styrene in two halves with a 
series of tabs and corresponding slots to ensure the two halves were aligned the same way each time they were connected. The two halves were kept together using a $1 / 4$ in threaded acetal rod placed through the middle and two nylon hex nuts on either end. On the stationary net, the shells with the adsorbent fiber were placed approximately $10 \mathrm{ft}$ below the ocean surface so that they were about mid-depth in the water column when deployed. In the case of the net for the continuously moving system, two narrow cable ties were also used to secure each half of the shells together to ensure the shells would not come apart unintentionally. The shells were made water-proof using three coats of polyurethane to reduce the amount of water absorbed by the shells which could cause unwanted deformation during the experiment.

\subsection{Adsorbent deployment and sampling}

As mentioned previously, each system contained 18 shells in which the uranium adsorbent was placed, for a total of 36 shells with adsorbent fibers. The adsorbent was deployed as a "mini braid" (figure 6-8), a pre-weighed, small mass (80-100 mg) of adsorbent fiber that was cut from a common AI8 adsorbent braid prepared by Oak Ridge National Laboratory. Each mini braid was weighed before deployment, after retrieval, and also after sample digestion. The weight after retrieval would include any amount of biofouling (the growth of organisms on the fiber), however it would not account for any loss of fiber occurring during the deployment. The weight after sample digestion would allow for the determination of adsorption capacity as a function of the adsorbent mass.

The stationary system included two mini braids per shell while the continuous system had about one to two mini braids per shell. Additionally, mini braids were included in two nylon mesh bags at the bottom of the stationary net to act as controls (i.e. to determine if the shells significantly inhibited the uptake of uranium due to the shell's obstruction of seawater flow). The first adsorbent sample was collected 24 hours after deployment. The eight subsequent samples were collected every seven days

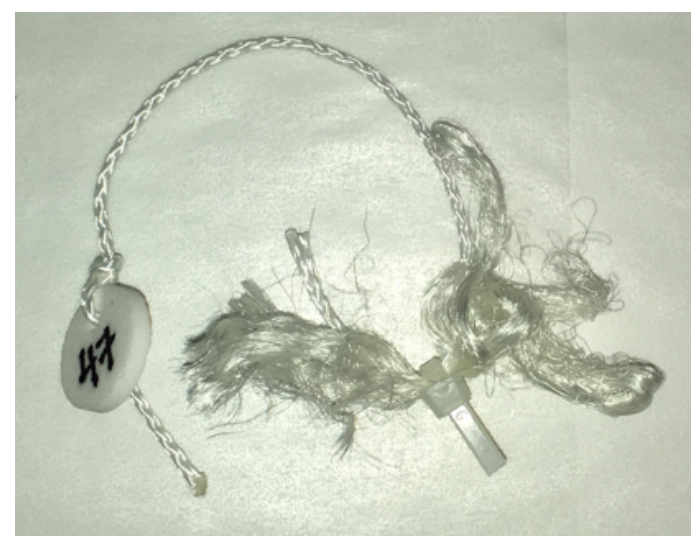

Figure 6-8: Pre-weighed adsorbent mini braid. One to two of these mini braids were placed in each shell design on each of the two prototypes. 
after deployment. This sampling resulted in a determination of uranium adsorption as a function of time for each system, each shell design, as well as the control bags. Water samples were also collected on each of the nine sample dates for trace metal analysis.

\subsection{Ocean test measurements}

Along with the mechanical testing of the physical prototype, sensors were placed throughout the water column to measure physical properties of the ocean that directly affected the uranium adsorption rates of the fibers during the deployment. Most of the sensors were mounted at various depths along a piling at the end of the dock at MMA, within $3 \mathrm{ft}$ of the wooden float with the prototypes. All sensors placed on the piling were deployed on August 10, 2016 and retrieved on December 18, 2016. In addition to the sensors on the piling, a Xylem EXO-2 Sonde measured salinity as part of a water quality assessment of Buzzards Bay from October 4, 2016 to December 13, 2016. The location of the prototype float test, the pilings, and the Xylem sensor are shown in figure 6-9. Figure 6-10 shows the distribution of sensors along the piling.

As described in section [2.5.2, temperature has been shown to have strong implications of the adsorbent's uranium uptake. For the case of the AI8 fiber, equation $(2.2)$ becomes $(2.15)$ :

$$
y=\frac{(0.3775 T-0.2077) t}{(0.4083 T+14.13)+t}
$$

where $T$ is the temperature, ${ }^{\circ} \mathrm{C}$ and $y$ is the resulting uptake of uranium $(\mathrm{g} \mathrm{U} / \mathrm{kg}$ adsorbent). Figure $\mathrm{b}-\mathrm{C}$ shows the resulting time-dependent uptake for the AI8 adsorbent for $\mathrm{T}=10^{\circ} \mathrm{C}, 15^{\circ} \mathrm{C}$ and $20^{\circ} \mathrm{C}$ as predicted by (6.]). As can be seen from the figure, a difference of just $5^{\circ}$ in temperature results in an over $50 \%$ increase in the uranium uptake.

Temperature was measured throughout the water column as well as on the two prototypes using ONSET Tidbit Water Temperature Loggers. One Tidbit, sampling in 30 second intervals, was mounted on the continuously moving net below the section of the white shells containing adsorbent. Another, sampling at 5 minute intervals, was mounted in a similar location on the stationary system, and a third, sampling at 5 minute intervals, was placed in one of the nylon mesh bags at the bottom of the stationary system.

As described in chapter 2 , salinity has also shown to have a large impact on the adsorbent's uranium uptake as it directly indicates the amount of uranium present in the water by a well-defined relationship between ${ }^{238} \mathrm{U}$ concentration in seawater and

salinity where the concentration of ${ }^{238} \mathrm{U}$ in $\left(\mathrm{ng} \mathrm{g}^{-1}\right)$ shown by (Owens et al. (201]) to be described as

$$
{ }^{238} \mathrm{U}( \pm 0.061)=0.100 \times \mathrm{S}-0.326
$$

where $\mathrm{S}$ is the salinity. 


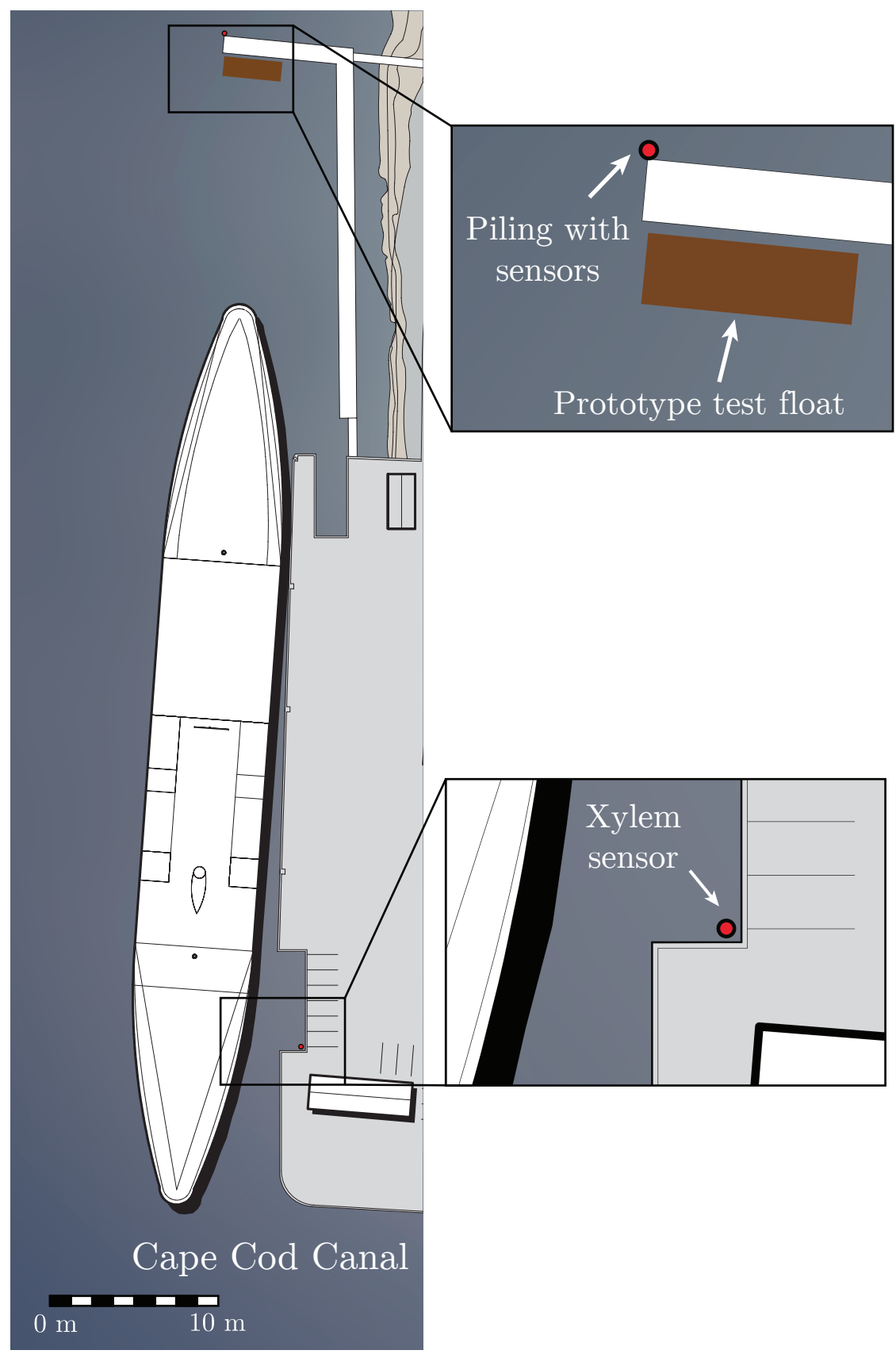

Figure 6-9: Map of MMA indicating the location of the wooden float with the prototypes, the piling to which most of the ocean sensors were attached, and the Xylem sensor. Note: the pier to which the Xylem sensor was located is fixed, not floating, on pilings and has water free flowing under it. The spot that the Xylem sensor was located was not protected by the pier and ship as the bird's eye view suggests.

Hence, the salinity was measured at mid-depth in the water column using a HOBO U24 Conductivity Logger (which also measured temperature), sampling at 15 minute intervals. It was also measured by the Xylem EXO-2 Sonde, mounted $3 \mathrm{ft}$ above the seafloor, sampling at 15 minute intervals. 


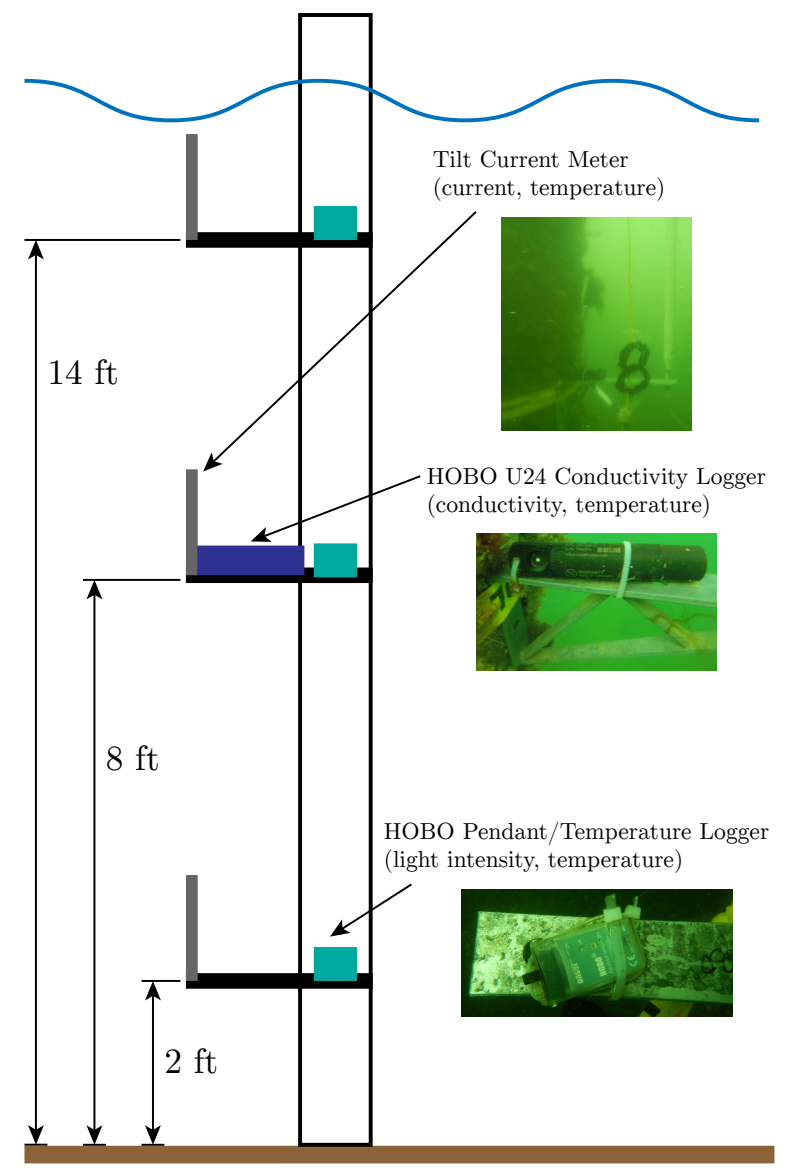

Figure 6-10: Layout of instruments attached to piling at the end of the dock.

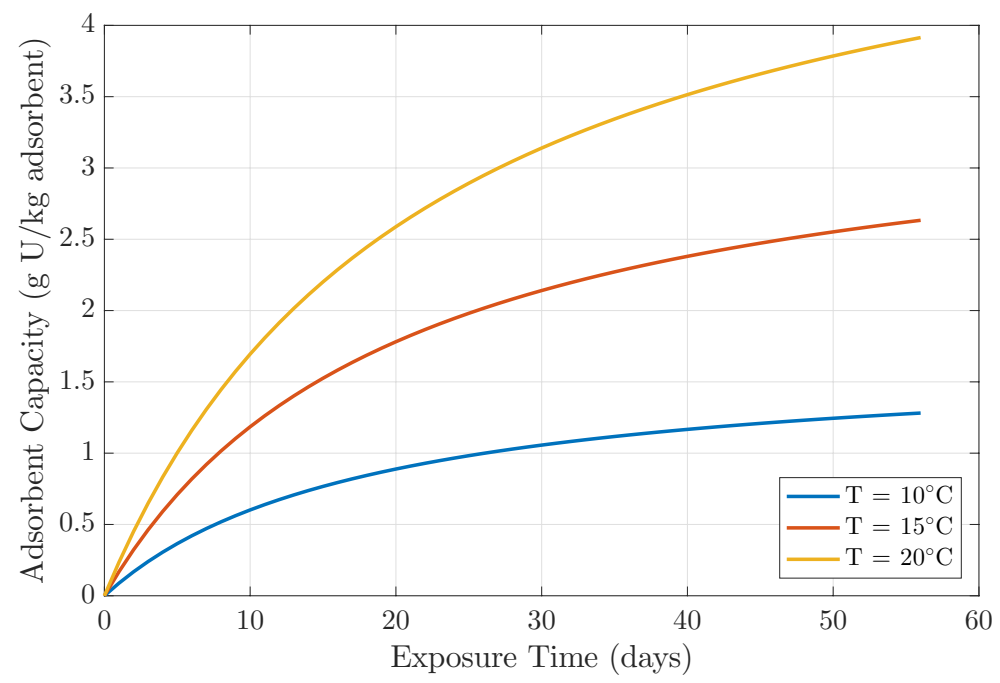

Figure 6-11: Time-dependent uptake of uranium for the AI8 adsorbent fiber as predicted by 6.0 for $\mathrm{T}=10^{\circ} \mathrm{C}$ (blue line), $15^{\circ} \mathrm{C}$ (red line) and $20^{\circ} \mathrm{C}$ (yellow line).

As described in section 2.5.3, the amount of light has been shown to have strong effects on the biofouling of the adsorbent and the related uranium uptake. In order 
to gather quantifiable data related to biofouling, light was measured at three depths using HOBO Pendant Light/Temperature Loggers (which also measure temperature), sampling at 10 minute intervals.

Previous work has shown that the uranium adsorbed by the adsorbent fibers is also highly dependent on the flow rate of the water, as described in detail in section 2.5 .4 . In order to gather information regarding the water flow seen by the two prototypes, current was measured using Tilt Current Meters from Lowell Instruments, placed at three depths. The current meters also measured temperature and were programmed to sample every second.

\subsection{Results}

The prototypes were deployed for a total of 56-days, starting on October 18, 2016 and ending on December 13, 2016. The first adsorbent sample was taken 24 hours after deployment on October 20, 2016 and the ninth and final sample was completed on day 56. The results described below include insights gained from the mechanical testing of the system, comparison between the stationary and moving systems, as well as the physical properties and changes over time of the seawater at the test site itself.

\subsubsection{Continuous system modifications}

In the original the continuous net system design, the adsorbent net was placed in tension between the roller above the ocean surface and one attached to the bottom support structure, such that the adsorbent net transmitted the movement from the upper roller to the submerged roller. Ensuring there was enough tension in the system in order to accomplish this proved to be difficult as the currents could be extremely strong and resulted in either causing the adsorbent net to slip off the bottom roller (figure $6-12 a$ ), or, after additional guides were welded on to the ends of the bottom roller, to move out of alignment on the roller (figure $6-12 b$ ).

Because providing enough tension was difficult, the moving bottom roller was replaced with a stationary 4 in diameter PVC pipe. This allowed for the adsorbent net

(a)

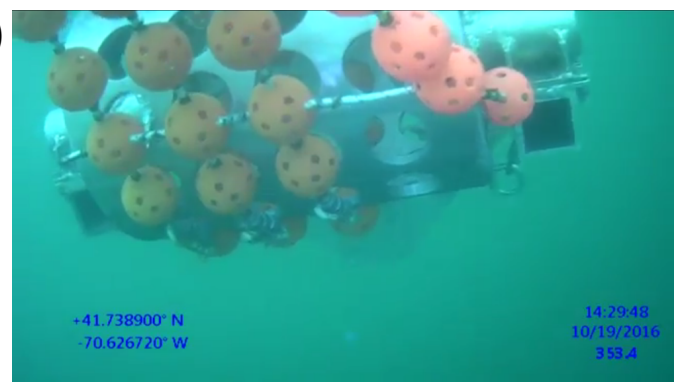

(b)

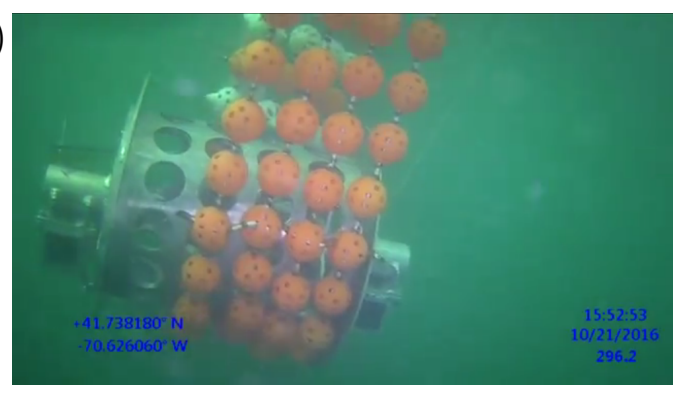

Figure 6-12: Issues with the bottom roller that arose due to the inability to provide enough tension in the adsorbent net for prolonged periods of time. The adsorbent net was found to (a) slip off or (b) fall out of alignment with the roller. 
(a)

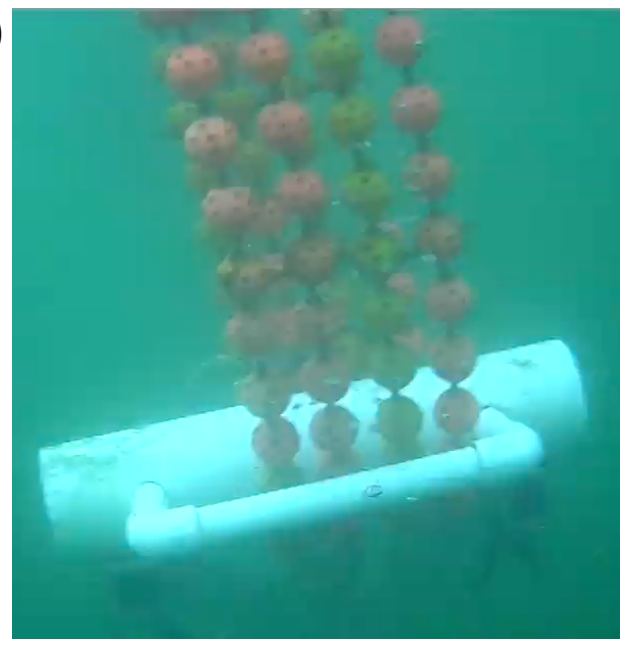

(b)

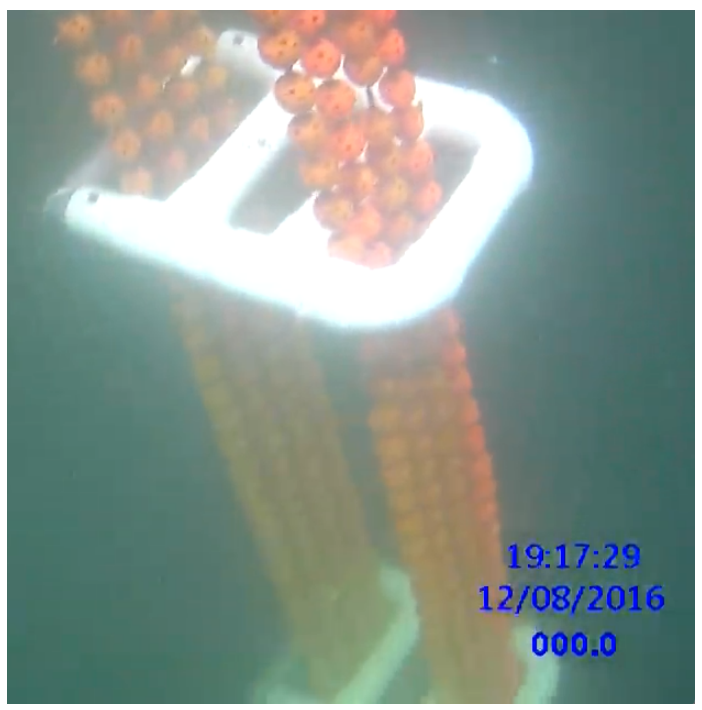

Figure 6-13: Major modifications made to the continuous system consisted of a (a) stationary 4 in diameter PVC pipe to replace the moving bottom roller and (b) a series of PVC guides along the length of the bottom support to keep the net separated and prevent tangling and misalignment due to strong currents.

to easily slide over the pipe and be completely driven by the upper roller. Additionally, to prevent movement and tangling between the two sides of the net due to the strong currents, guides that separated the two sides of the net were added at four points along the length of the bottom support. The guides also ensured good alignment between the net and the upper roller as it exited the water.

These complications and design modifications resulted in intermittent operation of the continuous system. In total, the continuous system was moving for approximately $37 \%$ of the total deployment, mostly towards the end of the experiment, with the longest three operations being 5.9 days, 5 days, and 4.7 days. Failures toward the end of the deployment were due to ice build up caused by the dropping temperatures off the coast of Massachusetts with the onset of winter.

\subsubsection{Sensor data}

With the exception of the failure of the Tidbit temperature sensor located with the shells of the stationary net, the premature battery failure of the current meters (which stopped logging at approximately day 25 of the 56-day deployment), and an issue with the U24 meter's conductivity readings, data was collected from all sensors throughout the entirety of the experiment.

As expected, the light intensity dropped off exponentially with depth (figure 614la). The exponential drop off is most pronounced in the beginning of September (with a difference of about $88 \%$ in the light intensity between the upper and lower light sensors) and is least pronounced in December (with a difference of about $59 \%$ between the upper and lower light sensors). This reduction in light attenuation was likely due to less plankton and biota in the water in the fall and winter than the 

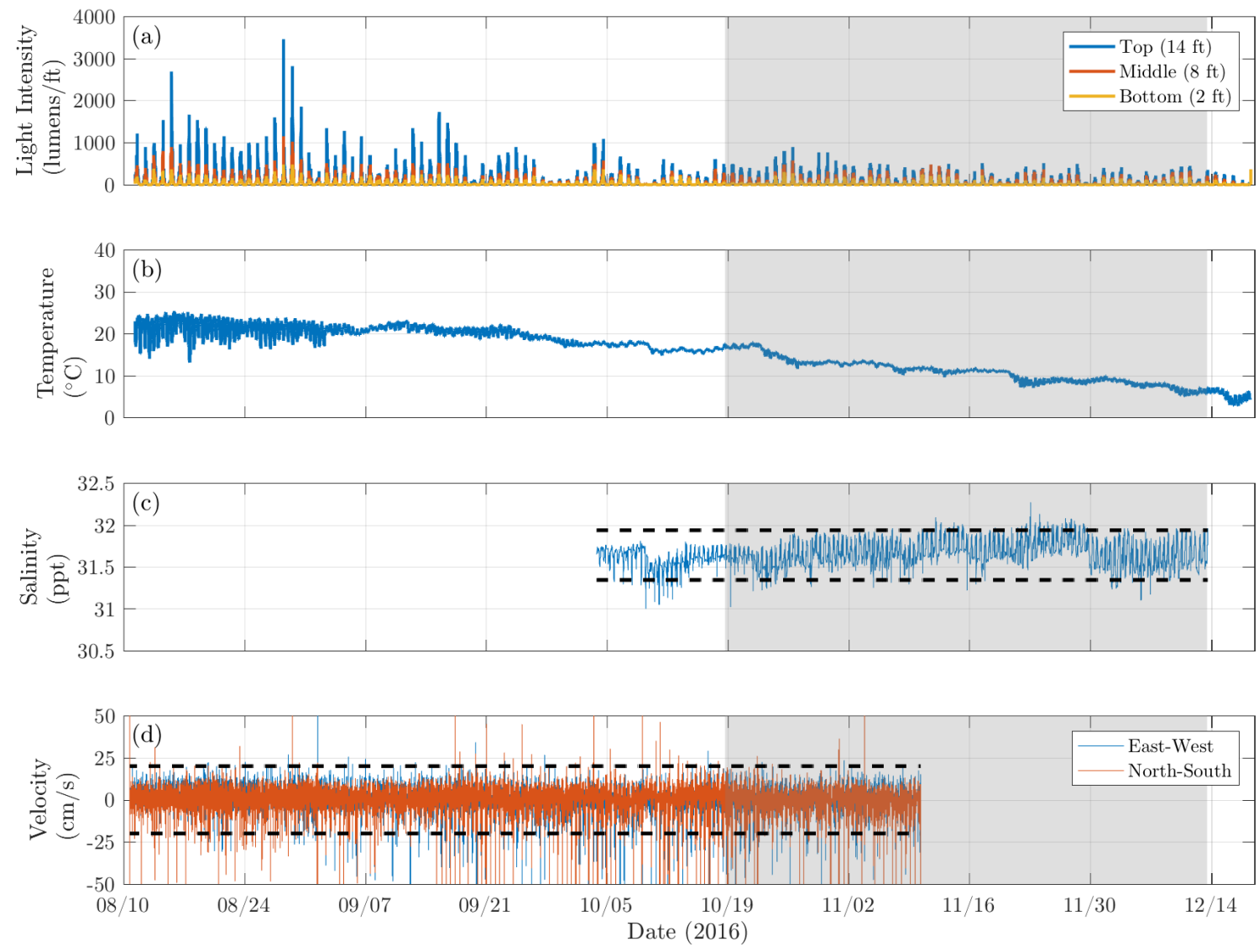

Figure 6-14: (a) Light intensity as measured by the top, middle, and bottom light sensors. (b) Temperature as measured from the U24 conductivity logger. (c) Salinity as measured from the Xylem EXO-2 Sonde salinity meter with dashed lines indicating $\pm 2 \hat{\sigma}$ where $\hat{\sigma}$ is the robust standard deviation. (d) Current as measured from the bottom current meter with the dashed lines indicating $\pm 20 \mathrm{~cm} / \mathrm{s}$. The gray rectangle indicates the period of the ocean test, October 20, 2016 - December 13, 2016.

summer.

Similarly, seasonal variations could also be seen in the temperature data as shown in figure 6-14b (note that there was excellent agreement in the temperature measured by all instruments so only the data from the conductivity logger is shown). The short term temperature differences were linked to the tidal variations at the site, with these variations becoming less pronounced over time. From equation ([.]), it is clear that temperature has a major affect on the uranium uptake. For instance, at a temperature of $18^{\circ} \mathrm{C}$, as seen close to the start of the 56-day deployment, by (6.]), the adsorbent capacity is estimated to be approximately $3.47 \mathrm{~g}-\mathrm{U} / \mathrm{kg}$-ads after 56 days. On the other hand, at a temperature of $5^{\circ} \mathrm{C}$, as seen close to the end of the 56-day deployment, the capacity is predicted to be decreased by $83 \%$ to approximately 0.60 $\mathrm{g}$-U/kg-ads. Given that the incremental adsorption of uranium decreases over time, it is likely that the colder temperatures could have a minimal affect on the uptake since they occurred toward the end of the deployment. 
The salinity of the ocean test side, shown in figure 6-14k, also varied with tides. Overall, the salinity remained between 31 ppt and 32.5 ppt, with no clear seasonal pattern.

Unfortunately, due to premature battery failure of the current meters from an error in programming upon deployment, the current data does not span the entirety of the deployment. As can be seen from figure 6-14d, which shows currents measured from the instrument near the bottom of the piling (approximately $2 \mathrm{ft}$ from the seafloor), while there were large short term variations in the currents (likely due to tides), overall there were little seasonal changes in the currents. In general, the currents were approximately $10-15 \mathrm{~cm} / \mathrm{s}$, with stronger periods of over $80 \mathrm{~cm} / \mathrm{s}$ at times. Note that the current meters saturate at approximately $\pm 80 \mathrm{~cm} / \mathrm{s}$ and therefore readings beyond this are not reliable. The currents seen at this ocean site are much larger than those tested in a lab setting which, to date, have been tested in flow rates of up to $8.24 \mathrm{~cm} / \mathrm{s}$ (Ladshaw et al, [2017).

\subsubsection{Biofouling}

As detailed in section [2.5.3, biofouling of the adsorbent fibers can have a detrimental affect on their ability to uptake uranium (Park et al, 2016). One striking result between the two systems was the difference in biofouling. At the end of the 56-day ocean test, the stationary system had a significantly higher amount of biofouling on its shells than the continuously moving system (figure 6-15). Biofouling begins with a thin biofilm and microorganisms followed by the attachment of larger organisms.

To further investigate if the reduced biofouling of the outer shells on the continuous system translated to reduced biofouling on the adsorbent fibers, the weight of the fibers after deployment (which would account for any organism growth) was compared to the weight of the fibers measured before deployment. Figure 6-16 details the percent weight gained or lost by the adsorbent fibers before and after deployment. The results show that in general, for all enclosures and systems, there was a trend toward weight loss in the beginning of the deployed and weight gain by the end of the deployment. The initial weight loss was likely due to the harsh marine environment shedding away some of the adsorbent fibers. On the other hand, the weight gain seen was likely due to biofouling and growth on the adsorbent fibers. However, there was little variation between the weight gained or lost between the different designs or systems.

This point is further illustrated by figure 6- -17 which shows the average percent weight gained or lost of the adsorbent fibers in each enclosure and system. As can be seen in the figure, the large standard deviation for all the enclosures suggests there was no significant difference in the weight gained or lost between the different enclosure and system types. This may have been due to the fact that the continuous system was only up for $37 \%$ of the deployment, with the majority of its uptime occurring at the end of the 56-day deployment. Considering that biofouling occurs within days to weeks of the adsorbent being placed in the ocean, it is likely that both systems saw about the same colonization of organisms given that the continuous system was not moving in the first few weeks of the ocean trial. Additionally, when the system 
(a)

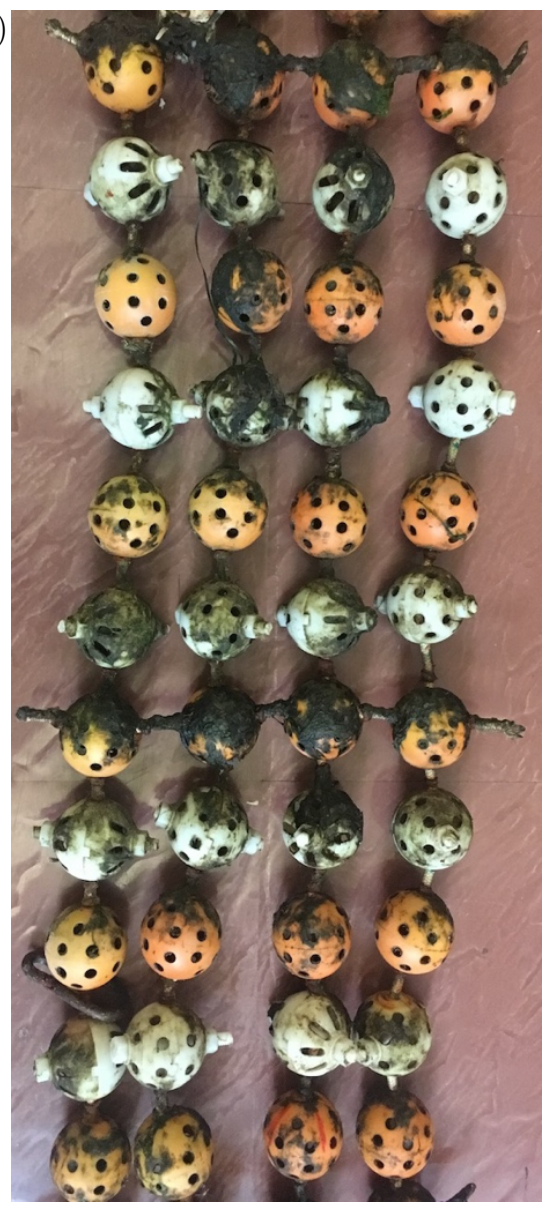

(b)

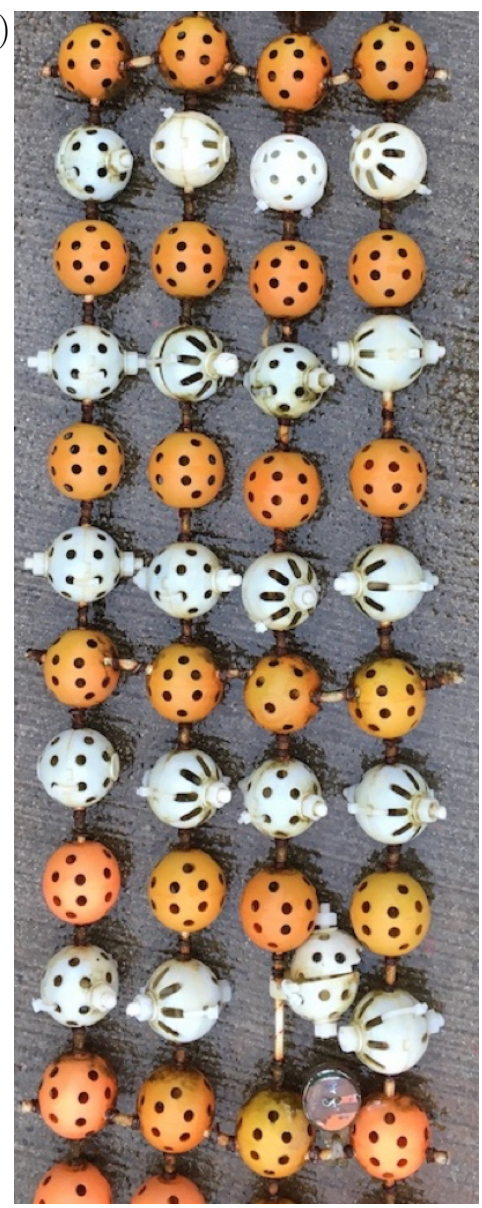

Figure 6-15: Biofouling on the (a) stationary net and (b) continuously moving net at the end of the ocean test.

was moving, the colder water temperature likely inhibited biofouling for all fibers, regardless of their enclosure or system.

\subsubsection{Water flow rate}

One of the objectives in the ocean test of the two prototypes was to determine if increased water flow could be achieved by continuously moving ball-chain through the ocean and if that translated to an increase in uranium uptake of the fibers enclosed by the shells on the net. To determine the answer, a novel method using the collection and measurement of radium extracted onto $\mathrm{Mn}-\mathrm{O}_{2}$ impregnated acrylic fibers was developed to quantify the volume of water passing through the fibers. This method was then utilized to quantify the water flow through each of the different types of enclosures, namely the control nylon mesh bag, the two shell designs on each of the two systems.

Preparation of the $\mathrm{MnO}_{2}$ impregnated acrylic fiber involved a 20 min immersion of raw acrylic fiber into a saturated $\mathrm{KMnO}_{4}$ solution heated to $75^{\circ}$ until the fiber turned black. At this point it was removed and rinsed throughly using deionized 
water that was previously filtered through $\mathrm{MnO}_{2}$ impregnated acrylic fibers to remove any radium. The fibers are then stored in damp plastic bags until they are ready to be used. (Moore, 1976).

The $\mathrm{MnO}_{2}$ impregnated acrylic fibers, which adsorb radium, were placed in a control cartridge, and each of the different types of enclosures. Figure 6-18la shows one of these fibers and figure $6-18 b$ shows the control cartridge used in this experiment. The fibers in each of the different enclosures were in the ocean for approximately 6.5 hours. At the same time, seawater from the same location was pumped up from about mid-depth in the water column to fill a large container in which the control

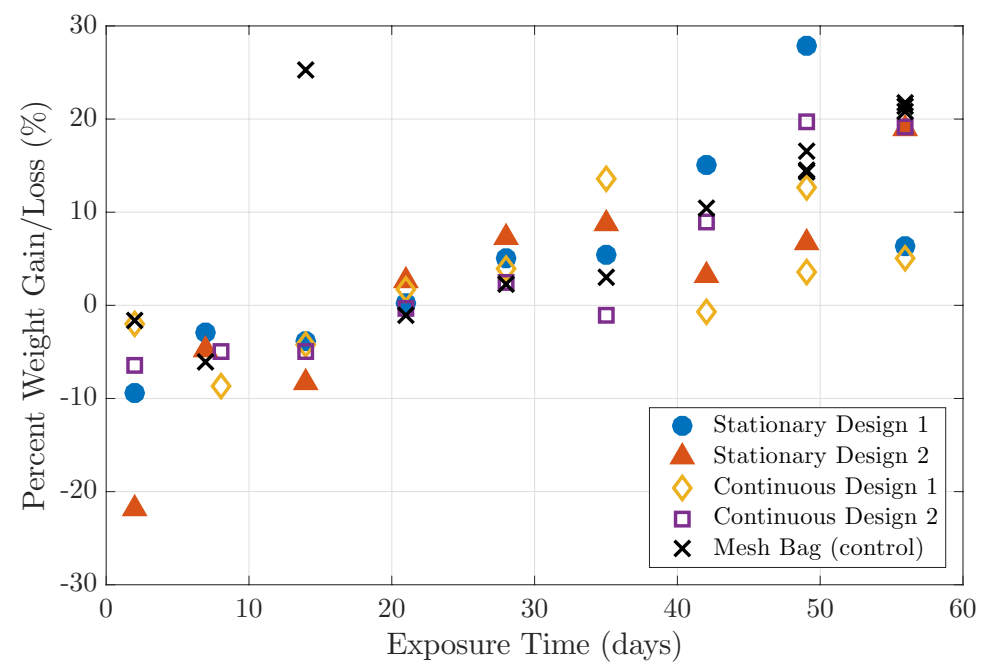

Figure 6-16: Percent weight gain or loss in the adsorbent fibers before and after deployment at each sampling.

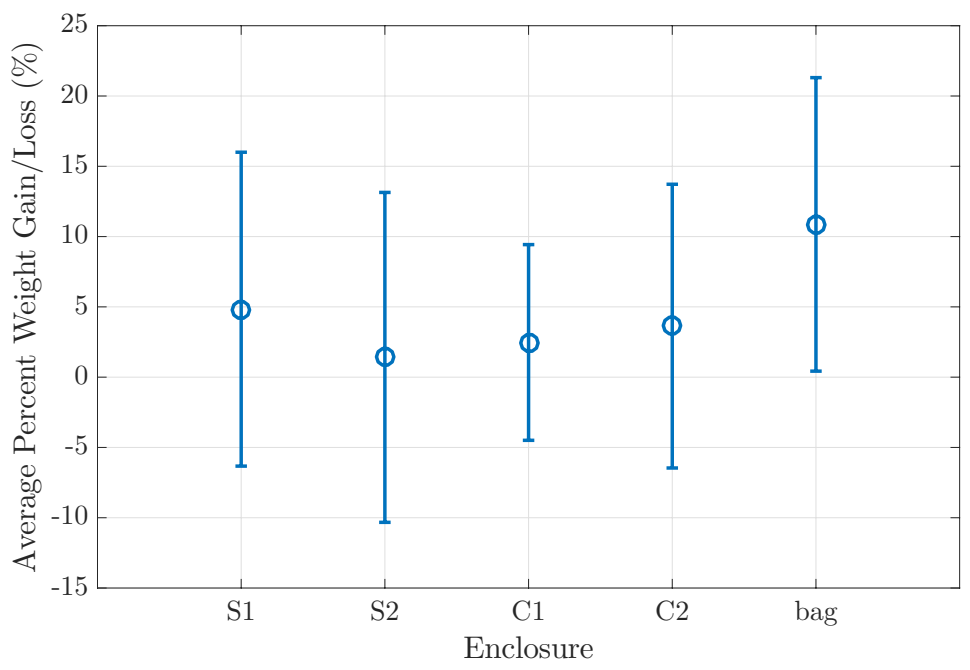

Figure 6-17: Average percent weight gain or loss in the adsorbent fibers for each enclosure and system of all the time-dependent measurements with error bars indicating the standard deviation. 
(a)

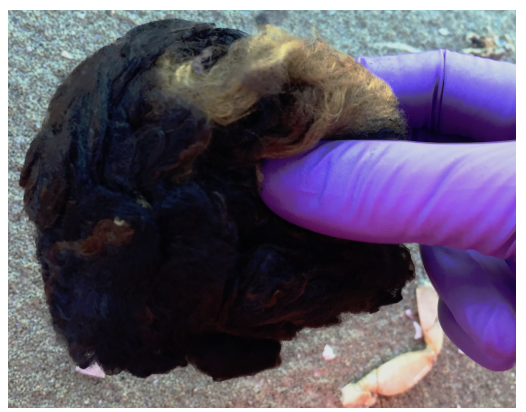

(b)

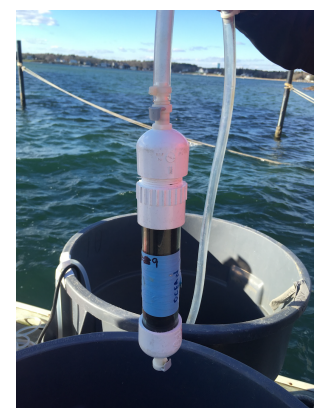

Figure 6-18: (a) $\mathrm{MnO}_{2}$ impregnated acrylic fibers used in this study and (b) the control cartridge housing some of these fibers through which a known volume of water was filtered.

(a)

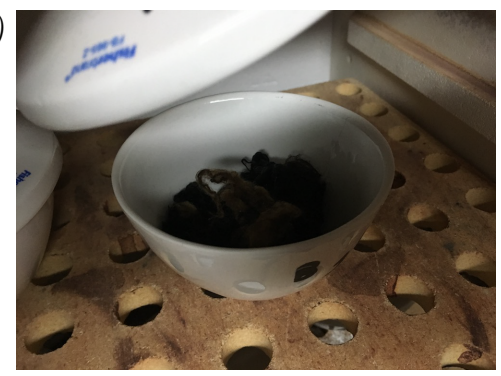

(b)

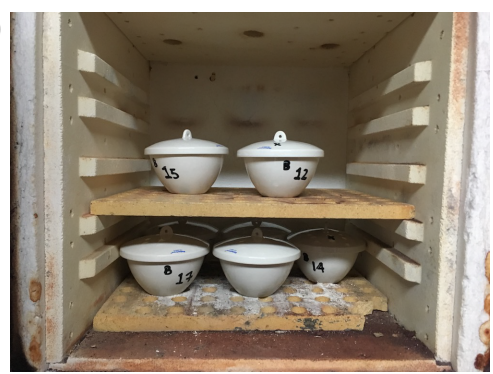

Figure 6-19: The ashing process showing (a) one clump of $\mathrm{MnO}_{2}$ impregnated acrylic fiber in a container ready for ashing and (b) all samples in a muffle furnace prior to ashing.

cartridge was placed. A known volume of the water in this container was slowly pumped through the control cartridge at about 1-2 L/min (a rate below $2 \mathrm{~L} / \mathrm{min}$ has been shown to achieve quantitative radium adsorption (Moore, 1976; Moore et al, 1995)). At the end of the 6.5 hour seawater exposure for all the test enclosures, the water still remaining in the large container to be pumped through the control cartridge was collected in smaller containers and processed at a later time.

After the seawater exposure, following the method of Moore (1984) the fibers were ashed in a muffle furnace at $820^{\circ} \mathrm{C}$ for 24 hours, resulting in a mass reduction of up to $60 \%$ (this step is shown in figure 6-19). After a three-week waiting period, which allows all daughters of ${ }^{226} \mathrm{Ra}$ to grow into equilibrium, the samples were counted for ${ }^{226} \mathrm{Ra}$ using $\gamma$-spectrometry by its photopeak at $352 \mathrm{keV}$.

The known volume of water filtered through the cartridge and the amount of radium adsorbed by the fiber in the control cartridge can be used to determine a relationship between the radium adsorbed and the water flow seen by the fiber. Figure 6-20] shows the results determined using this method after correcting for the differences in the amount of radium fibers initially placed in each of the enclosures and adjusting for weight due to ash loss. The results indicate that there was a significant difference in the water seen by the enclosures on the different systems. In particular, the shells on the continuous system saw the most amount of water flow, whereas the shells on the stationary system saw the least amount. In particular, the continuous system 


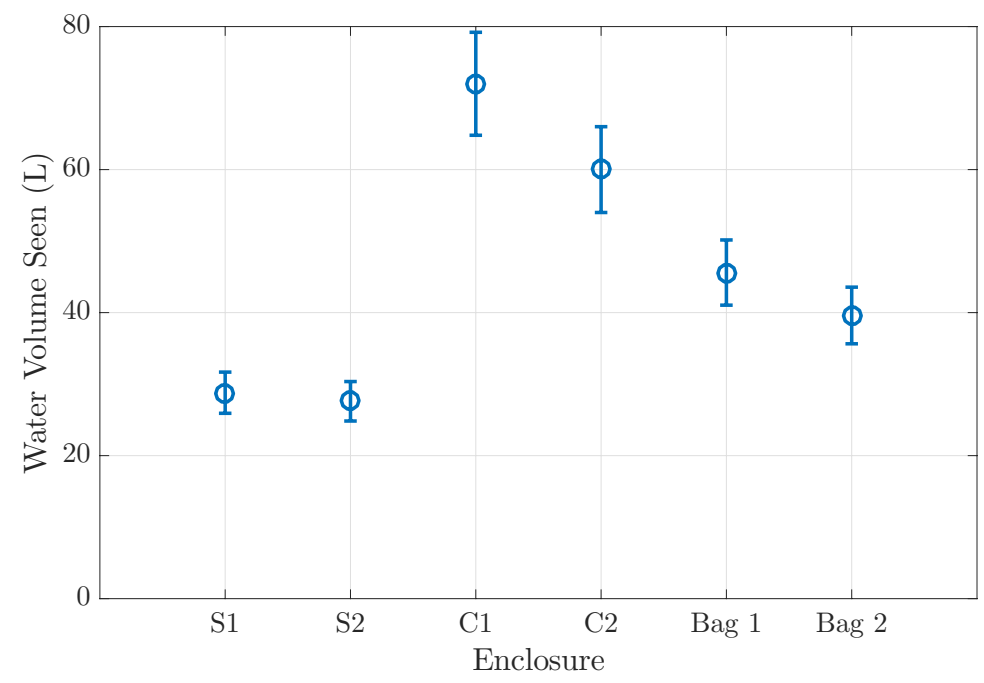

Figure 6-20: Volume of water seen by $\mathrm{MnO}_{2}$ impregnated acrylic fibers in different enclosure types on different prototype systems as determined by ${ }^{226} \mathrm{Ra}$ count using $\gamma$-spectrometry. The control cartridge saw $120 \mathrm{~L}$.

shells saw approximately $57 \%$ more water than the shells on the stationary system and $35 \%$ more water than the nylon mesh bags.

However, as indicated by the error bars in figure 6-20, there was no significant difference in the water flow seen by enclosures on the same system. That is, there was no difference in the water seen between the slotted and circular hole designs on the continuous system. As in the flume experiment, these results suggest that the actual design of the shell enclosure has little affect on amount of water flow to the interior. However, there was a significant difference in the water flow seen by the shell enclosures and the mesh bags on the stationary system. Though neither of these types of enclosures was moving, the mesh bags saw $33.8 \%$ more water flow than either of the shell designs. The results from the uranium adsorbed from the fibers on the stationary system from the mesh bag and the different shell designs will inform whether or not this increased water flow resulted in more uranium adsorbed, as was hypothesized.

\subsubsection{Uranium uptake}

Due to the conservative behavior of uranium in seawater, (№t et al., 2012) all uranium adsorption capacity data was normalized to a salinity of $35 \mathrm{psu}$ to correct for the varying salinity of natural seawater observed in different adsorption experiments. Adsorption kinetics and saturation capacity were determined by fitting time-dependent measurements of adsorption capacity using a one-site ligand saturation model described by (2.2). Figure 6-2] shows the time-dependent measurements of adsorption capacities for all trace metals retained by the AI8 adsorbent for each enclosure. As can be seen in the figure, uranium is not the dominant metal adsorbed by the fiber.

The uranium adsorption capacity ( $\mathrm{g} \mathrm{U} / \mathrm{kg}$ adsorbent) for all adsorbents in all the 

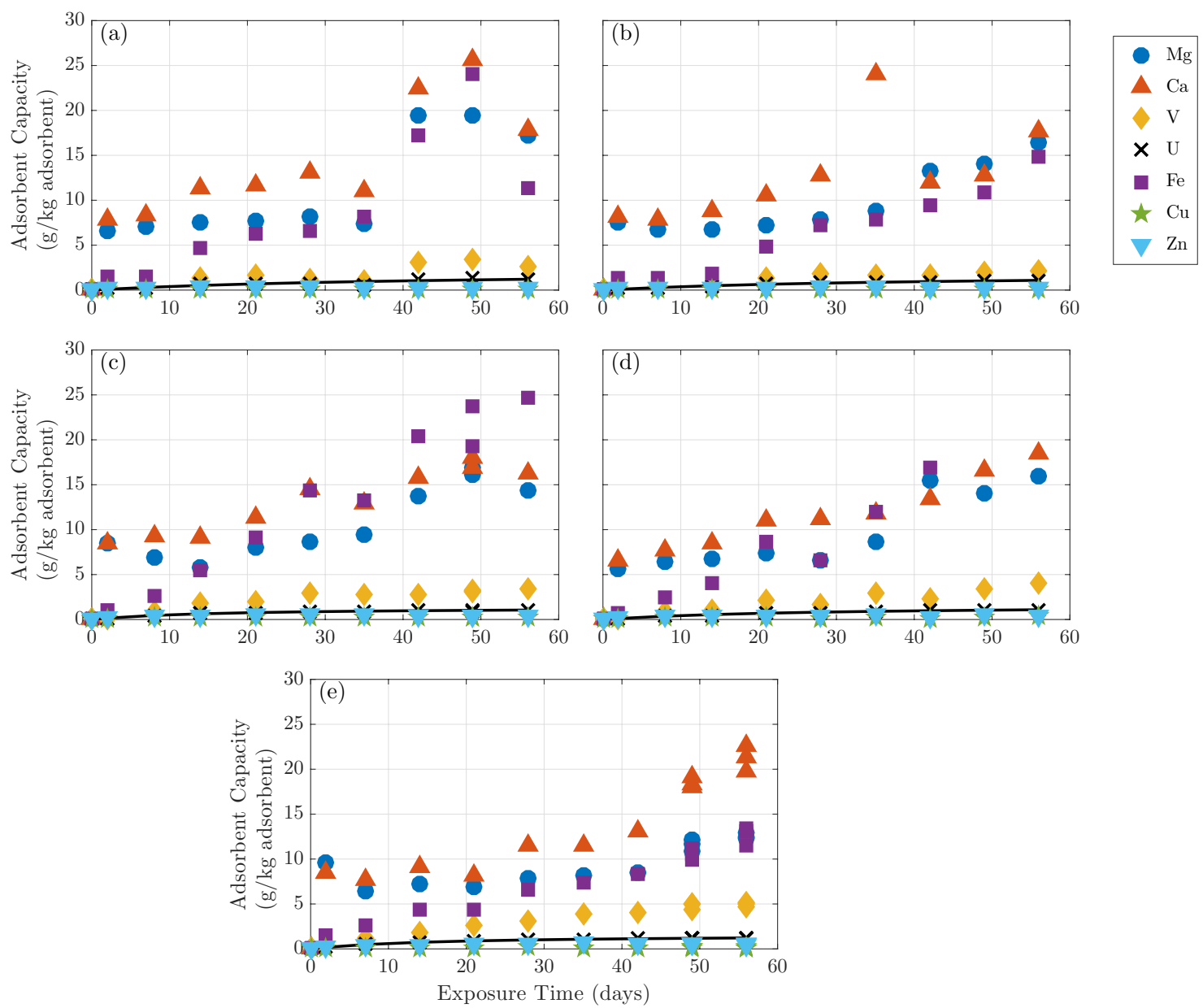

Figure 6-21: Time-dependent measurements of adsorption capacities (g element $/ \mathrm{kg}$ adsorbent) for several trace elements retained by the ORNL AI8 adsorbent used in the ocean test and enclosed in (a) shells with slotted and (b) circular holes of the stationary system, (c) shells with slotted and (d) circular holes of the continuous system, and (e) in the mesh bag on the stationary system. The black line drawn through the uranium adsorption data represents fitting to a one-site ligand model by $(2.2)$

enclosures and systems is shown in figure 6-22, with the lines indicating the one-site ligand saturation model fits for each enclosure. As can be seen, there is very little difference in the uranium adsorbed between the different enclosure or system types. This is further confirmed by figure 6-23 which shows the saturation capacity, $\beta_{\max }$, and half saturation time, $K_{D}$, as well as the uptake predicted for an immersion time of 56 days as predicted from the one-site ligand modeling. The error bars detail the $\pm 95 \%$ confidence intervals of the coefficients. As shown in the figure, there is no significant difference between the coefficients of the fits for each enclosure and system type, suggesting no significant difference in the uranium uptake by the adsorbent in different enclosures and on different systems. This suggests that the system movement, although increasing water flow to the adsorbent and decreasing biofouling on 


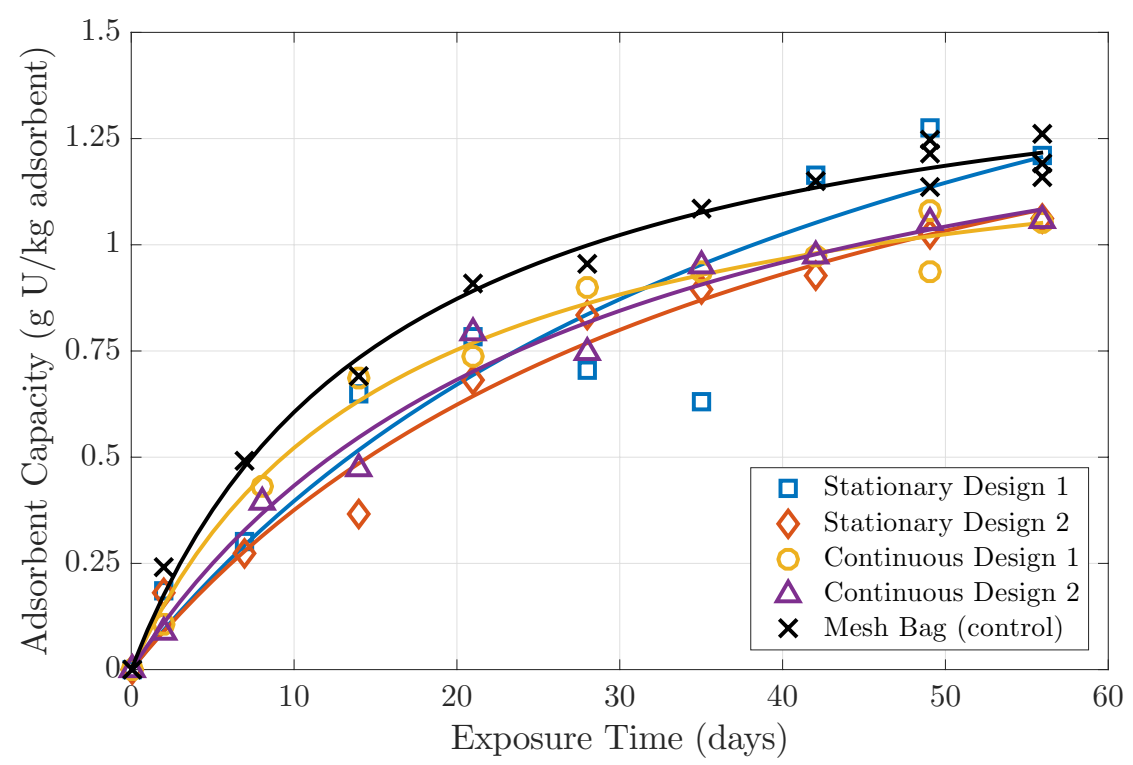

Figure 6-22: Time-dependent measurements of uranium adsorption capacity (g U/kg adsorbent) for the AI8 adsorbent braids enclosed by the different shell designs on the two different systems, and enclosed only by a mesh bag (control). The uranium adsorption capacity was normalized to a salinity of $35 \mathrm{psu}$. Curves drawn through the data represent fitting to a one-site ligand model by (2.2) .
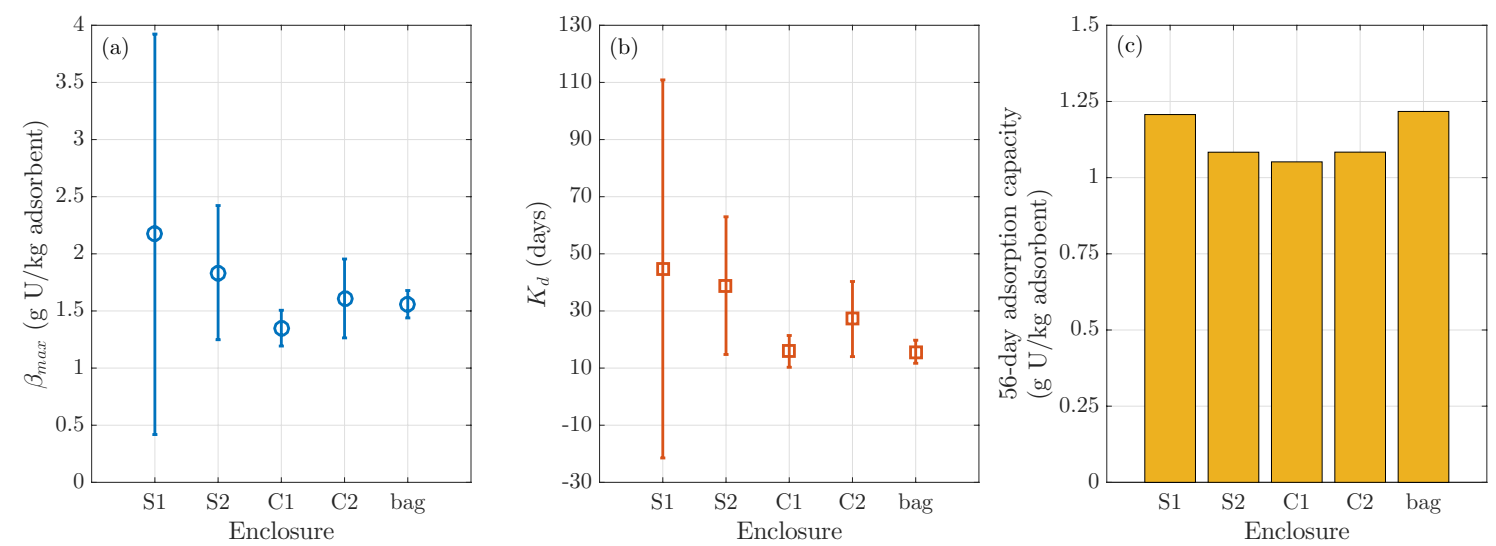

Figure 6-23: The uranium (a) saturation capacity, $\beta_{\max }$, (b) half saturation time, $K_{D}$, and (c) uranium uptake predicted from the one-site ligand model for the adsorbent enclosed different shell designs on the two different systems, and enclosed only by a mesh bag (control).

the shells, may not have helped increase the adsorbent uptake of uranium. This may have been due to the fact that the continuous system was not moving until the last 21 days of the deployment, when the water temperature was colder and towards the end of the deployment, both factors that reduce the uranium uptake of the adsorbent.

This result is further seen in figure 6-24 which shows the total uranium adsorbed ( $\mathrm{g}$-U/kg-adsorbent) at day 56 for the adsorbent fibers in each of the different enclo- 


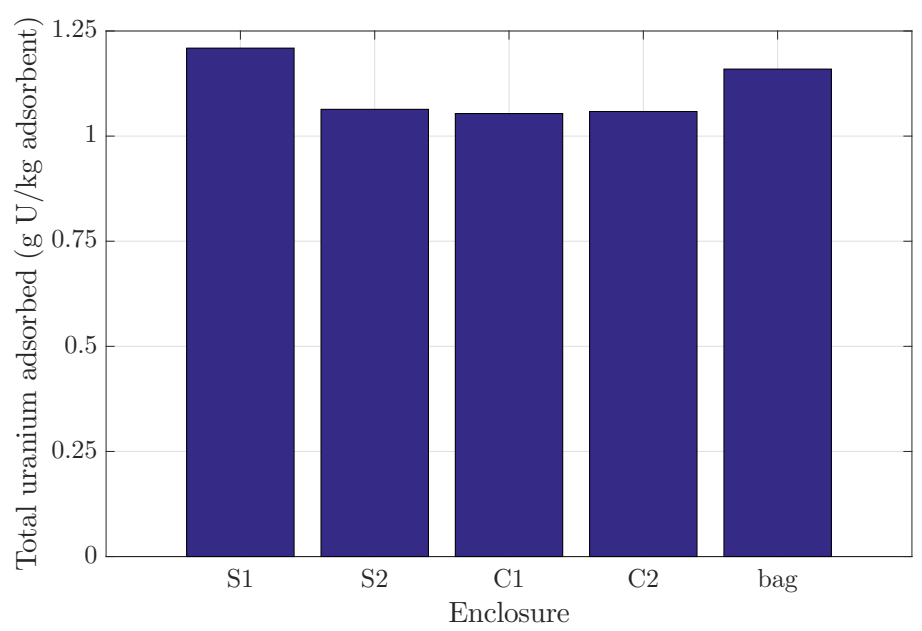

Figure 6-24: The total uranium adsorbed at day 56 by the adsorbent fibers in each enclosure.

sures and systems. The highest uranium uptake was seen by the slotted hole design on the stationary system, which saw a uranium uptake of $1.21 \mathrm{~g}$-U/kg-adsorbent, whereas the same design on the continuous system saw the least amount with $1.06 \mathrm{~g}$ $\mathrm{U} / \mathrm{kg}$-adsorbent, and the control with the adsorbent in the mesh bag on the stationary system saw an uptake of $1.16 \mathrm{~g}$ - $\mathrm{U} / \mathrm{kg}$-adsorbent. The difference in uranium adsorbed by the slotted design on the stationary and continuous systems was only $12.9 \%$, and the difference between the uranium adsorbed by any enclosure and system and the control mesh bag was at most $10 \%$. This suggests again, that there was no significant difference in the uranium adsorbed by fibers in the shells on the different systems as compared to the control, and no significant difference between the uranium adsorbed between the different shell enclosures and systems.

\subsection{Discussion}

This chapter discussed the design, fabrication, and deployment of two prototypes for the harvesting of uranium from seawater using fiber adsorbents. These prototypes were successfully tested in the ocean off the coast of Massachusetts in collaboration with Massachusetts Maritime Academy for a total of 56-days. Throughout the deployment, adsorbent fibers were sampled from the two different prototypes, and from two different shell designs on each prototype, as well as a nylon mesh bag as a control. Analysis of the uranium adsorbing fibers was conducted by the Pacific Northwest National Laboratory and the radium adsorbent fibers by the Woods Hole Oceanographic Institution.

The results showed that, compared to the stationary system, the continuous system shells saw less biofouling. There may have been a few factors which limited the amount of initial biofilm the formed on the shells of the continuously moving system and hence inhibited larger growth.

The first factor is thought to be that the shells in the continuously moving system 
were periodically exposed to air. Every 85 seconds, for a period of 20 seconds, the net was exposed to air as it moved over the upper roller. In comparison, the stationary net system was only exposed to air when adsorbent samples were taken, a total of nine times during the 56-day deployment for no more than 10 minutes at a time. This air exposure of the continuous system could have been enough to deter large amounts of growth from microorganisms and hence limited the amount of macrofouling that could have occurred.

Another cause for the marked reduction in biofouling of the continuous system could have been due to the rubbing of the shells against the PVC pipe on the bottom support or the separation guides. Given that the net was inherently buoyant, once it reached the stationary PVC pipe at the bottom of the system, the shells would rub up against the bottom of the pipe as they moved past. Additional rubbing was noted to occur on various points of the separation guides along the length of the bottom support. This rubbing could have inhibited biofouling and even removed any growth that had already accumulated on the shells.

If either of these factors caused a drastic reduction in biofouling, it lends credence to a few design ideas for mitigating biofouling in such a uranium harvester. Specifically, air exposure could be optimized to reduce the likelihood of microorganism growth, while maximizing the amount of water flow seen by the adsorbent. Secondly, a bristle brush could be added at various parts of the structure to gently brush the shells as they pass, further reducing chances of growth. Additionally, UV light has been shown to have strong antibacterial properties (Lakretz et al., 2010). Adding UV LEDs to a point in the adsorbent net's path could also prevent the formation of biofilm and hence reduce biofouling.

Research has also shown that there exists critical values of current speeds for different species of marine organisms above which fouling biomass is greatly reduced and in general fouling is not possible at speeds greater than $1.5 \mathrm{~m} / \mathrm{s}$ (Railkin, 20103). This suggests that movement of the shell enclosures and adsorbent fibers could also inhibit biofouling growth. However, $1.5 \mathrm{~m} / \mathrm{s}$ is extremely fast and would likely damage the adsorbent. Hence, more research should be done to determine at which speeds the adsorbent is damaged. Furthermore, research should be done to determine at which speeds biofouling is reduced as a function of immersion time. This will give valuable insight into the design of the SMORE system. For instance, if it is found that speeds of $0.5 \mathrm{~cm} / \mathrm{s}$ inhibit biofouling for up to 14 days, this can be used to determine the speed of movement of the adsorbent net as well as the frequency of other biofouling mitigation such as moving the net through a series of UV LEDs. Another thought is that the elution bath may also kill all organisms and hence reduce biofouling. Thus, these results could also be used to determine the frequency of elution of the adsorbent.

Although the adsorbent shells of the continuous system such much less biofouling than those on the stationary system, the adsorbent fibers on the shells in both systems saw no significant difference in weight gain. This discrepancy may have been due to the fact that the continuous system was only up for $37 \%$ of the deployment, with the majority of its uptime occurring at the end of the 56-day deployment. Considering that biofouling occurs within days to weeks of the adsorbent being placed in the ocean, it is likely that both systems saw about the same colonization of organisms 
given that the continuous system was not moving in the first few weeks of the ocean trial. Additionally, when the system was moving, the colder water temperature likely inhibited biofouling for all fibers, regardless of their enclosure or system.

The ocean trial described in this chapter also employed a novel method using the radium adsorbed by $\mathrm{MnO}_{2}$ impregnated acrylic fibers was used to quantify the water flow seen within all the enclosures. The results showed no significant difference in the water flow both shell designs on the same system, however the continuous system shells saw more water flow than the stationary system shells and control bags. This suggests that movement does in fact increase the water flow seen by the adsorbent and further confirms a result of the flume experiment detailed in chapter $⿴$ t that the design of the shell enclosure makes little difference on uptake of the adsorbent inside.

Moreover, the results of the uranium adsorbed by the fibers in each of the shell enclosures and systems showed no significant difference in the uptake of the fibers. Although the shells on the continuous system saw the most water flow, this did not translate into increased uranium uptake. Again, as with the biofouling of the fibers, this may have been due to the fact that the continuous system was not moving reliably until the last few weeks of the test, when the water temperature was colder and towards the end of the deployment, both factors that reduce the adsorbent's uptake. Additionally, results from Ladshaw et all (2017) suggest that for flow rates of $>5.52 \mathrm{~cm} / \mathrm{s}$ the uptake of the adsorbent will no longer increase with increasing velocity. If this is the case, given that the ocean currents are usually $>5.52 \mathrm{~cm} / \mathrm{s}$, the increased water flow to the continuously moving shells will likely not translate to an increase in uranium uptake by the adsorbent fiber. 


\section{Chapter 7}

\section{SMORE Hydrodynamic Response}

This chapter details the hydrodynamic response of a full-scale Symbiotic Machine for Ocean uRanium Extraction. Given that the device is hypothesized to be coupled to a floating offshore wind turbine, it is extremely important that the addition of the uranium harvester device does not adversely affect the hydrodynamic response of the offshore wind turbine. This work was done in collaboration with Jocelyn Kluger (Kluger, 2017).

\subsection{Theory}

As mentioned in chapter $\mathbf{5}$, because offshore wind turbine data is proprietary and often very difficult to use for public work, the present design for SMORE utilizes the StatOil OC3-Hywind spar with a 5-MW NREL turbine, a device well documented by NREL (Jonkman et al., 20().9; Jonkman, 2010).

The problem set up is shown in figure $7-1$. The hydrodynamics of the floating spar buoy are considered in head-on incident waves of amplitude $A$ and frequency $\omega$. These result in coupled degrees of freedom in heave, $X_{3}$, surge, $X_{1}$, and pitch, $X_{5}$, taken about the still water line (SWL).

The linear equations of motion of the system are given by

$$
(\mathbf{M}+\mathbf{A}) \ddot{\xi}+\mathbf{B} \dot{\xi}+\mathbf{C} \xi=\mathbf{X}(t)
$$

where $\mathbf{M}$ is the mass matrix, $\mathbf{A}$ is the added mass coefficient matrix, $\mathbf{B}$ is the linear damping coefficient matrix, $\mathbf{C}$ is the restoring coefficient matrix, $\xi$ is the turbine displacement, and $\mathbf{X}$ is a matrix of the hydrodynamic excitation forces and moments.

Utilizing a linear frequency-domain analysis, the exciting forces and moments due to plane progressive waves will be assumed to be of the form

$$
X_{j}(t)=\Re\left\{\mathbb{X}_{j}(\omega) e^{i \omega t}\right\}, \quad \text { for } j=1, \ldots, 6
$$

where $\mathbb{X}_{j}(\omega)$ is a complex quantity. By virtue of linearity, the turbine's response to wave excitation will be of the form 


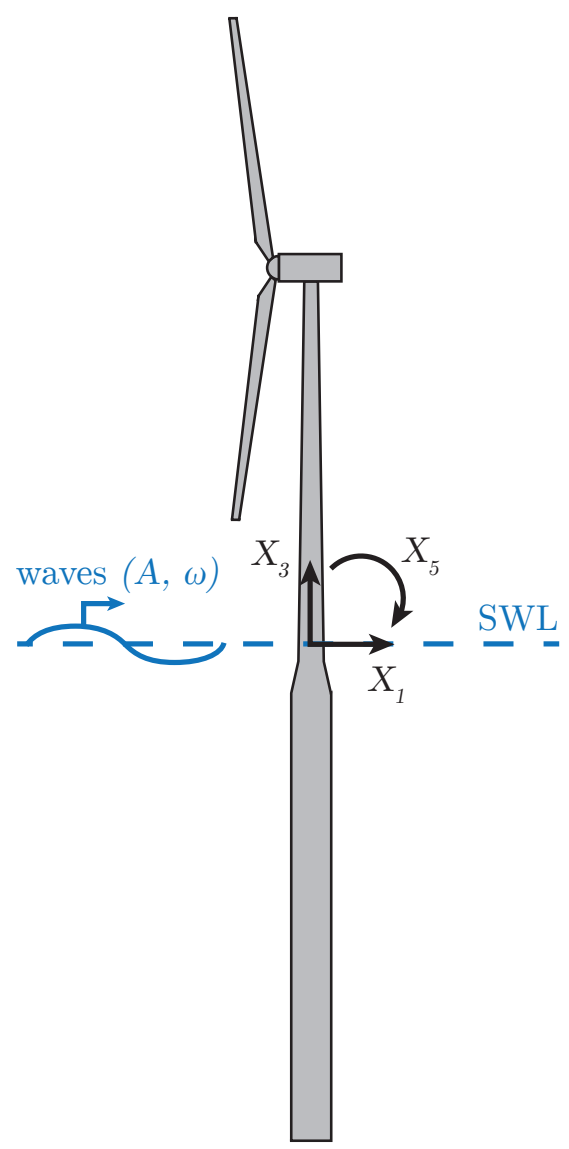

Figure 7-1: Floating spar wind turbine, similar to that of the NREL 5-MW wind turbine mounted on the OC3-Hywind spar (Jonkman et al., 20(1)9; Jonkman, 2010) with incident waves of amplitude $A$ and frequency $\omega$. The motions of the turbine are described about the still water line (SWL).

$$
\xi_{j}(t)=\Re\left\{\Xi_{j}(\omega) e^{i \omega t}\right\}, \quad \text { for } j=1, \ldots, 6,
$$

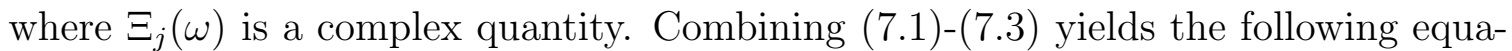
tions of motion in the frequency domain

$$
\left[-\omega^{2}\left(M_{i j}+A_{i j}(\omega)\right)+i \omega B_{i j}(\omega)+C_{i j}\right] \Xi_{j}(\omega)=\mathbb{X}_{i}(\omega) .
$$

The principal seakeeping quantity from a linear seakeeping analysis of a floating body at zero speed is the Response Amplitude Operator (RAO), defined as

$$
R A O_{j}(\omega)=\left|\frac{\Xi_{j}(\omega)}{A / R^{n}}\right|,
$$

where $R$ is the turbine radius, $n=0$ for $j=1,3,5$ and $n=1$ for $j=2,4,6$.

For the case of an arbitrary shape such as an offshore wind turbine, it is common practice to examine the dynamic response numerically. Jonkman (2010) conducted a numerical study of the hydrodynamic response of the OC3-Hywind spar buoy using 
the WAMIT computer program (Newman and Sclavounos, 1988; Lee and Newman, 20166). This program uses a three-dimensional numerical-panel method in the frequency domain to solve response of the turbine to linear wave forcing due to potential flow. The excitation force and RAOs determined by Jonkman (2010) will be used as comparison for the results of the excitation forces and RAOs found in the experiment detailed in this chapter.

\subsection{Experimental Setup}

This experiment was done in collaboration with Jocelyn Kluger, which did the vast majority of the experimental set-up (Kluger, 2017). This section details the scaling of the designs for testing, the experimental facilities used, and the experimental methods used to determine the excitation forces and response amplitude operators.

\subsubsection{Model scaling}

A 1:150 Froude scaled model of two different versions of SMORE and the reference floating wind turbine were developed for testing to determine the wave excitation forces on the structure as well as each design's response. The Froude number is a dimensionless number that describes a ratio of the flow inertia to the external field and is given by

$$
F r=\frac{U}{\sqrt{g L}},
$$

where $U$ is the characteristic velocity, $g$ is acceleration due to gravity, and $L$ is a corresponding characteristic length. The geometric scale, $\lambda$ is defined to be

$$
\lambda=\frac{L_{f}}{L_{m}}
$$

where $L_{f}$ is the full-scale characteristic length and $L_{m}$ is the model scale characteristic length. For this experiment, $\lambda=150$. Matching the model and full-scale Froude numbers amounts to

$$
\begin{aligned}
F r_{f} & =F r_{m} \\
\frac{U_{f}}{\sqrt{g L_{f}}} & =\frac{U_{m}}{\sqrt{g L_{m}}} \\
\Rightarrow U_{m} & =U_{f} \sqrt{\frac{L_{m}}{L_{f}}}=U_{f} \sqrt{\frac{1}{\lambda}} .
\end{aligned}
$$

Hence, the full-scale velocity is scaled by $\sqrt{\lambda}$ to achieve the model scale velocity. This process can be repeated to determine the scale ratio between the model scale and fullscale of various important physical parameters, such as mass, acceleration, and force. 
Table 7.7 details the scale ratios for many of the physical parameters utilized in this experiment. For instance, while acceleration varies only by a ratio of 1 between the model and full scale, the force scales by a factor of $\lambda^{3}$ (Chakrabarti, 1994).

Table 7.1: Scaling ratios for various physical parameters

\begin{tabular}{|llll|}
\hline Variable & Dimensions & Units & Scale Ratio \\
\hline Length & $\mathrm{L}$ & $\mathrm{m}$ & $\lambda$ \\
Mass & $\mathrm{M}$ & $\mathrm{kg}$ & $\lambda^{3}$ \\
Angle & none & $\mathrm{rad}$ & 1 \\
Acceleration & $\mathrm{L} / \mathrm{T}^{2}$ & $\mathrm{~m} / \mathrm{s}^{2}$ & 1 \\
Angular Acceleration & $1 / \mathrm{T}^{2}$ & $1 / \mathrm{s}^{2}$ & $\lambda^{-1}$ \\
Angular Velocity & $1 / \mathrm{T}$ & $1 / \mathrm{s}$ & $\lambda^{-0.5}$ \\
Force & $(\mathrm{M} \times \mathrm{L}) / \mathrm{T}^{2}$ & $\mathrm{~kg} \times \mathrm{m} / \mathrm{s}^{2}$ & $\lambda^{3}$ \\
Wave Height & $\mathrm{L}$ & $\mathrm{m}$ & $\lambda$ \\
Wave Period & $\mathrm{T}$ & $\mathrm{m}$ & $\sqrt{\lambda}$ \\
Velocity & $\mathrm{L} / \mathrm{T}$ & $\mathrm{m} / \mathrm{s}$ & $\sqrt{\lambda}$ \\
Moment of Inertia & $\mathrm{M} \times \mathrm{L}^{2}$ & $\mathrm{~kg} \times \mathrm{m}^{2}$ & $\lambda^{5}$ \\
\hline
\end{tabular}

\subsubsection{Models for testing}

As mentioned, two designs of SMORE were developed for testing, in addition to a scale model of the reference floating wind turbine. The reference floating wind turbine was scaled from dimensions detailed by tonkman et al. (2010.9) and lonkman (2010). Some of the key parameters and their full-scale and model values are detailed in table 7.2, as determined from Jonkman (2010) and Myhr et al. (2014).

The model scale reference floating wind turbine was fabricated using aluminum cylinders turned down to the diameters of the upper and lower turbine spar, an aluminum tube for the turbine tower, a circular plate to simulate the rotor damping on the turbine, and a transition region 3D printed from acrylonitrile butadiene styrene.

Table 7.2: Scaling factors employed for wave model testing

\begin{tabular}{|llll|}
\hline Variable & Unit & Full-Scale & Model Scale \\
\hline Total draft & $\mathrm{m}$ & 120 & 0.8 \\
Tower height & $\mathrm{m}$ & 77.6 & 0.52 \\
Depth to Top of Taper Below SWL & $\mathrm{m}$ & 4 & 0.027 \\
Depth to Bottom of Taper Below SWL & $\mathrm{m}$ & 12 & 0.080 \\
Diameter Above Taper & $\mathrm{m}$ & 6.5 & 0.043 \\
Diameter Below Taper & $\mathrm{m}$ & 9.4 & 0.063 \\
Platform Mass & $\mathrm{kg}$ & 1700000 & 0.5 \\
Tower Mass & $\mathrm{kg}$ & 249718 & 0.074 \\
Ballast & $\mathrm{kg}$ & 5766000 & 1.71 \\
CM Location Below SWL & $\mathrm{m}$ & 89.92 & 0.60 \\
\hline
\end{tabular}


(a)

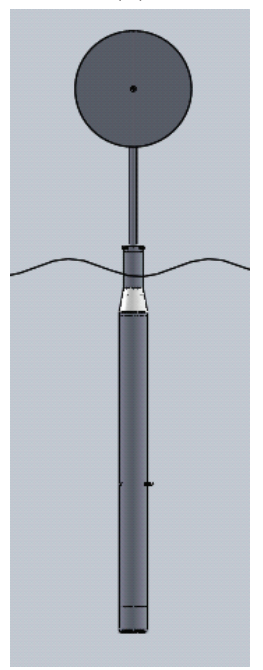

(b)

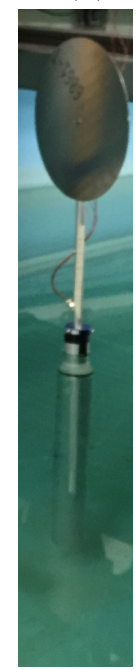

(c)

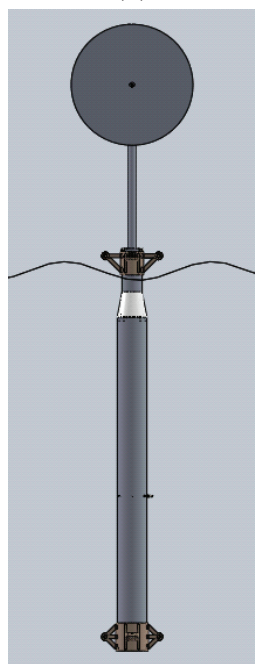

(d)

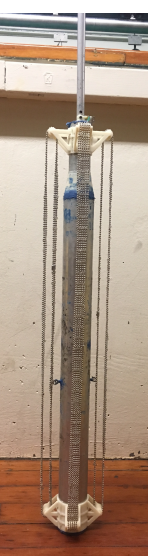

(e)

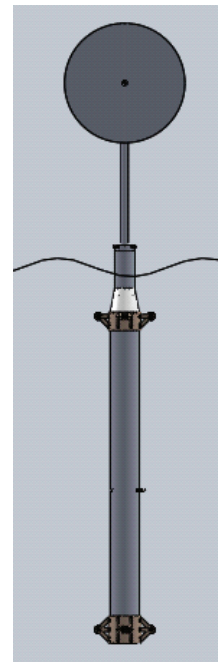

(f)

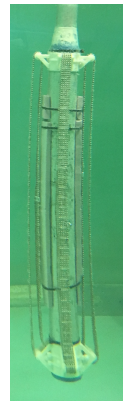

Figure 7-2: Three-dimensional solid models and fabricated designs for the reference floating wind turbine, (a) and (b), the SMORE design with the upper platform out of the water, (c) and (d), and the SMORE design with the upper platform submerged, (e) and (f).

All holes and joints were made waterproof using Permatex ${ }^{\circledR}$ Sensor-Safe Blue RTV Silicone Gasket Maker.

It was thought that a SMORE design such as that described in chapter 1 in which the upper platform with the top rollers were out of the water could have significant risks due to the wave loads near the surface. Given that the wave energy decays exponentially with depth, placing the upper platform underneath the water surface could be one way to mitigate these risks. Therefore, in addition to the reference floating wind turbine, two SMORE designs were tested. In the first, the top set of rollers was placed out of the water at $0.03 \mathrm{~m}$ above the SWL (corresponding to $4.5 \mathrm{~m}$ in a full-scale design). In the second design, the top set of rollers was submerged 0.12 $\mathrm{m}$ below the SWL (corresponding to $18 \mathrm{~m}$ in a full-scale design). Figure [-2] shows the three-dimensional models and fabricated designs used for testing.

For the SMORE designs, The adsorbent ball-chain net was modeled using $3 \mathrm{~mm}$ diameter (\#6 trade size) nickel-plated steel bead chain and the upper and bottom platforms of rollers were 3D printed out of acrylonitrile butadiene styrene. At every five beads, the chains were hot-glued together to mimic the increased rigidity that would be provided by rope cross-members of the ball-chain net. Because $3 \mathrm{~mm}$ diameter bead-chain was not available in plastic, the bead-chain net added extra weight to the model than would be seen in the full-scale version. Therefore, strips of foam were added along the length of the turbine to increase its buoyancy and ensure it had a draft of approximately $0.8 \mathrm{~m}$. By adding the foam strips along the length of the turbine, they mimicked the additional buoyancy that plastic shells with polymer adsorbents would provide to the overall structure. Additionally, the strips were added to the turbine in such a way as to limit the effects of the changed geometry on the turbine's hydrodynamic coefficients. 


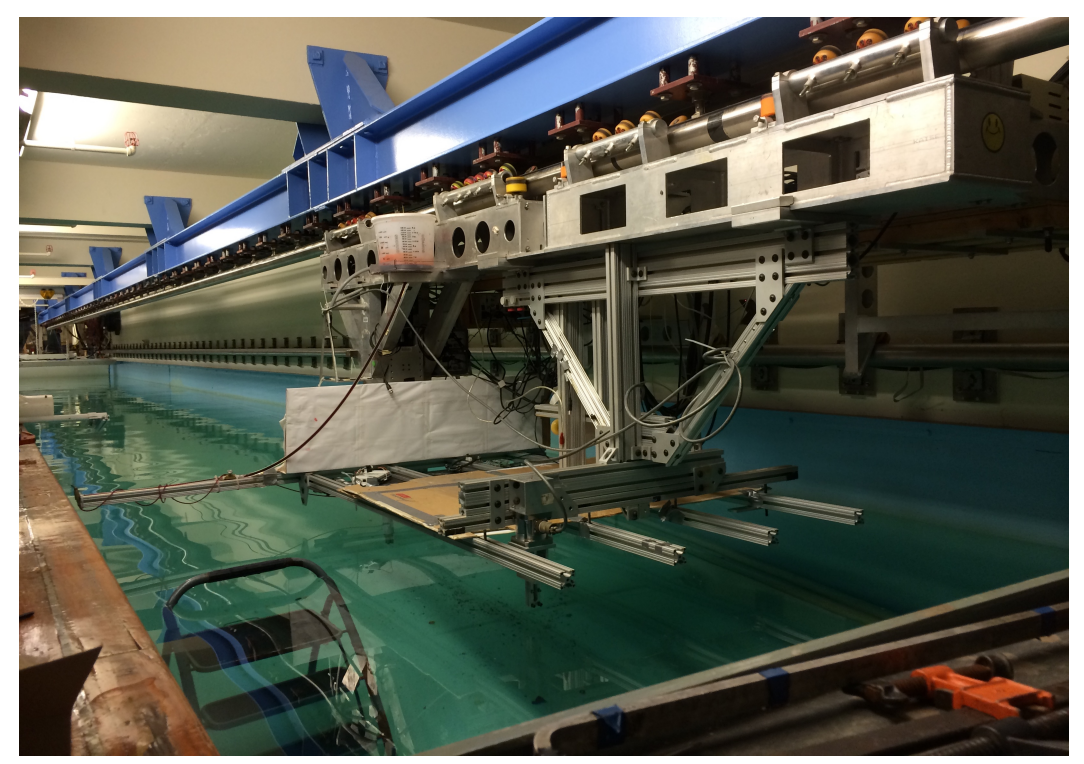

Figure 7-3: MIT Tow Tank where scale model hydrodynamic response tests were conducted of two designs of SMORE and the reference floating wind turbine.

\subsubsection{Experimental facilities}

The tests described in this chapter were conducted in the MIT Tow Tank, shown in figure [-3], which is $30.5 \mathrm{~m}$ long, $2.1 \mathrm{~m}$ wide, and $1.2 \mathrm{~m}$ deep (100 ft long, $8 \mathrm{ft}$ wide, and $4 \mathrm{ft}$ deep) with a wave maker. The wave maker is a hydraulically driven vertical paddle with controllable amplitude and frequency that are programmed using LabView.

As shown in figure 1-4, two wave probes were used to measure the amplitude of the passing waves. One probe was located approximately at mid-width in the tank 11.9 $\mathrm{m}(39.17 \mathrm{ft})$ downstream of the wave maker and another was located approximately $9.5 \mathrm{~m}(31 \mathrm{ft})$ downstream of the wave maker, closer to one of the walls of the tank. The models for testing were located about half-way down the length of the tank.

\subsubsection{Excitation Forces}

In order to measure the excitation forces on the designs, the models were constrained by a set of three load cells in the configuration shown in figure [-5. All load cells were SMT Overload Protected S-Type Load Cells. The heave load cell was rated to $5 \mathrm{lbf}(\sim 22.24 \mathrm{~N})$ and the top and bottom surge load cells were rated to $2.2 \mathrm{lbf}$ $(\sim 9.79 \mathrm{~N})$. Stinger rods measuring $24 \mathrm{~mm}$ and $12 \mathrm{~mm}$ connected the bottom and top surge, and heave load cell to the turbine tower, respectively. Each load cell was connected to a LabView data acquisition unit through a DC powered FUTEK amplifier module to increase the signal readings. The load cells were powered with a stacked dual power supply outputting $\sim 20.7 \mathrm{~V}$ for all trials. The bottom surge load cell was approximately $0.057 \mathrm{~m}$ above the SWL and the top surge load cell was 0.24 $\mathrm{m}$ above the SWL. The surge force was taken to be the sum of the readings of the 


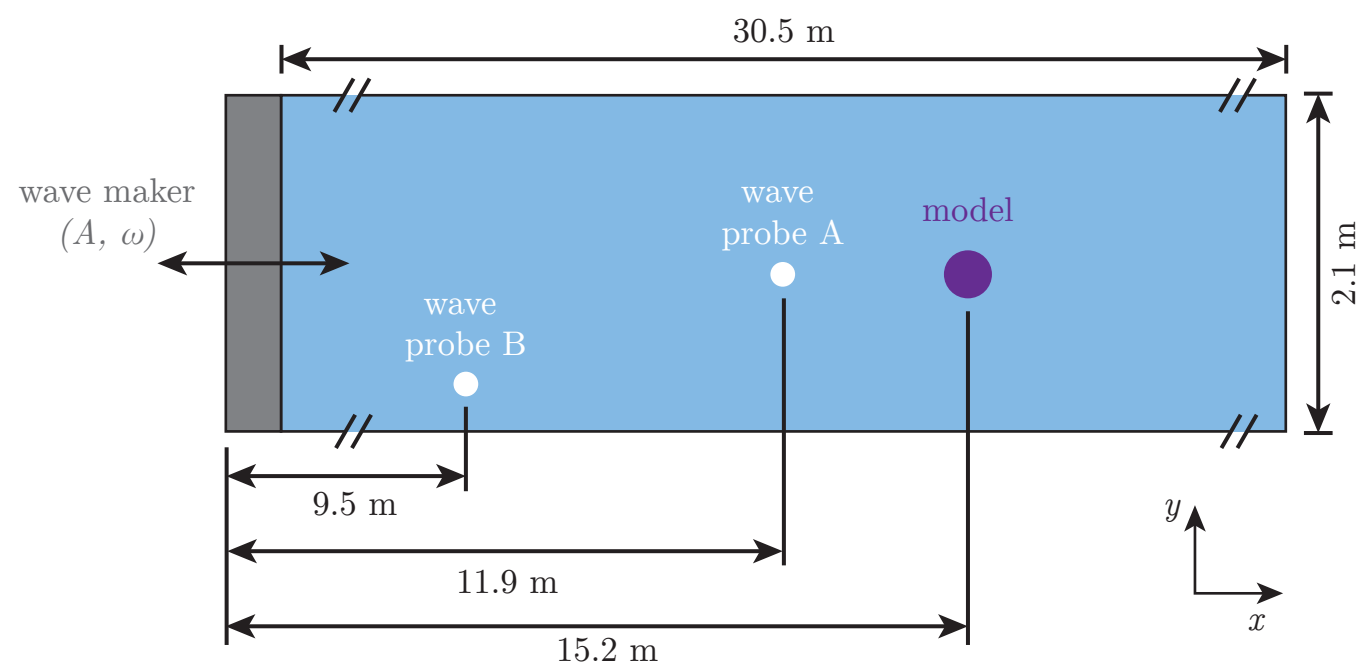

Figure 7-4: Schematic of the experimental configuration. The wave maker on the left was excited at an amplitude, $A$, and frequency $\omega$. Two wave probes (white dots) downstream measured the amplitude incoming to the model (purple) for all tests.

top and bottom load cells. The resulting pitch torque was determined by

$$
X_{5}=X_{1, \text { top }} z_{\text {top }}+X_{1, \text { bottom }} z_{\text {bottom }},
$$

where $X_{1, \text { top }}$ and $X_{1, \text { bottom }}$ are the top and bottom surge force load cell readings, respectively, and $z_{\text {top }}$ and $z_{\text {bottom }}$ are the distances of the load cells to the SWL, respectively.

In order to obtain frequency dependent data for both the wave excitation forces and response amplitude operators, the wave maker was excited at various frequencies and amplitudes for each model test. The wavemaker parameters used for the excitation force measurement experiment are detailed in table $[.3$ where the amplitude was measured by the wave probe. The wavemaker was programed to excite waves for 20 periods for all tests. Because the wave excitation force varies with frequency, $\omega$, the load cell data were filtered using a Fast-Fourier Transform to obtain the amplitude of the forces at the frequency of interest.

\subsubsection{Response Amplitude Operators}

To determine the response amplitude operators of the models, tests were conducted in which the models were freely floating and an accelerometer mounted to the tower of the turbine measured heave, surge, and pitch motions. The accelerometer used in this setup was a SparkFun 9 Degree of Freedom Sensor Stick, utilizing the LSM9DS1 motion-sensing system-in-a-chip which required an operating voltage of $3.3 \mathrm{~V}$. The accelerometer was configured to measure $\pm 2 \mathrm{~g}$ in acceleration and $\pm 245 \mathrm{deg} / \mathrm{s}$ in angular velocity using a Teensy 3.2 USB development board powered through a laptop USB port. The experimental setup is shown in figure [7-6.

Because the accelerometer was mounted above the SWL, the measurements had 
Table 7.3: Parameters for Excitation Force Experiment

\begin{tabular}{|l|c|c|c|l|l|l|}
\cline { 2 - 7 } \multicolumn{1}{c|}{} & \multicolumn{9}{c|}{ Amplitude (m) } \\
\cline { 2 - 7 } \multicolumn{1}{c|}{} & FWT Alone & \multicolumn{2}{c|}{ SMORE Above } & \multicolumn{2}{l|}{ SMORE Below } \\
\hline Frequency (Hz) & Small & Large & Small & \multicolumn{1}{c|}{ Large } & Small & Large \\
\hline 0.4 & & 0.0088 & 0.0068 & 0.0138 & 0.007 & 0.014 \\
\hline 0.5 & 0.0023 & 0.0079 & 0.0069 & 0.0088 & 0.0069 & 0.01 \\
\hline 0.6 & 0.0131 & & 0.0139 & 0.03 & 0.0122 & 0.024 \\
\hline 0.7 & 0.0087 & 0.0162 & 0.0076 & 0.015 & 0.0078 & 0.0151 \\
\hline 0.8 & 0.0096 & 0.0175 & 0.008 & 0.0187 & 0.009 & 0.0175 \\
\hline 1.1 & 0.0101 & 0.0211 & 0.0051 & 0.0216 & 0.0109 & 0.0207 \\
\hline 1.6 & 0.0096 & 0.0154 & 0.01 & 0.0161 & 0.0109 & 0.0144 \\
\hline
\end{tabular}

to be translated by

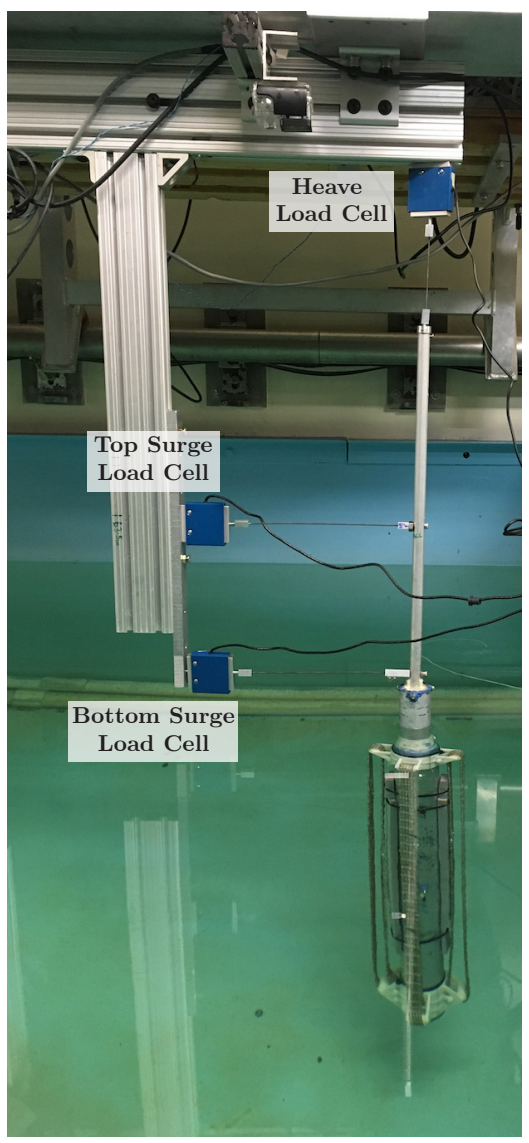

Figure 7-5: Experimental setup of the excitation force test. One load cell was used to measure heave and two were used to measure surge. The difference in measurement of the two surge load cells and their distance from the SWL was used to determine the pitch torque. 


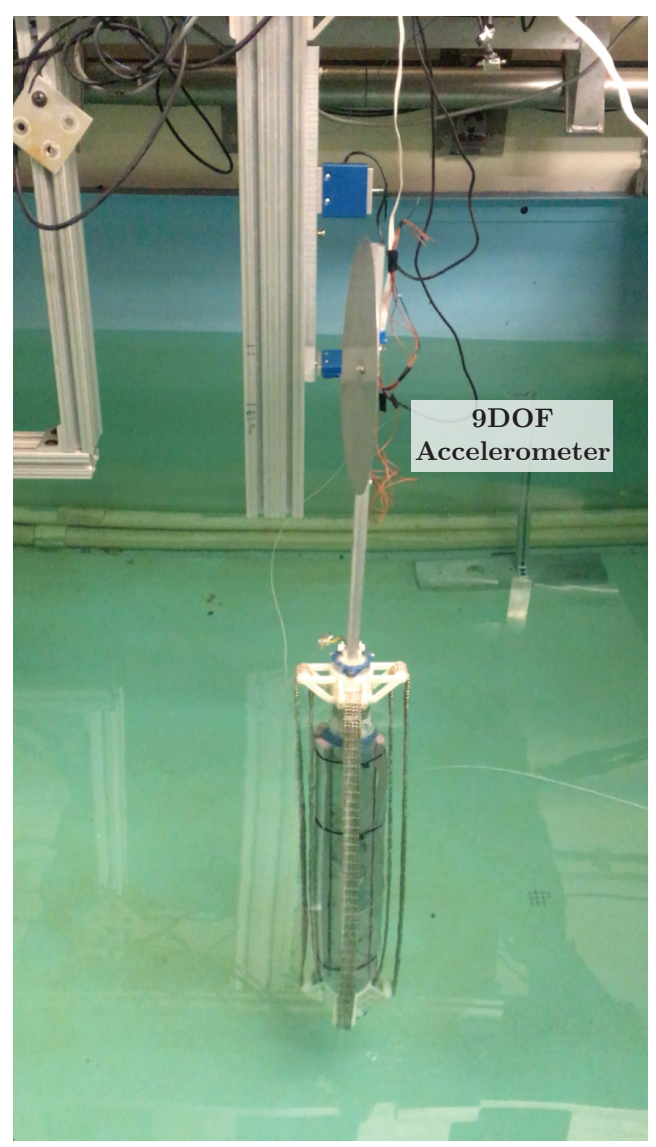

Figure 7-6: Experimental setup of the RAO test. A 9DOF accelerometer mounted to the tower of the turbine was used to measure heave, surge, and pitch motions.

$$
\mathbf{a}^{\mathrm{SWL}}=\mathbf{a}^{0}+\boldsymbol{\omega}^{\mathrm{T}} \times\left(\boldsymbol{\omega}^{\mathrm{T}} \times \mathbf{r}^{0, \mathrm{SWL}}\right)+\boldsymbol{\alpha}^{\mathrm{B}} \times \mathbf{r}^{0, \mathrm{SWL}},
$$

where $\mathbf{a}^{\mathrm{SWL}}$ is the acceleration of the turbine at the SWL, $\mathbf{a}^{0}$ is the acceleration of the turbine measured at the accelerometer, $\boldsymbol{\omega}^{\mathrm{T}}$ is the angular acceleration of the turbine as measured by the accelerometer, $\mathbf{r}^{0, \mathrm{SWL}}$ is the position vector from the location of the accelerometer to the SWL, and $\boldsymbol{\alpha}^{\mathrm{T}}$ is the angular acceleration of the turbine, as determined from the accelerometer. Note that because the turbine is assumed to be a rigid body, the angular velocity, $\boldsymbol{\omega}^{\mathrm{T}}$, and angular acceleration, $\boldsymbol{\alpha}^{\mathrm{T}}$ are the same at all points on the body.

In order to obtain frequency dependent data for the response amplitude operators, the wave maker was excited at various frequencies and amplitudes for each model test. The test parameters used for the RAO determination experiment are detailed in table 7.4 where the amplitude was measured by the wave probe. The wavemaker was programed to excite waves for 20 periods for all tests. Given that the response amplitude operator is a function of frequency, $\omega$, the acceleration and angular velocity data were filtered using a Fast-Fourier Transform to obtain the amplitude of the response at the frequency of interest. 
Table 7.4: Parameters for RAO Experiment

\begin{tabular}{|c|c|c|c|c|c|c|c|}
\hline \multirow{3}{*}{ Frequency (Hz) } & \multicolumn{7}{|c|}{ Amplitude (m) } \\
\hline & \multicolumn{3}{|c|}{ FWT Alone } & \multicolumn{2}{|c|}{ SMORE Above } & \multicolumn{2}{|c|}{ SMORE Below } \\
\hline & Small & Medium & Large & Small & Large & Small & Large \\
\hline 0.4 & 0.004 & & & 0.0069 & 0.0138 & 0.0069 & 0.0135 \\
\hline 0.5 & 0.0046 & & 0.0063 & 0.0072 & 0.009 & 0.0068 & 0.0074 \\
\hline 0.6 & 0.005 & 0.0054 & 0.0163 & 0.0128 & 0.0273 & 0.0147 & 0.0286 \\
\hline 0.7 & 0.0074 & 0.0115 & 0.0121 & 0.0079 & 0.0166 & 0.0079 & 0.0154 \\
\hline 0.8 & 0.0089 & 0.0154 & 0.0229 & 0.0092 & 0.0196 & 0.0092 & 0.0183 \\
\hline 1.1 & 0.0107 & 0.0179 & 0.0272 & 0.0109 & 0.0209 & 0.008 & 0.0184 \\
\hline 1.6 & 0.0113 & 0.0172 & 0.0232 & 0.0103 & 0.016 & 0.0098 & 0.0162 \\
\hline
\end{tabular}

\subsection{Results}

The results for the excitation force and RAOs for all models tested are shown and discussed in this section. All experimentally determined results are compared to the numerical results determined by Wonkman (2010) for the case of the unmodified, reference wind turbine.

\subsubsection{Excitation Forces}

\section{Surge Excitation Force}

Figure [-7 shows the full-scale surge excitation force for the reference turbine, SMORE with the upper platform above the water, and SMORE with the upper platform submerged. In the case of the unmodified wind turbine, the surge excitation force is slightly increased as compared to that determined numerically by donkman (2000).
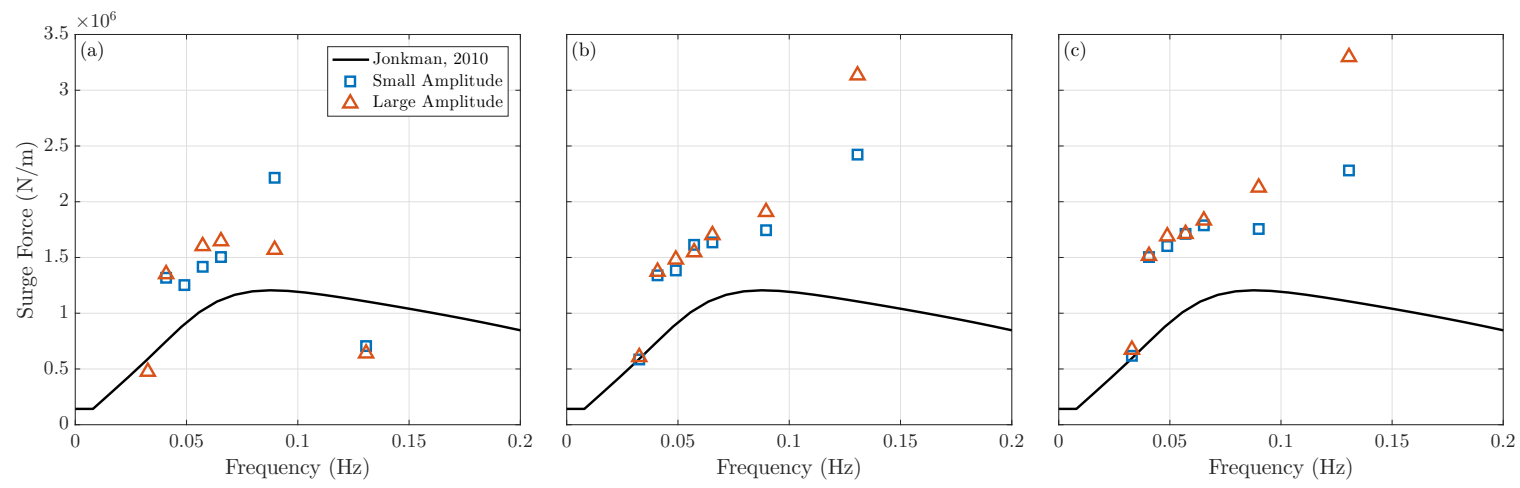

Figure 7-7: Full-scale surge excitation force as determined by the load cell tests for small (blue squares) and large (red triangles) amplitude waves as compared to that numerically determined by $.0 n k m a n$ (2010) (black line) for the (a) reference floating wind turbine, (b) SMORE design with the upper platform above the water, and (c) SMORE design with the upper platform submerged. 
This discrepancy could be due to slight differences in the model turbine's properties as compared to the full scale turbine. For instance, the data from tonkman (2010) mentions the platform mass, including ballast to be 7466 tonnes. Myhr et al. (2014) details the platform uses 1700 tonnes of steel, therefore it was assumed the difference in mass, 5766 tonnes, was ballast in the turbine, which may not actually be the case. Although the magnitude of the surge excitation force is increased for the experimentally tested turbine as compared to those determined numerically, the trends are consistent between the experimentally and numerically determined surge excitation force. Additionally, there is good agreement in the excitation force for waves of different amplitudes excited at the same frequency, as expected.

For the case of both SMORE (figure [-7b) with the upper platform out of the water and with the platform submerged (figure $[-7 \mathrm{c}$ ), the surge excitation force is in good agreement with that experimentally measured in the reference floating wind turbine test (figure 7-7a). This suggests that the SMORE design with the upper platform above the water surface does not have significantly difference excitation forces as those acting on the unmodified wind turbine.

\section{Heave Excitation Force}

As seen in figure $7-8 \mathrm{la}$, there is excellent agreement in the heave excitation force for the unmodified turbine as determined numerically by donkman (2010) and measured by the load cells. The experimentally determined heave excitation force for SMORE with the upper platform above the water (figure [-8]b) agrees well with the heave excitation force for the unmodified turbine both determined experimentally and by Jonkman (2010). On the other hand, the heave excitation force of SMORE with the upper platform submerged (figure [-8c) shows an increase in the full-scale heave excitation force compared to those both determined experimentally for the unmodified turbine (figure 7-8la) and those found by Donkman (2010). This is expected as the
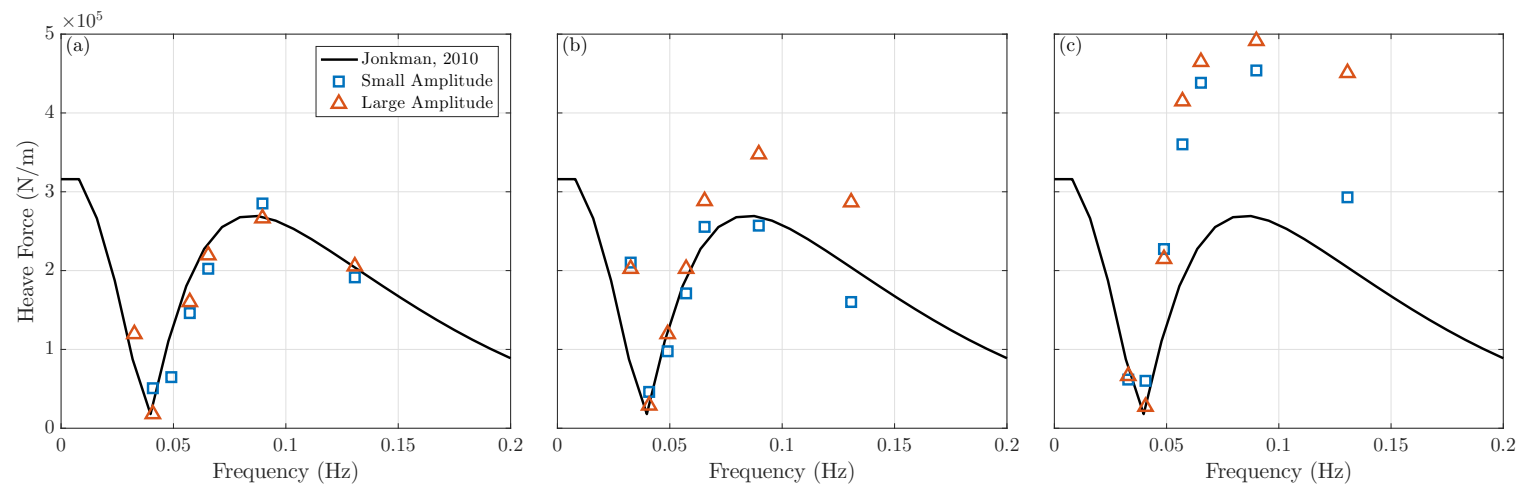

Figure 7-8: Full-scale heave excitation force as determined by the load cell tests for small (blue squares) and large (red triangles) amplitude waves as compared to that numerically determined by Jonkman (2010) (black line) for the (a) reference floating wind turbine, (b) SMORE design with the upper platform above the water, and (c) SMORE design with the upper platform submerged. 
submerging of the upper platform adds a significant amount to the water plane area of the system and hence affects the heave hydrostatic restoring force. Although the heave excitation force is increased, the same trend for the case of this SMORE design as with the unmodified turbine can be seen, suggesting that the dominant excitation frequencies remain the same.

\section{Pitch Excitation Torque}

Similarly to the experimentally determined surge excitation force, the and pitch excitation torque for the unmodified turbine (figure [-.9a) is slightly increased as compared to that determined numerically by lonkman (2010). Because the surge and pitch motions of a floating wind turbine are coupled, this discrepancy is likely due to the same reason the measured surge excitation force is larger than that predicted Jonkman (2010). Although the magnitude of the pitch excitation torque is increased for the experimentally tested turbine as compared to that determined numerically, the trends are consistent between the experimentally and numerically determined pitch excitation torque.
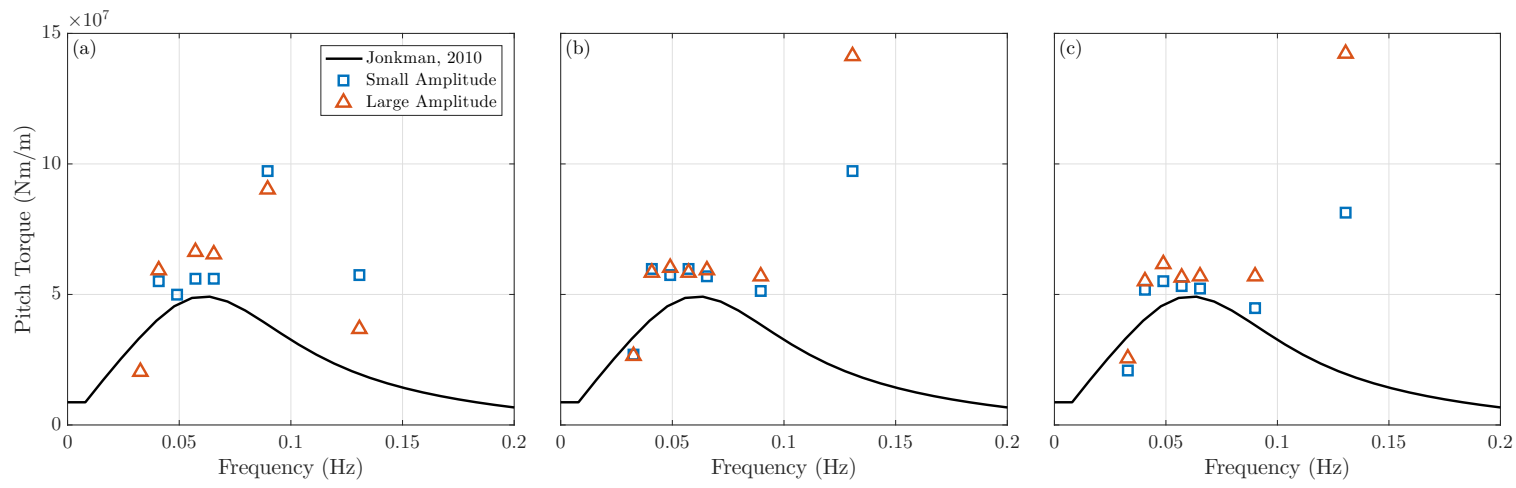

Figure 7-9: Full-scale pitch excitation torque as determined by the load cell tests for small (blue squares) and large (red triangles) amplitude waves as compared to that numerically determined by tonkman (2010) (black line) for the (a) reference floating wind turbine, (b) SMORE design with the upper platform above the water, and (c) SMORE design with the upper platform submerged.

\subsubsection{Response Amplitude Operators}

\section{Surge RAO}

For the unmodified floating wind turbine, the experimentally determined surge RAO (figure $7-10 \mathrm{a}$ ) is slightly increased from that determined numerically by lonkman (2010)). The surge RAO for both SMORE with the upper platform above the water (figure [-10]b) and SMORE with the upper platform submerged (figure $1-10 \mathrm{c}$ ) show good agreement with the experimentally determined surge RAO for the unmodified turbine. This suggests that the surge response of the turbine is unaffected by the addition of either SMORE design. 

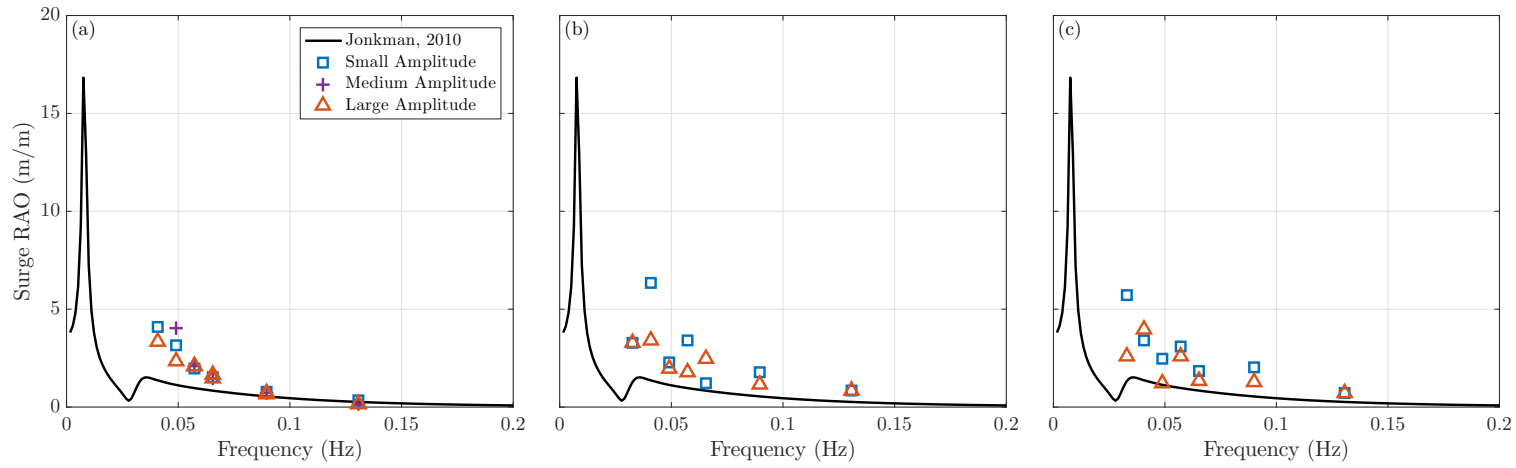

Figure 7-10: Full-scale surge RAO as determined by the accelerometer tests for the (a) reference floating wind turbine, (b) SMORE with the upper platform above the water, and (c) SMORE with the upper platform submerged, for small (blue squares), medium (purple crosses) and large (red triangles) amplitude waves as compared to that numerically determined by donkman (2010) (black line).

\section{Heave RAO}

In the case of the heave RAO, the unmodified floating wind turbine experimentally determined response agrees extremely well with that numerically determined by Ionkman (2010) (figure [-Tla). The SMORE design with the upper platform above the water also exhibits a similar heave response (figure $[-[-1 b$ ), suggesting the addition of this type of SMORE design does not affect the turbine's heave motion. On the other hand, the heave response is slightly decreased for the SMORE design with the upper platform submerged (figure $[-\Pi \mathrm{c}$ ) from that determined by Jonkman (2010). This is likely due to the additional damping in heave provided by all of the submerged structures on the floating wind turbine.
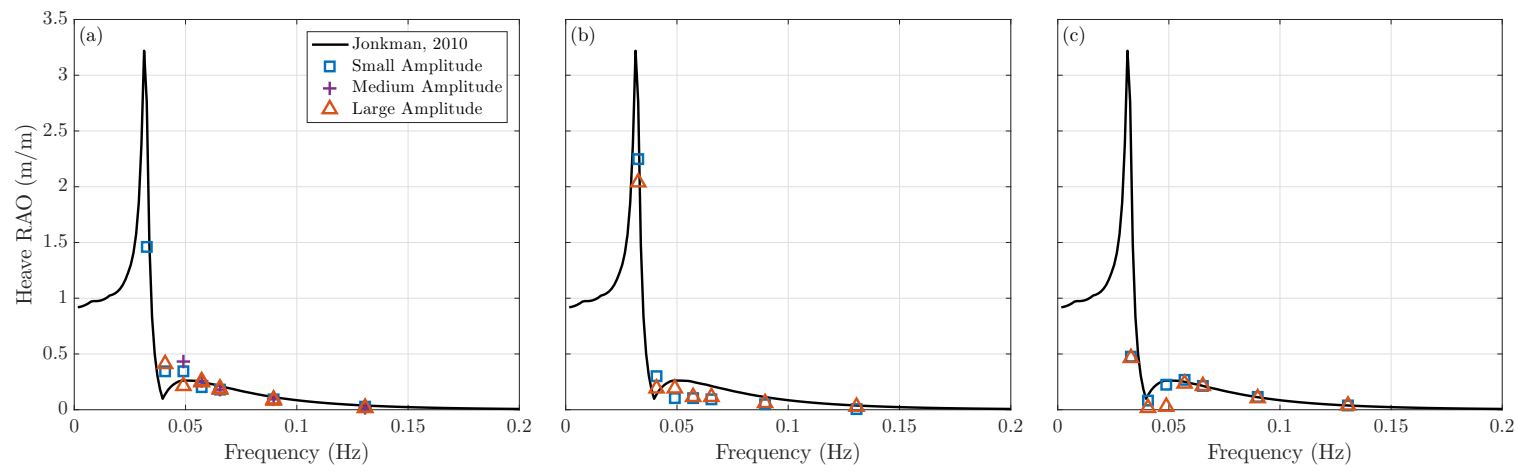

Figure 7-11: Full-scale heave RAO as determined by the accelerometer tests for the (a) reference floating wind turbine, (b) SMORE with the upper platform above the water, and (c) SMORE with the upper platform submerged, for small (blue squares), medium (purple crosses) and large (red triangles) amplitude waves as compared to that numerically determined by Jonkman (2010) (black line). 


\section{Pitch RAO}

As with the heave RAO, the unmodified wind turbine's measured pitch RAO (figure [-12a) agrees well with that determined numerically by Wonkman (2010). The same is true for the pitch RAO for the SMORE design with the upper platform submerged (figure $[-12 \mathrm{c}$ ), suggesting this modification to the turbine does not affect its pitch motions. On the other hand, the upper platform above the water shows a decrease in the pitch response, especially near the pitch resonant frequency for the unmodified turbine (figure $[-12 \mathrm{~b}$ ). This is likely due to the additional roller platform near the bottom of the turbine which acts to increase the restoring pitch torque.
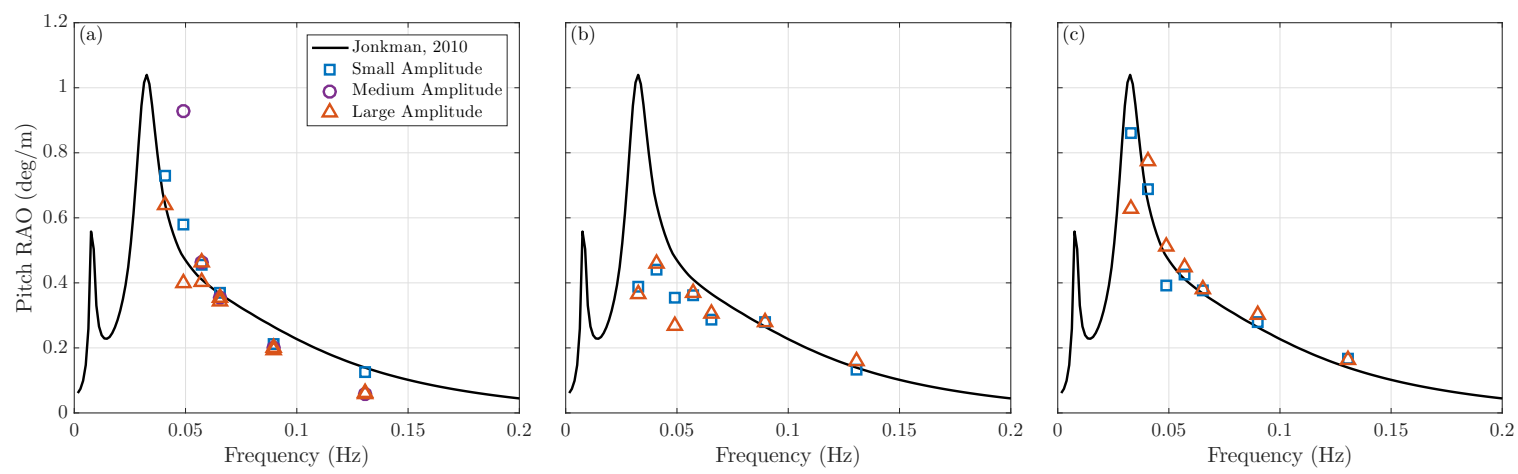

Figure 7-12: Full-scale pitch RAO as determined by the accelerometer tests for the (a) reference floating wind turbine, (b) SMORE with the upper platform above the water, and (c) SMORE with the upper platform submerged, for small (blue squares), medium (purple crosses) and large (red triangles) amplitude waves as compared to that numerically determined by donkman (2010) (black line).

\subsection{Discussion}

The results of the model testing of various SMORE designs indicate that for both designs, one in which the upper platform is out of the water and another in which it is submerged, there is little effect on the overall hydrodynamics of the wind turbine to which the uranium harvester is attached. While the magnitude of the excitation forces or turbine response to these waves may vary from an unmodified wind turbine, in general the variations are minor. Furthermore, the resonant peaks of the turbine response are not affected by the addition of either of the SMORE systems. This is key because an offshore wind turbine is tuned such that its resonant frequencies for various motions will be out of the frequency of storms. The resonant frequencies of the unmodified turbine are in the very low frequency range $(0.0077-0.0313 \mathrm{~Hz}, \sim 0.04-$ $0.3 \mathrm{rad} / \mathrm{s}$ ), for which there is little energy in typical ocean spectra. The addition of either SMORE design does not impact this resonant frequency.

Although both SMORE designs do not affect the dynamics of the turbine they are attached to, other considerations should be taken into account when determining the design of SMORE. Specifically, a SMORE design with the upper platform submerged 
may have added benefits like reduced light and air contact on the adsorbent, which could translate to reduced biofouling. Additionally, submerging the upper platform to below the ocean surface could greatly reduce wave loads on the uranium harvester since wave forcing decreases exponentially with depth. On the other hand, designing a chemical system to extract uranium from the adsorbent for SMORE with a submerged upper platform will likely be more difficult than if the platform were above the water. These considerations must be further investigated before designing a system for a large-scale pilot study. 


\section{Chapter 8}

\section{SMORE Cost-Analysis}

This chapter describes the economic analysis technique used to determine the uranium production cost for previous deployment strategies (Schneider and Sachde, 201:3; Schneider and Linder, 2014; Byers and Schneider, 2015; Byers, 2015; Byers et al., 2016; Haji et al., 2017). It is then used to analyze the uranium production cost from a SMORE device. The resulting cost is compared to that from the reference deployment scheme (which has been used as the base case for cost estimates to date). All costs are presented in 2015 dollars. The technique used in this chapter is described in detail by Byers (2015) and the work presented in this chapter was done in collaboration with Dr. Erich Schneider and Maggie Flicker Byers from the University of Texas at Austin.

\subsection{Discounted Cash Flow Methodology}

The economic cost analysis considers the deployment strategy to consist of three steps. In the first step, the adsorbent is produced using radical polymerization. Next, it is fabricated into either a braid (for the reference deployment scheme) or filament balls (for SMORE and any other strategies in which a shell is used to enclose the adsorbent) and deployed. In the third step (also referred to as the back end), a chemical bath is used to elute the uranium and other metal ions from the polymer. The costs of these three steps are considered individually and then summed and the steps are repeated until it is no longer economically effective to do so.

For each of the three steps, the capital, operating, and decommissioning costs are evaluated, resulting in a timeline of when costs are incurred. A discounted cash flow technique in which the time value of money is taken into account is then used to sum these costs (Park, 2016). In order to compare costs of seawater uranium production for various deployment strategies, the discounted cash flow technique is used to track the lifecycle of a unit mass of adsorbent from its initial fabrication through its reuses and final disposal. The overall procedure relies primarily on the Economic Modeling Working Group (EMWG) Of the Generation IV International Forum cost estimation guidelines as a reference (EMW, 20(1) $)$.

The lifecycle discounted cash flow approach is given by 


$$
A F=\frac{\left(1-\frac{1}{1+i_{c}}\right)^{t_{p r o j}}}{i_{c}}
$$

where $A F$ is the amortization factor, $i_{c}$ is the interest rate on capital, and $t_{\text {proj }}$ is the length of time over which the debt is financed. $A F$ is used to derive a schedule on which capital expenses are made payable. In this technique, the point of initial deployment of the adsorbent is set to $t=0$. This analysis also assumes that all costs are incurred when they are encountered in the lifecycle. Moreover, all unit masses of adsorbent are treated as having identical timelines, one of which is shown in figure $8-1$.

Each cost component is normalized to common units, such as cost per ton of adsorbent produced or cost per ton of adsorbent deployed depending on the process step. The unit cost for each process is then found by summing all cost components for a given system process. For mooring and back end costs, the lifecycle unit cost of these processes is found by discounting the unit costs from the future at which each use occurs back to the present, and summed over all $N$. The only costs components that occur once in the lifecycle are the adsorbent production and disposal, and are therefore discounted to the past or the future, respectively. The total lifecycle cost of a unit of adsorbent, $l u c_{a d s}$ is the sum of the total system steps. The final cost of uranium is then given by dividing $l u c_{a d s}$ by the discounted uranium recovery rate per unit adsorbent, $l_{c}$ (the details of this are described in Byers (2015)):

$$
u c_{u}=\frac{l u c_{a d s}}{l_{c}}
$$

\begin{tabular}{|ll|}
\hline Capital Costs & Production Scaling \\
Operating Costs & Deployment Scaling \\
D\&D Costs & \\
\hline
\end{tabular}

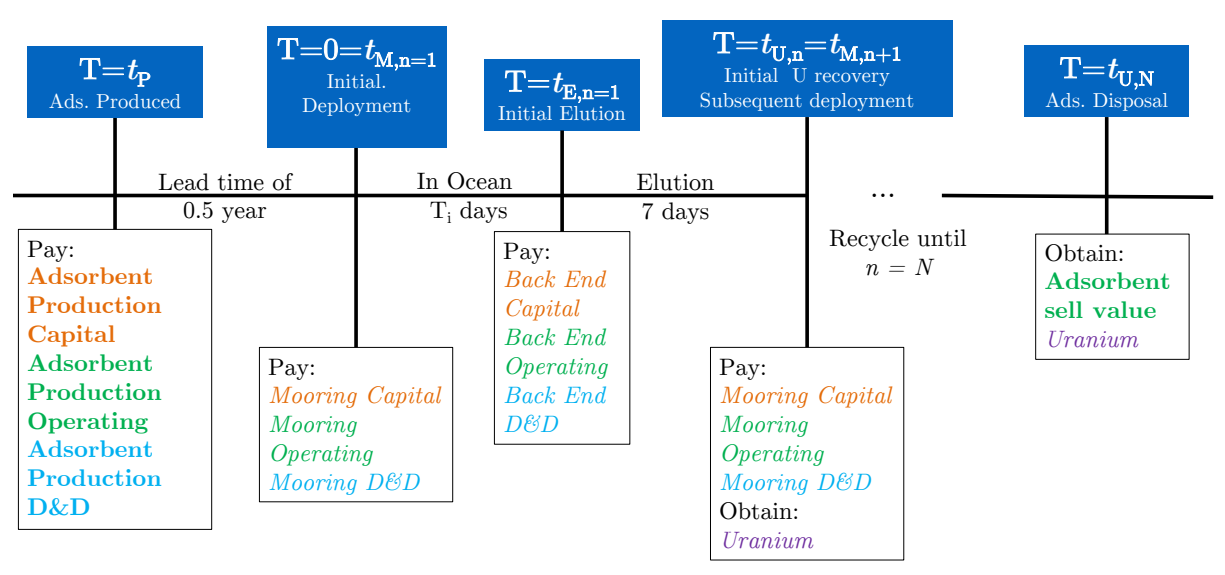

Figure 8-1: Timeline of the lifecycle of an adsorbent following the discounted cash flow technique. 


\subsection{Cost Components}

The components of the production cost of uranium from seawater are broken down into the following categories:

1. Adsorbent Production

2. Mooring and Deployment

3. Elution and Regeneration (also known as Back End)

Relationships and reference data described below can be found in Byers (2015); Schneider and Sachde (2013) and ?.

\subsubsection{Adsorbent Production Cost}

The adsorbent production process involves three steps: fiber spinning, irradiation, and grafting. It can be broken down into capital costs, operating costs, and disposal and decommissioning costs.

\section{Capital Costs}

The main contributors to the capital cost of the adsorbent production are the cost of the buildings and equipment used for manufacturing the adsorbent backbone, grafting the ligand, fabricating the adsorbent into either a braid or a koosh ball, and conditioning the fibers before they are sent out to sea. Some of the costs are derived from scaling the costs of existing and theoretical chemical plants. Miscellaneous factors such as land, contractor's fees, and electrical systems are estimated to be a fraction of the equipment cost. These costs are considered to be amortized over the project lifetime and are a function of the amortization factor, $A F$ from (‥]).

\section{Operating Costs}

In addition to capital cots, there will be costs associated with operating and maintaining the facility and equipment for the adsorbent production process. Specifically, the main cost components for the adsorbent operating costs are the labor, utilities, and material costs. All chemicals are assumed to be used with $100 \%$ efficiency of their nominal values. For the chemicals which are known to be reusable, only $90 \%$ is assumed to be recycled to account for some inevitable loss. Additionally, miscellaneous costs such as taxes, contingency, etc. scale with the production rate of the finished adsorbent. Incineration, considered to be the disposal method for select materials, and hazardous waste disposal are both included in the cost estimate. 


\section{Disposal and Decommissioning (D\&D) Costs}

When the project is complete, all facilities and equipment related to the adsorbent production must be decommissioned and/or disposed of. For this cost analysis, these disposal and decommissioning (D\&D) costs are estimated to be $10 \%$ of the adsorbent production capital cost. The $\mathrm{D} \& \mathrm{D}$ costs are considered to be made payable on a yearly basis and the funds set aside for the $\mathrm{D} \& \mathrm{D}$ of facilities earn interest in a sinking fund throughout the lifetime of the project.

\subsubsection{Mooring and Deployment Cost}

Mooring equipment and marine transportation are required for the mooring, deployment, and retrieval of the adsorbents at the selected site. As with the adsorbent production, the costs associated with this aspect of seawater uranium production can be categorized into capital costs, operating costs, and disposal and decommissioning costs.

\section{Capital Costs}

In the case of the mooring and deployment, the capital cost is determined by the strategy used for the deployment of the adsorbent at sea. Unlike the adsorbent production, because this process occurs at sea there is no building cost. Rather, the major cost components include the cost of boats to install and retrieve the adsorbent and any equipment needed for operations of a specific deployment strategy. For instance, the capital cost components of the reference deployment strategy include mooring chains to keep the buoyant adsorbent braids weighted down to the seafloor. On the other hand, in the case of SMORE, the capital cost includes all additional equipment attached to the wind turbine to secure or cycle the adsorbent shell nets, such as the steel rollers.

\section{Operating Costs}

As with the adsorbent production, the mooring and deployment process includes costs for operation and maintenance of the deployment. As in the adsorbent production process, these chemicals are assumed to be used with $100 \%$ efficiency and $90 \%$ recyclability. Like the mooring and deployment capital costs, the operating costs will vary depending on the deployment strategy. For example, in the case of the reference deployment strategy operating costs include the cost of labor, ships, and fuel for frequent visits for retrieval and redeployment of the adsorbent between the field site and a mothership on which the elution process takes place. Additional cost components contributing to the operating and maintenance cost of the mooring and deployment process include the off-shore lease, which is a function of the area required for the adsorbent field. 


\section{Disposal and Decommissioning (D\&D) Costs}

All facilities and equipment related to the mooring and deployment must be decommissioned and/or disposed of at the end of the project's lifetime. As with the adsorbent production, these $\mathrm{D} \& \mathrm{D}$ costs are estimated to be $10 \%$ of the mooring and deployment capital cost that are made payable on a yearly basis and the funds set aside for the $\mathrm{D} \& \mathrm{D}$ of facilities earn interest in a sinking fund throughout the lifetime of the project.

\subsubsection{Elution and Regeneration Cost}

Finally, the elution and regeneration process also incurs capital, operating, and D\&D costs. This process includes the elution of metals from the adsorbent, the purification of uranium to transform it to purified ammonium diuranate (ADU), and any reconditioning required to prepare the adsorbent for redeployment. The reconditioning of the adsorbent only occurs when the adsorbent will be redeployment and therefore is not considered to occur after the last adsorbent reuse. These costs are referred to as "back end" costs.

\section{Capital Costs}

The capital costs related to the back end include the costs of buildings and equipment needed to both elute the uranium off the braided adsorbent, to purify the products to ADU, and recondition the polymer for reuse. These costs include the expenses for building the facilities, stocking them with necessary equipment and initial inventory, and other expenses. One month's worth of initial chemical inventory is included in the capital cost. These costs will vary between deployment strategies as the reference deployment strategy considers the elution and regeneration steps to take place on a mothership close to the deployment site, while the SMORE strategy considers the elution and regeneration steps to take place at the deployment site. However, the purification step to ADU is still assumed to be preformed at a dedicated onshore facility for all deployment strategies.

\section{Operating Costs}

The operating and maintenance costs for the back end steps include costs due to chemicals, labor, and utilities required. These costs will largely be the same for each deployment strategy, with the exception of labor as the SMORE strategy runs these processes autonomously while the reference strategy requires operators to run the elution and regeneration processes. Additionally, the chemicals required for the back end will vary depending on the adsorbent used for the deployment.

\section{Disposal and Decommissioning (D\&D) Costs}

The D\&D costs associated for the back end are considered as they are for the adsorbent production process. That is, upon the project's completion, all facilities and 
equipment related to the back end must be decommissioned and/or disposed of. The $\mathrm{D} \% \mathrm{D}$ costs are considered to be $10 \%$ of the back end capital cost and are made payable on a yearly basis with the funds set aside for the D\&D of facilities earning interest in a sinking fund throughout the lifetime of the project. Selling the adsorbent for its backbone fiber as an alternative to the disposal of the adsorbent is also considered in this cost-analysis methodology. Specifically, the adsorbent could be recycled as a source of high-density polyethylene (HDPE), for which considerable knowledge into its recycle and reconstitution exists. Applications of the recovered HDPE include building and paving materials, or it may be directly recycled into the adsorbent fabrication process, thereby reducing the need to purchase virgin HDPE.

\subsection{Parameter Space}

This section describes the parameter space used in the cost analysis of the uranium from seawater production cost.

\subsubsection{Length of campaign}

As described in (2.2), the adsorbent uptake can be modeled as a one-side ligand saturation model and is a function of the immersion time, $t$, of the adsorbent in seawater. The immersion time is referred to as the length of campaign for the purposes of this cost analysis. Realistically, the campaign must be greater than 0 and should not exceed the time it takes to reach $95 \%$ of the saturation capacity of the adsorbent. This realistic limit is imposed because little gain will be realized by extending soaking times due to the asymptotic nature of (2.2).

\subsubsection{Temperature}

The uranium uptake of the adsorbent has been shown to be strongly affected by the seawater temperature, as discussed in 5.52 . In general, a higher temperature leads to both higher saturation capacity and half-saturation time, and thereby a greater adsorbent uptake. At present, the cost-analysis tool allows users to input a temperature for the deployment's entire lifetime.

\subsubsection{Degree of grafting}

The amidoxime functional groups of the adsorbent are attached to the HDPE adsorbent backbone through a process known as irradiation induced-grafting, as mentioned in chapter [1. The degree of grafting (DOG) is a measure of this addition of the amidoxime group to the HDPE backbone and is defined by

$$
D O G=\frac{W_{G}-W_{O}}{W_{O}} \times 100 \%,
$$


where $W_{G}$ is the weight of the grafted polyethylene and $W_{O}$ is the weight of the ungrafted polyethylene fibers. Although increasing the ratio of ligand to backbone improves the adsorbents uptake, it also increases the adsorbent production operating costs as it increases the grafting chemical consumption. Higher degrees of grafting lead to more available binding sites and a higher uranium capacity per mass of adsorbent, however experiments suggest that increasing the degree of grafting beyond $250 \%$ yields only marginal increase in the adsorbent's uranium uptake (Janke et al., 2014). Thus, the feasible domain for capacity is bounded such that it cannot exceed $250 \%$.

\subsubsection{Number of reuses}

The cost of uranium production from seawater is also affected by the number of times the adsorbent is used. As discussed in chapter 2, which each subsequent reuse, the chemical baths involved in the elution process degrade the uranium binding sites on the adsorbent. Hence, with each reuse, the adsorbent suffers degradation which compounds with use, as detailed by (2.12). The end of the adsorbent's lifetime is taken to be the point at which the accumulated degradation becomes so high that the marginal benefits of redeployment are outweighed by the marginal costs.

\subsubsection{Degradation}

The degradation of the adsorbent polymer directly influences the number of uses that are economically feasible. Initial experimental data suggested that the adsorbent would consistently suffer 5\% loss in uptake after each reuse (Sugo et al., 20101). However, more recent experimental data suggests that the adsorbent degradation rate

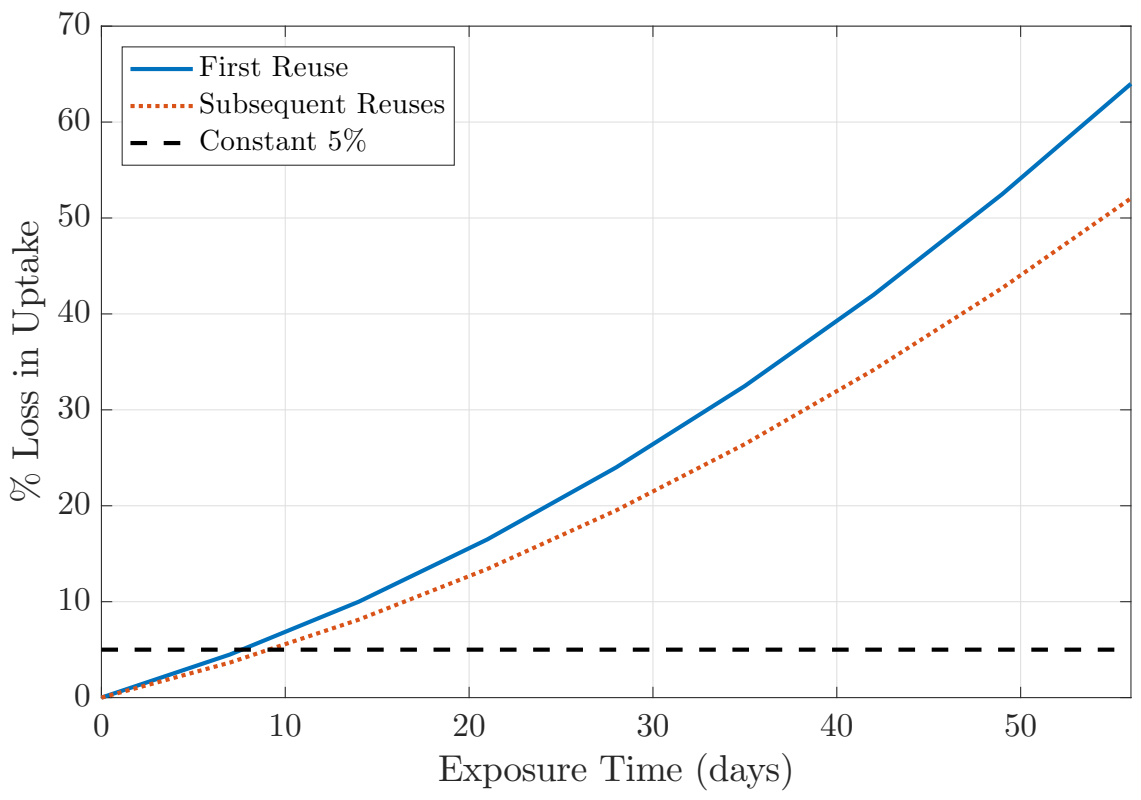

Figure 8-2: Loss in adsorbent uptake as a function of campaign length for the worst case degradation scenario, adapted from Byers and Schneider (2016b) 
is a function of campaign length and number of reuses, with the first reuse suffering the greatest loss in uptake (Kuo et al, 2015), as shown in figure 8-2 (Byers and Schneider, 2016b). Work by Pan et al. (2017) suggests that a bicarbonate elution process may remove uranium from the adsorbent with observed degradation rates of below $1 \%$ per reuse. Thus, the time dependent model for degradation will serves as an upper bound on the adsorbent degradation while the constant $5 \%$ loss in uptake provides the lower bound.

\subsubsection{Biofouling}

As previously mentioned in chapter 2 , biofouling has a significant affect on the adsorbent's ability to uptake uranium, namely decreasing the saturation capacity of the adsorbent. In the experiment by Park et al. (2016), the adsorbent fibers exposed to unfiltered seawater and kept in the presence of light suffered $30 \%$ loss in uptake as compared to control fibers. Additionally, this loss was consistent for all measured time-points. Therefore, the cost analysis model described in this chapter assumes the effects of biofouling with time to be negligible and a constant $30 \%$ loss in uptake is considered independent of campaign length.

Moreover, because biofouling is dependent on the temperature of seawater, in the absence of experimental data, a placeholder relationship between the temperature and biofouling of the adsorbent was derived and is integrated into the cost analysis by Byers (2015) to offset the unrealistic monotonic relationship between increasing temperature and uptake. In particular, while the uranium complexation with amidoxime fiber favors warmer waters (as described in chapter 2), it is likely that a competing feedback of increased biofouling also exists at these elevated temperatures. Detailed in chapter 叉, (2.16), relates the heterotrophic bacteria growth and ocean temperature, and is used in this cost analysis correlate the effects of biofouling to water temperature. The temperature dependent biofouling is applied only to the adsorbent saturation capacity because experiments have shown that temperature affects the adsorbent capacity but not the kinetics (Park et al., 2016). The details of the implementation of this dependence into the cost analysis model are described in Byers (2015).

\subsection{Reference Deployment Strategy}

The reference deployment strategy refers to the kelp-field like structure described first by Tamada et al. (2006) and later modified for economic improvements by Schneider and linder (2014). In this system, shown in figure 8-3, the adsorbent polymer is braided into buoyant $60 \mathrm{~m}$ long strands which are attached to metal chains that act to anchor the braids to the sea floor as well as hold rows of adsorbent braids together. After sufficient seawater exposure at the end of a campaign, the adsorbent braids are winched up by work boats which then transfer the braids to a mothership that houses the chemical bath for the elution of uranium from the polymers. The adsorbents are

then redeployed back to the field by the work boats. The adsorbents can be reused as 
(a)

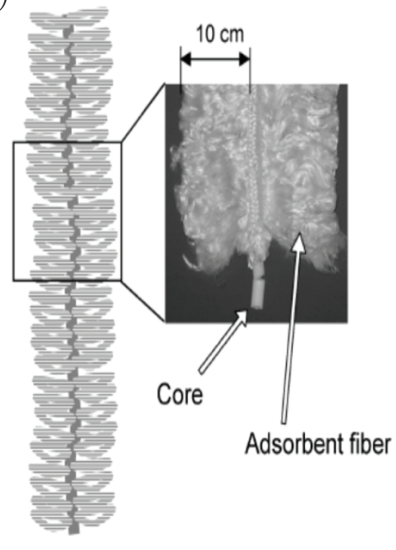

(b)

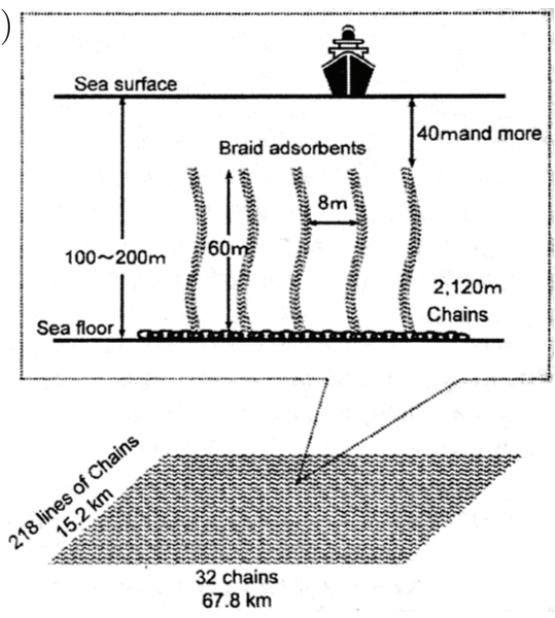

(c)

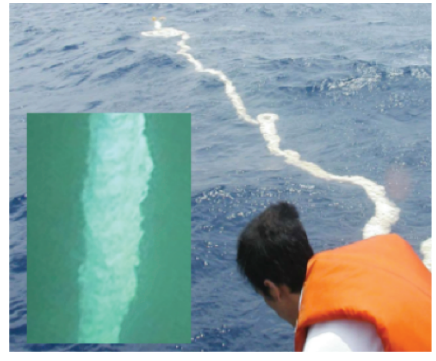

Figure 8-3: In the reference deployment strategy, the adsorbent is (a) braided into buoyant $60 \mathrm{~m}$ long lengths and (b) deployed across the seafloor. This strategy was (c) tested by the Japanese Atomic Energy Agency in the early 2000s (Tamada et al, $20(106)$

many times as is economically feasible, dependent upon the degradation they suffer with each deployment and elution cycle. Although this off-shore elution system offers improvement over the original method, there are still non-trivial expenses associated with the capital and operating costs of this system. The cost of uranium recovered by this means will serve as the reference system to which SMORE will be compared.

\subsection{SMORE Deployment Strategy}

The design analysis tool described in chapter 5 is employed in the cost analysis of a continuous version of SMORE in which the adsorbent net is constantly moving through the water column using a set of upper and lower rollers. Table $\mathbb{\nabla}$ describes the details of this system.

All support structures are considered to be made of 316 stainless steel. The capital cost of the SMORE structure was calculated primarily by the raw materials required to construct the device. Each harvester unit (i.e. each symbiotic wind turbine device) was sized to support and process the mass of adsorbent required to recover 1,200 tonnes of uranium per year (enough to supply a 5 GW nuclear reactor) from an entire wind farm consisting of 250 turbines.

The adsorbent production cost remained mostly unchanged from previous economic analyses with the kelp-filed deployment strategy. There was however a required cost to wind the adsorbent into koosh balls, fabricate the shells, and to construct the overall adsorbent nets suitable for deployment with this system.

The method of calculating elution and purification costs also remains mostly unchanged from previous analyses. While the elution of uranium off the adsorbent takes place at sea on the turbine, the necessary purification process was still assumed to 
Table 8.1: Details of continuous SMORE design used in cost analysis

\begin{tabular}{|l|l|l|}
\hline Parameter & Value & Notes \\
\hline System type & Stationary & \\
Current speed, $V_{c s}$ & $1 \mathrm{~m} / \mathrm{s}$ & Reasonable for boundary currents \\
Bottom current speed, $V_{c s, b}$ & $0.01 \mathrm{~m} / \mathrm{s}$ & \\
Depth to bottom current, $D_{c b}$ & $25 \mathrm{~m}$ & \\
Shell diameter, $d_{s}$ & $0.5 \mathrm{~m}$ & Input \\
Spacing between shells, $L_{s}$ & $0.1 \mathrm{~m}$ & Input \\
Shells per turbine, $N_{s t}$ & 16340 & Optimized by shell model (chapter [3) \\
Ball-chains lengths per roller & 10 & Input \\
System depth, $D_{\text {sys }}$ & $120 \mathrm{~m}$ & Input \\
Platform height above SWL, $H_{p}$ & $10 \mathrm{~m}$ & Input \\
Rollers, $N_{\text {roller }}$ & 4 & \\
Roller diameter, $d_{\text {roller }}$ & $2.3 \mathrm{~m}$ & \\
Roller inner diameter, $d_{\text {roller, }, \text { in }}$ & $2.1 \mathrm{~m}$ & \\
Roller length, $L_{\text {roller }}$ & $6.6 \mathrm{~m}$ & \\
Spacing between rollers, $L_{\text {sp,roller }}$ & $1 \mathrm{~m}$ & \\
Platform length, $L_{p t}$ & $2.74 \mathrm{~m}$ & \\
Rail radius, $r_{\text {rail }}$ & $0.25 \mathrm{~m}$ & \\
Groove radius, $r_{\text {groove }}$ & $0.35 \mathrm{~m}$ & \\
Wheels per rail, $N_{\text {wheel }}$ & 7 & Considering polyurethane wheels \\
Ball-chain rope diameter, $d_{r}$ & $0.016 \mathrm{~m}$ & \\
Ball-chain top tension, $T_{\text {top }}$ & $18620 \mathrm{~N}$ & \\
Ball-chain bottom tension, $T_{\text {bot }}$ & $17911 \mathrm{~N}$ & \\
Ball-chain max displacement, $y_{\text {max }}$ & $1.94 \mathrm{~m}$ & \\
Chemical agitation power, $P_{a g}$ & $12 \mathrm{~kW}$ & \\
Chemical heating power, $P_{\text {heat }}$ & $159.42 \mathrm{~kW}$ & \\
Chemical pumping power, $P_{\text {pump }}$ & $4.47 \mathrm{~kW}$ & \\
Roller motorizing power, $P_{\text {roller }}$ & $92 \mathrm{~kW}$ & \\
Total power required, $P_{\text {total }}$ & $546 \mathrm{~kW}$ & \\
\hline
\end{tabular}

take place on land. Therefore, the labor and facility costs for adsorbent elution are reduced. For this cost comparison, a bicarbonate elution process was considered and all costs incurred after the bicarbonate elution are calculated in the exact same way as in previous economic estimates (Schneider and Sachde, 20133; Byers, 2015; Byers et al, (2016).

\subsection{Results}

The parameters described in section 8.3 give a range of possibilities for the adsorbent's performance in open ocean conditions and hence the uranium production cost. The rate of adsorbent degradation and the affect of marine biofouling are used to characterize the best and worst case scenarios. Both of these uncertainties lead to a range 
Table 8.2: Input data used for cost-analysis

\begin{tabular}{|l|c|c|}
\hline Parameter & Best Case Value & Worst Case Value \\
\hline Adsorbent & \multicolumn{2}{|c|}{ AF1 } \\
\hline Temperature $\left({ }^{\circ} \mathrm{C}\right)$ & \multicolumn{2}{|c|}{20} \\
\hline Degree of Grafting (\%) & \multicolumn{2}{|c|}{ NaOH } \\
\hline Alkaline Solution & 0 & Worst Case \\
\hline Biofouling (\% loss in uptake) & 5 & \multicolumn{2}{|c|}{. } \\
\hline Degradation (\% loss per re-use) & \multicolumn{2}{|c|}{. } \\
\hline
\end{tabular}

Table 8.3: Optimized deployment parameters leading to the minimum achievable uranium production cost.

\begin{tabular}{|l|lll|lll|}
\cline { 2 - 7 } \multicolumn{1}{c|}{} & \multicolumn{3}{c|}{ Reference } & \multicolumn{3}{c|}{ SMORE } \\
\cline { 2 - 6 } \multicolumn{1}{c|}{} & Cost $(\$ / \mathrm{kgU})$ & Uses & Campaign & Cost $(\$ / \mathrm{kgU})$ & Uses & Campaign \\
\hline Worst Case & $\$ 870$ & 10 & 15 days & $\$ 593$ & 20 & 10 days \\
Best Case & $\$ 430$ & 13 & 45 days & $\$ 313$ & 20 & 50 days \\
\hline
\end{tabular}

of uranium production costs and are believed to represent the best and worst case scenarios, for the current technology. All performance scenarios were subjected to an optimization algorithm (Schneider and Sachde, 2013) used to find the deployment parameters, specifically length of campaign and number of adsorbent uses, that give rise to the minimum possible recovery cost. The details of the input parameters used in this cost analysis are described in table 8.2. The campaign length and number of adsorbent uses are determined using an optimization procedure (Byers, 2015) to find the lowest possible uranium production cost for the given set of inputs. The results for the cost optimization of both strategies is summarized in Table 8.3.

Figure $8-4$ shows the cost range for the best and worst case scenarios of both deployment strategies as a function of number of adsorbent uses. In both the best and worst case scenarios, the SMORE deployment strategy resulted in a lower recovery cost, in part due to a higher number of optimized uses, as seen by the shape of the curves in figure $8-4$. Additionally, the symbiotic scheme can sustain a longer campaign length as seen in table 8.3 since the cost of each deployment event is lower. The lower deployment capital cost favors a large field with longer soaking times as opposed to a smaller field with a higher turnover rate. This is especially evident in the case of the constant degradation rate as no penalty is suffered from longer deployments.

A cost breakdown for both deployment strategies is provided in figure $8-5$ for an intermediary case assuming no biofouling and the worst case, time-dependent degradation. After adsorbent production, the most significant cost contributor to the reference deployment strategy is the mooring and deployment cost; reducing this cost by means of the novel SMORE system presented here can thus have a substantial effect on the final uranium production cost. Given that for an intermediary deployment scenario the mooring and deployment comprise $37 \%$ of the production cost of uranium from seawater, SMORE could drastically reduce the estimated production cost. Additionally, significant reduction of the mooring and deployment cost which 


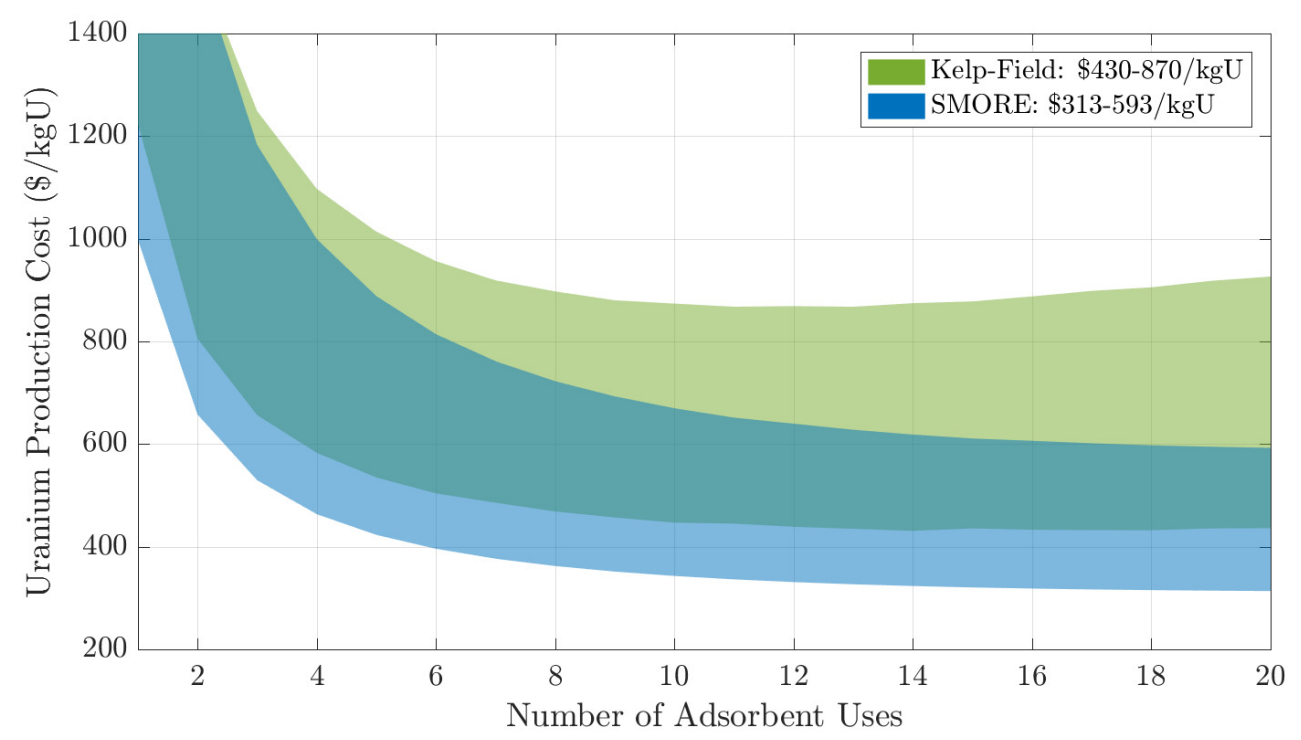

Figure 8-4: The range of costs for both deployment schemes as a function of number of adsorbent uses.

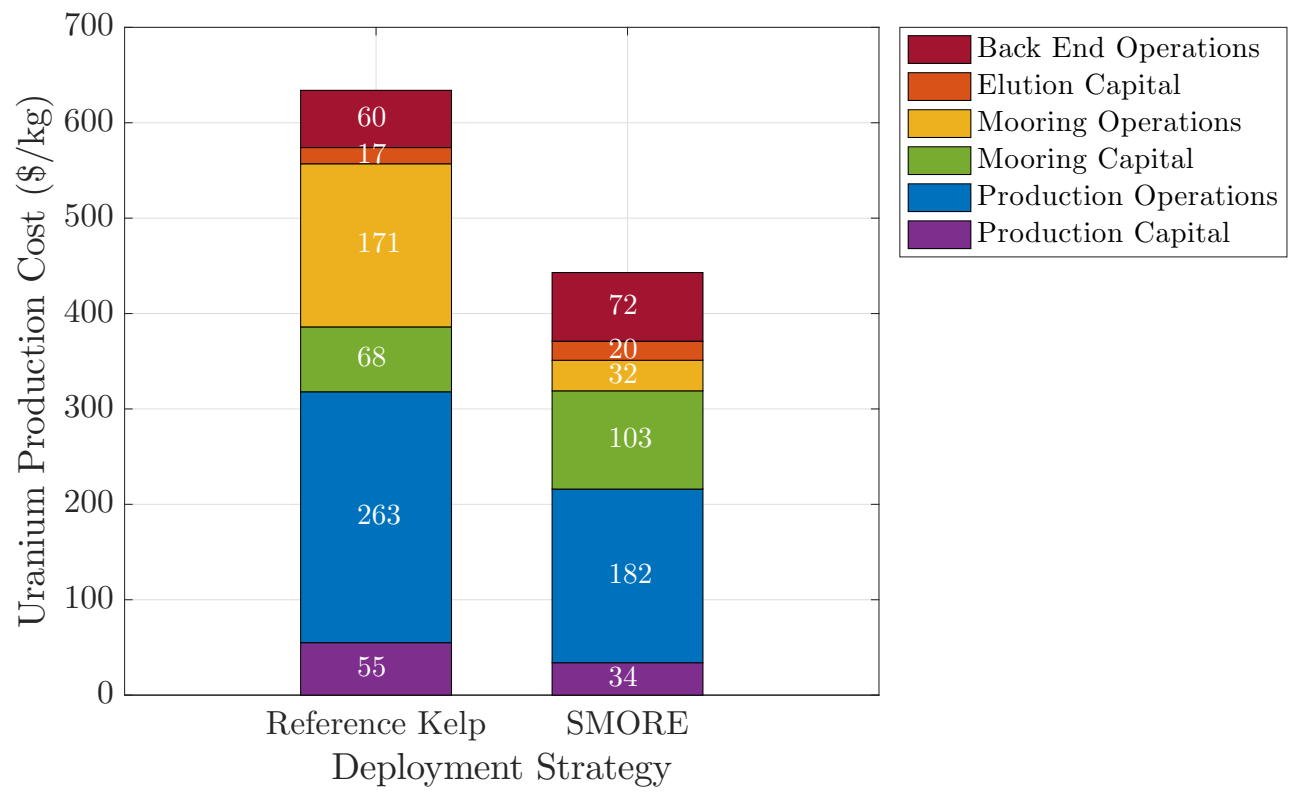

Figure 8-5: Breakdown of cost components contributing to the total cost of each deployment scheme for an intermediary case assuming no biofouling and worst case, time-dependent degradation.

allows for a much greater number of optimized uses, further improving uranium costs.

In this intermediary case, the reference deployment resulted in a cost of $\$ 634 / \mathrm{kg}$ $\mathrm{U}$ with a 15 day campaign length and 11 adsorbent uses, while the SMORE strategy resulted in a cost of $\$ 443 / \mathrm{kg} U$ for a campaign length of 10 days and 20 uses. As can be seen from the figure, the majority of the cost differences between the two strategies are due to the mooring capital and operation costs of each system. Specifically, the 


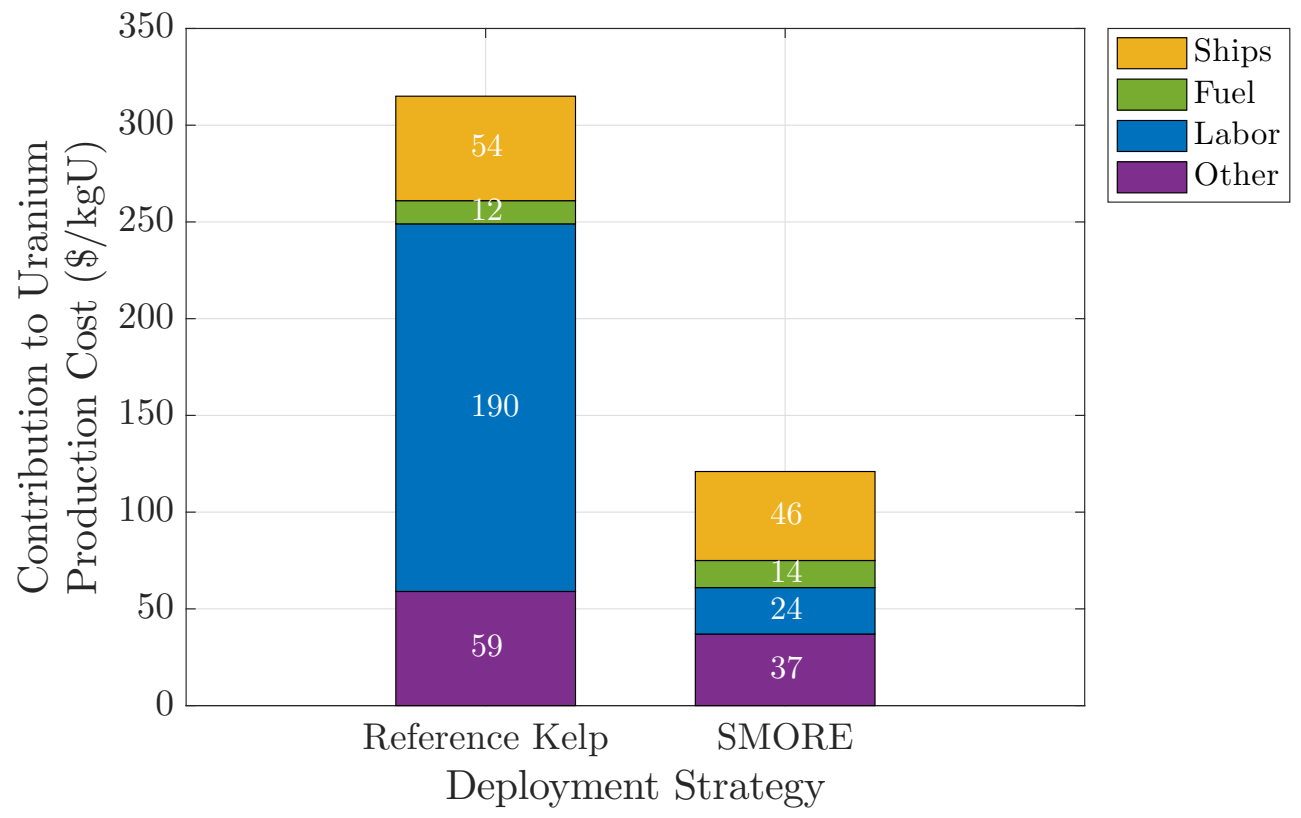

Figure 8-6: Some cost components contributing to the mooring capital and operating costs for each deployment strategy. The category labeled "Other" includes other operating consumables and contingencies.

SMORE strategy is able to reduce the mooring and deployment costs by over $43 \%$ so that they only contribute $\sim 30 \%$ to the overall uranium production cost.

Further examining some of the various components of the mooring capital and operating costs of both strategies, seen in figure [8-6, shows that the autonomous nature of the SMORE system results in significantly lower labor costs, which is responsible for the majority of the cost savings as compared to the reference deployment strategy. Specifically, the labor costs contribution to the mooring operating costs of the SMORE deployment are $87 \%$ less than those of the reference deployment strategy for this intermediary case.

\subsection{Conclusion}

This cost-analysis broke down the costs of recovering uranium from seawater into three major processes: (1) adsorbent production, (2) mooring and deployment, and (3) elution and regeneration. As can be seen from this study, the mooring and deployment costs can be drastically reduced by coupling the recovery of uranium from seawater with offshore wind power generation. This is especially impactful if the best case scenario regarding adsorbent performance can be realized, meaning oceanic biofouling can be mitigated to realize negligible effects on uptake and adsorbent degradation rate can be restored to previously observed levels.

Specifically, the SMORE deployment strategy has the potential to decrease the uranium production cost by drastically decreasing the mooring and deployment costs. In the design studied here, for an intermediary case considering no biofouling and time 
dependent degradation, the SMORE strategy achieves an $87 \%$ reduction in the labor costs associated with the deployment, directly translating to a $43 \%$ reduction in the mooring and deployment costs and an overall cost reduction of $30 \%$ in the production cost of uranium from seawater.

Of the major processes, the next largest cost driver that remains is the adsorbent production cost. In SMORE strategy, this process now accounts for over $48 \%$ of the uranium production cost. These costs can be reduced by either increasing the adsorbent capacity (and therefore reducing the amount of adsorbent required for a deployment), reducing the affect of biofouling on the adsorbent (and thereby increasing the time the adsorbent can be used for), decreasing the degradation of the adsorbent after each elution cycle (thus allowing for increased reuse of the adsorbent), or reducing the overall cost of adsorbent production. As shown figure 8-7, which examines the cost components related to the adsorbent production for this intermediary case, the major cost drivers of the adsorbent production include the material costs (namely hydroxylamine $\mathrm{HCl}$ which is required for the irradiation-induced grafting process of the amidoxime ligand onto the radical sites on the polyethylene backbone), electricity costs, and maintenance.

Although this cost analysis accounted for the effect of biofouling reducing the uptake of uranium by the adsorbent, it does not consider how this biofouling will affect other steps in the uranium extraction process. For instance, growth on the adsorbents may increase the amount of chemicals required for the elution and regeneration processes per tonne of adsorbent, thereby increasing the cost. Additionally, because

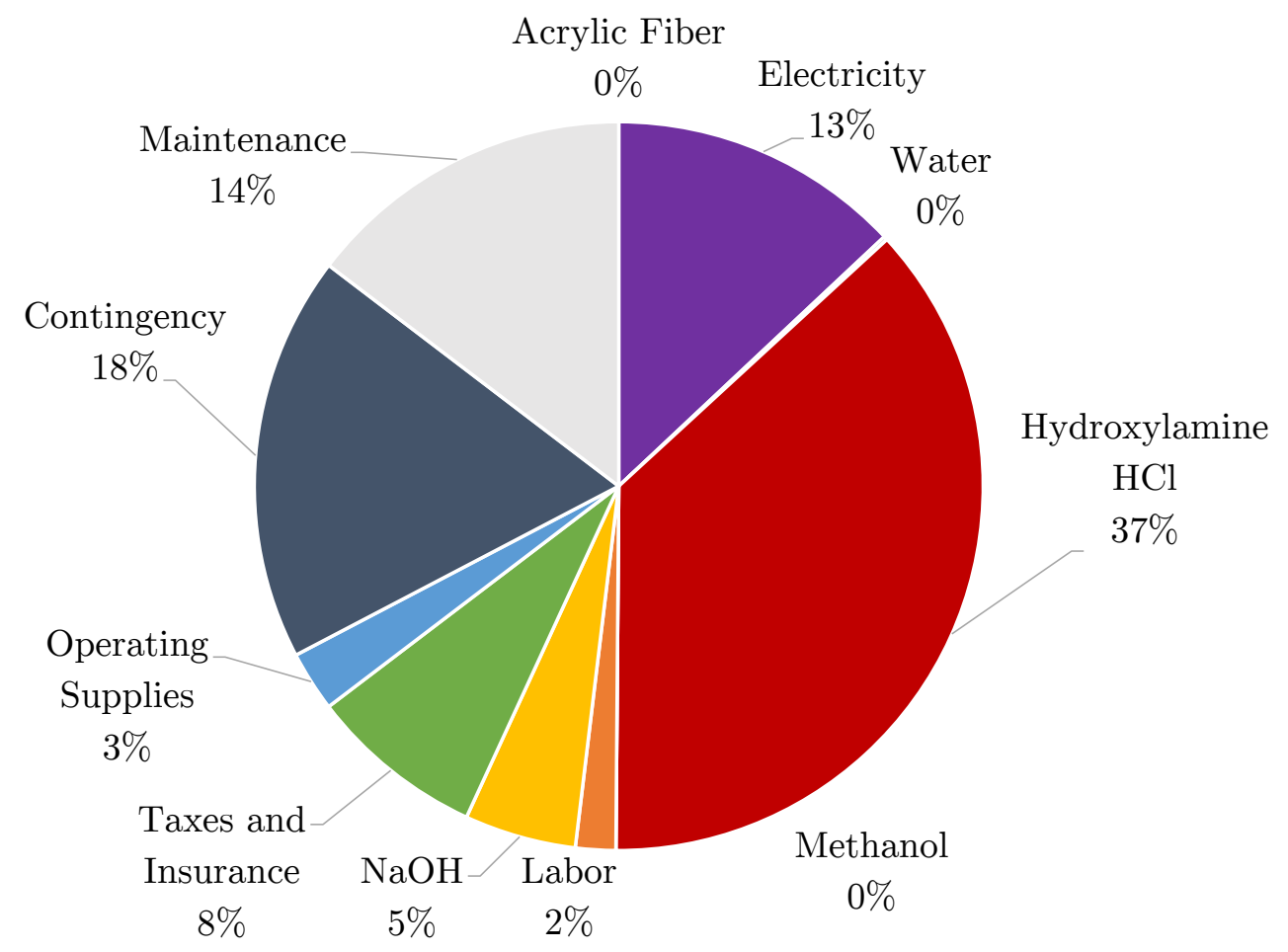

Figure 8-7: Breakdown of adsorbent production costs. 
the chemical processing will be done autonomously at sea, there may also be other marine debris that entire the chemical tanks during elution and regeneration which may increase the amount of chemicals needed for these processes even more. A future cost analysis of a symbiotic system to harvest uranium from seawater should account for these additional chemical requirements. 


\section{Chapter 9}

\section{Seawater Uranium Suitability of Harvesting Index (SUSHI)}

This chapter details the Seawater Uranium Suitability of Harvesting Index (SUSHI) and the graphical user interface (GUI) developed to examine worldwide feasibility of harvesting uranium from seawater using a symbiotic device attached to an offshore wind turbine.

\subsection{Motivation}

The Suitability Index is defined to be how appropriate a location is to the harvesting of uranium from seawater by way of a symbiotic system attached to an offshore wind turbine support structure. The index is comprised of the wind power potential of various sites around the globe as well as the adsorbent capacity, which is dependent on temperature and light, in those regions. This number is then normalized by that of a similar deployment strategy off the coast of southern Japan, a location where many previous studies of seawater uranium harvesting have taken place (Seko et al,, 20(1)3; ?; Tamada et al., 2006); Tamada, 20(1).9).

\subsection{Incorporated factors}

\subsubsection{Wind Power}

Of importance in siting an offshore wind turbine is the power potential at that location. Wind power is proportional to the cube of the wind speed by:

$$
P=\frac{1}{2} \eta \rho A v^{3}
$$

where $P$ is the wind power, $\eta$ is the efficiency of the turbine, $\rho$ is the density of air, $A$ is the rotor area of the wind turbine, and $v$ is the speed of the wind perpendicular to the turbine rotor. In this model, it is assumed that the only variable in the turbine design is its site offshore, therefore in (‥]), only $v$ is changing from location to location. 


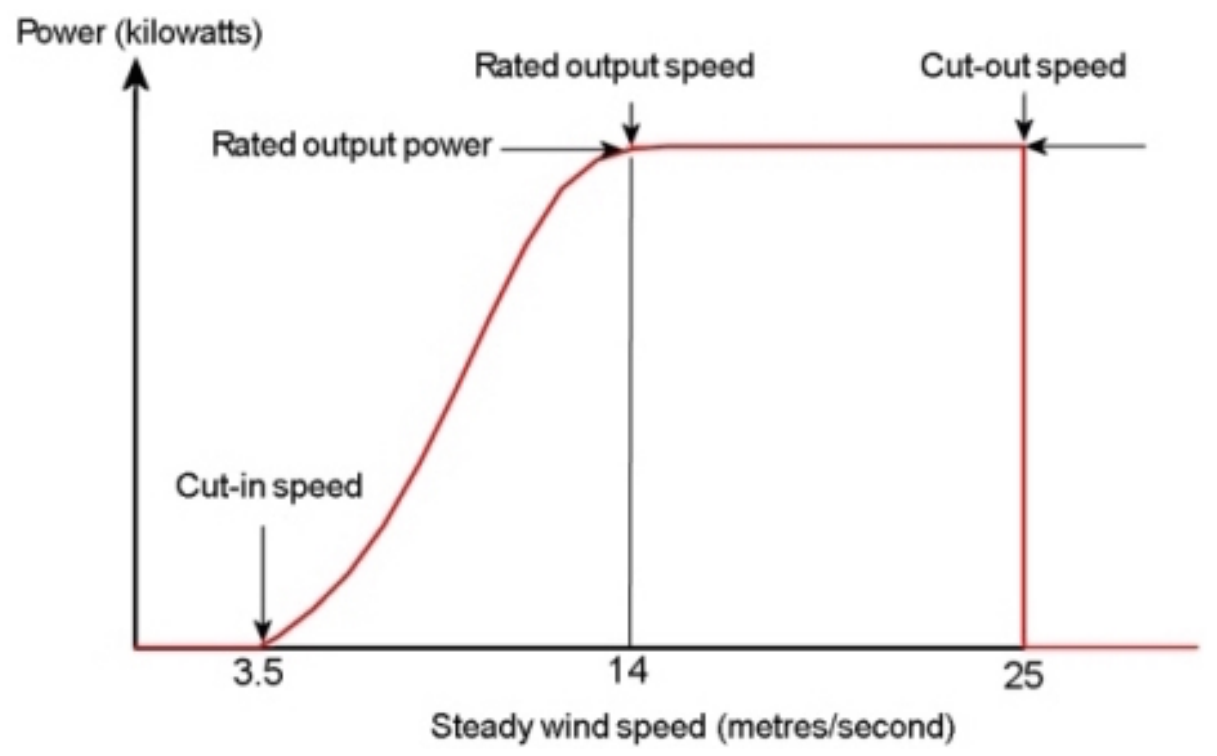

Figure 9-1: Typical wind turbine power output curve (PelaFlow Consulting, 2008).

The ocean surface wind dataset available only provides the wind speeds near the ocean surface, $\sim 10 \mathrm{~m}$ high. Offshore wind turbines operate at much higher heights, $80 \mathrm{~m}-100 \mathrm{~m}$. Therefore, the wind speed near the surface was related to the wind speed at an arbitrary height by

$$
v_{r}=v_{a}\left(\frac{z_{r}}{z_{a}}\right)^{\nu},
$$

where $v_{r}$ is the unknown wind speed at height $z_{r}, v_{a}$ is the measured wind speed at height $z_{a}$, and $\nu$ is the power law wind speed shear exponent (E्lliot et al., 1987). For this calculation, $\nu$ was chosen to be 0.11 based on validation with updated offshore wind maps and other analyses of offshore wind resources that indicate that the shear exponent is most often in the range from 0.08 to 0.14 for the offshore regions of the United States (Schwartz et al, 2010).

Given that the typical wind turbine power output curve is not constant, as shown in figure 9-1, the minimum and maximum operation speeds of the wind turbine are left as inputs to SUSHI. These speeds correspond to the cut-in speed and cut-out speed shown in figure [-1], respectively. To account for this power curve, $v_{r}^{\prime}$ is defined to be the resulting dataset after adjusting $v_{r}$ from (9.2) to remove any speeds below the cut-in speed and above the cut-out speed of the wind turbine. Finally, since the wind power is proportional to the cube of the wind speed by (प.1), a matrix, $M_{v}$, of the wind speeds cubed relating to each location on the globe was defined from the adjusted wind speed at the turbine height, $v_{r}^{\prime}$ :

$$
M_{v}=v_{r}^{\prime 3}
$$




\subsubsection{Adsorbent capacity}

The adsorbent capacity is taken to be a function of temperature, as described in chapter 2]. Depending on the type of adsorbent chosen for analysis $(38 \mathrm{H}, \mathrm{AF} 1$, or AI8), the adsorbent capacity is determined by equation ([2.13), ([2.14), or (2.15). As a result of this dependence of adsorbent capacity on seawater temperature, a matrix of adsorbent capacity at all locations on the globe, $M_{k}$, is defined using ([.].3), ([.]4), or (2.15), and a specific campaign length, $t$. The total uranium adsorbed per tonne adsorbent after $n$ uses is given by equation ([2.2) which is computed for a specific temperature, campaign length, number of uses, and degradation per cycle.

\subsection{Suitability Index}

At present, the Suitability Index is a function of the potential wind power and adsorbent capacity of various locations across the globe. Additionally, a reference location is chosen to aid in the comparison of various potential seawater uranium sites. Using the resulting matrix of wind speeds at the turbine height cubed, $M_{v}$ and the adsorbent capacity, $M_{k}$ at all locations around the globe the suitability index is defined as

$$
S I=\left(\frac{M_{v}}{v_{0}^{3}}\right)^{\alpha}\left(\frac{M_{k}}{k_{0}}\right)^{\beta}
$$

where $v_{0}$ is the wind speed at the reference location and $k_{0}$ is the adsorbent capacity at the reference location. In the SUSHI tool, $\alpha=\beta=1$, that is the wind power potential and adsorbent capacity of the region are weighted equally. The reference location is taken to be $128.5^{\circ} \mathrm{E}, 26.5^{\circ} \mathrm{N}$, a location off the coast of southern Japan, where many previous experiments of uranium harvesting adsorbents have been conducted (Seko et al., 2003); ?; Tamada et al., 2006); Tamada, 200). $)$.

Resulting values of the Suitability Index (SI) can range from 0 to any positive number. Values equal to 1 indicate places where uranium harvesting would be as suitable as offshore of the southern coast of Japan. Sites with SI values less than 1 indicates areas where uranium harvesting is less suitable and locations with SI values greater than 1 are more suitable for uranium harvesting than off the coast of southern Japan.

\subsection{Datasets}

This section provides details about the datasets utilized by the latest version of the SUSHI tool. All datasets are publicly available and were provided by either NASA or NOAA. 


\subsubsection{Ocean surface winds}

The ocean surface winds dataset was retrieved from the NASA Jet Propulsion Laboratory Physical Oceanography Distributed Active Archive Center (PODAAC). The specific dataset is the "SeaWinds on QuikSCAT Level 3 Surface Wind Speed for Climate Model Comparison" that was produced by the QuickSCAT Project. Launched in June of 1999, QuickSCAT was designed to be a "quick recovery" EOS satellite mission to fill the gap of global ocean surface wind vector observations which resulted from the unexpected failure of NSCAT in June of 1997.

This dataset was then utilized to determine the wind speed at the turbine height, as described in section 9.2 .1 . The following are the details of the QuickSCAT dataset for the ocean surface winds:

- Spatial Resolution: 1 degrees (Latitude) x 1 degrees (Longitude)

- Longitude Range: $180^{\circ} \mathrm{W}$ to $180^{\circ} \mathrm{E}$

- Latitude Range: $89.6^{\circ} \mathrm{S}$ to $89.6^{\circ} \mathrm{N}$

- Temporal Resolution: 1 month

- Time Span: 1999-Aug-01 to 2009-Oct-31

- Variables: sfcWind - Near-surface wind speed (10m above ocean surface)

- Units: $\mathrm{m} / \mathrm{s}$

- Plotted Variable: Long term mean (1999-2009) wind speed for input month or the average over all months

More information about the dataset can be found on the PODAAC website:

http://podaac.jpl.nasa.gov/dataset/QSCAT_L3_SFC_WIND_SPEED_1DEG_1MO

\subsubsection{Sea surface temperature}

The sea surface temperature dataset was derived from the NOAA Earth Systems Research Laboratory. It is titled "NOAA Optimum Interpolation (OI) Sea Surface Temperature (SST) V2" and includes long term mean information from the years 1971 to 2000. The data was produced using both in situ and satellite data (Reynolds et al., 2(1)2).

This dataset was utilized to determine the adsorbent capacity at various sites across the globe. The details of the dataset are as follows:

- Spatial Resolution: 1 degrees (Latitude) x 1 degrees (Longitude)

- Longitude Range: $0^{\circ} \mathrm{E}$ to $360^{\circ} \mathrm{E}$

- Latitude Range: $89.5^{\circ} \mathrm{S}$ to $89.5^{\circ} \mathrm{N}$

- Temporal Resolution: 1 month

- Time Span: 1971 to 2000

- Variables: sst - sea surface temperature

- Units: ${ }^{\circ} \mathrm{C}$

- Plotted Variable: Long term mean (1971-2000) temperature for input month or the average over all months

More information about the dataset can be found on the NOAA website: 


\subsection{GUI Presentation}

This section presents a detailed explanation of the SUSHI graphical user interface (GUI) along with a step-by-step example to demonstrate how to run the code.

\subsubsection{GUI Presentation}

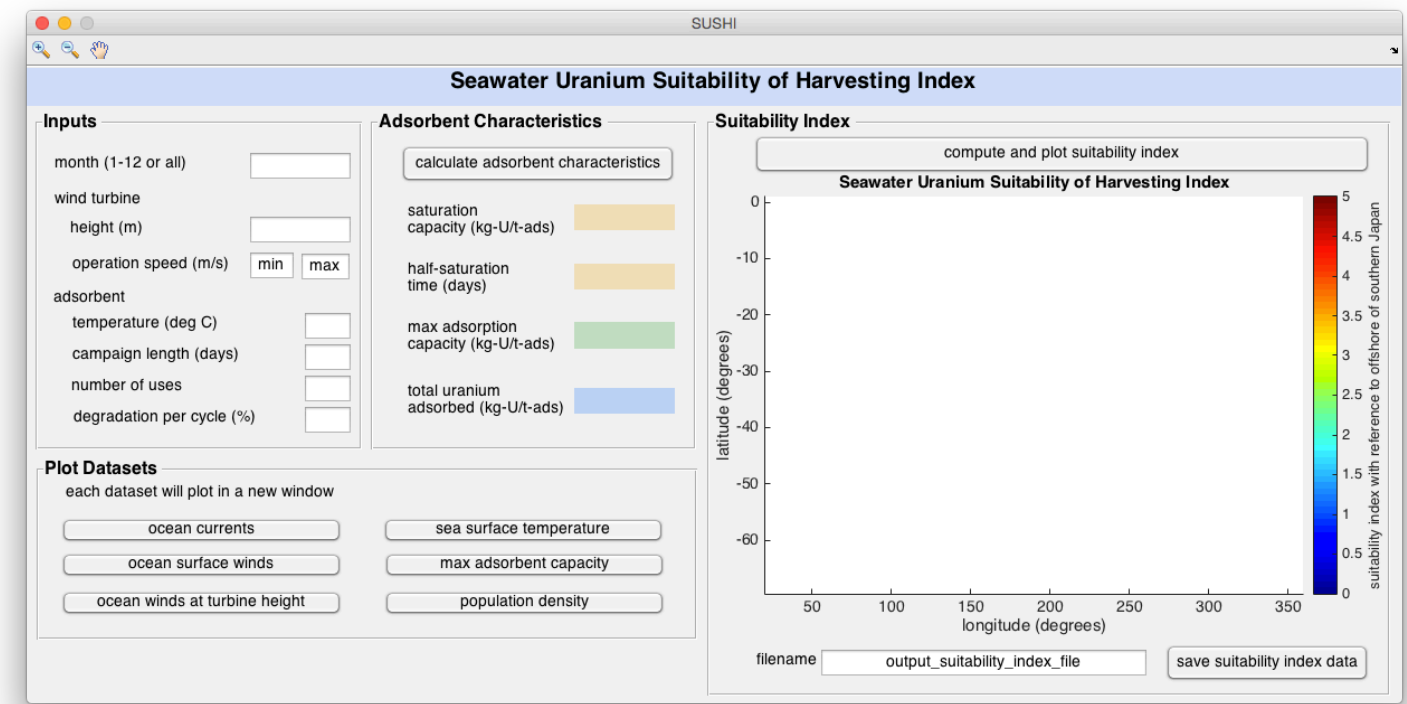

Figure 9-2: SUSHI GUI

The SUSHI package provides a GUI that combines all the datasets necessary for determining the suitable global sites for seawater uranium harvesting. A screenshot of the GUI is provided in figure Q-2]. The steps detailed below describe what the user must do in order to get started using the GUI.

Step 1 defines the inputs required by the tool. Step 2 allows the user to plot the datasets utilized by the calculation. Step 3 then utilizes the user input parameters and dataset variables to determine the suitable sites for the harvesting of uranium from seawater. More details on each specific element to provide to the GUI are listed below.

\section{Step 1: Inputs}

- Month: The month of interest must be entered into the program using the values 1-12 corresponding to the months of the year, or the term "all" to average the data over all months. This input is required due to the high seasonality of 
global sea surface temperatures and winds. If no value is entered, the default month value of 6 (corresponding to June) is utilized by the tool.

- Wind Turbine Height: The height in meters of the wind turbine is required by the tool. This value is used order to determine the wind speeds at the turbine's height. If no value is entered, a standard height of $90 \mathrm{~m}$ is used.

- Wind Turbine Operation Speed: The minimum and maximum operation speeds of the wind turbine must be entered in $\mathrm{m} / \mathrm{s}$. These values are used to determine sites suitable for offshore wind farms. If no values are entered, reference values for the minimum and maximum speeds of $3.5 \mathrm{~m} / \mathrm{s}$ and $25 \mathrm{~m} / \mathrm{s}$ are used, respectively.

- Adsorbent Temperature: The temperature in ${ }^{\circ} \mathrm{C}$ at which you would like to determine the maximum adsorption capacity. If no value is given, $20^{\circ} \mathrm{C}$ is utilized.

- Campaign Length: The time in days that the adsorbent is submerged in seawater. If no value is given, a default campaign length of 30 days is used.

- Number of Uses: The number of times the adsorbent is eluted in order to harvest uranium before the adsorbent is discarded. If no value is given, the number of uses is taken to be 12 .

- Degradation per Cycle: The adsorbent degradation in percentage after each use due to the acid elution bath. If no value is given, the degradation per cycle is taken to be $5 \%$.

\section{Step 2: Adsorbent Characteristics}

It might be of interest to the user to know the characteristics of the adsorbent given the adsorbent inputs in Step 1. The characteristics computed are

- Saturation Capacity: The saturation capacity of the adsorbent in $\mathrm{kg}-\mathrm{U} / \mathrm{t}$ ads. This value is temperature dependent and is computed using the reference temperature inputted in Step 1.

- Half-Saturation Time: The half-saturation time of the adsorbent in days. This value is temperature dependent and is computed using the reference temperature inputted in Step 1.

- Maximum Adsorption Capacity: The maximum adsorption capacity in kg$\mathrm{U} / \mathrm{t}$-ads of the adsorbent as defined using a one-site ligand model from values inputted in Step 1. This model is described in detail in chapter 2 .

- Total Uranium Adsorbed: The total uranium adsorbed in kg-U/t-ads for the lifetime of an adsorbent using the inputs given in Step 1. 


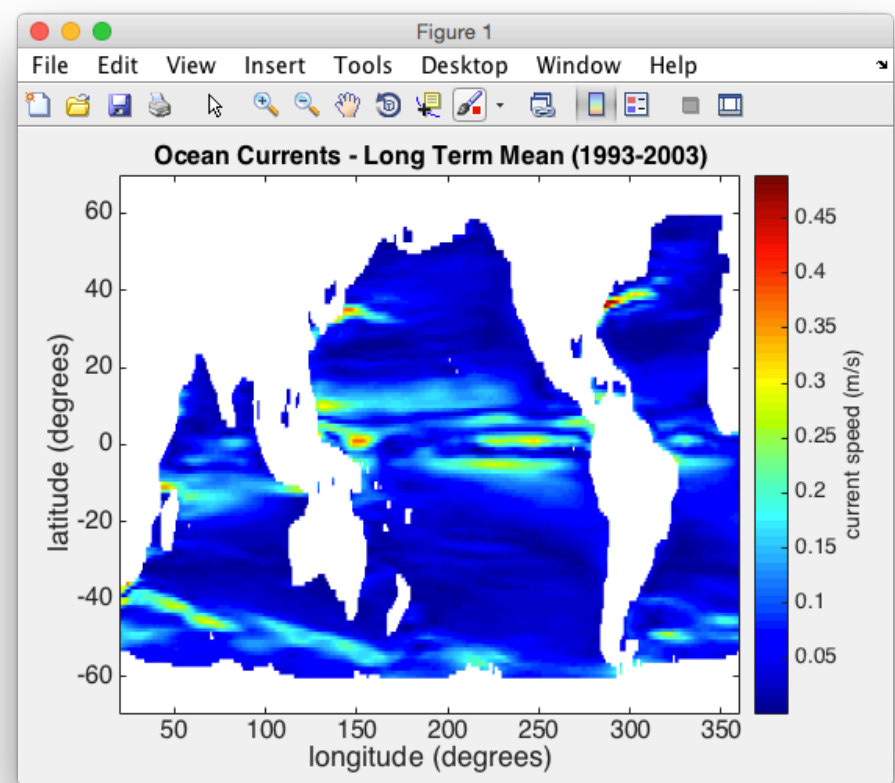

Figure 9-3: Typical ocean currents dataset plot.

\section{Step 2: Plot Datasets}

In this step, the user can preview the global datasets utilized by the tool in computing the Suitability Index. Each dataset selected will be shown in a new window. More information about each dataset is described in section 4.4 . The global region plotted is between $20.5^{\circ} \mathrm{E}-359.5^{\circ} \mathrm{E}$ and $69.5^{\circ} \mathrm{S}-69.5^{\circ} \mathrm{N}$ because these are the common locations at which all datasets are defined.

- Ocean Currents: The long term mean ocean currents in m/s from 1993-2003 are displayed. Figure $4-3$ shows the typical output. Recent work Ladshaw et al. (2017) has shown that linear flow rates as low as $4 \mathrm{~cm} / \mathrm{s}$ are more than enough to provide enough water flow to make a seawater uranium harvester feasible, therefore the ocean currents are not incorporated into the present Suitability Index. However, should seawater uranium harvesting systems be limited in the currents required, this dataset could easily be incorporated into an updated Suitability Index. It also expands the potential for SUSHI to consider additional symbiotic uses for the offshore structure that supports the turbine such as underwater turbines for power generation or perhaps even aquaculture systems that rely on currents.

- Ocean Surface Winds: Selecting this option plots the long term mean of the ocean surface winds in $\mathrm{m} / \mathrm{s}$ for the month selected or the average for all months (in the case that the user inputs "all") from 1999-2009. Figure Q-4 displays 
the result for the default month of June. This dataset is used to determine the ocean surface winds at the height of the wind turbine.

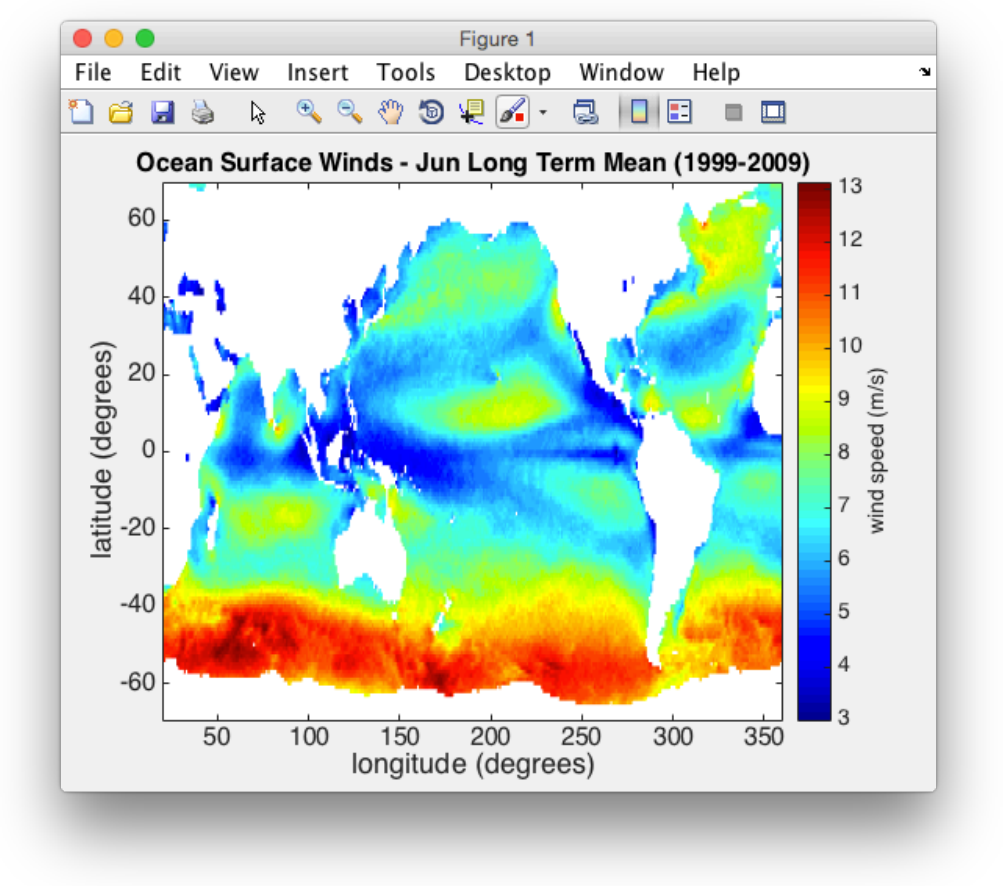

Figure 9-4: Typical ocean surface winds dataset plot.

- Ocean Winds at Turbine Height: This option plots the long term mean of the ocean winds at the entered turbine height in $\mathrm{m} / \mathrm{s}$ for the month selected or the average for all months (in the case that the user inputs "all") from 19992009 .

- Sea Surface Temperature: The long term mean sea surface temperature in ${ }^{\circ} \mathrm{C}$ from 1971-2000 for the desired month or the average for all months (in the case that the user inputs "all") is plotted. A typical output plot is displayed in figure $4-5$. This dataset is utilized to determine the likely adsorbent capacity for sites around the globe.

- Maximum Adsorbent Capacity: The adsorbent capacity in g-U/kg-ads is plotted for various locations around the globe. This dataset is derived from the global sea surface temperature dataset as well as the user inputs for reference adsorbent capacity and temperature.

\section{Step 4: Suitability Index}

The values entered in Step 1 are used here in Step 4 to determine the Suitability Index of various locations around the globe for harvesting uranium from seawater. 


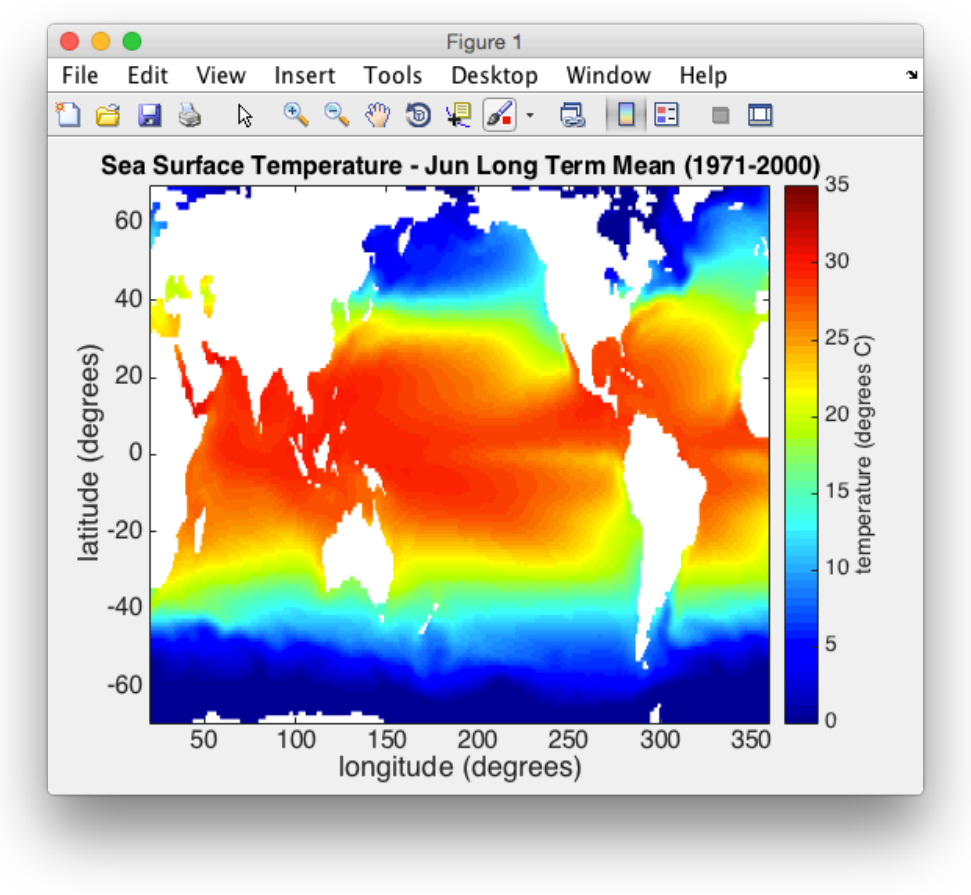

Figure 9-5: Typical ocean sea surface temperature plot.

The details of the calculation are described in section 29.3. The Suitability Index indicates how a potential uranium harvesting site compares to the region off the coast of Southern Japan, a location where many previous studies regarding seawater uranium harvesting were conducted (Seko et al, 20013; ?; Tamada et al, 2006; Tamada, 20019).

Pressing the "compute and plot suitability index" button will populate the axes below with the Suitability Index values of sites around the world. An index of 1 indicates that the location is similar to the region offshore of Southern Japan. An index less than 1 corresponds to a location that is not as suitable for seawater uranium harvesting as the region off the coast of Southern Japan, and an index greater than 1 signifies that the area is more suitable. The suitability of a location is determined by the adsorbent capacity as well as the power of the wind turbine due to ocean winds at that location.

Figure [-6] shows a screenshot of the typical output from the SUSHI tool. The tools in the upper left corner of the SUSHI GUI (see figure 2-2) allow the user to zoom in, out, and pan to move through the resulting image.

\subsubsection{Data output}

In addition to the Suitability Index plot, the SUSHI tool also provides the option to export the resulting suitability index data for further analysis. To do this, the user must define a filename in the "output_suitability_index_file" box and then click "save suitability index data." If no filename is given, the file will be saved as: 


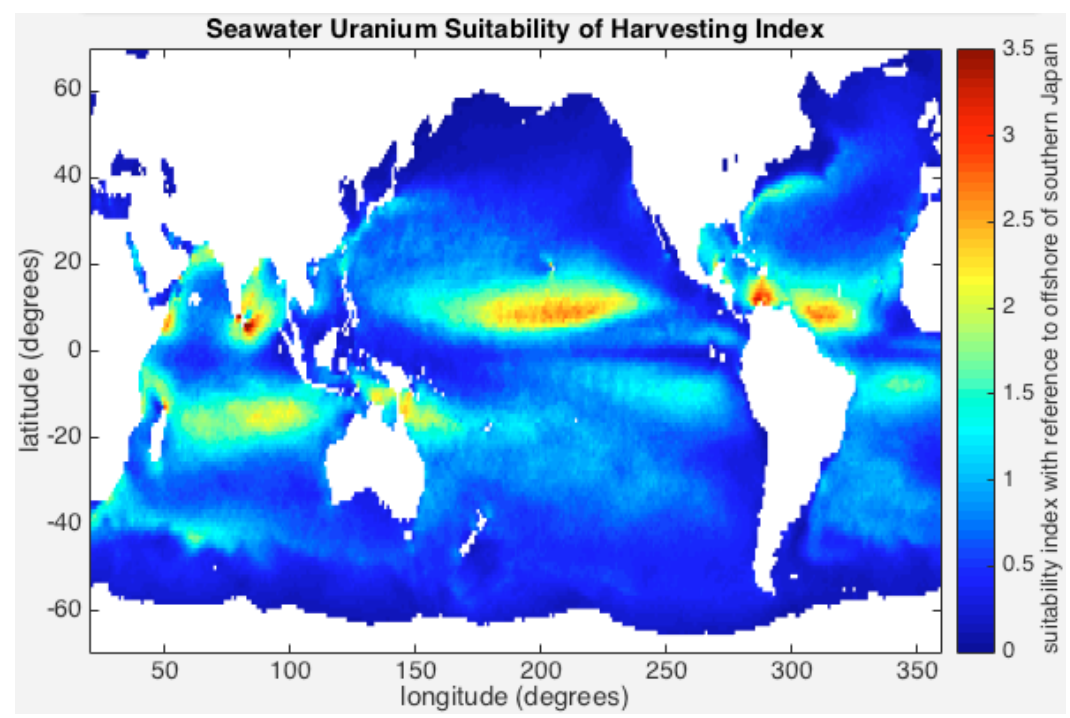

Figure 9-6: Typical suitability index plot.

"SI-month $\#_{M-} \mathrm{t} \#_{T-\mathrm{h}} \#_{H-\mathrm{v}} \#_{V_{1}}-\#_{V_{2}} \cdot$ mat"

where

- $\#_{M}$ corresponds to the month value

- $\#_{T}$ is the campaign length (days)

- $\#_{H}$ is the wind turbine height

- $\#_{V_{1}}$ is the minimum wind turbine operation speed

- $\#_{V_{2}}$ is the maximum wind turbine operation speed

The output data file contains the following variables:

- 'lon': an array of the longitude at which the index is defined

- 'lat': an array of the latitude at which the index is defined

- 's_index': a matrix of the Suitability Index at each of the locations defined

\subsubsection{Reminders: DOs and DONTs}

In order to properly utilize the SUSHI tool, here are some things to consider.

- The month value must be between 1 (corresponding to January) and 12 (corresponding to December), or "all" for an average over all months.

- The wind turbine height must be in meters, m.

- The wind turbine operation speeds must be in $\mathrm{m} / \mathrm{s}$.

- The maximum wind turbine operation speed cannot be less than the minimum wind turbine operation speed.

- The reference adsorbent temperature must be in ${ }^{\circ} \mathrm{C}$.

- The degradation per cycle must be in percent (i.e. $5 \%$ degradation must be inputted as "5". 


\subsubsection{An example}

Presented here is an example of how to use the GUI for a test-case study. For this study, the following inputs were utilized:

- Month: 6

- Wind turbine height: $90 \mathrm{~m}$

- Wind turbine operation speeds: $\min =3.5 \mathrm{~m} / \mathrm{s}, \max =25 \mathrm{~m} / \mathrm{s}$

- Reference adsorbent temperature: $20^{\circ} \mathrm{C}$

- Campaign length: 30 days

- Number of uses: 12

- Degradation per cycle: $5 \%$

These are also the default values used by the tool in the event that the user does not provide any inputs.

Figure $9-7$ shows a screenshot of the GUI after this step.

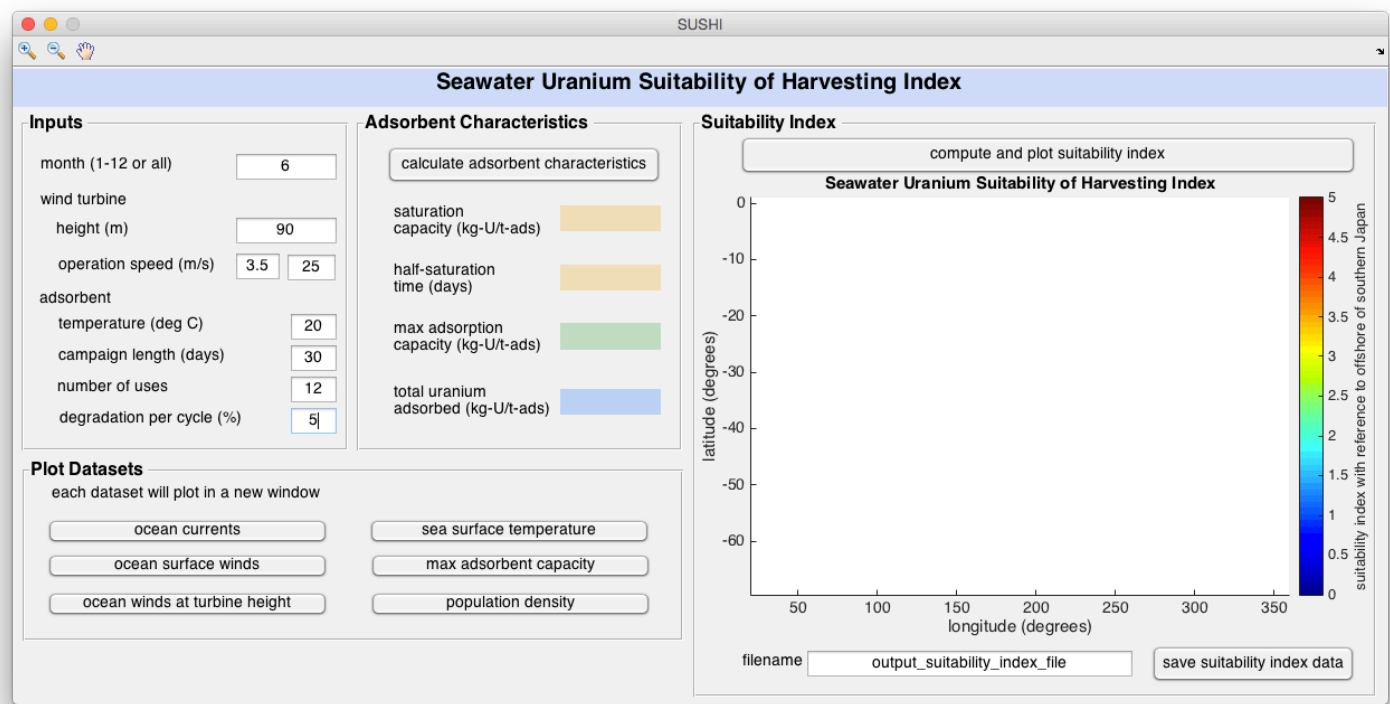

Figure 9-7: Screenshot of the GUI after the example inputs are entered.

Selecting any of the datasets will result in a new window in which they are displayed. For this example, "ocean winds at turbine height" and "maximum adsorbent capacity" are selected. Figure $9-8$ shows a screenshot of the output plots for these selections.

Next, click "compute and plot suitability index" to visualize the suitability of various ocean sites for seawater uranium harvesting based on the previous inputs. Additionally, a name for the output file is selected to save the suitability index data. The GUI screen at this step is shown in figure 2-9. 


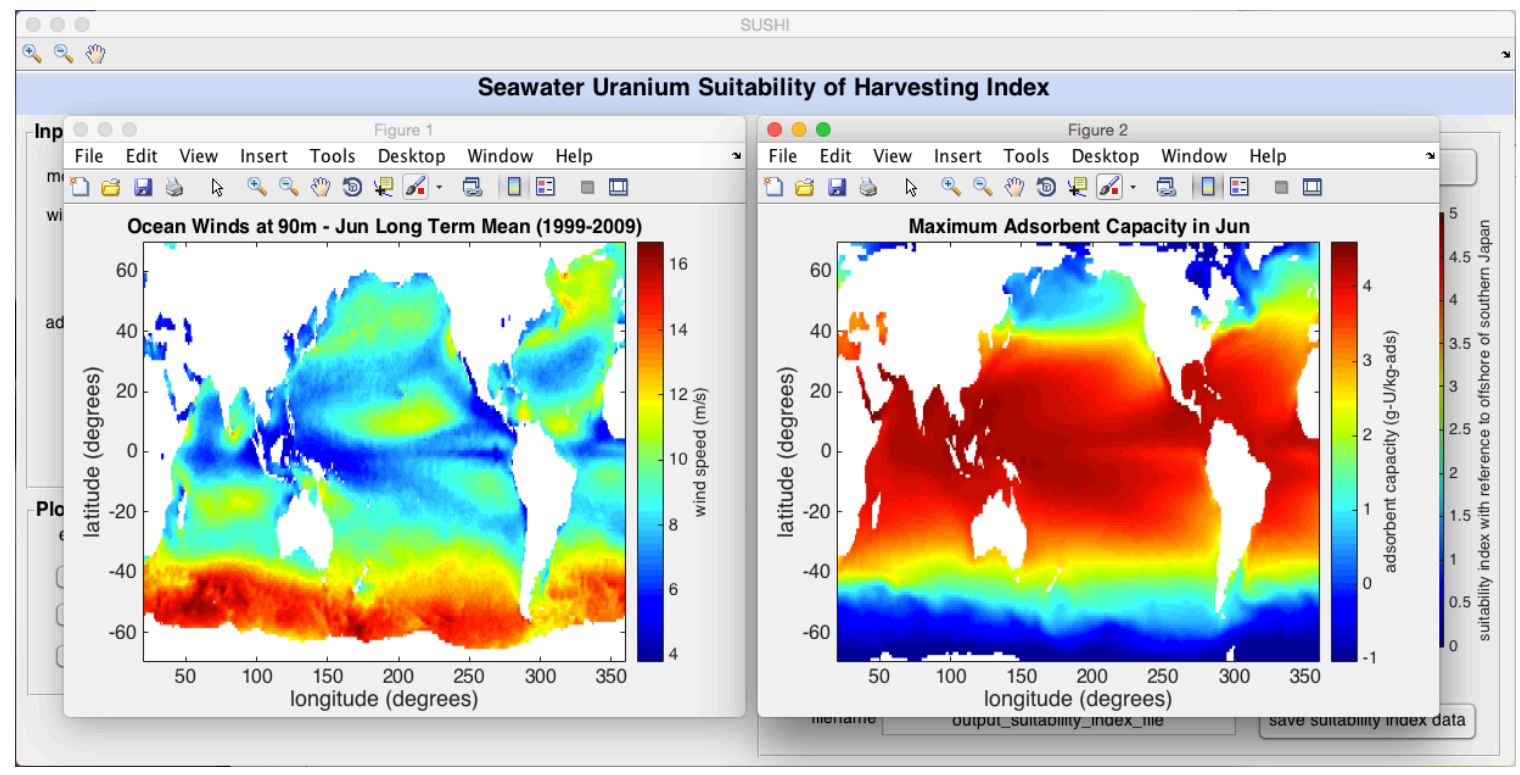

Figure 9-8: Screenshot of the GUI after selected datasets are plotted.

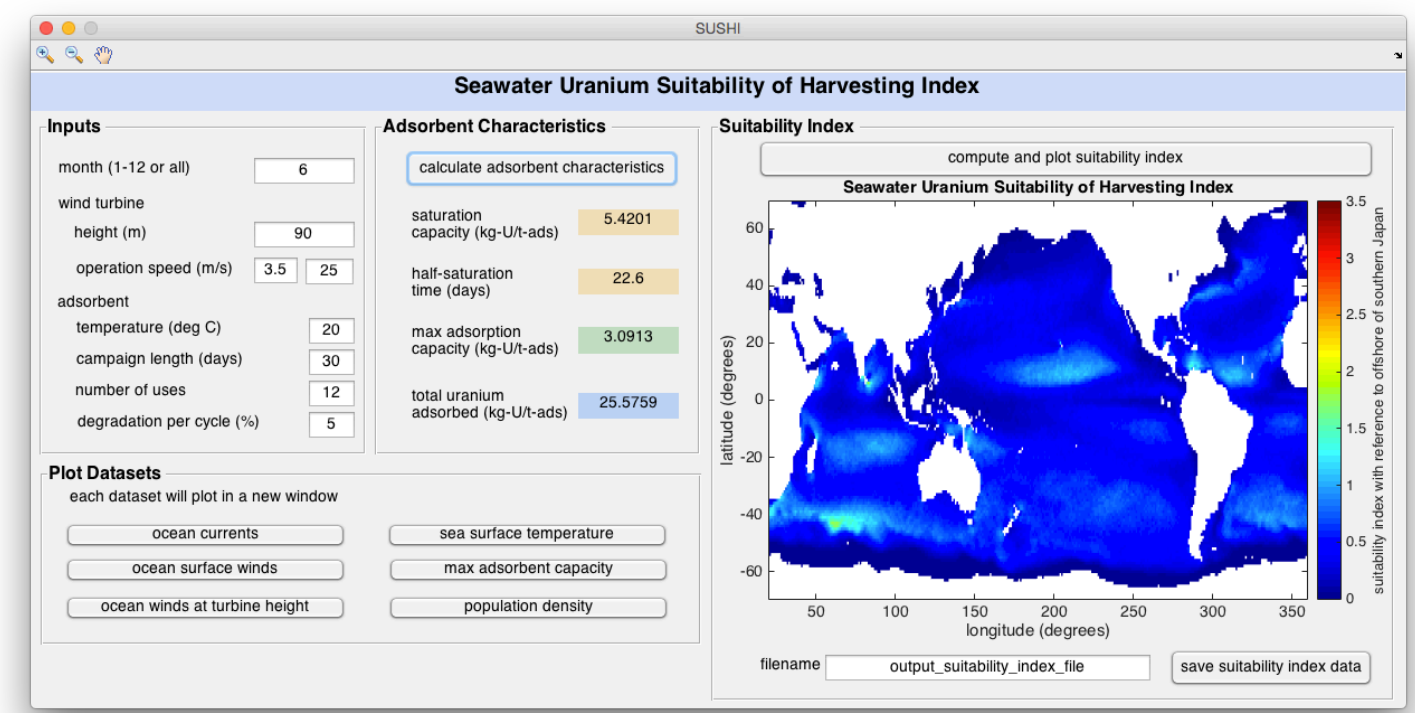

Figure 9-9: Screenshot of the GUI after selected datasets are plotted.

\subsection{Future Work}

At present, the Suitability Index only incorporates global wind and adsorbent capacity data as it relates to the feasibility of the colocation of uranium harvesters with floating offshore wind turbines. Future modifications to this system will include the competing effect on the adsorbent at increased temperature of biofouling reducing adsorbent uptake while temperature increasing it. This can be done be incorporated similarly as it was done in chapter 8 for the cost analysis of SMORE. The depth of the photic zone 
at regions around the world could also be incorporated to determine the likelihood of biofouling at locations across the globe. Or a simple factor of 30\% reduction in adsorbent uptake can be incorporated to account for biofouling.

Population density is currently not taken into account in this version of SUSHI, however could prove to be extremely important in the feasibility analysis of a symbiotic system to harvest uranium from seawater. For instance, while there are very strong winds in the middle of the ocean (which is very favorable for offshore wind development) the distance between these locations and any major population centers makes such deployments extremely costly and unreasonable. Hence, a factor to negatively weight regions based on their distance to population centers could remove these locations. This could also be incorporated by including ocean depth into SUSHI as ocean depth has shown to correlate with distance to shore.

Moreover, salinity is not considered in this model, although it has been shown to be a direct indicator of the amount of uranium present in the ocean (Owens et all, 2011). Incorporating global salinity data as well as any other global factors that affect the amount of uranium present in the ocean can greatly enhance the SUSHI tool to highlight regions with higher or exclude regions with lower uranium content.

Finally, the current SUSHI tool only considers the AF1 adsorbent. It should be expanded to include the possibility of all adsorbents developed by ORNL as described in chapter 2 (i.e. AF1, 38H, AI8). 


\section{Chapter 10}

\section{Conclusions and Future Work}

This thesis focused on the integration of a uranium harvesting system into an offshore wind turbine tower, taking in to account various properties and characteristics of the adsorbent polymer as well as the structure of the offshore wind turbine.

The characteristics of the adsorbent polymers that were referenced and utilized in the designs investigated in this thesis were presented in chapter 2 . The uranium uptake of the adsorbent has been shown to be sensitive to both temperature and biofouling. Furthermore, a model was developed by which the uranium uptake of the adsorbent can be optimized by tuning mechanical parameters of the system for a specific adsorbent.

Chapter 3 detailed some of the mechanical properties of the adsorbent, which was found to be inherently weak in tensile strength and durability. This chapter also presented a strategy in which the mechanical and chemical requirements of the uranium harvester may be decoupled by using a permeable, hard outer shell to protect an adsorbent fiber interior.

To determine if such shell enclosures would impede the uptake of uranium by the adsorbent, chapter $\mathbb{t}$ described the design and testing of various shell enclosures in a time series flume experiment. In the experiment, six shell enclosure designs were developed and tested in a recirculating flume. A control adsorbent with no shell enclosure was also included in the experiment. The results indicated that there was no significant effect on the uptake of uranium by the shell enclosures.

Chapter 5 built upon the promising results of the flume experiment to detail the design of a Symbiotic Machine for Ocean uRanium Extraction (SMORE), which utilizes shell enclosures that decouple the mechanical and chemical requirements of the system. The design incorporated the use of shell enclosures fabricated into a ball-chain net that can be powered to move the adsorbent net as desired. The design also utilized multiple subsystems to reduce the risk of all systems failing at once. A design tool was developed to allow a quick examination of the design space for a variety of input parameters such as turbine size, adsorbent type, ocean current speed, and stationary versus continuous motion of the ball-chain net.

To investigate whether a stationary or continuously moving ball-chain net would be beneficial for uranium uptake (the rationale behind the latter being that a continuously moving system would induce more water flow to the adsorbents), a 56-day ocean 
test of two 1/10th physical scale prototypes was conducted and described in chapter 6]. In collaboration with Woods Hole Oceanographic Institute, a novel method using radium adsorbing fibers was employed to determine water flow. The results indicated that the continuous system increases water flow to the adsorbents as hypothesized, while also reducing biofouling on the shells, which could result in a higher uranium uptake.

Based on the results of the ocean test, chapter $\square$ presented the testing of two 1/150th scale SMORE designs that utilized a continuously moving ball-chain net. The scale models were tested in the MIT Tow Tank to determine the resulting hydrodynamic response of the system, which was compared to that of an unmodified floating wind turbine. The tests showed that the addition of the uranium harvester to the floating offshore wind turbine did not have a significant effect on the hydrodynamic response of the system.

Chapter $\mathbf{8}$ detailed the cost-analysis of a full-scale SMORE design by utilizing a discounted cash flow technique to follow the costs of a unit mass of adsorbent accrued throughout its lifetime, as was done in previous cost analyses (Schneider and Sachde, 2013; Byers and Schneider, 2016a; Byers et al., 2016; Haji et al., 2017). The results showed the production cost of uranium from seawater using a SMORE deployment strategy ranged from $\$ 313-\$ 593 / \mathrm{kgU}$. Compared to the cost of seawater uranium using the reference deployment strategy (Tamada et al, 20106; Schneider and Linder, 2014), in the best case scenario using a SMORE deployment strategy reduced the cost of seawater uranium by $27 \%$ to $\$ 313 / \mathrm{kgU}$, within $4.3 \%$ of the peak uranium cost of $\$ 300 / \mathrm{kgU}$ in 2007.

A Seawater Uranium Suitability of Harvesting Index (SUSHI) was developed in chapter 9 to examine the locations and conditions where a SMORE device could be installed. A graphical user interface tool for quick analysis of worldwide locations was also described. The tool takes into account important properties such as ocean temperature, wind speed, and ocean current speed.

Although the production cost of seawater uranium from SMORE is still much greater than that of terrestrial uranium sources, the cost difference in this component of nuclear fuel may be affordable given the extremely large capital and operating costs of nuclear power plants. For instance, the levelized cost of an advanced light water reactor is approximately $\$ 66.65 / \mathrm{MWh}$. Of that, $\$ 6.63 / \mathrm{MWh}$ is due to the fuel cost. The fuel cost includes the price of natural uranium, the cost of its conversion to uranium hexaflouride, $\mathrm{UF}_{6}$, the price of enriching uranium hexaflouride, $\mathrm{UF}_{6}$, and the price of fabricating $\mathrm{UO}_{2}$ fuel from enriched $\mathrm{UF}_{6}$. The cost of natural uranium (including its conversion to uranium hexaflouride, $\mathrm{UF}_{6}$ ) accounts for approximately $42 \%$ of the total fuel cost. Therefore, the cost of the natural uranium alone is about $\$ 2.77 / \mathrm{MWh}$, or $4.16 \%$ of the total cost of an advanced light water reactor (Rothwell, 2016). Thus, if the cost of natural uranium were to double or triple, it would not greatly affect the overall capital and operating cost of a nuclear reactor.

Moreover, Rothwell (2016) cites that nuclear reactors which require reprocessed uranium for fuel, such as those that use mixed oxide (MOX) fuel, have a breakeven price of $\$ 210-\$ 560 / \mathrm{kgU}$. Hence, with a symbiotic device to harvest uranium from seawater, such as SMORE detailed in this thesis, the production price of seawater 
uranium becomes cost competitive with MOX technologies, which currently account for almost 5\% of new nuclear fuel (World-Nuclear.org, 2016).

Given that the specifics of offshore wind turbines are often proprietary and closely guarded secrets to each manufacturer, the design tools developed in this thesis were based on the 5-MW NREL OC3-Hywind, for which publicly available data exist (Jonkman et al, 2009; Jonkman, 2010). However, there are a wide variety of proposed offshore wind turbine systems for which such data is not easily available. For instance, the foundation of an offshore wind turbine differs greatly depending on the water depth, as shown in figure [0-1]. Additionally, the offshore wind turbine will have additional components such as ladder systems, platforms for maintenance operations, wiring, as well as access hatches along the turbine, all features that are often not disclosed to the public. The design tools developed in this thesis can easily be adapted for any new design or proposed support turbine. Furthermore, as was done in chapter 7, the dynamic analysis of the proposed system for a specific offshore wind turbine can be investigated both numerically and experimentally.

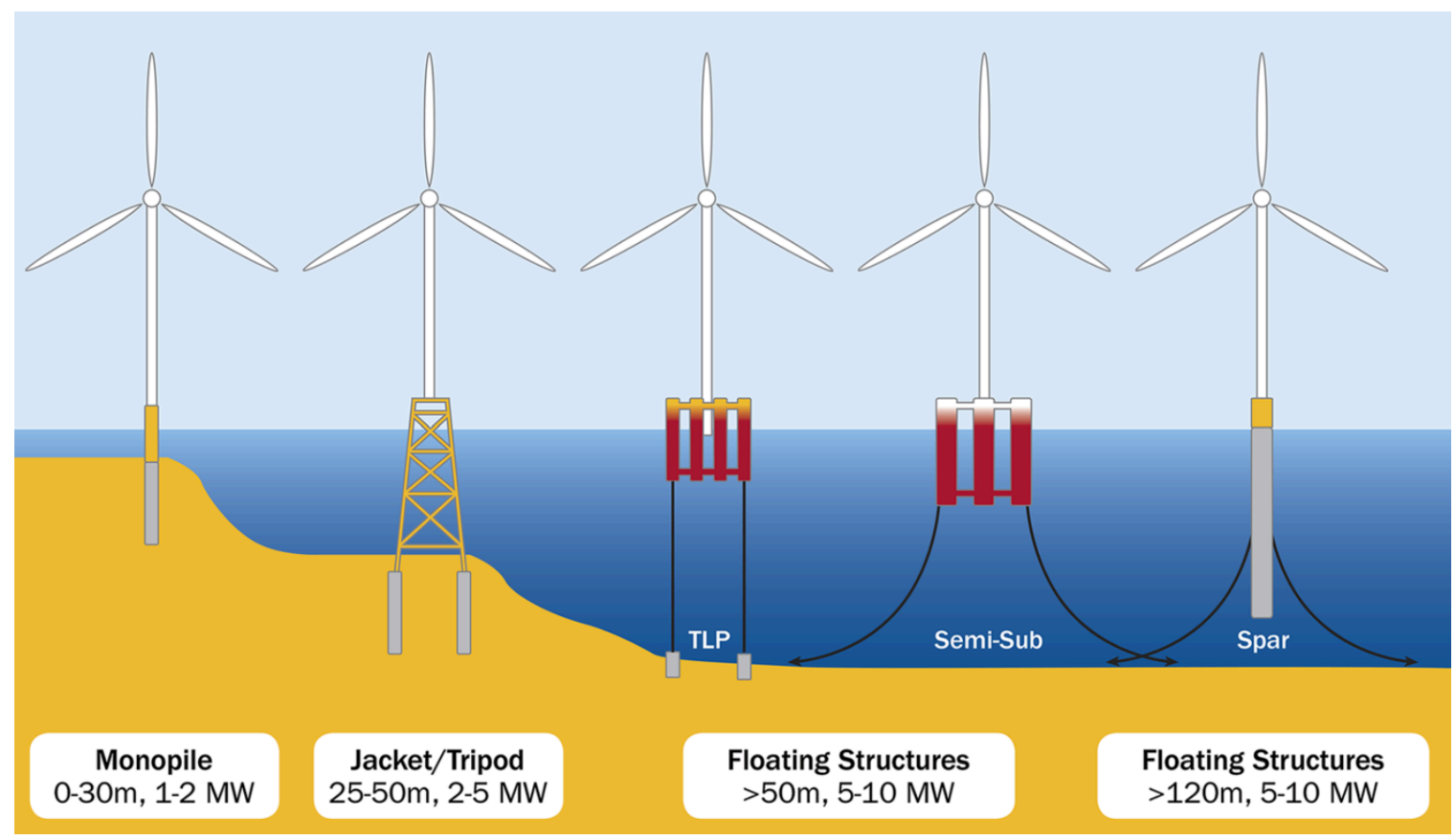

Figure 10-1: Types of offshore wind turbine foundations. Monopile and tripod/jacket foundations are currently proven technologies. Floating structures have been using three main types of foundations adapted from the oil and gas industry: the Tension Leg Platform (TLP), semi-submersible (Semi-sub), and Spar Buoy (Spar). This figure is reproduced from the European Wind Energy Association (2013).

In addition to the extraction of uranium from seawater, the adsorbents presented in this thesis could be used to extract other metals. For instance, the current fibers also extract vanadium, a prominent steel alloy, from seawater. Additionally, cobalt, a metal with applications in battery technology, is also extractable with this adsorbent as it exists in the ocean in large quantities at depths easily reached by combining 
a harvesting system to the bottom of an offshore wind turbine (Saito and Moffett, 2(1)(12).

One issue inherent to all current adsorbent technologies is the use of plastics such as polyethylene and polypropylene. With the need to place massive amounts of this plastic-based adsorbent in the ocean, all deployment strategies will likely be subject to Marine Pollution Convention (MARPOL), the international treaty that regulates the disposal of garbage aboard ships. According to Annex V of MARPOL, discharge of plastics is prohibited anywhere in the sea. In the United States, the Marine Plastic Pollution Research and Control Act passed in 1987 implements the provisions of MARPOL's Annex V, making it illegal to throw plastic into waters within the United States' Exclusive Economic Zone (a zone within 200 miles of the US coastline). These laws and restrictions could make extraction of metals from seawater using current adsorbent technologies difficult. Plastics, such as polyethylene, were originally incorporated into the adsorbents to increase tensile strength and durability. With the development of the shell enclosures in this thesis, the adsorbent no longer needs to have such high strengths, and therefore can be made out of a number of other materials. The investigation into alternative, non-plastic based adsorbents that also have higher adsorption capacity should be the next step in developing the technology for the commercialization of systems that extract metals from seawater. 


\section{Bibliography}

Cost estimating guidelines for Generation IV nuclear energy systems, Revision 4.2. The Economic Modeling Working Group Of the Generation IV International Forum, Generation IV International Forum, 2007.

S. Abarzua, S. Jakubowski, S. Eckert, and P. Fuchs. Biotechnological investigation for the prevention of marine biofouling II. Blue-green algae as potential producers of biogenic agents for the growth inhibition of microfouling organisms. Botanica Marina, 42(5):459-465, 1999.

T. S. Anirudhan, A. R. Tharun, S. Rijith, and P. S. Suchithra. Synthesis and characterization of a novel graft copolymer containing carboxyl groups and its application to extract uranium (VI) from aqueous media. Journal of Applied Polymer Science, 122(2):874-884, 2011.

Australian Bureau of Agricultural and Resource Economics and Sciences. Summary of australian for uranium: Worksheet 344-uranium prices. Available from Department of Agriculture, Fisheries and Forestry, GPO Box 1563, Canberra, ACT 2601.

K. M. Berntsson, P. R. Jonsson, M. Lejhall, and P. Gatenholm. Analysis of behavioural rejection of micro-textured surfaces and implications for recruitment by the barnacle balanus improvisus. Journal of Experimental Marine Biology and Ecology, 251:59-83, 2000.

K. Buesseler, 2017. Personal communication.

M. Byers. Optimization of the passive recovery of uranium from seawater. Master's thesis, The University of Texas at Austin, 2015.

M. F. Byers and E. Schneider. Uranium from Seawater Cost Analysis: Recent Updates. 2015.

M. F. Byers and E. Schneider. Optimization of the Passive Recovery of Uranium from Seawater. Industrial \&3 Engineering Chemistry Research, 55(15):43514361, 2016a.

M. F. Byers and E. Schneider. Uranium from Seawater Cost Analysis: Recent Updates. 2016b. 
M. F. Byers, M. N. Haji, A. H. Slocum, and E. Schneider. A Higher Fidelity Cost Analysis of Wind and Uranium from Seawater Acquisition symBiotic Infrastructure. 2016.

M. E. Callow and R. L. Fletcher. The influence of low surface energy materials on bioadhesion - a review. International Biodeterioration $\&$ Biodegradation, 34: 333-348, 1994.

M. E. Callow, A. R. Jennings, A. B. Brennan, C. E. Seegert, A. Gibson, L. Wilson, A. Feinberg, R. Baney, and J. A. Callow. Microtopographic cues for settlement of zoospores of the green fouling alga enteromorpha. Biofouling, 18:229-236, 2002.

S. K. Chakrabarti. Offshore Structure Modeling. World Scientific, Singapore, SGP, 1994.

L. D. Chambers, K. R. Stokesa, F. C. Walsha, and R. J. K. Wooda. Modern approaches to marine antifouling coatings. Surface and Coatings Technology, 201: 3642-3652, 2006.

G. R. Choppin. Soluble Rare Earth and Actinide Species in Seawater. Marine Chemistry, 28:19-26, 1989.

J. H. Conway and N. J. A. Sloane. Sphere Packings, Lattices, and Groups. SpringerVerlag, New York, 1993.

S. Das, A. K. Pandey, A. Athawale, V. Kumar, Y. K. Bhardwaj, S. Sabharwal, and V. K. Manchanda. Chemical aspects of uranium recovery from seawater by amidoximated electron-beam-grafted polypropylene membranes. Desalination, 232, 2008.

S. Das, A. K. Pandey, A. A. Athawale, and V. K. Manchanda. Exchanges of uranium(VI)-species in amidoxime functionalized sorbents. Journal of Physical Chemistry, 113, 2009.

S. Das, Y. Oyola, R. T. Mayes, C. J. Janke, L.-J. Kuo, G. Gill, J. R. Wood, and S. Dai. Extracting Uranium from Seawater: Promising AI Series Adsorbents. Industrial \& Engineering Chemistry Research, 55(15):4103-4109, 2016a.

S. Das, Y. Oyola, Richard T. Mayes, Chris J. Janke, L.-J. Kuo, G. Gill, J. R. Wood, and S. Dai. Extracting Uranium from Seawater: Promising AF Series Adsorbents. Industrial \& Engineering Chemistry Research, 55(15):4110-4117, 2016b.

R. V. Davies, J. Kennedy, R. W. McIroy, R. Spence, and K. M. Hill. Extraction of Uranium From Sea Water. Nature, 203(495):1110-1115, 1964.

L. V. R. de Messano, L. Sathler, L. Y. Reznik, and R. Coutinho. The effect of biofouling on localized corrosion of the stainless steels N08904 and UNS S32760. International Biodeterioration \& Biodegradation, 63:607-614, 2009. 
Mayyada M.H. El-Sayed, Heba A. Hani, and Mohamed H. Sorour. Polymeric ion exchangers for the recovery of ions from brine and seawater. Journal of Chemical Engineering \& Process Technology, 2:1020, 2014.

D. L. Elliot, C. G. Holladay, W. R. Barchet, H. P. Foote, and W. F. Sandusky. Wind Energy Resource Atlas of the United States. Solar Energy Research Institute, Golden, Colorado, 1987.

J. Emsley. Nature's Building Blocks: An A to Z Guide to the Elements. Oxford Univ. Press, 2001.

Energy Information Administration. International Energy Outlook 2013. Technical report, U.S. Department of Energy, July 2013.

European Wind Energy Association. Deep Water: The Next Step for Offshore Wind Energy. A report by the European Wind Energy Association, Brussels, Belgium, 2013.

H. Fravel. Understanding the critical relationship between reverse osmosis recovery rates and concentration factors, 2014. https://www.wateronline.com/doc/ understanding-the-critical-relationship-between-reverse-osmosisrecovery-rates-and-concentration-tactors-0001, Accessed: 2017-04-23.

P. Gerland, A. E. Raftery, H. Ševčková, N. Li, D. Gu, T. Spoorenberg, L. Alkema, B. K. Fosdick, J. Chunn, N. Lalic, G. Bay, T. Buettner, G. K. Heilig, and J. Wilmoth. World population stabilization unlikely this century. Science, 2014.

G. A. Gill, L.-J. Kuo, J. R. Wood, and C. Janke. Complete Laboratory Evaluation and Issue a Report on the Impact of Temperature on Uranium Adsorption. Department of Energy Milestone report, (September), 2014.

G. A. Gill, L.-J. Kuo, J. Strivens, J. Wood, N. J. Schlafer, C. Tsouris, A. Ladshaw, and S. Yiacoumi. Investigations into the Effect of Current Velocity on AmidoximeBased Polymeric Uranium Adsorbent Performance. Department of Energy Milestone report, (December), 2015.

G. A. Gill, L.-J. Kuo, C. J. Janke, J. Park, R. T. Jeters, G. T. Bonheyo, H-B Pan, C. Wai, T. Khangaonkar, L. Bianucci, J. R. Wood, M. G. Warner, S. Peterson, D. G. Abrecht, R. T. Mayes, C. C. Tsouris, Y. Oyola, J. E. Strivens, N. J. Schlafer, R. S. Addleman, W. Chouyyok, S. Das, J. Kim, K. Buesseler, C. Breier, and E. D'Alessandro. The Uranium from Seawater Program at the Pacific Northwest National Laboratory: Overview of Marine Testing, Adsorbent Characterization, Adsorbent Durability, Adsorbent Toxicity, and Deployment Studies. Industrial \&6 Engineering Chemistry Research, (15), 2016.

$\mathrm{H}$ and $\mathrm{H}$ Tooling. Basic Tube Bending Guide. 
M. N. Haji, C. Delmy, J. Gonzalez, and A. H. Slocum. Uranium extraction from seawater using adsorbent shell enclosures via a symbiotic offshore wind turbine device. Proceedings of tje 26th International Ocean and Polar Engineering Conference, 2016.

M. N. Haji, M. Byers, E. Schneider, and A. H. Slocum. Cost Analysis of Wind and Uranium from Seawater Acquisition symBiotic Infrastructure using Shell Enclosures. Proceedings of the 2015 American Nuclear Society Winter Meeting and Nuclear Technology Expo, 2017.

A. M Hamlet. Uranium extraction from seawater: Investigating the hydrodynamic behavior and performance of porous shells. Master's thesis, Massachusetts Institute of Technology, 2017.

J. M. Hills and J. C. Thomason. The effects of scales of surface roughness on the settlement of the barnacle (Semibalanus balanoides). Biofouling, 12:57-69, 1998.

D. Howell and B. Behrends. A methodology for evaluating biocide release rate, surface roughness and leach layer formation in a TBT-free, self-polishing antifouling coating. Biofouling, 22:303-315, 2006.

J. Hu, H. Ma, Z. Xing, X. Liu, L. Xu, R. Li, C. Ling, M. Wang, J. Li, and G. Wu. Preparation of Amidoximated UHMWPE Fiber by Radiation Grafting and Uranium Adsorption Test. Industrial \&6 Engineering Chemistry Research, (15), 2016.

Intergovernmental Panel on Climate Change. Climate Change 2007: Synthesis Report. Contribution of Working Groups I, II and III to the Fourth Assessment. World Meteorological Organization and the United Nations Environment Programme, 2007.

C. Janke, S. Das, and R. Mayes. Preparation of most promising braided and/or textile-based adsorbents for seawater testing. Technical report, U.S. Department of Energy, February 2014.

J. Jonkman. Definition of the Floating System for Phase IV of OC3. Technical report, U.S. Department of Energy, May 2010.

J. M. Jonkman, S. Butterfield, W. Musial, and G. Scott. Definition of a 5-MW reference wind turbine for offshore system development. Technical report, U.S. Department of Energy, February 2009.

M. Kanno. Present status of study on extraction of uranium from sea water. Journal of Nuclear Science and Technology, 21(1):1-9, 1984.

S. P. Kelley, P. S. Barber, P. H. Mullins, and R. D. Rogers. Structural clues to $\mathrm{UO}_{2}{ }^{2+} / \mathrm{VO}_{2}{ }^{+}$competition in seawater extraction using amidoxime-based extractants. Chemical Communications, 50:12504-12507, 2014. 
J. Kim, Y. Oyola, C. Tsouris, C. R. Hexel, R. T. Mayes, C. J. Janke, and S. Dai. Characterization of uranium uptake kinetics from seawater in batch and flow-through experiments. Industrial \&5 Engineering Chemistry Research, 52:9433-9440, 2013a.

J. Kim, C. Tsouris, R. T. Mayes, Y. Oyola, T. Saito, C. J. Janke, S. Dai, E. Schneider, and D. Sachde. Recovery of Uranium from Seawater: A Review of Current Status and Future Research Needs. Separation Science and Technology, 48:367-387, 2013b.

J. Kim, C. Tsouris, Y. Oyola, C. J. Janke, R. T. Mayes, S. Dai, G. Gill, L.-J Kup, J. Wood, K.-Y. Choe, E. Schneider, and H. Lindner. Uptake of uranium from seawater by amidoxime-based polymeric adsorbent: Field experiments, modeling, and updated economic assessment. Industrial \& Engineering Chemistry Research, 53:6076-6083, 2014.

J. Kluger. Synergistic Design of a Combined Floating Wind Turbine - Wave Energy Converter. PhD thesis, Massachusetts Institute of Technology, 2017.

L.-J. Kuo, G. Gill, H.-B. Pan, and C. Wai. Improving Adsorbent Reusability for Extraction of Uranium from Seawater. 2015.

L.-J. Kuo, C. J. Janke, J. R. Wood, J. E. Strivens, S. Das, Y. Oyola, R. T. Mayes, and G. A. Gill. Characterization and Testing of Amidoxime-Based Adsorbent Materials to Extract Uranium from Natural Seawater. Industrial $\& 3$ Engineering Chemistry Research, 55(15):4285-4293, 2016.

A. Ladshaw, L.-J. Kuo, J. Strivens, J. Wood, N. Schlafer, S. Yiacoumi, C. Tsouris, and G. Gill. Influence of Current Velocity on Uranium Adsorption from Seawater Using an Amidoxime-Based Polymer Fiber Adsorbent. Industrial $\&$ Engineering Chemistry Research, 56:2205-2211, 2017.

A. Lakretz, E. Z. Ron, and H. Mamane. Biofouling control in water by various uvc wavelengths and doses. Biofouling, 26:257-267, 2010.

C. H. Lee and J. N. Newman. WAMIT@ User Manual. WAMIT, Inc., Chestnut Hill, MA, 2006.

M. Lejars, A. Margaillan, and C. Bressy. Fouling release coatings: A nontoxic alternative to biocidal antifouling coatings. Chemical Reviews, 112(8):4347-4390, 2012.

A. Mickey. Uranium Has Bottomed: Two Uranium Bulls to Jump on Now. UraniumSeek.com, 2008. Published: 2008-08-22.

W. S. Moore. Sampling ${ }^{228} \mathrm{Ra}$ in the deep ocean. Deep Sea Research and Oceanographic Abstracts, 23:647-651, 1976.

W. S. Moore. Radium isotope measurements using germanium detectors. Nuclear Instruments and Methods in Physics Research, 223:407-411, 1984. 
W. S. Moore, H. Astwood, and C. Lindstrom. Radium isotopes in coastal waters on the Amazon shelf. Geochimica et Cosmochimica Acta, 59:4285-4298, 1995.

A. Myhr, C. Bjerkseter, A. Ågotnes, and T. A. Nygaard. Levelised cost of energy for offshore floating wind turbines in a life cycle perspective. Renewable Energy, 66: 714-728, 2014.

J. Newman and P. Sclavounos. The computation of wave loads on large offshore structures. In Proceedings of BOSS, 1988.

C. Not, K. Brown, B. Ghaleb, and C. Hillaire-Marcel. Conservative behavior of uranium vs. salinity in Arctic sea ice and brine. Mar. Chem., 130-131:33-39, 2012.

OECD Nuclear Energy Agency. Managing Environmental and Health Impacts of Uranium Mining. Technical report, OECD Nuclear Energy Agency and the International Atomic Energy Agency, 2014.

OECD Nuclear Energy Agency. Uranium 2016: Resources, Production and Demand. Technical report, OECD Nuclear Energy Agency and the International Atomic Energy Agency, 2016.

H. Omichi, A. Katakai, T. Sugo, and J. Okamoto. A New Type of Amidoxime-GroupContaining Adsorbent for the Recovery of Uranium from Seawater. III. Recycle use of adsorbent. Separation Science and Technology, 21:563-574, 1986.

S. A. Owens, K. O. Buesseler, and K. W. W. Sims. Re-evaluating the ${ }^{238} \mathrm{U}$-salinity relationship in seawater: Implications for the ${ }^{238} \mathrm{U}-\mathrm{Th}-234$ disequilibrium method. Marine Chemistry, 127(1-4):31-39, 2011.

H.-B. Pan, W. Liao, C. M. Wai, Oyola Y., C. J. Janke, G. Tian, and Rao L. Carbonate- $\mathrm{H}_{2} \mathrm{O}_{2}$ leaching for sequestering uranium from seawater. Dalton transactions, 43(28):10713-8, 2014.

H.-B. Pan, L.-J. Kuo, J. Wood, J. E. Strivens, G. A. Gill, C. J. Janke, and C. M. Wai. Towards understanding $\mathrm{KOH}$ conditioning of amidoxime-based polymer adsorbents for sequestering uranium from seawater. RSC Advances, 5(122), 2015.

H.-B. Pan, C. M. Wai, L.-J. Kuo, G. Gill, G. Tian, L. Rao, S. Das, R. T. Mayes, and C. J. Janke. Bicarbonate Elution of Uranium from Amidoxime-Based Polymer Adsorbents for Sequestering Uranium from Seawater. Chemistry Select, 2(13): 3769-3774, 2017.

C. Park. Contemporary Engineering Economics. pages 220-227, 2016.

J. Park, G. A. Gill, J. E. Strivens, L.-J. Kuo, R. Jeters, A. Avila, J. Wood, N. J. Schlafer, C. J. Janke, E. A. Miller, M. Thomas, R. S. Addleman, and G. Bonheyo. Effect Of Biofouling On The Performance Of Amidoxime-Based Polymeric Uranium Adsorbents. Industrial \&6 Engineering Chemistry Research, (15), 2016. 
PelaFlow Consulting. Wind turbine power output variation with steady wind speed, 2008. http://www.wind-power-program.com/turbine_characteristics.htm, Accessed: 2014-10-12.

M. Picard, C. Baelden, Y. Wu, L. Chang, and A. H. Slocum. Extraction of Uranium from Seawater: Design and Testing of a Symbiotic System. Nuclear Technology, 188(2), 2014.

A. I. Railkin. Marine Biofouling: Colonization Processes and Defenses. CRC Press, 2003.

R. W. Reynolds, N. A. Rayner, T. M. Smith, D. C. Stokes, and W. Wang. An improved in situ and satellite SST analysis for climate. Journal of Climate, 15: 1609-1625, 2002.

G. Rothwell. Economics of Nuclear Power. Routledge, 2016.

D. J. Sachde. Uranium Extraction from Seawater: An Assessment of Cost, Uncertainty and Policy Implications. Master's thesis, The University of Texas at Austin, 2011.

M. A. Saito and J. W. Moffett. Temporal and spatial variability of cobalt in the Atlantic Ocean. Geochemica et Cosmochimica Acta, 66:1943-1953, 2002.

T. Saito, S. Brown, S. Chatterjee, J. Kim, C. Tsouris, R. Mayes, L.-J. Kuo, Y. Gill, G. Oyola, C. Janke, and S. Dai. Uranium recovery from seawater: development of fiber adsorbents prepared via atom-transfer radical polymerization. Journal of Materials Chemistry A, 2:14674-14681, 2014.

J. P. J. Scanlan. Journal of Inorganic and Nuclear Chemistry, 39, 1977.

H. J. Schenk, L. Astheimer, E. G. Witte, and K. Schwochau. Development of Sorbers for the Recovery of Uranium from Seawater. 1. Assessment of Key Parameters and Screening Studies of Sorber Materials. Separation Science and Technology, 17, 1982.

E. Schneider and H. Linder. Updates to the Estimated Cost of Uranium Recovery from Seawater. In Proceedings of the 19th Pacific Basin Nuclear Conference, 2014.

E. Schneider and D. Sachde. The Cost of Recovering Uranium from Seawater by a Braided Polymer Adsorbent System. Sci. Glob. Sec., 21(2):134-163, 2013.

M. Schwartz, D. Heimiller, S. Haymes, and W. Musial. Assessment of Offshore Wind Energy Resources for the United States Assessment of Offshore Wind Energy Resources for the United States. Technical report, National Renewable Energy Laboratory, 2010.

K. Sekiguchi, K. Saito, S. Konishi, S. Furusaki, T. Sugo, and H. Nobukawa. Effect of seawater temperature on uranium recovery from seawater using amidoxime adsorbents. Industrial \&6 Engineering Chemistry Research, 33(3):662-666, 1994. 
N. Seko, A. Katakai, S. Hasegawa, M. Tamada, N. Kasai, H. Takeda, T. Sugo, and K. Saito. Aquaculture of uranium in seawater by a fabric-adsorbent submerged system. Nuclear Technology, 144(2):274-278, 2003.

N. Seko, A. Katakai, M. Tamada, and S. Takanobu. Fine Fibrous Amidoxime Adsorbent Synthesized by Grafting and Uranium AdsorptionElution Cyclic Test with Seawater. Separation Science and Technology, 39(16):3753-3767, 2004.

Alexander H. Slocum. FUNdaMENTALS of Design. 2008.

C. P. Sparks. Fundamentals of Marine Riser Mechanics - Basic Principles and Simplified Analysis. PennWell, 2007.

T. Sugo, M. Tamada, T. Seguchi, T. Shimizu, M. Uotani, and R. Kashima. Recovery System for Uranium from Seawater with Fibrous Adsorbent and Its Preliminary Cost Estimation. Journal of the Atomic Energy Society of Japan, 43(10):1010-1016, 2001.

T. Sugo H. Ogura T. Suzuki, K. Saito and K. Oguma. Fractional Elution and Determination of Uranium and Vanadium Adsorbed on Amidoxime Fiber from Seawater. Analytical Sciences.

M. Tamada. Current status of technology for collection of uranium from seawater. Japan Atomic Energy Agency, 2009.

M. Tamada, N. Seko, N. Kasai, , and T. Shimizu. Cost estimation of uranium recovery from seawater with system of braid type adsorbent. Transactions of the Atomic Energy Society of Japan, 5(4):358-363, 2006.

C. Tien. Adsorption Calculations and Modeling. Butterworth- Heinemann, Newton, MA, 1994.

G. A. Tularam and M. Ilahee. Environmental concerns of desalinating seawater using reverse osmosis. Journal of Environmental Monitoring, 9(8):805-813, 2007.

Union of Concerned Scientists. Fact Sheet - Nuclear Power and Water: Quick Facts on Nuclear Power Generation and Water Use. Technical report, 2011. URL http://www.ucsusa.org/sites/default/files/legacy/assets/ documents/nuclear_power/fact-sheet-water-use.pdf.

University of Idaho. Uranium Recovery from Seawater: A Nation-Wide Consortium for Sustainable Energy - University of Idaho. http://uraniumfromseawater. engr.utexas.edu/partners/university-idaho, Accessed: 2017-04-15.

US Department of Energy. Statistical data of the uranium industry, 1981. GJO100(81). Available online at WWW.osti.gov/scitech/servlets/purl/6137459.

Ux Consulting Company, LLC. Uxc nuclear fuel price indicators, 2015. Available online at WWw.uxc.com/review/uxc_Prices.aspx. 
R. van Reis and A. Zydney. Bioprocess membrane technology. Journal of Membrane Science, 297(1):16-50, 2007.

S. Vukovic, L. A. Watson, S. O. Kang, R. Custelcean, and B. P. Hay. How amidoximate binds the uranyl cation. Inorganic Chemistry, 51(6):3855-3859, 2012.

D. R. Walt, J. B. Smulow, S. S. Turesky, and R. G. Hill. The effect of gravity on initial microbial adhesion. Journal of Colloid and Interface Science, 107:334-336, 1985.

C.-Z. Wang, J.-H. Lan, Q.-Y. Wu, Q. Luo, Y.-L. Zhao, X.-K. Wang, Z.-F. Chai, and W.-Q. Shi. Theoretical Insights on the Interaction of Uranium with Amidoxime and Carboxyl Groups. Inorganic Chemistry, 53:9466-9476, 2014.

P. A. White, J. Kalff, J. B. Rasmussen, and J. M. Gasol. The effect of temperature and algal biomass on bacterial production and specific growth rate in freshwater and marine habitats. Microbial Ecologycology, 21:99-118, 1991.

World-Nuclear.org. Mixed oxide (mox) fuel, 2016. http://www.world-nuclear. org/information-library/nuclear-fuel-cycle/fuel-recycling/ mixed-oxide-fuel-mox.aspx, Accessed: 2017-04-23.

Z. Xing, J. Hu, M. Wang, W. Zhang, S. Li, Q. Gao, and G. Wu. Properties and evaluation of amidoxime-based UHMWPE fibrous adsorbent for extraction of uranium from seawater. Science China Chemistry, 56(11):1504-1509, 2013.

A. Yamanaka, Y. Izumi, T. Kitagawa, T. Terada, H. Hirahata, K. Ema, H. Fujishiro, and S. Nishijima. The effect of gamma-irradiation on thermal strain of high strength polyethylene fiber at low temperature. Journal of Applied Polymer Science, 102: 204-209, 2006.

A. Zhang, T. Asakura, and G. Uchiyama. The adsorption mechanism of uranium (VI) from seawater on a macroporous fibrous polymeric adsorbent containing amidoxime chelating functional group. Reactive and Functional Polymers, 57(1):6776, 2003.

Y. N. Zhao, M. H. Wang, Z. F. Tang, and G. Z. Wu. Effect of gamma-ray irradiation on the structure and mechanical properties of UHMWPE fibers. Polymer Materials Science \&3 Engineering, 26:32-35, 2010. 